\title{
Catalytic diastereo- and enantioselective synthesis of 2-imidazolinones
}

Pablo Martínez-Pardo, Gonzalo Blay, *Alba Escrivá-Palomo, Amparo Sanz-Marco, Carlos Vila and José R. Pedro*

Departament de Química Orgànica- Facultat de Química, Universitat de València, C/ Dr. Moliner 50, 46100-Burjassot, Spain.

\section{Table of Contents}

Optimization of the reaction conditions. Further experiments.

Table S1. Effect of molar ratio SQ3/ silver salt $\mathrm{S} 2$

Experimental Procedures S3

Synthesis and characterization data for SQ4 S3-S4

Synthesis and characterization data for nitrones $\mathbf{1}$

Synthesis and characterization data for compounds $3-\mathbf{8}$ S10-S24

Synthesis of compound 3aa at $1 \mathrm{mmol}$ scale S24-S25

References S25

NMR spectra and HPLC traces for compounds 3-8 


\section{Results and Discussion}

Optimization of the reaction conditions. Further experiments.

Table S1. Reaction of nitrone 1a and tert-butyl isocyanoacetate (2a) catalyzed by squaramides SQ3. Effect of molar ratio SQ3/ silver salt. $^{\mathrm{a}}$

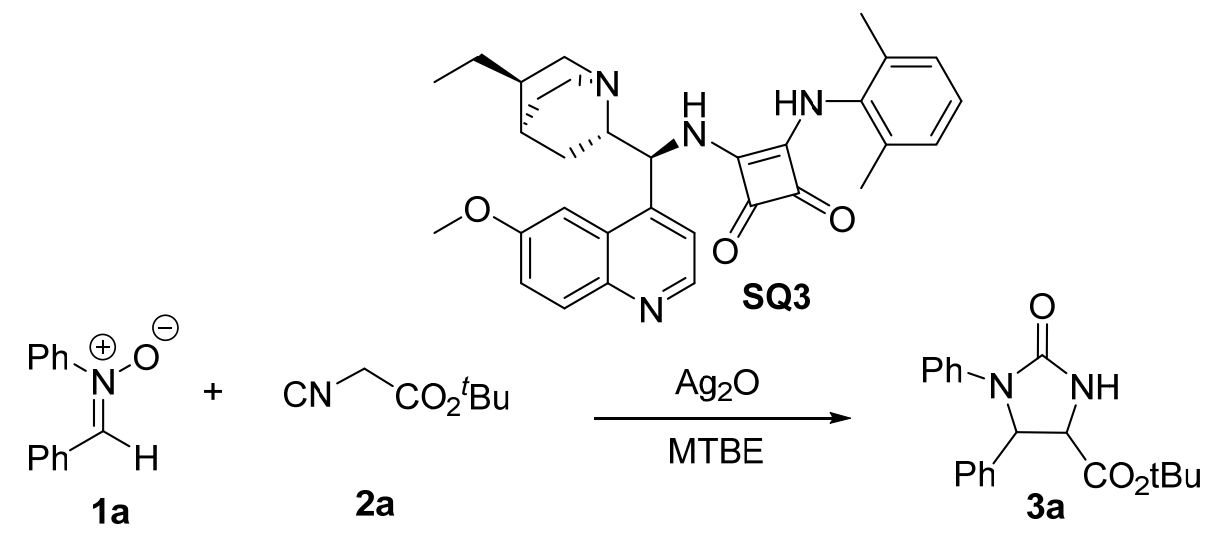

\begin{tabular}{|c|c|c|c|c|c|c|}
\hline Entry & SQ3 (mol \%) & $\mathrm{Ag}_{2} \mathrm{O}(\mathrm{mol} \%)$ & $t(\mathrm{~d})$ & yield $(\%)^{b}$ & trans:cis ${ }^{\mathrm{c}}$ & ee $e_{\text {trans }}(\%)^{\mathrm{d}}$ \\
\hline 1 & $10 \%$ & $5 \%$ & 2 & 46 & $73: 27$ & 99 \\
\hline 2 & $10 \%$ & $10 \%$ & 4 & 36 & $74: 26$ & 91 \\
\hline 3 & $20 \%$ & $10 \%$ & 4 & 46 & $65: 35$ & 91 \\
\hline 4 & $5 \%$ & $10 \%$ & 2 & 62 & $67: 33$ & 86 \\
\hline 5 & $10 \%$ & $5 \%{ }^{\mathrm{e}}$ & 2 & 47 & $66: 34$ & 86 \\
\hline 6 & $10 \%$ & $5 \%{ }^{\mathrm{f}}$ & 2 & n.r. & & \\
\hline 7 & $10 \%$ & $5 \% \mathrm{~g}$ & 2 & n.r. & & \\
\hline
\end{tabular}

a Conditions: 1a $(0.13 \mathrm{mmol}), 2 \mathrm{a}(0,17 \mathrm{mmol}), \mathbf{S Q}, \mathrm{Ag}_{2} \mathrm{O}$, dioxane $(2 \mathrm{~mL})$, room temperature. ${ }^{b}$ Isolated yield after column chromatography. ${ }^{c}$ Determined by ${ }_{1} \mathrm{H}$ NMR. ${ }^{\mathrm{d}}$ Determined by HPLC over chiral stationary phases. ${ }^{\text {e }} \mathrm{Ag}_{2} \mathrm{SO}_{4}$ was used instead of $\mathrm{Ag}_{2} \mathrm{O}$ at $0{ }^{\circ} \mathrm{C}$. ${ }^{\mathrm{f}} \mathrm{CF}_{3} \mathrm{CO}_{2} \mathrm{Ag}$ was used instead of $\mathrm{Ag}_{2} \mathrm{O}$ 


\section{Experimental Procedures}

The enantioselective reactions were carried out in round bottom flasks closed with stoppers. All starting materials were obtained from commercial sources. Reactions were monitored by TLC analysis using Merck Silica Gel 60 F-254 thin layer plates. Flash column chromatography was performed on Merck silica gel 60, 0.040-0.063 mm. Melting points were determined in capillary tubes. NMR spectra were run at $300 \mathrm{MHz}$ for ${ }^{1} \mathrm{H}$ and at $75 \mathrm{MHz}$ for ${ }^{13} \mathrm{C}$ NMR using residual nondeuterated solvent $\left(\mathrm{CHCl}_{3}\right)$ as internal standard $(\delta 7.26$ and $77.0 \mathrm{ppm}$, respectively). Chemical shifts are given in ppm. The carbon type was determined by DEPT experiments. High resolution mass spectra (ESI) were recorded on a Q-TOF spectrometer equipped with an electrospray source with a capillary voltage of $3.3 \mathrm{kV}$ (ESI). Specific optical rotations were measured using sodium light (D line $589 \mathrm{~nm}$ ). Chiral HPLC analyses were performed in a chromatograph equipped with a UV diode-array detector using chiral stationary phase columns from Daicel or Phenomenex.

\section{Synthesis and characterization data for squaramide SQ4}

\section{3-((2,6-Diisopropylphenyl)amino)-4-methoxycyclobut-3-ene-1,2-dione ${ }^{1}$}

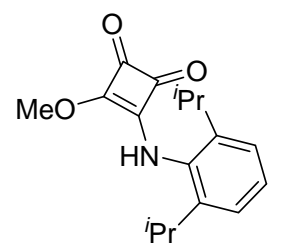

The title compound was prepared following a modified procedure: 2 2,6diisopropylaniline $(796 \mu \mathrm{L}, 4.2 \mathrm{mmol})$ was added to a solution of methyl squarate $(600 \mathrm{mg}, 4.2 \mathrm{mmol})$ and zinc trifluoromethanesulfonate $(305 \mathrm{mg}, 0.84$

mmol) in dichloromethane $(12 \mathrm{~mL})$. The reaction was stirred at room temperature for $48 \mathrm{~h}$, concentrated under reduced pressure and purified by chromatography column (EtOAc:hexane, 2:8) to obtain $552.9 \mathrm{mg}(46 \%)$ of the known semisquaramide. ${ }^{1}{ }^{1} \mathrm{H}$ NMR (300 $\left.\mathrm{MHz}, \mathrm{CDCl}_{3}\right) \delta 7.81(1 \mathrm{H}, \mathrm{s}, \mathrm{NH}), 7.40-7.30(1 \mathrm{H}, \mathrm{m}, \mathrm{Ar}), 7.18(2 \mathrm{H}, \mathrm{d}, J=7.7 \mathrm{~Hz}, \mathrm{Ar}), 4.18(3 \mathrm{H}, \mathrm{s}$, $\left.\mathrm{CH}_{3}\right), 3.07(2 \mathrm{H}$, hept, $J=6.9 \mathrm{~Hz}, \mathrm{CH}), 1.19\left(12 \mathrm{H}, \mathrm{d}, J=6.9 \mathrm{~Hz}, \mathrm{CH}_{3}\right) ;{ }^{13} \mathrm{C} \mathrm{NMR}\left(75 \mathrm{MHz}, \mathrm{CDCl}_{3}\right)$ $\delta 189.4(\mathrm{C}), 184.1(\mathrm{C}), 179.2(\mathrm{C}), 172.1(\mathrm{C}), 146.1(\mathrm{C}), 131.0(\mathrm{C}), 129.3(\mathrm{CH}), 123.6(\mathrm{CH}), 60.4$ $\left(\mathrm{CH}_{3}\right), 28.6(\mathrm{CH}), 23.5\left(\mathrm{CH}_{3}\right)$.

\section{3-((2,6-Diisopropylphenyl)amino)-4-(((S)-((1S,2S,4S,5R)-5-ethylquinuclidin-2-yl)(6-} methoxyquinolin-4-yl)methyl)amino)cyclobut-3-ene-1,2-dione (SQ 4)

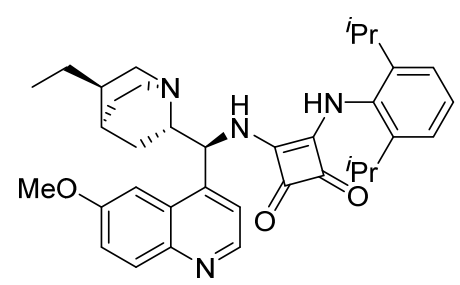

A mixture of the above squaramide $(194 \mathrm{mg}, 0.79 \mathrm{mmol})$ and $(1 S, 2 S, 4 S, 5 R)$-5-ethylquinuclidin-2-yl)(6-methoxyquinolin-4-yl)methanamine $^{3}(258 \mathrm{mg}, 0.79 \mathrm{mmol})$ in methanol $(5 \mathrm{~mL})$ was heated at reflux temperature for $48 \mathrm{~h}$. Then, the solvent was removed under reduced pressure and the mixture was purified by column chromatography eluting with 
EtOAc:MeOH (9:1) to give $339.6 \mathrm{mg}$ (58\%) of SQ 4. Slightly yellow solid, m.p. 188-2192 ${ }^{\circ} \mathrm{C}$; $[\alpha]_{\mathrm{D}^{25}}-51.8\left(c{ }^{2} .0, \mathrm{CHCl}_{3}\right){ }^{1} \mathbf{H}$ NMR $\left(500 \mathrm{MHz}, \mathrm{CDCl}_{3}\right) \delta 8.65(1 \mathrm{H}, \mathrm{d}, J=4.6 \mathrm{~Hz}, \mathrm{Ar}), 8.55(1 \mathrm{H}$, bs, NH), $7.94(1 \mathrm{H}, \mathrm{d}, J=7.7 \mathrm{~Hz}, \mathrm{Ar}), 7.60(1 \mathrm{H}, \mathrm{bs}, \mathrm{Ar}), 7.48-7.29(2 \mathrm{H}, \mathrm{m}, \mathrm{Ar}), 7.23(1 \mathrm{H}, \mathrm{d}, J=$ $6.3 \mathrm{~Hz}, \mathrm{Ar}), 7.09(1 \mathrm{H}, \mathrm{d}, J=7.2 \mathrm{~Hz}, \mathrm{Ar}), 6.78(1 \mathrm{H}, \mathrm{bs}, \mathrm{Ar}), 5.71(1 \mathrm{H}, \mathrm{bs}, \mathrm{NH}), 4.57(1 \mathrm{H}, \mathrm{bs}, \mathrm{CH})$, $3.95\left(3 \mathrm{H}, \mathrm{s}, \mathrm{CH}_{3}\right), 3.21(1 \mathrm{H}, \mathrm{bs}), 3.11-2.82(3 \mathrm{H}, \mathrm{m}), 2.56-2.40(2 \mathrm{H}, \mathrm{m}), 2.10(1 \mathrm{H}, \mathrm{d}, J=12.2 \mathrm{~Hz})$, 1.59-1.31 (4H, m), $1.22(8 \mathrm{H}, \mathrm{m}), 1.01(4 \mathrm{H}, \mathrm{d}, J=6.8 \mathrm{~Hz}), 0.84-0.65(6 \mathrm{H}, \mathrm{m}) ;{ }^{13} \mathrm{C}$ NMR $(125 \mathrm{MHz}$, $\left.\mathrm{CDCl}_{3}\right) \delta 183.6(\mathrm{C}), 182.3(\mathrm{C}), 168.9(\mathrm{C}), 166.5(\mathrm{C}), 158.4(\mathrm{C}), 147.47(\mathrm{CH}), 147.45(\mathrm{CH}), 147.3$ (C), $147.1(\mathrm{CH}), 144.8(\mathrm{CH}), 144.3(\mathrm{C}), 131.5(\mathrm{CH}), 129.4(\mathrm{CH}), 128.7(\mathrm{C}), 128.6(\mathrm{C}), 123.7$ $(\mathrm{CH}), 123.4(\mathrm{CH}), 122.6(\mathrm{CH}), 117.6(\mathrm{C}), 101.2(\mathrm{CH})$, 61.2, 57.8, 56.0, 53.1, 40.73, 37.4, 28.6, 28.4, 27.6, 25.8, 25.1, 24.1, 22.9, 22.1, 12.0. HRMS (ESI) $\mathrm{m} / \mathrm{z}: 581.3477[\mathrm{M}+\mathrm{H}]^{+}, \mathrm{C}_{36} \mathrm{H}_{45} \mathrm{~N}_{4} \mathrm{O}_{3}{ }^{+}$ requires 581.3486 .

\section{Synthesis and characterization data for nitrones $\mathbf{1}^{4}$}

General procedure A: The corresponding benzaldehyde $(1 \mathrm{mmol})$ was added to a solution of $N$ phenylhydroxylamine $(1 \mathrm{mmol})$ in ethanol $(1 \mathrm{~mL})$, followed of two drops of concentrated $\mathrm{HCl}$. The mixture was stirred at room temperature until it was completed (TLC). The nitrone was isolated by filtration under reduced pressure and washed with cold ethanol. In the cases where the nitrone does not precipitate, the solvent was removed under reduced pressure and the crude product purified by column chromatography eluting with hexane:EtOAc mixtures.

General procedure B: A mixture of $N$-phenylhydroxylamine $(1 \mathrm{mmol})$ and the corresponding benzaldehyde $(1 \mathrm{mmol})$ in ethanol $(4 \mathrm{~mL})$ was heated at reflux temperature for 2 hours until the reaction was completed (TLC). The solvent was removed under reduced pressure and the crude product was crystallized from hexane:EtOAc mixtures.

\section{$N$-1-Diphenylmethanimine oxide (1a) ${ }^{4}$}

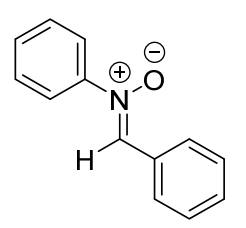

Procedure A. From $N$-phenylhydroxylamine $(300 \mathrm{mg}, 2.75 \mathrm{mmol})$ and benzaldehyde ( $278 \mu \mathrm{L}, 2.75 \mathrm{mmol}), 352 \mathrm{mg}$ (65\%) of compound 1a were obtained after column chromatography. Pale yellow solid; ${ }^{1} \mathbf{H}$ NMR $\left(300 \mathrm{MHz}, \mathrm{CDCl}_{3}\right) \delta$ 8.42-8.38 (2H, m, Ar), 7.93 (1H, s, CH), 7.80-7.77 (2H, m, Ar), 7.51-7.46 (6H, m, Ar); ${ }^{13} \mathrm{C}$ NMR (75 MHz, $\left.\mathrm{CDCl}_{3}\right) \delta 149.1(\mathrm{C}), 134.7(\mathrm{CH}), 131.0(\mathrm{CH}), 130.6(\mathrm{C}), 129.9(\mathrm{CH})$, $129.2(\mathrm{CH}), 129.1(\mathrm{CH}), 128.6(\mathrm{CH}), 121.8(\mathrm{CH})$. 


\section{1-(4-Methoxyphenyl)- $N$-phenylmethanimine oxide (1b) ${ }^{4}$}

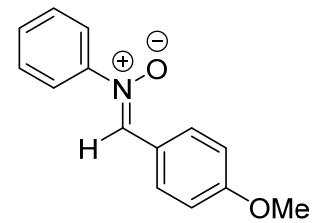

Procedure A. From N-phenylhydroxylamine (500 mg, $4.58 \mathrm{mmol})$ and 4methoxybenzaldehyde (557 $\mu \mathrm{L}, 4.58 \mathrm{mmol}), 414 \mathrm{mg}$ (40\%= of compound $\mathbf{1 b}$ were obtained. Yellow solid; ${ }^{1} \mathbf{H}$ NMR $\left(300 \mathrm{MHz}^{\mathrm{CDCl}} 3\right) \delta 8.40(2 \mathrm{H}, \mathrm{d}, J=$ $8.8 \mathrm{~Hz}, \mathrm{Ar}), 7.85$ (1H, s, CH), $7.76(2 \mathrm{H}, \mathrm{dd}, J=8.0,1.8 \mathrm{~Hz}, \mathrm{Ar}), 7.52-7.41$ $(3 \mathrm{H}, \mathrm{m}, \mathrm{Ar}), 6.98(2 \mathrm{H}, \mathrm{d}, J=8.8 \mathrm{~Hz}, \mathrm{Ar}), 3.87\left(3 \mathrm{H}, \mathrm{s}, \mathrm{CH}_{3}\right) ;{ }^{13} \mathrm{C}$ NMR $\left(75 \mathrm{MHz}, \mathrm{CDCl}_{3}\right) \delta 161.5$ (C), $148.9(\mathrm{C}), 134.1(\mathrm{CH}), 131.1(\mathrm{CH}), 129.6(\mathrm{CH}), 129.1(\mathrm{CH}), 123.7(\mathrm{C}), 121.6(\mathrm{CH}), 114.0$ $(\mathrm{CH}), 55.4(\mathrm{CH})$.

\section{1-(4-bromophenyl)- $N$-phenylmethanimine oxide (1c) ${ }^{4}$}

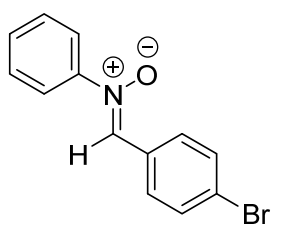

Procedure A. From $\mathrm{N}$-phenylhydroxylamine $(500 \mathrm{mg}, 4.58 \mathrm{mmol})$ and 4bromobenzaldehyde ( $847 \mathrm{mg}, 4.58 \mathrm{mmol}), 873 \mathrm{mg}$ (69\%) of compound 1c were obtained after filtration. White solid; ${ }^{1} \mathbf{H}$ NMR $\left(300 \mathrm{MHz}, \mathrm{CDCl}_{3}\right) \delta 8.28(2 \mathrm{H}, \mathrm{d}$, $J=8.6 \mathrm{~Hz}, \mathrm{Ar}), 7.89(1 \mathrm{H}, \mathrm{s}, \mathrm{CH}), 7.80-7.72(2 \mathrm{H}, \mathrm{m}, \mathrm{Ar}), 7.60(2 \mathrm{H}, \mathrm{d}, J=8.6 \mathrm{~Hz}$, Ar), 7.52-7.46 (3H, m, Ar); ${ }^{13} \mathrm{C}$ NMR (75 MHz, CDCl 3$) \delta 148.9(\mathrm{C}), 133.5(\mathrm{CH}), 131.9(\mathrm{CH})$, $130.3(\mathrm{CH}), 130.1(\mathrm{CH}), 129.5(\mathrm{C}), 129.2(\mathrm{CH}), 124.8(\mathrm{C}), 121.7(\mathrm{CH})$.

\section{1-(4-Nitrophenyl)- $N$-phenylmethanimine oxide (1d) ${ }^{4}$}

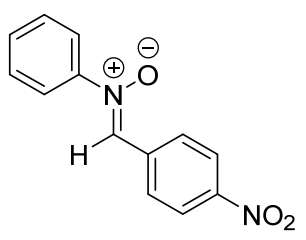

Procedure A. From $\mathrm{N}$-phenylhydroxylamine $(500 \mathrm{mg}, 4.58 \mathrm{mmol})$ and 4nitrobenzaldehyde (692 mg, $4.58 \mathrm{mmol}$ ), $1.101 \mathrm{~g} \mathrm{(98 \% )} \mathrm{of} \mathrm{compound} \mathrm{1d} \mathrm{were}$ obtained after filtration. Yellow solid; ${ }^{1} \mathbf{H}$ NMR $\left(300 \mathrm{MHz}, \mathrm{CDCl}_{3}\right) \delta 8.55(2 \mathrm{H}$, d, $J=9 \mathrm{~Hz}, \mathrm{Ar}), 8.32(2 \mathrm{H}, \mathrm{d}, J=9.0 \mathrm{~Hz}, \mathrm{Ar}), 8.07(1 \mathrm{H}, \mathrm{s}, \mathrm{CH}), 7.80-7.77(2 \mathrm{H}$, m, Ar), 7.54-7.51 (3H, m, Ar); ${ }^{13} \mathrm{C}$ NMR (75 MHz, $\left.\mathrm{CDCl}_{3}\right) \delta 148.9$ (C), $148.0(\mathrm{C}), 136.2(\mathrm{CH})$, $132.3(\mathrm{C}), 130.7(\mathrm{CH}), 129.4(\mathrm{CH}), 129.2(\mathrm{CH}), 123.9(\mathrm{CH}), 121.7(\mathrm{CH})$.

\section{$N$-Phenyl-1-(p-tolyl)methanimine oxide (1e) ${ }^{4}$}

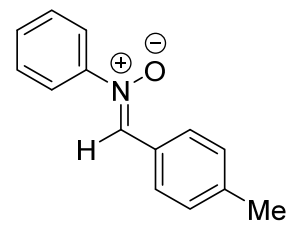

Procedure A. From $\mathrm{N}$-phenylhydroxylamine $(700 \mathrm{mg}, 6.41 \mathrm{mmol})$ and 4methylbenzaldehyde (756 $\mu \mathrm{L}, 6.41 \mathrm{mmol}), 908 \mathrm{mg}$ (67\%) of compound 1e were obtained after column chromatography. Pale yellow solid; ${ }^{1} \mathbf{H}$ NMR (300 MHz, $\left.\mathrm{CDCl}_{3}\right) \delta 8.30(2 \mathrm{H}, \mathrm{d}, J=8.4 \mathrm{~Hz}, \mathrm{Ar}), 7.89(1 \mathrm{H}, \mathrm{s}, \mathrm{CH}), 7.81-7.72(2 \mathrm{H}, \mathrm{m}, \mathrm{Ar})$, 7.52-7.41 (3H, m, Ar), $7.29(2 \mathrm{H}, J=8.4 \mathrm{~Hz}, \mathrm{Ar}), 2.41\left(3 \mathrm{H}, \mathrm{s}, \mathrm{CH}_{3}\right) ;{ }^{13} \mathrm{C}$ NMR $\left(75 \mathrm{MHz}, \mathrm{CDCl}_{3}\right) \delta$ $149.0(\mathrm{C}), 141.6(\mathrm{C}), 134.8(\mathrm{CH}), 129.8(\mathrm{CH}), 129.4(\mathrm{CH}), 129.13(\mathrm{CH}), 129.09(\mathrm{CH}), 128.0(\mathrm{C})$, $121.7(\mathrm{CH}), 21.8\left(\mathrm{CH}_{3}\right)$. 


\section{1-(3-Methoxyphenyl)- $N$-phenylmethanimine oxide (1f) ${ }^{4}$}

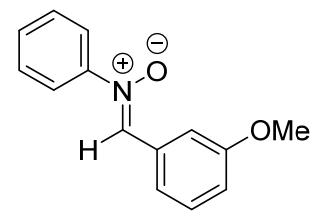

Procedure A. From N-phenylhydroxylamine (500 mg, $4.58 \mathrm{mmol})$ and 3methoxybenzaldehyde (558 $\mu \mathrm{L}, 4.58 \mathrm{mmol}), 934 \mathrm{mg}$ (90\%) of compound 1f were obtained after column chromatography. Orange oil; ${ }^{1} \mathbf{H}$ NMR $(300 \mathrm{MHz}$, $\left.\mathrm{CDCl}_{3}\right) \delta 8.37(1 \mathrm{H}, \mathrm{dd}, J=2.5,1.5 \mathrm{~Hz}, \mathrm{Ar}), 7.91(1 \mathrm{H}, \mathrm{s}, \mathrm{CH}), 7.80-7.73(2 \mathrm{H}$, m, Ar), $7.65(1 \mathrm{H}, \mathrm{d}, J=8.0 \mathrm{~Hz}, \mathrm{Ar}), 7.51-7.43(3 \mathrm{H}, \mathrm{m}, \mathrm{Ar}), 7.36(1 \mathrm{H}, \mathrm{t}, J=8.0 \mathrm{~Hz}, \mathrm{Ar}), 7.03(1 \mathrm{H}$, ddd, $J=8.0,2.5,1.5 \mathrm{~Hz}, \mathrm{Ar}), 3.87\left(3 \mathrm{H}, \mathrm{s}, \mathrm{CH}_{3}\right) ;{ }^{13} \mathbf{C} \mathbf{N M R}\left(75 \mathrm{MHz}, \mathrm{CDCl}_{3}\right) \delta 159.5(\mathrm{C}), 149.0$ (C), $134.6(\mathrm{CH}), 131.8(\mathrm{C}), 129.9(\mathrm{CH}), 129.4(\mathrm{CH}), 129.1(\mathrm{CH}), 122.2(\mathrm{CH}), 121.7(\mathrm{CH}), 117.9$ $(\mathrm{CH}), 112.5(\mathrm{CH}), 55.3\left(\mathrm{CH}_{3}\right)$.

\section{1-(3-Bromophenyl)- $N$-phenylmethanimine oxide (1g) ${ }^{4}$}<smiles>O=[N+](/C=C/c1cccc(Br)c1)c1ccccc1</smiles>

Procedure A. From N-phenylhydroxylamine (500 mg, $4.58 \mathrm{mmol})$ and 3bromobenzaldehyde (534 $\mu \mathrm{L}, 4.58 \mathrm{mmol}), 984 \mathrm{mg}$ (79\%) of compound $\mathbf{1 g}$ were obtained after filtration. Yellow solid; ${ }^{1} \mathbf{H}$ NMR $\left(300 \mathrm{MHz}, \mathrm{CDCl}_{3}\right) \delta 8.68(1 \mathrm{H}, \mathrm{t}$, $J=1.7 \mathrm{~Hz}, \mathrm{Ar}), 8.23(1 \mathrm{H}, \mathrm{d}, J=7.9 \mathrm{~Hz}, \mathrm{Ar}), 7.89(1 \mathrm{H}, \mathrm{s}, \mathrm{CH}), 7.77-7.73(2 \mathrm{H}, \mathrm{m}$, Ar), $7.58(1 \mathrm{H}, \mathrm{ddd}, J=8.0,2.0,1.0 \mathrm{~Hz}, \mathrm{Ar}), 7.52-7.45(3 \mathrm{H}, \mathrm{m}, \mathrm{Ar}), 7.34(1 \mathrm{H}, \mathrm{t}, J=8.0 \mathrm{~Hz}, \mathrm{Ar})$; ${ }^{13} \mathrm{C}$ NMR (75 MHz, $\left.\mathrm{CDCl}_{3}\right) \delta 148.9(\mathrm{C}), 133.7(\mathrm{CH}), 133.1(\mathrm{CH}), 132.4(\mathrm{C}), 131.3(\mathrm{CH}), 130.2$ $(\mathrm{CH}), 130.1(\mathrm{CH}), 129.2(\mathrm{CH}), 127.4(\mathrm{CH}), 122.8(\mathrm{C}), 121.7(\mathrm{CH})$.

\section{1-(3-Nitrophenyl)- $N$-phenylmethanimine oxide (1h) ${ }^{4}$}

Procedure A. From $N$-phenylhydroxylamine $(500 \mathrm{mg}, 4.58 \mathrm{mmol})$ and 3-<smiles>O=[N+]([O-])c1cccc(/C=[N+](\[O-])c2ccccc2)c1</smilesnitrobenzaldehyde (692 mg, $4.58 \mathrm{mmol}), 916$ (83\%) of compound $1 \mathrm{~h}$ were obtained after filtration. Yellow solid; ${ }^{1} \mathbf{H}$ NMR $\left(300 \mathrm{MHz}, \mathrm{CDCl}_{3}\right) \delta$ 9.21$9.17(1 \mathrm{H}, \mathrm{t}, J=1.8 \mathrm{~Hz}, \mathrm{Ar}), 8.82(1 \mathrm{H}, \mathrm{d}, J=8.0 \mathrm{~Hz}, \mathrm{Ar}), 8.29$ (1H, ddd, $J=$ 8.0, 1.8, 1.0 Hz, Ar), 8.07 (1H, s, CH), 7.83-7.75 (2H, m, Ar), 7.67 (1H, t, J = 8.0, Ar), 7.56-7.50 (3H, m, Ar); ${ }^{13} \mathrm{C}$ NMR (75 MHz, $\left.\mathrm{CDCl}_{3}\right) \delta 148.7$ (C), 148.3 (C), 133.8 (CH), 132.1 (C), 132.1 $(\mathrm{CH}), 130.6(\mathrm{CH}), 129.7(\mathrm{CH}), 129.4(\mathrm{CH}), 124.9(\mathrm{CH}), 123.4(\mathrm{CH}), 121.7(\mathrm{CH})$.

\section{1-(2-Methoxyphenyl)- $N$-phenylmethanimine oxide (1i) ${ }^{4}$}

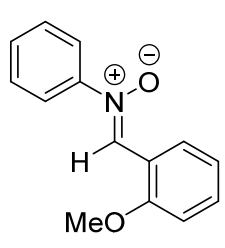

Procedure A. From $\mathrm{N}$-phenylhydroxylamine (124 mg, $1.14 \mathrm{mmol})$ and 2methoxybenzaldehyde (155.2 mg, $1.14 \mathrm{mmol}), 243 \mathrm{mg}$ (94\%) of compound 1i were obtained after column chromatography. Yellow pale solid; ${ }^{1} \mathbf{H}$ NMR (300 $\left.\mathrm{MHz}, \mathrm{CDCl}_{3}\right) \delta 9.48(1 \mathrm{H}, \mathrm{dd}, J=8.1,1.8 \mathrm{~Hz}, \mathrm{Ar}), 8.40(1 \mathrm{H}, \mathrm{s}, \mathrm{CH}), 7.80-7.77$ 
(2H, m, Ar), 7.50-7.39 (4H, m, Ar), 7.08 (1H, t, $J=7.8 \mathrm{~Hz}, \mathrm{Ar}), 6.92(1 \mathrm{H}, \mathrm{dd}, J=8.4,1.2 \mathrm{~Hz}, \mathrm{Ar})$; ${ }^{13}$ C NMR (75 MHz, $\left.\mathrm{CDCl}_{3}\right) \delta 157.4(\mathrm{C}), 149.5(\mathrm{C}), 132.1(\mathrm{CH}), 129.6(\mathrm{CH}), 129.3(\mathrm{C}), 129.0$ $(\mathrm{CH}), 128.7(\mathrm{CH}), 121.8(\mathrm{CH}), 120.8(\mathrm{CH}), 119.8(\mathrm{CH}), 109.8(\mathrm{CH}), 55.5\left(\mathrm{CH}_{3}\right)$.

\section{1-(2-Bromophenyl)- $N$-phenylmethanimine oxide $(1 \mathrm{j})^{4}$}

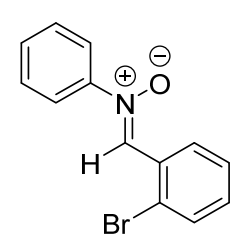

Procedure A. From N-phenylhydroxylamine (500 mg, $4.58 \mathrm{mmol}$ ) and 2methoxybenzaldehyde (535 $\mu \mathrm{L}, 4.58 \mathrm{mmol}), 1.15 \mathrm{~g}$ (91\%) of compound $\mathbf{1 j}$ were obtained. Yellow pale solid; ${ }^{1} \mathbf{H}$ NMR $\left(300 \mathrm{MHz}, \mathrm{CDCl}_{3}\right) \delta 9.51(1 \mathrm{H}, \mathrm{dd}, J=8.1$, $1.8 \mathrm{~Hz}, \mathrm{Ar}), 8.42(1 \mathrm{H}, \mathrm{s}, \mathrm{CH}), 7.81-7.77(2 \mathrm{H}, \mathrm{m}, \mathrm{Ar}), 7.66(1 \mathrm{H}, \mathrm{dd}, J=8.1,1.2 \mathrm{~Hz}$, Ar), 7.53-7.43 (4H, m, Ar), 7.29 (1H, td, $J=7.5,1.8 \mathrm{~Hz}, \mathrm{Ar}) ;{ }^{13} \mathbf{C}$ NMR $\left(75 \mathrm{MHz}, \mathrm{CDCl}_{3}\right) \delta 149.4$ (C), $133.1(\mathrm{CH}), 132.9(\mathrm{CH}), 131.8(\mathrm{CH}), 130.2(\mathrm{CH}), 129.7(\mathrm{C}), 129.5(\mathrm{CH}), 129.2(\mathrm{CH}), 127.8$ $(\mathrm{CH}), 124.1(\mathrm{C}), 121.8(\mathrm{CH})$.

\section{1-(2-nitrophenyl)- $N$-phenylmethanimine oxide $(1 \mathbf{k})^{4}$}

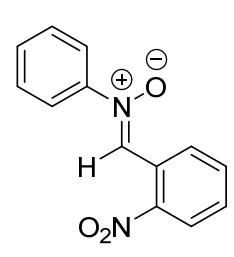

Procedure A. From $\mathrm{N}$-phenylhydroxylamine (500 $\mathrm{mg}, 4.58 \mathrm{mmol}$ ) and 3nitrobenzaldehyde (692 mg, $4.58 \mathrm{mmol}), 873 \mathrm{mg}$ (79\%) of compound 1k were obtained after filtration. Yellow solid; ${ }^{1} \mathbf{H}$ NMR $\left(300 \mathrm{MHz}, \mathrm{CDCl}_{3}\right) \delta 9.38(1 \mathrm{H}, \mathrm{dd}$, $J=8.1,1.5 \mathrm{~Hz}, \mathrm{Ar}), 8.59(1 \mathrm{H}, \mathrm{s}, \mathrm{CH}), 8.09$ (1H, dd, $J=8.1,1.2 \mathrm{~Hz}, \mathrm{Ar}), 7.81-7.74$ $(3 \mathrm{H}, \mathrm{m}, \mathrm{Ar}), 7.57(1 \mathrm{H}, \mathrm{dt}, J=8.4,1.5 \mathrm{~Hz}, \mathrm{Ar}), 7.53-7.49$ (3H, m, Ar); ${ }^{13} \mathrm{C}$ NMR $\left(75 \mathrm{MHz}, \mathrm{CDCl}_{3}\right)$ $\delta 149.2(\mathrm{C}), 147.5(\mathrm{C}), 133.6(\mathrm{CH}), 130.6(\mathrm{CH}), 130.5(\mathrm{CH}), 129.5(\mathrm{CH}), 129.3(\mathrm{CH}), 128.5(\mathrm{CH})$, $125.0(\mathrm{CH}), 124.5(\mathrm{C}), 121.8(\mathrm{CH})$.

\section{1-(Naphthalen-1-yl)- $N$-phenylmethanimine oxide (1l) ${ }^{4}$}

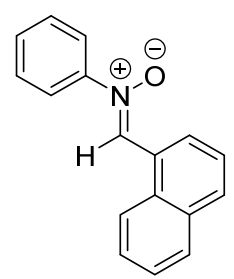

Procedure A. From $N$-phenylhydroxylamine $(360 \mathrm{mg}, 3.30 \mathrm{mmol})$ and 1naphthtaldehyde (450 $\mu \mathrm{L}, 3.30 \mathrm{mmol}, 334 \mathrm{mg}$ (41\%) of compound 11 were obtained after column chromatography. Yellow solid; ${ }^{1} \mathbf{H}$ NMR (300 MHz, $\left.\mathrm{CDCl}_{3}\right) \delta 9.78(1 \mathrm{H}, \mathrm{dd}, J=7.6,1.2 \mathrm{~Hz}, \mathrm{Ar}), 8.71(1 \mathrm{H}, \mathrm{s}, \mathrm{CH}), 8.09(1 \mathrm{H}, \mathrm{d}, J=7.4$ $\mathrm{Hz}, \mathrm{Ar}), 7.97(1 \mathrm{H}, \mathrm{d}, J=8.3 \mathrm{~Hz}, \mathrm{Ar}), 7.93(1 \mathrm{H}, \mathrm{dd}, J=7.8,1.7 \mathrm{~Hz}, \mathrm{Ar}), 7.88-7.82$ (2H, m, Ar), $7.64(1 \mathrm{H}, \mathrm{t}, J=7.8 \mathrm{~Hz}, \mathrm{Ar}), 7.60-7.48(5 \mathrm{H}, \mathrm{m}, \mathrm{Ar}) ;{ }^{13} \mathrm{C}$ NMR $\left(75 \mathrm{MHz}, \mathrm{CDCl}_{3}\right) \delta 149.9(\mathrm{C})$, $133.5(\mathrm{C}), 131.6(\mathrm{CH}), 130.9(\mathrm{C}), 130.5(\mathrm{CH}), 130.0(\mathrm{CH}), 129.4(\mathrm{CH}), 129.3(\mathrm{CH}), 127.03(\mathrm{CH})$, $127.02(\mathrm{CH}), 126.0(\mathrm{CH}), 125.8(\mathrm{CH}), 125.7(\mathrm{C}), 122.0(\mathrm{CH}), 121.7(\mathrm{CH})$. 


\section{$N$-Phenyl-1-(pyridin-2-yl)methanimine oxide (1m) ${ }^{4}$}

Procedure A. From $N$-phenylhydroxylamine $(500 \mathrm{mg}, 4.58 \mathrm{mmol})$ and picolinaldehyde (436 $\mu \mathrm{L}, 4.58 \mathrm{mmol}), 770 \mathrm{mg}(85 \%)$ of compound $\mathbf{1 m}$ were obtained after column chromatography. Yellow solid; ${ }^{1} \mathbf{H}$ NMR $\left(300 \mathrm{MHz}, \mathrm{CDCl}_{3}\right)$ $\delta 9.35(1 \mathrm{H}, \mathrm{d}, J=8.1 \mathrm{~Hz}, \mathrm{Ar}), 8.69$ (1H, ddd, $J=4.8,1.8,0.9 \mathrm{~Hz}, \mathrm{Ar}), 8.31(1 \mathrm{H}, \mathrm{s}$, $\mathrm{CH}), 7.92-7.79$ (3H, m, Ar), 7.54-7.46 (3H, m, Ar), $7.34(1 \mathrm{H}, \mathrm{ddd}, J=7.6,4.8,1.2 \mathrm{~Hz}, \mathrm{Ar}) ;{ }^{13} \mathrm{C}$ NMR $\left(75 \mathrm{MHz}, \mathrm{CDCl}_{3}\right) \delta 149.61(\mathrm{CH}), 149.60(\mathrm{C}), 148.7(\mathrm{C}), 137.1(\mathrm{CH}), 135.3(\mathrm{CH}), 130.4$ $(\mathrm{CH}), 129.2(\mathrm{CH}), 124.6(\mathrm{CH}), 124.0(\mathrm{CH}), 121.6(\mathrm{CH})$.

\section{1-Cyclohexyl- $N$-phenylmethanimine oxide (1n) ${ }^{4}$}

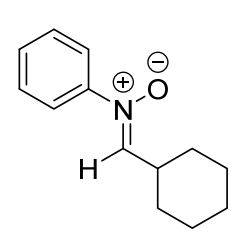

Procedure B. From $N$ - phenylhydroxylamine $(300 \mathrm{mg}, 2.74 \mathrm{mmol})$ and cyclohexanecarbaldehyde (330 $\mu \mathrm{L}, 2.74 \mathrm{mmol}), 192 \mathrm{mg}$ (35\%) of compound 1n were obtained after recrystallization from hexane:EtOAc. White solid; ${ }^{1} \mathbf{H}$ NMR $\left(300 \mathrm{MHz}, \mathrm{CDCl}_{3}\right) \delta$ 7.66-7.62 (2H, m, Ar), 7.43-7.39 (3H, m, Ar), $7.03(1 \mathrm{H}, \mathrm{d}, J=$ $7.5 \mathrm{~Hz}, \mathrm{CH}), 3.18(1 \mathrm{H}, \mathrm{m}, \mathrm{CH}), 2.03-1.98(2 \mathrm{H}, \mathrm{m}), 1.79-1.67(3 \mathrm{H}, \mathrm{m}), 1.52-1.34(2 \mathrm{H}, \mathrm{m}), 1.30-$ $7.21(3 \mathrm{H}, \mathrm{m}) ;{ }^{13} \mathrm{C}$ NMR (75 MHz, $\left.\mathrm{CDCl}_{3}\right) \delta 147.9(\mathrm{C}), 143.5(\mathrm{CH}), 129.7(\mathrm{CH}), 129.0(\mathrm{CH})$, $121.7(\mathrm{CH}), 35.6(\mathrm{CH}), 28.8\left(\mathrm{CH}_{2}\right), 25.9\left(\mathrm{CH}_{2}\right), 25.2\left(\mathrm{CH}_{2}\right)$.

\section{1-Cyclopropyl- $N$-phenylmethanimine oxide (10) ${ }^{4}$}

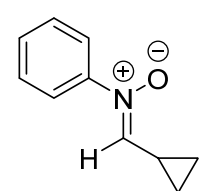

Procedure B. From $N$ - phenylhydroxylamine $(467 \mathrm{mg}, 4.28 \mathrm{mmol})$ and cyclohexanecarbaldehyde ( $320 \mu \mathrm{L}, 4.28 \mathrm{mmol}), 351 \mathrm{mg}(35 \%)$ of compound 10 were obtained after recrystallization from hexane:EtOAc. White solid; ${ }^{\mathbf{1}} \mathbf{H}$ NMR (300 $\left.\mathrm{MHz}, \mathrm{CDCl}_{3}\right) \delta$ 7.66-7.60 (2H, m, Ar), 7.44-7.36 (3H, m, Ar), $6.66(1 \mathrm{H}, \mathrm{d}, J=8.6 \mathrm{~Hz}, \mathrm{CH}), 2.62$ $(1 \mathrm{H}, \mathrm{qt}, J=8.5,4.9 \mathrm{~Hz}, \mathrm{CH}), 1.23-1.14(2 \mathrm{H}, \mathrm{m}), 0.86-0.78(2 \mathrm{H}, \mathrm{m}) ;{ }^{13} \mathrm{C}$ NMR $\left(75 \mathrm{MHz}, \mathrm{CDCl}_{3}\right) \delta$ $147.4(\mathrm{C}), 142.9(\mathrm{CH}), 129.6(\mathrm{CH}), 129.0(\mathrm{CH}), 121.4(\mathrm{CH}), 10.5(\mathrm{CH}), 7.75\left(\mathrm{CH}_{2}\right)$.

\section{$N$-(4-Chlorophenyl)-1-phenylmethanimine oxide (1p) ${ }^{4}$}

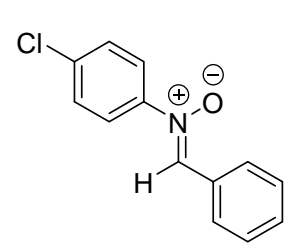

Procedure A. From N-(4-chlorophenyl)hydroxylamine (300 mg, $2.09 \mathrm{mmol})$ and benzaldehyde $(211 \mu \mathrm{L}, 2.09 \mathrm{mmol}), 386 \mathrm{mg}$ (79\%) of compound 1p were obtained after filtration. White solid; ${ }^{1} \mathbf{H}$ NMR $\left(300 \mathrm{MHz}, \mathrm{CDCl}_{3}\right) \delta 8.40-8.36$ $(2 \mathrm{H}, \mathrm{m}, \mathrm{Ar}), 7.91(1 \mathrm{H}, \mathrm{s}, \mathrm{CH}), 7.75(2 \mathrm{H}, \mathrm{d}, J=8.9 \mathrm{~Hz}, \mathrm{Ar}), 7.52-7.42$ (4H, m, Ar); ${ }^{13} \mathrm{C}$ NMR (75 MHz, $\left.\mathrm{CDCl}_{3}\right) \delta 147.4(\mathrm{C}), 135.8(\mathrm{C}), 134.8(\mathrm{CH}), 131.3(\mathrm{CH}), 130.4(\mathrm{C}), 129.3$ $(\mathrm{CH}), 129.2(\mathrm{CH}), 128.7(\mathrm{CH}), 123.0(\mathrm{CH})$. 


\section{$N$-(4-Methoxyphenyl)-1-phenylmethanimine oxide (1q) ${ }^{4}$}

Procedure A. From $N$-(4-methoxyphenyl)hydroxylamine (1.0 g, 7.2 mmol) and benzaldehyde (732 $\mu \mathrm{L}, 7.2 \mathrm{mmol}), 634 \mathrm{mg}$ (39\%) of compound 1q were obtained after column chromatography. Grey solid; ${ }^{1} \mathbf{H}$ NMR $(300 \mathrm{MHz}$, $\left.\mathrm{CDCl}_{3}\right) \delta$ 8.40-8.36 (2H, m, Ar), $7.87(1 \mathrm{H}, \mathrm{s}, \mathrm{CH}), 7.73(2 \mathrm{H}, \mathrm{d}, J=9.1 \mathrm{~Hz}, \mathrm{Ar})$, 7.50-7.44 (3H, m, Ar), $6.96(2 \mathrm{H}, \mathrm{d}, J=9.1 \mathrm{~Hz}, \mathrm{Ar}), 3.86\left(3 \mathrm{H}, \mathrm{s}, \mathrm{CH}_{3}\right) ;{ }^{13} \mathrm{C}$ NMR $(75 \mathrm{MHz}$, $\left.\mathrm{CDCl}_{3}\right) \delta 160.6(\mathrm{C}), 142.5(\mathrm{C}), 133.7(\mathrm{CH}), 130.8(\mathrm{C}), 130.7(\mathrm{CH}), 128.9(\mathrm{CH}), 128.6(\mathrm{CH}), 123.0$ $(\mathrm{CH}), 114.0(\mathrm{CH}), 55.6\left(\mathrm{CH}_{3}\right)$. 


\section{Synthesis and characterization data for compounds 3-8}

\section{General procedure for the asymmetric synthesis of 2-imidazolinones 3}

Squaramide SQ4 (14.6 mg, $0.025 \mathrm{mmol})$ and silver oxide $(3.0 \mathrm{mg}, 0.013 \mathrm{mmol})$ were introduced in a $25 \mathrm{~mL}$ round bottom flask followed by MTBE $(6 \mathrm{~mL})$ and nitrone $1(0.25 \mathrm{mmol})$. tert-Butyl isocyanoacetate $2 \mathbf{a}(48 \mu \mathrm{L}, 0.33 \mathrm{mmol})$ was added, the flask was closed with a stopper and the mixture was stirred at room temperature until consumption of the nitrone $\mathbf{1}$ (TLC). After this time, the reaction mixture was filtered through a short pad of silica gel and concentrated under reduced pressure. A small aliquot was analyzed by ${ }^{1} \mathrm{H}$ NMR to determinate the diastereomer ratio. The remaining crude was chromatographed on silica gel eluting with toluene:diethyl ether mixtures (9:1 to 5:5) to obtain the separated diastereomers trans-3 and cis-3.

Near racemic compounds were obtained by a similar procedure using an equimolar mixture of SQ1 and a similar squaramide derived from quinidine in place of SQ4. ${ }^{5}$

\section{tert-Butyl (4R,5S)-2-oxo-1,5-diphenylimidazolidine-4-carboxylate (3aa)}

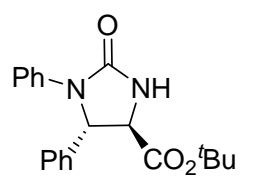

Obtained $66.2 \mathrm{mg}(78 \%)$ from $49.5 \mathrm{mg}(0.25 \mathrm{mmol})$ of 1a. The enantiomeric excess (minor isomer: 3\%, major isomer 90\%) was determined by HPLC (Amylose 1), hexane:iPrOH 80:20, $1 \mathrm{~mL} \cdot \mathrm{min}^{-1}$, cis-3aa (minor diastereomer): minor enantiomer, $\mathrm{t}_{\mathrm{r}}=9.0 \mathrm{~min}$, major enantiomer, $\mathrm{t}_{\mathrm{r}}=16.9 \mathrm{~min}$, trans-(4R,5S)-

3aa (major diastereomer): minor enantiomer $t_{r}=14.8 \mathrm{~min}$, major enantiomer, $t_{r}=23.2 \mathrm{~min}$.

trans-(4R,5S)-3aa (major diastereomer): white solid, m.p. $184-188{ }^{\circ} \mathrm{C}$; $[\alpha] \mathrm{D}^{25}-54.5($ c 0.95 , $\mathrm{CHCl}_{3}, 90 \%$ ee); ${ }^{1} \mathbf{H}$ NMR $\left(300 \mathrm{MHz}, \mathrm{CDCl}_{3}\right) \delta 7.44(2 \mathrm{H}, \mathrm{dd}, J=8.7,1.0 \mathrm{~Hz}, \mathrm{Ar}), 7.40-7.27(5 \mathrm{H}$, m, Ar), 7.25-7.18 (2H, m, Ar), 6.99 (1H, t, $J=7.4 \mathrm{~Hz}, \operatorname{Ar}), 5.85$ (1H, bs, NH), 5.43 (1H, d, $J=4.1$ $\mathrm{Hz}, \mathrm{CH}), 3.97(1 \mathrm{H}, \mathrm{d}, J=4.1 \mathrm{~Hz}, \mathrm{CH}), 1.53\left(9 \mathrm{H}, \mathrm{s}, \mathrm{CH}_{3}\right) ;{ }^{13} \mathrm{C} \mathbf{N M R}\left(75 \mathrm{MHz}, \mathrm{CDCl}_{3}\right) \delta 169.6(\mathrm{C})$, $158.4(\mathrm{C}), 139.7(\mathrm{C}), 138.3(\mathrm{C}), 129.1(\mathrm{CH}), 128.7(\mathrm{CH}), 128.3(\mathrm{CH}), 126.1(\mathrm{CH}), 123.4(\mathrm{CH})$, $120.1(\mathrm{CH}), 83.3(\mathrm{C}), 63.0(\mathrm{CH}), 60.3(\mathrm{CH}), 28.0\left(\mathrm{CH}_{3}\right)$; HRMS (ESI) m/z: $339.1703[\mathrm{M}+\mathrm{H}]^{+}$, $\mathrm{C}_{20} \mathrm{H}_{23} \mathrm{~N}_{2} \mathrm{O}_{3}{ }^{+}$requires 339.1703 .

cis-3aa (minor diastereomer): white solid, m.p. $198-203{ }^{\circ} \mathrm{C}$; $[\alpha]_{\mathrm{D}}{ }^{25}+3.8\left(\right.$ c $0.63, \mathrm{CHCl}_{3}, 3 \%$ ee); ${ }^{1} \mathbf{H}$ NMR $\left(300 \mathrm{MHz}, \mathrm{CDCl}_{3}\right) \delta 7.38(2 \mathrm{H}, \mathrm{dd}, J=8.8,1.1 \mathrm{~Hz}, \mathrm{Ar}), 7.35-7.23$ (5H, m, Ar), 7.22-7.15 $(2 \mathrm{H}, \mathrm{m}, \mathrm{Ar}) 6.96(1 \mathrm{H}, \mathrm{t}, J=7.4 \mathrm{~Hz}, \mathrm{Ar}), 5.50(1 \mathrm{H}, \mathrm{d}, J=9.6 \mathrm{~Hz}, \mathrm{CH}), 5.34(1 \mathrm{H}, \mathrm{bs}, \mathrm{NH}), 4.70(1 \mathrm{H}$, $\mathrm{dd}, J=9.6,0.9 \mathrm{~Hz}, \mathrm{CH}), 1.05\left(9 \mathrm{H}, \mathrm{s}, \mathrm{CH}_{3}\right) ;{ }^{13} \mathrm{C} \mathrm{NMR}\left(75 \mathrm{MHz}, \mathrm{CDCl}_{3}\right) \delta 167.5(\mathrm{C}), 158.6(\mathrm{C})$, $138.2(\mathrm{C}), 135.9(\mathrm{C}), 128.7(\mathrm{CH}), 128.61(\mathrm{CH}), 128.59(\mathrm{CH}), 128.0(\mathrm{CH}), 123.5(\mathrm{CH}), 120.5(\mathrm{CH})$, $82.6(\mathrm{C}), 62.6(\mathrm{CH}), 57.5(\mathrm{CH}), 27.3\left(\mathrm{CH}_{3}\right)$; HRMS (ESI) m/z: $339.1703[\mathrm{M}+\mathrm{H}]^{+}, \mathrm{C}_{20} \mathrm{H}_{23} \mathrm{~N}_{2} \mathrm{O}_{3}{ }^{+}$ requires 339.1703 . 


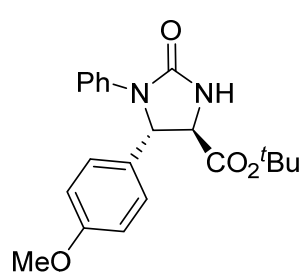

min, major enantiomer, $\mathrm{t}_{\mathrm{r}}=36.4 \mathrm{~min}$.

trans-(4R,5S)-3ba (major diastereomer): white solid, m.p. $173-178{ }^{\circ} \mathrm{C} ;[\alpha]_{\mathrm{D}}^{25}-40.6$ (c 0.97 , $\mathrm{CHCl}_{3}, 89 \%$ ee); ${ }^{1} \mathbf{H}$ NMR $\left(300 \mathrm{MHz}, \mathrm{CDCl}_{3}\right) \delta 7.42(2 \mathrm{H}, \mathrm{dd}, J=8.7,1.1 \mathrm{~Hz}, \mathrm{Ar}), 7.29(2 \mathrm{H}, \mathrm{d}, J$ $=8.7 \mathrm{~Hz}, \mathrm{Ar}), 7.25-7.18(2 \mathrm{H}, \mathrm{m}, \mathrm{Ar}), 6.99(1 \mathrm{H}, \mathrm{t}, J=7.4 \mathrm{~Hz}, \operatorname{Ar}), 6.86(2 \mathrm{H}, \mathrm{d}, J=8.8 \mathrm{~Hz}, \mathrm{Ar})$, $5.62(1 \mathrm{H}, \mathrm{bs}, \mathrm{NH}), 5.37(1 \mathrm{H}, \mathrm{d}, J=4.3 \mathrm{~Hz}, \mathrm{CH}), 3.95(1 \mathrm{H}, \mathrm{d}, J=4.3 \mathrm{~Hz}, \mathrm{CH}), 3.76\left(3 \mathrm{H}, \mathrm{s}, \mathrm{CH}_{3}\right)$, $1.51\left(9 \mathrm{H}, \mathrm{s}, \mathrm{CH}_{3}\right) ;{ }^{13} \mathrm{C}$ NMR (75 MHz, $\left.\mathrm{CDCl}_{3}\right) \delta 169.7$ (C), 159.5 (C), 158.3 (C), 138.3 (C), 131.6 (C), $128.6(\mathrm{CH}), 127.4(\mathrm{CH}), 123.5(\mathrm{CH}), 120.4(\mathrm{CH}), 114.4(\mathrm{CH}), 83.2(\mathrm{C}), 62.7(\mathrm{CH}), 60.5(\mathrm{CH})$, $55.2\left(\mathrm{CH}_{3}\right), 28.0\left(\mathrm{CH}_{3}\right)$; HRMS (ESI) m/z: $369.1808[\mathrm{M}+\mathrm{H}]^{+}, \mathrm{C}_{21} \mathrm{H}_{25} \mathrm{~N}_{2} \mathrm{O}_{4}{ }^{+}$requires 369.1809.

cis-3ba (minor diastereomer): white solid, m.p. $194-197^{\circ} \mathrm{C}$; $[\alpha]_{\mathrm{D}}{ }^{25}+5.8$ (c 0.44, $\mathrm{CHCl}_{3}, 16 \%$ ee); ${ }^{1} \mathbf{H}$ NMR $\left(300 \mathrm{MHz}, \mathrm{CDCl}_{3}\right) \delta 7.38(2 \mathrm{H}, \mathrm{dd}, J=8.7,1.1 \mathrm{~Hz}, \mathrm{Ar}), 7.24(2 \mathrm{H}, \mathrm{d}, J=9.4 \mathrm{~Hz}, \mathrm{Ar})$, 7.22-7.14 (2H, m, Ar), $6.97(1 \mathrm{H}, \mathrm{t}, J=7.4 \mathrm{~Hz}, \mathrm{Ar}), 6.80(2 \mathrm{H}, \mathrm{d}, J=8.8 \mathrm{~Hz}, \mathrm{Ar}), 5.45(1 \mathrm{H}, \mathrm{d}, J=$ $9.5 \mathrm{~Hz}, \mathrm{CH}), 5.19(1 \mathrm{H}, \mathrm{bs}, \mathrm{NH}), 4.67(1 \mathrm{H}, \mathrm{d}, J=9.5 \mathrm{~Hz}, \mathrm{CH}), 3.74\left(3 \mathrm{H}, \mathrm{s}, \mathrm{CH}_{3}\right), 1.10(9 \mathrm{H}, \mathrm{s}$, $\left.\mathrm{CH}_{3}\right) ;{ }^{13} \mathrm{C}$ NMR $\left(75 \mathrm{MHz}, \mathrm{CDCl}_{3}\right) \delta 167.6(\mathrm{C}), 159.8(\mathrm{C}), 158.5(\mathrm{C}), 138.2(\mathrm{C}), 129.2(\mathrm{CH}), 128.6$ $(\mathrm{CH}), 127.9(\mathrm{C}), 123.5(\mathrm{CH}), 120.5(\mathrm{CH}), 114.0(\mathrm{CH}), 82.5(\mathrm{C}), 62.2(\mathrm{CH}), 57.7(\mathrm{CH}), 55.3\left(\mathrm{CH}_{3}\right)$, $27.5\left(\mathrm{CH}_{3}\right)$; HRMS (ESI) m/z: $369.1808[\mathrm{M}+\mathrm{H}]^{+}, \mathrm{C}_{21} \mathrm{H}_{25} \mathrm{~N}_{2} \mathrm{O}_{4}{ }^{+}$requires 369.1809 .

tert-Butyl (4R,5S)-5-(4-bromophenyl)-2-oxo-1-phenylimidazolidine-4-carboxylate (3ca)

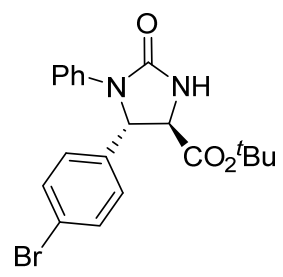

Obtained $68.5 \mathrm{mg}(65 \%)$ from $69.3 \mathrm{mg}(0.25 \mathrm{mmol})$ of 1c. The enantiomeric excess (minor isomer: 6\%, major isomer 86\%) was determined by HPLC (Amylose 1), hexane:iPrOH 80:20, $1 \mathrm{~mL} \cdot \mathrm{min}^{-1}$, cis-3ca (minor diastereomer): minor enantiomer, $\mathrm{t}_{\mathrm{r}}=7.4 \mathrm{~min}$, major enantiomer, $\mathrm{t}_{\mathrm{r}}=9.6 \mathrm{~min}$, trans-(4R,5S)-3ca (major diastereomer): minor enantiomer, $\mathrm{t}_{\mathrm{r}}=13.7 \mathrm{~min}$, major enantiomer, $\mathrm{t}_{\mathrm{r}}=19.3 \mathrm{~min}$.

trans-(4R,5S)-3ca(major diastereomer): white solid, m.p. $163-164{ }^{\circ} \mathrm{C}$; $[\alpha]_{\mathrm{D}}^{25}-37.4$ (c 0.86 , $\mathrm{CHCl}_{3}, 86 \%$ ee); ${ }^{1} \mathbf{H}$ NMR $\left(300 \mathrm{MHz}, \mathrm{CDCl}_{3}\right) \delta 7.47(2 \mathrm{H}, \mathrm{d}, J=8.5 \mathrm{~Hz}, \mathrm{Ar}), 7.39(2 \mathrm{H}, \mathrm{dd}, J=8.8$, 1.1 Hz, Ar), 7.29-7.15 (4H, m, Ar), $7.01(1 \mathrm{H}, \mathrm{t}, J=7.4 \mathrm{~Hz}, \mathrm{Ar}), 5.97(1 \mathrm{H}, \mathrm{bs}, \mathrm{NH}), 5.39$ (1H, d, $J$ $=4.3 \mathrm{~Hz}, \mathrm{CH}), 3.93(1 \mathrm{H}, \mathrm{d}, J=4.3 \mathrm{~Hz}, \mathrm{CH}), 1.52\left(9 \mathrm{H}, \mathrm{s}, \mathrm{CH}_{3}\right) ;{ }^{13} \mathbf{C} \mathbf{N M R}\left(75 \mathrm{MHz}, \mathrm{CDCl}_{3}\right) \delta$ 
$169.3(\mathrm{C}), 158.3$ (C), 138.7 (C), $138.0(\mathrm{C}), 132.3(\mathrm{CH}), 128.8(\mathrm{CH}), 127.9(\mathrm{CH}), 123.7(\mathrm{CH})$, $122.3(\mathrm{C}), 120.2(\mathrm{CH}), 83.5(\mathrm{C}), 62.4(\mathrm{CH}), 60.1(\mathrm{CH}), 28.0\left(\mathrm{CH}_{3}\right)$; HRMS (ESI) m/z: 417.0801 $[\mathrm{M}+\mathrm{H}]^{+}, \mathrm{C}_{20} \mathrm{H}_{21} \mathrm{BrN}_{2} \mathrm{O}_{3}{ }^{+}$requires 417.0808 .

cis-3ca (minor diastereomer): white solid, m.p. $199-202{ }^{\circ} \mathrm{C}$; $[\alpha]_{\mathrm{D}}{ }^{25}+5.5\left(\right.$ c $0.63, \mathrm{CHCl}_{3}, 6 \%$ ee); ${ }^{1} \mathbf{H}$ NMR $\left(300 \mathrm{MHz}, \mathrm{CDCl}_{3}\right) \delta 7.42(2 \mathrm{H}, \mathrm{d}, J=8.5 \mathrm{~Hz}, \mathrm{Ar}), 7.35(2 \mathrm{H}, \mathrm{dd}, J=8.6,1.0 \mathrm{~Hz}, \mathrm{Ar})$, 7.23-7.18 (4H, m, Ar), $6.99(1 \mathrm{H}, \mathrm{t}, J=7.3 \mathrm{~Hz}, \mathrm{Ar}), 5.47(1 \mathrm{H}, \mathrm{d}, J=9.6 \mathrm{~Hz}, \mathrm{CH}), 5.32(1 \mathrm{H}, \mathrm{bs}$, $\mathrm{NH}), 4.69(1 \mathrm{H}, \mathrm{d}, J=9.6 \mathrm{~Hz}, \mathrm{CH}), 1.10\left(9 \mathrm{H}, \mathrm{s}, \mathrm{CH}_{3}\right) ;{ }^{13} \mathrm{C} \mathrm{NMR}\left(75 \mathrm{MHz}, \mathrm{CDCl}_{3}\right) \delta 167.4(\mathrm{C})$, $158.4(\mathrm{C}), 137.9(\mathrm{C}), 134.9(\mathrm{C}), 131.8(\mathrm{CH}), 129.7(\mathrm{CH}), 128.8(\mathrm{CH}), 123.9(\mathrm{CH}), 122.7(\mathrm{C})$, $120.6(\mathrm{CH}), 82.9(\mathrm{C}), 62.0(\mathrm{CH}), 57.3(\mathrm{CH}), 27.4\left(\mathrm{CH}_{3}\right)$; ); HRMS (ESI) m/z: $417.0801[\mathrm{M}+\mathrm{H}]^{+}$, $\mathrm{C}_{20} \mathrm{H}_{21} \mathrm{BrN}_{2} \mathrm{O}_{3}{ }^{+}$requires 417.0808 .

\section{tert-Butyl (4R,5S)-5-(4-nitrophenyl)-2-oxo-1-phenylimidazolidine-4-carboxylate (3da)}

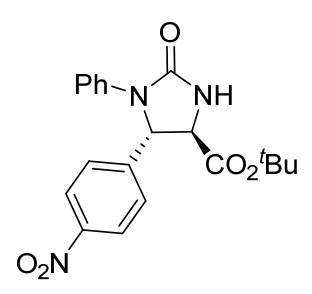

Obtained $58.2 \mathrm{mg}(60 \%)$ from $60.8 \mathrm{mg}(0.25 \mathrm{mmol})$ of $\mathbf{1 d}$. The enantiomeric excess (minor isomer: 10\%, major isomer 87\%) was determined by HPLC (lux Cellulose 3), hexane:iPrOH 95:5, $1.5 \mathrm{~mL} \cdot \mathrm{min}^{-1}$, cis-3da (minor diastereomer): minor enantiomer, $\mathrm{t}_{\mathrm{r}}=20.5 \mathrm{~min}$, major enantiomer, $\mathrm{t}_{\mathrm{r}}=15.4$ min, trans-(4R,5S)-3da (major diastereomer): minor enantiomer, $\mathrm{tr}_{\mathrm{r}}=26.8$ $\min$, major enantiomer, $\mathrm{t}_{\mathrm{r}}=28.5 \mathrm{~min}$.

trans-(4R,5S)-3da (major diastereomer): brown oil; $[\alpha]_{\mathrm{D}}{ }^{25}-26.6$ (c $0.81, \mathrm{CHCl}_{3}, \%$ ee); ${ }^{1} \mathbf{H}$ NMR $\left(300 \mathrm{MHz}, \mathrm{CDCl}_{3}\right) \delta 8.21(2 \mathrm{H}, \mathrm{d}, J=8.8 \mathrm{~Hz}, \mathrm{Ar}), 7.57(2 \mathrm{H}, \mathrm{d}, J=8.7 \mathrm{~Hz}, \mathrm{Ar}), 7.38(2 \mathrm{H}, \mathrm{dd}$, $J=8.7,1.1 \mathrm{~Hz}, \mathrm{Ar}), 7.24-7.12(2 \mathrm{H}, \mathrm{m}, \mathrm{Ar}), 7.02(1 \mathrm{H}, \mathrm{t}, J=7.4 \mathrm{~Hz}, \mathrm{Ar}), 5.96(1 \mathrm{H}, \mathrm{bs}, \mathrm{NH}), 5.57$ $(1 \mathrm{H}, \mathrm{d}, J=4.4 \mathrm{~Hz}, \mathrm{CH}), 3.95(1 \mathrm{H}, \mathrm{d}, J=4.4 \mathrm{~Hz}, \mathrm{CH}), 1.54\left(9 \mathrm{H}, \mathrm{s}, \mathrm{CH}_{3}\right) ;{ }^{13} \mathrm{C} \mathbf{N M R}(75 \mathrm{MHz}$, $\left.\mathrm{CDCl}_{3}\right) \delta 168.9(\mathrm{C}), 158.0(\mathrm{C}), 147.9(\mathrm{C}), 146.7(\mathrm{C}), 137.6(\mathrm{C}), 129.0(\mathrm{CH}), 127.3(\mathrm{CH}), 124.4$ $(\mathrm{CH}), 124.1(\mathrm{CH}), 120.2(\mathrm{CH}), 84.0(\mathrm{C}), 62.2(\mathrm{CH}), 59.7(\mathrm{CH}), 28.0\left(\mathrm{CH}_{3}\right)$; HRMS (ESI) m/z: $384.1549[\mathrm{M}+\mathrm{H}]^{+}, \mathrm{C}_{20} \mathrm{H}_{22} \mathrm{~N}_{3} \mathrm{O}_{5}{ }^{+}$requires 384.1554 .

cis-3da (minor diastereomer): white solid, m.p. $199-207^{\circ} \mathrm{C}$; $[\alpha]_{\mathrm{D}}{ }^{25}+2.1\left(\right.$ c $0.54, \mathrm{CHCl}_{3}, 10 \%$ ee); ${ }^{1} \mathbf{H}$ NMR $\left(300 \mathrm{MHz}, \mathrm{CDCl}_{3}\right) \delta 8.16(2 \mathrm{H}, \mathrm{d}, J=8.8 \mathrm{~Hz}, \mathrm{Ar}), 7.54(2 \mathrm{H}, \mathrm{d}, J=8.8 \mathrm{~Hz}, \mathrm{Ar}), 7.33(2 \mathrm{H}$, $\mathrm{dd}, J=8.6,0.9 \mathrm{~Hz}, \mathrm{Ar}), 7.21(2 \mathrm{H}, \mathrm{t}, J=8.0 \mathrm{~Hz}, \mathrm{Ar}), 7.01(1 \mathrm{H}, \mathrm{t}, J=7.3 \mathrm{~Hz}, \mathrm{Ar}), 5.63(1 \mathrm{H}, \mathrm{d}, J$ $=9.6 \mathrm{~Hz}, \mathrm{CH}), 5.41(1 \mathrm{H}, \mathrm{bs}, \mathrm{NH}), 4.76(1 \mathrm{H}, \mathrm{d}, J=9.6 \mathrm{~Hz}, \mathrm{CH}), 1.08\left(9 \mathrm{H}, \mathrm{s}, \mathrm{CH}_{3}\right) ;{ }^{13} \mathrm{C}$ NMR $(75$ $\left.\mathrm{MHz}, \mathrm{CDCl}_{3}\right) \delta 167.0(\mathrm{C}), 158.2(\mathrm{C}), 148.1(\mathrm{C}), 143.2(\mathrm{C}), 137.5(\mathrm{C}), 129.2(\mathrm{CH}), 128.9(\mathrm{CH})$, $124.3(\mathrm{CH}), 123.8(\mathrm{CH}), 120.6(\mathrm{CH}), 83.3(\mathrm{C}), 61.8(\mathrm{CH}), 57.1(\mathrm{CH}), 27.5\left(\mathrm{CH}_{3}\right)$; HRMS (ESI) $\mathrm{m} / \mathrm{z}: 384.1549[\mathrm{M}+\mathrm{H}]^{+}, \mathrm{C}_{20} \mathrm{H}_{22} \mathrm{~N}_{3} \mathrm{O}_{5}{ }^{+}$requires 384.1554 . 


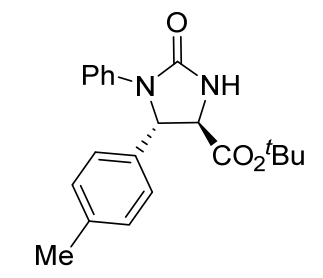

Obtained $73.7 \mathrm{mg}(83 \%)$ from $53.0 \mathrm{mg}(0.25 \mathrm{mmol})$ of 1e. The enantiomeric excess (minor isomer: 7\%, major isomer 90\%) was determined by HPLC (Amylose 1), hexane:iPrOH 80:20, $1 \mathrm{~mL} \cdot \min ^{-1}$, cis-3b (minor diastereomer): minor enantiomer, $\mathrm{t}_{\mathrm{r}}=6.9 \mathrm{~min}$, major enantiomer, $\mathrm{t}_{\mathrm{r}}=10.6$ min, trans-(4R,5S)-3b (major diastereomer): minor enantiomer $t_{r}=12.4$ min, major enantiomer, $\mathrm{t}_{\mathrm{r}}=21.9 \mathrm{~min}$.

trans-(4R,5S)-3ea (major diastereomer): white solid, m.p. $176-181{ }^{\circ} \mathrm{C} ;[\alpha]_{\mathrm{D}}{ }^{25}-46.2$ (c 0.71 , $\mathrm{CHCl}_{3}, 90 \%$ ee); ${ }^{1} \mathbf{H}$ NMR $\left(300 \mathrm{MHz}, \mathrm{CDCl}_{3}\right) \delta 7.43(2 \mathrm{H}, \mathrm{dd}, J=8.8,1.1 \mathrm{~Hz}, \mathrm{Ar}), 7.30-7.18(4 \mathrm{H}$, m, Ar), $7.14(2 \mathrm{H}, \mathrm{d}, J=7.9 \mathrm{~Hz}, \mathrm{Ar}), 6.99(1 \mathrm{H}, \mathrm{t}, J=7.4 \mathrm{~Hz}, \mathrm{Ar}), 5.39$ (1H, d, $J=4.1 \mathrm{~Hz}, \mathrm{CH})$, $3.94(1 \mathrm{H}, \mathrm{d}, J=4.1 \mathrm{~Hz}, \mathrm{CH}), 2.31\left(3 \mathrm{H}, \mathrm{s}, \mathrm{CH}_{3}\right), 1.52\left(9 \mathrm{H}, \mathrm{s}, \mathrm{CH}_{3}\right) ;{ }^{13} \mathbf{C}$ NMR $\left(75 \mathrm{MHz}, \mathrm{CDCl}_{3}\right) \delta$ $169.7(\mathrm{C}), 158.3(\mathrm{C}), 138.3(\mathrm{C}), 138.2(\mathrm{C}), 129.8(\mathrm{CH}), 128.7(\mathrm{CH}), 126.1(\mathrm{CH}), 123.5(\mathrm{CH})$, $120.2(\mathrm{CH}), 83.3(\mathrm{C}), 62.9(\mathrm{CH}), 60.4(\mathrm{CH}), 28.0\left(\mathrm{CH}_{3}\right), 21.1\left(\mathrm{CH}_{3}\right)$; HRMS (ESI) m/z: 353.1855, $[\mathrm{M}+\mathrm{H}]^{+}, \mathrm{C}_{21} \mathrm{H}_{25} \mathrm{~N}_{2} \mathrm{O}_{3}{ }^{+}$requires 353.1860 .

cis-3ea (minor diastereomer): white solid, m.p. $197-201{ }^{\circ} \mathrm{C}$; $[\alpha]_{\mathrm{D}}{ }^{25}+4.3\left(\right.$ c $0.74, \mathrm{CHCl}_{3}, 7 \%$ ee); ${ }^{1} \mathbf{H}$ NMR (300 MHz, $\left.\mathrm{CDCl}_{3}\right) \delta 7.39(2 \mathrm{H}, \mathrm{dd}, J=8.8,1.1 \mathrm{~Hz}, \mathrm{Ar}), 7.22-7.16(4 \mathrm{H}, \mathrm{m}, \mathrm{Ar}), 7.07$ (2H, $\mathrm{d}, J=7.9 \mathrm{~Hz}, \mathrm{Ar}), 6.96(1 \mathrm{H}, \mathrm{t}, J=7.4 \mathrm{~Hz}, \mathrm{Ar}), 5.46(1 \mathrm{H}, \mathrm{d}, J=9.6 \mathrm{~Hz}, \mathrm{CH}), 5.35(1 \mathrm{H}, \mathrm{bs}, \mathrm{NH})$, $4.68(1 \mathrm{H}, \mathrm{d}, J=9.6 \mathrm{~Hz}, \mathrm{CH}), 2.27\left(3 \mathrm{H}, \mathrm{s}, \mathrm{CH}_{3}\right), 1.06\left(9 \mathrm{H}, \mathrm{s}, \mathrm{CH}_{3}\right) ;{ }^{13} \mathrm{C} \mathbf{N M R}\left(75 \mathrm{MHz}, \mathrm{CDCl}_{3}\right) \delta$ $167.6(\mathrm{C}), 158.6(\mathrm{C}), 138.4(\mathrm{C}), 138.3(\mathrm{C}), 132.8(\mathrm{C}), 129.2(\mathrm{CH}), 128.6(\mathrm{CH}), 127.9(\mathrm{CH}), 123.4$ $(\mathrm{CH}), 120.5(\mathrm{CH}), 82.5(\mathrm{C}), 62.4(\mathrm{CH}), 57.6(\mathrm{CH}), 27.3\left(\mathrm{CH}_{3}\right), 21.05\left(\mathrm{CH}_{3}\right)$; HRMS (ESI) m/z: $353.1855[\mathrm{M}+\mathrm{H}]^{+}, \mathrm{C}_{21} \mathrm{H}_{25} \mathrm{~N}_{2} \mathrm{O}_{3}{ }^{+}$requires 353.1860 .

tert-Butyl (4R,5S)-5-(3-methoxyphenyl)-2-oxo-1-phenylimidazolidine-4-carboxylate (3fa)

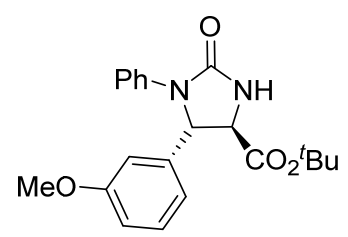

Obtained $58.6 \mathrm{mg}(63 \%)$ from $57.0 \mathrm{mg}(0.25 \mathrm{mmol})$ of 1f. The enantiomeric excess (minor isomer: 6\%, major isomer 89\%) was determined by HPLC (Chiracel OD-H), hexane:iPrOH 90:10, $1 \mathrm{~mL} \cdot \mathrm{min}^{-1}$, cis-3fa (minor diastereomer): minor enantiomer, $\mathrm{t}_{\mathrm{r}}=23.1 \mathrm{~min}$, major enantiomer, $\mathrm{t}_{\mathrm{r}}=27.2$ $\min$, trans-(4R,5S)-3fa (major diastereomer): minor enantiomer, $\mathrm{t}_{\mathrm{r}}=13.2 \mathrm{~min}$, major enantiomer, $\mathrm{t}_{\mathrm{r}}=14.9 \mathrm{~min}$.

trans-(4R,5S)-3fa (major diastereomer): white solid, m.p. 162-166 ${ }^{\circ} \mathrm{C} ;[\alpha]_{\mathrm{D}}{ }^{25}-45.7$ (c 0.95 , $\mathrm{CHCl}_{3}, 89 \%$ ee); ${ }^{1} \mathbf{H}$ NMR $\left(300 \mathrm{MHz}, \mathrm{CDCl}_{3}\right) \delta 7.44(2 \mathrm{H}, \mathrm{dd}, J=8.7,1.0 \mathrm{~Hz}, \mathrm{Ar}), 7.27-7.20(3 \mathrm{H}$, m, Ar), 7.01-6.95 (2H, m, Ar), $6.90(1 \mathrm{H}, \mathrm{t}, J=1.5 \mathrm{~Hz}, \mathrm{Ar}), 6.81$ (1H, ddd, $J=8.2,2.5,0.8 \mathrm{~Hz}, \mathrm{Ar})$, $5.69(1 \mathrm{H}, \mathrm{bs}, \mathrm{NH}), 5.39(1 \mathrm{H}, \mathrm{d}, J=4.1 \mathrm{~Hz}, \mathrm{CH}), 3.96(1 \mathrm{H}, \mathrm{d}, J=4.1 \mathrm{~Hz}, \mathrm{CH}), 3.76\left(3 \mathrm{H}, \mathrm{s}, \mathrm{CH}_{3}\right)$, 
$1.52\left(9 \mathrm{H}, \mathrm{s}, \mathrm{CH}_{3}\right) ;{ }^{13} \mathrm{C}$ NMR $\left(75 \mathrm{MHz}, \mathrm{CDCl}_{3}\right) \delta 169.6(\mathrm{C}), 160.2$ (C), 158.3 (C), 141.4 (C), 138.3 (C), $130.2(\mathrm{CH}), 128.7(\mathrm{CH}), 123.5(\mathrm{CH}), 120.1(\mathrm{CH}), 118.3(\mathrm{CH}), 113.7(\mathrm{CH}), 111.6(\mathrm{CH}), 83.3$ (C), $62.9(\mathrm{CH}), 60.2(\mathrm{CH}), 55.2\left(\mathrm{CH}_{3}\right), 28.0\left(\mathrm{CH}_{3}\right)$; HRMS (ESI) m/z: $369.1807[\mathrm{M}+\mathrm{H}]^{+}$, $\mathrm{C}_{21} \mathrm{H}_{25} \mathrm{~N}_{2} \mathrm{O}_{4}{ }^{+}$requires 369.1809 .

cis-3fa (minor diastereomer): white solid, m.p. $172-175^{\circ} \mathrm{C}$; $[\alpha]_{\mathrm{D}}{ }^{25}-1.1$ (c $0.61, \mathrm{CHCl}_{3}, 6 \%$ ee); ${ }^{1} \mathbf{H}$ NMR $\left(300 \mathrm{MHz}, \mathrm{CDCl}_{3}\right) \delta 7.38(2 \mathrm{H}, \mathrm{dd}, J=8.7,1.1 \mathrm{~Hz}, \mathrm{Ar}), 7.23-7.16(3 \mathrm{H}, \mathrm{m}, \mathrm{Ar}), 6.98(1 \mathrm{H}$, t, $J=7.4 \mathrm{~Hz}, \mathrm{Ar}), 6.92(1 \mathrm{H}, \mathrm{d}, J=7.7 \mathrm{~Hz}, \mathrm{Ar}), 6.85-6.84(1 \mathrm{H}, \mathrm{m}, \mathrm{Ar}), 6.78(1 \mathrm{H}, \mathrm{ddd}, J=8.2,2.5$, $0.8 \mathrm{~Hz}, \mathrm{Ar}), 5.47(1 \mathrm{H}, \mathrm{d}, J=9.7 \mathrm{~Hz}, \mathrm{CH}), 5.31(1 \mathrm{H}, \mathrm{bs}, \mathrm{NH}), 4.68(1 \mathrm{H}, \mathrm{d}, J=9.7 \mathrm{~Hz}, \mathrm{CH}), 3.73$ $\left(3 \mathrm{H}, \mathrm{s}, \mathrm{CH}_{3}\right), 1.08\left(9 \mathrm{H}, \mathrm{s}, \mathrm{CH}_{3}\right) ;{ }^{13} \mathrm{C}$ NMR $\left(75 \mathrm{MHz}, \mathrm{CDCl}_{3}\right) \delta 167.6(\mathrm{C}), 159.7$ (C), $158.6(\mathrm{C})$, $138.2(\mathrm{C}), 137.3(\mathrm{CH}), 129.6(\mathrm{CH}), 128.6(\mathrm{CH}), 123.6(\mathrm{CH}), 120.6(\mathrm{CH}), 120.4(\mathrm{C}), 114.3(\mathrm{CH})$, $113.5(\mathrm{CH}), 82.5(\mathrm{C}), 62.6(\mathrm{CH}), 57.5(\mathrm{CH}), 55.2\left(\mathrm{CH}_{3}\right), 27.4\left(\mathrm{CH}_{3}\right)$; HRMS (ESI) m/z:369.1807 $[\mathrm{M}+\mathrm{H}]^{+}, \mathrm{C}_{21} \mathrm{H}_{25} \mathrm{~N}_{2} \mathrm{O}_{4}{ }^{+}$requires 369.1809 .

tert-Butyl (4R,5S)-5-(3-bromophenyl)-2-oxo-1-phenylimidazolidine-4-carboxylate (3ga)

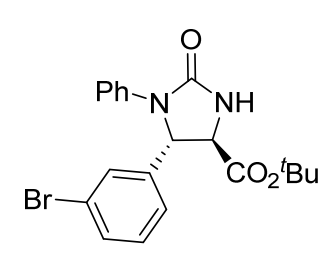

Obtained $77.7 \mathrm{mg}(74 \%)$ from $69.3 \mathrm{mg}(0.25 \mathrm{mmol})$ of $\mathbf{1 g}$. The enantiomeric excess (minor isomer: 9\%, major isomer $89 \%$ ) was determined by HPLC (Amylose 1), hexane:iPrOH 90:10, $1 \mathrm{~mL} \cdot \mathrm{min}^{-1}$, cis-3ga (minor

diastereomer): minor enantiomer, $\mathrm{t}_{\mathrm{r}}=18.3 \mathrm{~min}$, major enantiomer, $\mathrm{t}_{\mathrm{r}}=29.0$ min, trans-(4R,5S)-3ga (major diastereomer): minor enantiomer, $\mathrm{t}_{\mathrm{r}}=20.9 \mathrm{~min}$, major enantiomer, $\mathrm{t}_{\mathrm{r}}=26.9 \mathrm{~min}$.

trans-(4R,5S)-3ga (major diastereomer): white solid, m.p. $163-166^{\circ} \mathrm{C} ;[\alpha]_{\mathrm{D}}{ }^{25}-42.5$ (c 0.84 , $\mathrm{CHCl}_{3}, 89 \%$ ee); ${ }^{1} \mathbf{H}$ NMR $\left(300 \mathrm{MHz}, \mathrm{CDCl}_{3}\right) \delta 7.55(1 \mathrm{H}, \mathrm{t}, J=1.7 \mathrm{~Hz}, \mathrm{Ar}), 7.44-7.38(3 \mathrm{H}, \mathrm{m}$, Ar), $7.31(1 \mathrm{H}, \mathrm{d}, J=7.8 \mathrm{~Hz}, \mathrm{Ar}), 7.24-7.15(3 \mathrm{H}, \mathrm{m}, \mathrm{Ar}), 7.01(1 \mathrm{H}, \mathrm{t}, J=7.4 \mathrm{~Hz}, \mathrm{Ar}), 5.99$ (1H, bs, $\mathrm{NH}), 5.38(1 \mathrm{H}, \mathrm{d}, J=4.2 \mathrm{~Hz}, \mathrm{CH}), 3.95(1 \mathrm{H}, \mathrm{dd}, J=4.3,0.7 \mathrm{~Hz}, \mathrm{CH}), 1.52\left(9 \mathrm{H}, \mathrm{s}, \mathrm{CH}_{3}\right) ;{ }^{13} \mathrm{C}$ NMR (75 MHz, $\left.\mathrm{CDCl}_{3}\right) \delta 169.3(\mathrm{C}), 158.3(\mathrm{C}), 142.0(\mathrm{C}), 138.0(\mathrm{C}), 131.6(\mathrm{CH}), 130.7(\mathrm{CH})$, $129.4(\mathrm{CH}), 128.8(\mathrm{CH}), 124.7(\mathrm{CH}), 123.7(\mathrm{CH}), 123.1(\mathrm{C}), 120.2(\mathrm{CH}), 83.6(\mathrm{C}), 62.4(\mathrm{CH})$, $60.1(\mathrm{CH}), 28.0\left(\mathrm{CH}_{3}\right)$; HRMS (ESI) $\mathrm{m} / \mathrm{z}: 417.0801[\mathrm{M}+\mathrm{H}]^{+}, \mathrm{C}_{20} \mathrm{H}_{22} \mathrm{BrN}_{2} \mathrm{O}_{3}{ }^{+}$requires 417.0808.

cis-3ga (minor diastereomer): white solid, m.p. $204-208{ }^{\circ} \mathrm{C}$; $[\alpha]_{\mathrm{D}}{ }^{25}+6.9\left(\right.$ c $0.77, \mathrm{CHCl}_{3}, 9 \%$ ee); ${ }^{1} \mathbf{H}$ NMR $\left(300 \mathrm{MHz}, \mathrm{CDCl}_{3}\right) \delta 7.48$ (1H, bs, Ar), 7.43-7.33 (3H, m, Ar), 7.28-7.13 (4H, m, Ar), $7.00(1 \mathrm{H}, \mathrm{t}, J=7.3 \mathrm{~Hz}, \mathrm{Ar}), 5.45(1 \mathrm{H}, \mathrm{d}, J=9.6 \mathrm{~Hz}, \mathrm{CH}), 5.30(1 \mathrm{H}, \mathrm{bs}, \mathrm{NH}), 4.70(1 \mathrm{H}, \mathrm{d}, J=9.6$ $\mathrm{Hz}, \mathrm{CH}), 1.12\left(9 \mathrm{H}, \mathrm{s}, \mathrm{CH}_{3}\right) ;{ }^{13} \mathrm{C}$ NMR (75 MHz, $\left.\mathrm{CDCl}_{3}\right) \delta 167.2(\mathrm{C}), 158.3(\mathrm{C}), 138.3$ (C), 137.9 (C), $131.9(\mathrm{CH}), 131.3(\mathrm{CH}), 130.4(\mathrm{CH}), 128.8(\mathrm{CH}), 126.3(\mathrm{CH}), 123.4(\mathrm{CH}), 122.3(\mathrm{C}), 120.5$ $(\mathrm{CH}), 83.0(\mathrm{C}), 61.9(\mathrm{CH}), 57.4(\mathrm{CH}), 27.4\left(\mathrm{CH}_{3}\right)$; HRMS (ESI) m/z: $417.0801[\mathrm{M}+\mathrm{H}]^{+}$, $\mathrm{C}_{20} \mathrm{H}_{22} \mathrm{BrN}_{2} \mathrm{O}_{3}{ }^{+}$requires 417.0808. 


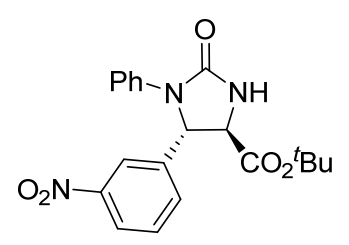

Obtained $88.6 \mathrm{mg}(92 \%)$ from $60.8 \mathrm{mg}(0.25 \mathrm{mmol})$ of $\mathbf{1 h}$. The enantiomeric excess (minor isomer: 27\%, major isomer 83\%) was determined by HPLC (Chiracel IC), hexane:iPrOH 80:20, $1 \mathrm{~mL} \cdot \mathrm{min}^{-1}$, cis-3ha (minor diastereomer): minor enantiomer, $\mathrm{t}_{\mathrm{r}}=39.7 \mathrm{~min}$, major enantiomer, $\mathrm{t}_{\mathrm{r}}=27.5 \mathrm{~min}$, trans-(4R,5S)-3ha (major diastereomer): minor enantiomer, $\mathrm{t}_{\mathrm{r}}=54.5$ min, major enantiomer, $\mathrm{t}_{\mathrm{r}}=57.8 \mathrm{~min}$.

trans-(4R,5S)-3ha (major diastereomer): white solid, m.p. $144-146{ }^{\circ} \mathrm{C} ;[\alpha]_{\mathrm{D}}{ }^{25}-41.6(c 0.84$, $\mathrm{CHCl}_{3}, 83 \%$ ee); ${ }^{1} \mathbf{H}$ NMR $\left(300 \mathrm{MHz}, \mathrm{CDCl}_{3}\right) \delta 8.28(1 \mathrm{H}, \mathrm{t}, J=1.9 \mathrm{~Hz}, \mathrm{Ar}), 8.16(1 \mathrm{H}, \mathrm{ddd}, J=$ 8.2, 2.2, $1.0 \mathrm{~Hz}, \mathrm{Ar}), 7.72(1 \mathrm{H}, \mathrm{d}, J=7.8 \mathrm{~Hz}, \mathrm{Ar}), 7.53(1 \mathrm{H}, \mathrm{t}, J=7.9 \mathrm{~Hz}, \mathrm{Ar}), 7.39$ (2H, dd, $J=$ 8.7, 1.1 Hz, Ar), 7.29-7.20 (2H, m, Ar), $7.03(1 \mathrm{H}, \mathrm{t}, J=7.4 \mathrm{~Hz}, \mathrm{Ar}), 5.70$ (1H, bs, NH), 5.57 (1H, $\mathrm{d}, J=4.6 \mathrm{~Hz}, \mathrm{CH}), 3.98(1 \mathrm{H}, \mathrm{d}, J=4.6 \mathrm{~Hz}, \mathrm{CH}), 1.54\left(9 \mathrm{H}, \mathrm{s}, \mathrm{CH}_{3}\right) ;{ }^{13} \mathbf{C} \mathbf{N M R}\left(75 \mathrm{MHz}, \mathrm{CDCl}_{3}\right) \delta$ 168.9 (C), 157.9 (C), 148.7 (C), 141.8 (C), 137.5 (C), $132.2(\mathrm{CH}), 130.4(\mathrm{CH}), 129.0(\mathrm{CH}), 124.2$ $(\mathrm{CH}), 123.6(\mathrm{CH}), 121.7(\mathrm{CH}), 120.5(\mathrm{CH}), 84.1(\mathrm{C}), 62.3(\mathrm{CH}), 59.8(\mathrm{CH}), 28.0\left(\mathrm{CH}_{3}\right)$; HRMS (ESI) $m / z: 384.1549[\mathrm{M}+\mathrm{H}]^{+}, \mathrm{C}_{20} \mathrm{H}_{22} \mathrm{~N}_{3} \mathrm{O}_{5}{ }^{+}$requires 384.1554 .

cis-3ha (minor diastereomer): white solid, m.p. $187-194{ }^{\circ} \mathrm{C}$; $[\alpha] \mathrm{D}^{25}+0.6\left(\right.$ c $0.38, \mathrm{CHCl}_{3}, 27 \%$ ee); ${ }^{1} \mathbf{H}$ NMR $\left(300 \mathrm{MHz}, \mathrm{CDCl}_{3}\right) \delta 8.22(1 \mathrm{H}, \mathrm{t}, J=2.0 \mathrm{~Hz}, \mathrm{Ar}), 8.14(1 \mathrm{H}, \mathrm{ddd}, J=8.2,2.3,1.0 \mathrm{~Hz}, \mathrm{Ar})$, $7.70(1 \mathrm{H}, \mathrm{d}, J=7.8 \mathrm{~Hz}, \mathrm{Ar}), 7.49(1 \mathrm{H}, \mathrm{t}, J=8.0 \mathrm{~Hz}, \mathrm{Ar}), 7.41-7.31(2 \mathrm{H}, \mathrm{m}, \mathrm{Ar}), 7.22(2 \mathrm{H}, \mathrm{t}, J=$ $8.0 \mathrm{~Hz}, \mathrm{Ar}), 7.01(1 \mathrm{H}, \mathrm{t}, J=7.4 \mathrm{~Hz}, \mathrm{Ar}), 5.64(1 \mathrm{H}, \mathrm{d}, J=9.5 \mathrm{~Hz}, \mathrm{CH}), 5.42(1 \mathrm{H}, \mathrm{bs}, \mathrm{NH}), 4.78(1 \mathrm{H}$, d, $J=9.5 \mathrm{~Hz}, \mathrm{CH}), 1.07\left(9 \mathrm{H}, \mathrm{s}, \mathrm{CH}_{3}\right) ;{ }^{13} \mathrm{C}$ NMR (75 MHz, $\left.\mathrm{CDCl}_{3}\right) \delta 167.9(\mathrm{C}), 158.2(\mathrm{C}), 148.2$ (C), $138.2(\mathrm{C}), 137.5(\mathrm{C}), 133.6(\mathrm{CH}), 130.0(\mathrm{CH}), 129.0(\mathrm{CH}), 124.2(\mathrm{CH}), 123.8(\mathrm{CH}), 123.5$ $(\mathrm{CH}), 120.6(\mathrm{CH}), 83.2(\mathrm{C}), 61.7(\mathrm{CH}), 57.3(\mathrm{CH}), 27.5\left(\mathrm{CH}_{3}\right)$; HRMS (ESI) m/z: 384.1549 $[\mathrm{M}+\mathrm{H}]^{+}, \mathrm{C}_{20} \mathrm{H}_{22} \mathrm{~N}_{3} \mathrm{O}_{5}{ }^{+}$requires 384.1554 .

tert-Butyl (4R,5S)-5-(2-methoxyphenyl)-2-oxo-1-phenylimidazolidine-4-carboxylate (3ia)

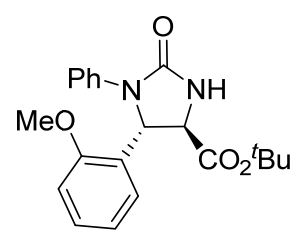

Obtained $77.3 \mathrm{mg}(84 \%)$ from $57.0 \mathrm{mg}(0.25 \mathrm{mmol})$ of $\mathbf{1 i}$. The enantiomeric excess (minor isomer: 73\%, major isomer 94\%) was determined by HPLC (Amylose 1), hexane:iPrOH 90:10, $1 \mathrm{~mL} \cdot \mathrm{min}^{-1}$, cis-3ia (minor diastereomer): minor enantiomer, $\mathrm{t}_{\mathrm{r}}=21.7 \mathrm{~min}$, major enantiomer, $\mathrm{t}_{\mathrm{r}}=48.0$

min, trans-(4R,5S)-3ia (major diastereomer): minor enantiomer, $\mathrm{t}_{\mathrm{r}}=32.5 \mathrm{~min}$, major enantiomer, $\mathrm{t}_{\mathrm{r}}=34.9 \mathrm{~min}$.

trans-(4R,5S)-3ia (major diastereomer): Pale yellow oil; $[\alpha]_{\mathrm{D}}^{25}-70.7$ (c $0.68, \mathrm{CHCl}_{3}, 94 \%$ ee); ${ }^{1} \mathbf{H}$ NMR $\left(300 \mathrm{MHz}, \mathrm{CDCl}_{3}\right) \delta 7.49(2 \mathrm{H}, \mathrm{dd}, J=8.8,1.1 \mathrm{~Hz}, \mathrm{Ar}), 7.35-7.16(4 \mathrm{H}, \mathrm{m}, \mathrm{Ar}), 6.99(1 \mathrm{H}$, 
t, $J=7.4 \mathrm{~Hz}, \mathrm{Ar}), 6.95-6.86(2 \mathrm{H}, \mathrm{m}, \mathrm{Ar}), 5.82(1 \mathrm{H}, \mathrm{d}, J=3.0 \mathrm{~Hz}, \mathrm{CH}), 5.68(1 \mathrm{H}, \mathrm{bs}, \mathrm{NH}), 3.93$ $(1 \mathrm{H}, \mathrm{d}, J=3.0 \mathrm{~Hz}, \mathrm{CH}), 3.92\left(3 \mathrm{H}, \mathrm{s}, \mathrm{CH}_{3}\right), 1.53\left(9 \mathrm{H}, \mathrm{s}, \mathrm{CH}_{3}\right) ;{ }^{13} \mathbf{C} \mathbf{N M R}\left(75 \mathrm{MHz}, \mathrm{CDCl}_{3}\right) \delta 169.9$ (C), $158.7(\mathrm{C}), 156.4(\mathrm{C}), 138.6(\mathrm{C}), 129.3(\mathrm{CH}), 129.0(\mathrm{C}), 128.6(\mathrm{CH}), 128.2(\mathrm{CH}), 126.8(\mathrm{CH})$, $123.0(\mathrm{CH}), 120.8(\mathrm{CH}), 119.3(\mathrm{CH}), 110.65(\mathrm{CH}), 82.5(\mathrm{C}), 59.5(\mathrm{CH}), 57.6(\mathrm{CH}), 55.4\left(\mathrm{CH}_{3}\right)$, $27.9\left(\mathrm{CH}_{3}\right)$; HRMS (ESI) m/z: $369.1807[\mathrm{M}+\mathrm{H}]^{+}, \mathrm{C}_{21} \mathrm{H}_{25} \mathrm{~N}_{2} \mathrm{O}_{4}{ }^{+}$requires 369.1809.

cis-3ia (minor diastereomer): white solid, m.p. 55-59 ${ }^{\circ} \mathrm{C}$; $[\alpha]_{\mathrm{D}}{ }^{25}-17.6\left(\right.$ c $0.26, \mathrm{CHCl}_{3}, 73 \%$ ee); ${ }^{1} \mathbf{H}$ NMR $\left(300 \mathrm{MHz}, \mathrm{CDCl}_{3}\right) \delta 7.37(2 \mathrm{H}, \mathrm{dd}, J=8.7,1.0 \mathrm{~Hz}, \mathrm{Ar}), 7.19(3 \mathrm{H}, \mathrm{t}, J=8.1 \mathrm{~Hz}, \mathrm{Ar}), 7.14$ $(1 \mathrm{H}, \mathrm{dd}, J=7.7,1.6 \mathrm{~Hz}, \mathrm{Ar}), 6.96(1 \mathrm{H}, \mathrm{t}, J=7.4 \mathrm{~Hz}, \mathrm{Ar}), 6.86(1 \mathrm{H}, \mathrm{d}, J=8.2 \mathrm{~Hz}, \mathrm{Ar}), 6.78(1 \mathrm{H}, \mathrm{t}$, $J=7.5 \mathrm{~Hz}, \mathrm{Ar}), 6.10(1 \mathrm{H}, \mathrm{d}, J=9.7 \mathrm{~Hz}, \mathrm{CH}), 5.23(1 \mathrm{H}, \mathrm{bs}, \mathrm{NH}), 4.65(1 \mathrm{H}, \mathrm{d}, J=9.7 \mathrm{~Hz}, \mathrm{CH})$, $3.93\left(3 \mathrm{H}, \mathrm{s}, \mathrm{CH}_{3}\right), 1.02\left(9 \mathrm{H}, \mathrm{s}, \mathrm{CH}_{3}\right) ;{ }^{13} \mathrm{C}$ NMR $\left(75 \mathrm{MHz}, \mathrm{CDCl}_{3}\right) \delta 168.4(\mathrm{C}), 159.2(\mathrm{C}), 157.4$ (C), $138.4(\mathrm{C}), 129.4(\mathrm{CH}), 128.5(\mathrm{CH}), 128.0(\mathrm{CH}), 123.8(\mathrm{C}), 123.3(\mathrm{CH}), 120.9(\mathrm{CH}), 120.2$ $\left.(\mathrm{CH}), 109.9(\mathrm{CH}), 82.0(\mathrm{C}), 56.3(\mathrm{CH}), 55.4\left(\mathrm{CH}_{3}\right), 54.9 \mathrm{CH}\right), 27.3\left(\mathrm{CH}_{3}\right)$; HRMS (ESI) m/z: $369.1807[\mathrm{M}+\mathrm{H}]^{+}, \mathrm{C}_{21} \mathrm{H}_{25} \mathrm{~N}_{2} \mathrm{O}_{4}{ }^{+}$requires 369.1809 .

tert-Butyl (4R,5S)-5-(2-bromophenyl)-2-oxo-1-phenylimidazolidine-4-carboxylate (3ja)

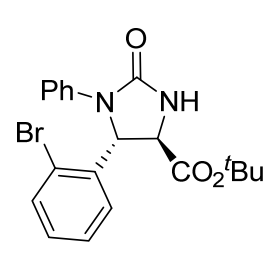

Obtained $86.8 \mathrm{mg}(83 \%)$ from $69.3 \mathrm{mg}(0.25 \mathrm{mmol})$ of $\mathbf{1 j}$. The enantiomeric excess (minor isomer: 86\%, major isomer 87\%) was determined by HPLC (Amylose 1), hexane:iPrOH 80:20, $1 \mathrm{~mL} \cdot \mathrm{min}^{-1}$, cis-3ja (minor diastereomer): minor enantiomer, $\mathrm{t}_{\mathrm{r}}=10.3 \mathrm{~min}$, major enantiomer, $\mathrm{t}_{\mathrm{r}}=16.6 \mathrm{~min}$, trans-

$(\mathbf{A R}, \mathbf{5 S})$-3ja (major diastereomer): minor enantiomer, $\mathrm{t}_{\mathrm{r}}=12.9 \mathrm{~min}$, major enantiomer, $\mathrm{t}_{\mathrm{r}}=16.9$ min.

trans-(4R,5S)-3ja (major diastereomer): brown oil; $[\alpha]_{\mathrm{D}}{ }^{25}-93.2\left(\right.$ c $0.77, \mathrm{CHCl}_{3}, 87 \%$ ee); ${ }^{1} \mathbf{H}$ NMR $\left(300 \mathrm{MHz}, \mathrm{CDCl}_{3}\right) \delta 7.60(1 \mathrm{H}, \mathrm{dd}, J=7.9,1.2 \mathrm{~Hz}, \mathrm{Ar}), 7.44(2 \mathrm{H}, \mathrm{dd}, J=8.7,1.0 \mathrm{~Hz}, \mathrm{Ar})$, $7.34(1 \mathrm{H}, \mathrm{dd}, J=7.8,1.8 \mathrm{~Hz}, \mathrm{Ar}), 7.29-7.20$ (3H, m, Ar), 7.16 (1H, dt, $J=7.9,1.9 \mathrm{~Hz}, \mathrm{Ar}), 6.99$ $(1 \mathrm{H}, \mathrm{t}, J=7.4 \mathrm{~Hz}, \mathrm{Ar}), 5.95(1 \mathrm{H}, \mathrm{bs}, \mathrm{NH}), 5.87(1 \mathrm{H}, \mathrm{d}, J=2.3 \mathrm{~Hz}, \mathrm{CH}), 3.92(1 \mathrm{H}, \mathrm{bs}, \mathrm{CH}), 1.52$ (9H, s, $\left.\mathrm{CH}_{3}\right) ;{ }^{13} \mathrm{C}$ NMR (75 MHz, $\left.\mathrm{CDCl}_{3}\right) \delta 169.2$ (C), 158.7 (C), 138.1 (C), 137.7 (C), 133.5 $(\mathrm{CH}), 129.9(\mathrm{CH}), 128.8(\mathrm{CH}), 128.2(\mathrm{CH}), 127.6(\mathrm{CH}), 123.4(\mathrm{CH}), 122.32(\mathrm{C}), 119.4(\mathrm{CH}), 83.3$ (C), $61.8(\mathrm{CH}), 59.4(\mathrm{CH}), 27.9\left(\mathrm{CH}_{3}\right)$; HRMS (ESI) m/z: $417.0801[\mathrm{M}+\mathrm{H}]^{+}, \mathrm{C}_{20} \mathrm{H}_{22} \mathrm{BrN}_{2} \mathrm{O}_{3}{ }^{+}$ requires 417.0808 .

cis-3ja (minor diastereomer): brown oil; $[\alpha]_{\mathrm{D}^{25}}-11.2$ (c $0.56, \mathrm{CHCl}_{3}, 86 \%$ ee); ${ }^{1} \mathbf{H}$ NMR (300 $\left.\mathrm{MHz}, \mathrm{CDCl}_{3}\right) \delta 7.54(1 \mathrm{H}, \mathrm{dd}, J=7.7,1.5 \mathrm{~Hz}, \mathrm{Ar}), 7.34(2 \mathrm{H}, \mathrm{dd}, J=8.7,1.1 \mathrm{~Hz}, \mathrm{Ar}), 7.29(1 \mathrm{H}, \mathrm{dd}$, $J=7.6,1.9 \mathrm{~Hz}, \mathrm{Ar}), 7.24-7.18(2 \mathrm{H}, \mathrm{m}, \mathrm{Ar}), 7.15(1 \mathrm{H}, \mathrm{dd}, J=7.6,1.5 \mathrm{~Hz}, \mathrm{Ar}), 7.10(1 \mathrm{H}, \mathrm{dt}, J=7.7$, $1.9 \mathrm{~Hz}, \mathrm{Ar}), 6.99(1 \mathrm{H}, \mathrm{t}, J=7.3 \mathrm{~Hz}, \mathrm{Ar}), 6.10(1 \mathrm{H}, \mathrm{d}, J=10.0 \mathrm{~Hz}, \mathrm{CH}), 5.32(1 \mathrm{H}, \mathrm{bs}, \mathrm{NH}), 4.74$ $(1 \mathrm{H}, \mathrm{d}, J=10.0 \mathrm{~Hz}, \mathrm{CH}), 1.06\left(9 \mathrm{H}, \mathrm{s}, \mathrm{CH}_{3}\right) ;{ }^{13} \mathrm{C} \mathrm{NMR}\left(75 \mathrm{MHz}, \mathrm{CDCl}_{3}\right) \delta 167.9$ (C), $158.7(\mathrm{C})$, 
$137.8(\mathrm{C}), 135.1(\mathrm{C}), 132.6(\mathrm{CH}), 130.0(\mathrm{CH}), 129.1(\mathrm{CH}), 128.7(\mathrm{CH}), 128.1(\mathrm{CH}), 124.7(\mathrm{C})$, $123.8(\mathrm{CH}), 120.4(\mathrm{CH}), 82.8(\mathrm{C}), 60.6(\mathrm{CH}), 55.8(\mathrm{CH}), 27.4\left(\mathrm{CH}_{3}\right)$; HRMS (ESI) m/z: 417.0801 $[\mathrm{M}+\mathrm{H}]^{+}, \mathrm{C}_{20} \mathrm{H}_{22} \mathrm{BrN}_{2} \mathrm{O}_{3}{ }^{+}$requires 417.0808 .

\section{tert-Butyl (4R,5S)-5-(2-nitrophenyl)-2-oxo-1-phenylimidazolidine-4-carboxylate (3ka)}

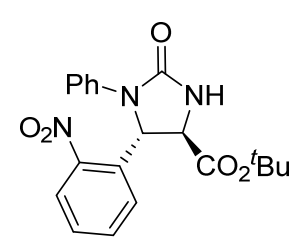

Obtained $87.3 \mathrm{mg}(91 \%)$ from $60.8 \mathrm{mg}(0.25 \mathrm{mmol})$ of $\mathbf{1 k}$. The enantiomeric excess (minor isomer: 70\%, major isomer 67\%) was determined by HPLC (Chiracel IC), hexane:iPrOH 80:20, $1 \mathrm{~mL} \cdot \mathrm{min}^{-1}$, cis-3ka (minor diastereomer): minor enantiomer, $\mathrm{t}_{\mathrm{r}}=61.8 \mathrm{~min}$, major enantiomer, $\mathrm{t}_{\mathrm{r}}=66.0$ min, trans-(4R,5S)-3ka (major diastereomer): minor enantiomer, $t_{r}=18.0 \mathrm{~min}$, major enantiomer, $\mathrm{t}_{\mathrm{r}}=20.8 \mathrm{~min}$. Both diastereomers could not be separated by column chromatography.

trans-(4R,5S)-3ka (major diastereomer): yellow oil; $[\alpha]_{\mathrm{D}}{ }^{25}-49.2\left(\right.$ c $0.57, \mathrm{CHCl}_{3}$, for the diastereomer mixture, dr: 64:36); ${ }^{1} \mathbf{H}$ NMR $\left(300 \mathrm{MHz}, \mathrm{CDCl}_{3}\right) \delta 8.07(1 \mathrm{H}, \mathrm{dd}, J=8.1,1.0 \mathrm{~Hz}, \mathrm{Ar})$, 7.65-7.40 (4H, m, Ar), 7.28-7.22 (2H, m, Ar), 7.19-7.13 (1H, m, Ar), 7.02 (1H, t, J = 7.4 Hz, Ar), $6.20(1 \mathrm{H}, \mathrm{d}, J=2.4 \mathrm{~Hz}, \mathrm{CH}), 5.55(1 \mathrm{H}, \mathrm{bs}, \mathrm{NH}), 3.94(1 \mathrm{H}, \mathrm{d}, J=3.94, \mathrm{CH}), 1.53\left(9 \mathrm{H}, \mathrm{s}, \mathrm{CH}_{3}\right) ;{ }^{13} \mathrm{C}$ NMR (75 MHz, $\left.\mathrm{CDCl}_{3}\right) \delta 169.0$ (C), 158.4 (C), 147.8 (C), 137.9 (C), 134.2 (CH), 134.1 (C), $129.4(\mathrm{CH}), 129.0(\mathrm{CH}), 128.9(\mathrm{CH}), 128.2(\mathrm{CH}), 127.8(\mathrm{CH}), 125.6(\mathrm{CH}), 123.8(\mathrm{CH}), 119.5$ $(\mathrm{CH}), 83.6(\mathrm{C}), 59.6(\mathrm{CH}), 58.4(\mathrm{CH}), 27.9\left(\mathrm{CH}_{3}\right)$; HRMS (ESI) m/z: $384.1549[\mathrm{M}+\mathrm{H}]^{+}$, $\mathrm{C}_{20} \mathrm{H}_{22} \mathrm{~N}_{3} \mathrm{O}_{5}^{+}$requires 384.1554 .

cis-3ka (minor diastereomer): ${ }^{1} \mathbf{H}$ NMR (300 $\left.\mathrm{MHz}, \mathrm{CDCl}_{3}\right)$, significative signals taken from the NMR spectrum, of the diastereomer mixture, $\delta 8.13(1 \mathrm{H}, \mathrm{dd}, J=7.9,1.3 \mathrm{~Hz}, \mathrm{Ar}), 7.65-7.40(3 \mathrm{H}$, m, Ar), 7.33 (2H, dd, $J=8.7,1.1 \mathrm{~Hz}, \mathrm{Ar}), 7.23-7.22$ (1H, m, Ar), 7.19-7.13 (1H, m, Ar), 7.05 (1H, m, Ar), $6.57(1 \mathrm{H}, \mathrm{d}, J=10.0 \mathrm{~Hz}, \mathrm{CH}), 5.46(1 \mathrm{H}, \mathrm{bs}, \mathrm{NH}), 4.88(1 \mathrm{H}, \mathrm{d}, J=10.0 \mathrm{~Hz}, \mathrm{Ar}), 1.01(9 \mathrm{H}$, s, $\left.\mathrm{CH}_{3}\right) ;{ }^{13} \mathrm{C}$ NMR (75 MHz, $\left.\mathrm{CDCl}_{3}\right) \delta 168.3(\mathrm{C}), 158.9(\mathrm{CH}), 149.0(\mathrm{C}), 137.6(\mathrm{C}), 134.4(\mathrm{CH})$, $131.8(\mathrm{C}), 129.6(\mathrm{CH}), 129.4(\mathrm{CH}), 125.3(\mathrm{CH}), 125.2(\mathrm{CH}), 124.5(\mathrm{CH}), 120.9(\mathrm{CH}), 82.9(\mathrm{C})$, $57.2(\mathrm{CH}), 56.1(\mathrm{CH}), 27.3\left(\mathrm{CH}_{3}\right)$.

\section{tert-Butyl (4R,5S)-5-(naphthalen-1-yl)-2-oxo-1-phenylimidazolidine-4-carboxylate (3la)}

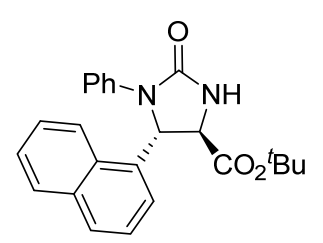

Obtained $73.4 \mathrm{mg}(75 \%)$ from $62.1 \mathrm{mg}(0.25 \mathrm{mmol})$ of $\mathbf{1 1}$. The enantiomeric excess (minor isomer: 68\%, major isomer 94\%) was determined by HPLC (Chiracel IC), hexane:iPrOH 80:20, $1 \mathrm{~mL} \cdot \mathrm{min}^{-1}$, cis-3la (minor diastereomer): minor enantiomer, $\mathrm{t}_{\mathrm{r}}=33.0 \mathrm{~min}$, major enantiomer, $\mathrm{t}_{\mathrm{r}}=46.1$

min, trans-(4R,5S)-3la (major diastereomer): minor enantiomer, $\mathrm{t}_{\mathrm{r}}=19.6 \mathrm{~min}$, major enantiomer, $\mathrm{t}_{\mathrm{r}}=22.8 \mathrm{~min}$. 
trans-(4R,5S)-3la (major diastereomer): grey oil; $[\alpha]_{\mathrm{D}}{ }^{25}-96.0$ (c $0.81, \mathrm{CHCl}_{3}, 94 \%$ ee); ${ }^{1} \mathbf{H}$ NMR $\left(300 \mathrm{MHz}, \mathrm{CDCl}_{3}\right) \delta 8.44(1 \mathrm{H}, \mathrm{d}, J=8.3 \mathrm{~Hz}, \mathrm{Ar}), 7.95(1 \mathrm{H}, \mathrm{d}, J=7.7 \mathrm{~Hz}, \mathrm{Ar}), 7.83(1 \mathrm{H}, \mathrm{d}$, $J=8.0 \mathrm{~Hz}, \mathrm{Ar}), 7.65(1 \mathrm{H}, \mathrm{dt}, J=6.9,1.5 \mathrm{~Hz}, \mathrm{Ar}), 7.58(1 \mathrm{H}, \mathrm{t}, J=6.9 \mathrm{~Hz}, \mathrm{Ar}), 7.50(2 \mathrm{H}, \mathrm{d}, J=8.1$ $\mathrm{Hz}, \mathrm{Ar}), 7.46$ (1, d, $J=7.4 \mathrm{~Hz}, \mathrm{Ar}), 7.43-7.36(1 \mathrm{H}, \mathrm{m}, \mathrm{Ar}), 7.19$ (2H, t, $J=8.0 \mathrm{~Hz}, \mathrm{Ar}), 6.97$ (1H, t, $J=7.4 \mathrm{~Hz}, \mathrm{Ar}), 6.24(1 \mathrm{H}, \mathrm{bs}, \mathrm{CH}), 5.73(1 \mathrm{H}, \mathrm{bs}, \mathrm{NH}), 3.97(1 \mathrm{H}, \mathrm{bs}, \mathrm{CH}), 1.60\left(9 \mathrm{H}, \mathrm{s}, \mathrm{CH}_{3}\right) ;{ }^{13} \mathrm{C}$ NMR (75 MHz, $\left.\mathrm{CDCl}_{3}\right) \delta 170.0(\mathrm{C}), 158.6(\mathrm{C}), 138.6(\mathrm{C}), 134.3(\mathrm{CH}), 133.4(\mathrm{CH}), 129.9(\mathrm{C})$, $129.3(\mathrm{CH}), 128.9(\mathrm{C}), 128.7(\mathrm{CH}), 126.7(\mathrm{CH}), 125.9(\mathrm{CH}), 125.5(\mathrm{CH}), 123.0(\mathrm{C}), 122.5(\mathrm{CH})$, $118.8(\mathrm{CH}), 83.7(\mathrm{C}), 59.52(\mathrm{CH}), 59.50(\mathrm{CH}), 28.0\left(\mathrm{CH}_{3}\right)$; HRMS (ESI) m/z: $389.1841[\mathrm{M}+\mathrm{H}]^{+}$, $\mathrm{C}_{24} \mathrm{H}_{25} \mathrm{~N}_{2} \mathrm{O}_{3}{ }^{+}$requires 389.1860 .

cis-3la (minor diastereomer): colorless oil; $[\alpha]]^{25}-2.4$ (c $0.60, \mathrm{CHCl}_{3}, 68 \%$ ee); ${ }^{1} \mathbf{H}$ NMR (300 $\left.\mathrm{MHz}_{\mathrm{CDCl}}\right) \delta 8.17(1 \mathrm{H}, \mathrm{d}, J=8.4 \mathrm{~Hz}, \mathrm{Ar}), 7.89(1 \mathrm{H}, \mathrm{d}, J=8.1 \mathrm{~Hz}, \mathrm{Ar}), 7.75(1 \mathrm{H}, \mathrm{d}, J=8.1 \mathrm{~Hz}$, Ar), 7.68-7.60 (1H, m, Ar), 7.58-7.51 (1H, m, Ar), 7.45 (1H, dd, J= 7.3, 0.9 Hz, Ar), 7.36-7.29 (3H, m, Ar), 7.18-7.08 (2H, m, Ar), $6.93(1 \mathrm{H}, \mathrm{t}, J=7.4 \mathrm{~Hz}, \mathrm{Ar}), 6.47(1 \mathrm{H}, \mathrm{d}, J=9.9 \mathrm{~Hz}, \mathrm{CH}), 5.36$ $(1 \mathrm{H}, \mathrm{bs}, \mathrm{NH}), 4.86(1 \mathrm{H}, \mathrm{d}, J=9.9 \mathrm{~Hz}, \mathrm{CH}), 0.60\left(9 \mathrm{H}, \mathrm{s}, \mathrm{CH}_{3}\right) ;{ }^{13} \mathbf{C} \mathbf{N M R}\left(75 \mathrm{MHz}, \mathrm{CDCl}_{3}\right) \delta$ 168.0 (C), 159.1 (C), 138.4 (C), 133.6 (C), 131.7 (C), 131.0 (C), $129.1(\mathrm{CH}), 128.9$ (CH), 128.6 $(\mathrm{CH}), 126.7(\mathrm{CH}), 125.8(\mathrm{CH}), 125.7(\mathrm{CH}), 125.4(\mathrm{CH}), 123.4(\mathrm{CH}), 122.6(\mathrm{CH}), 120.1(\mathrm{CH})$, $82.2(\mathrm{C}), 57.4(\mathrm{CH}), 56.9(\mathrm{CH}), 26.8\left(\mathrm{CH}_{3}\right)$; HRMS (ESI) $\mathrm{m} / \mathrm{z}: 389.1841[\mathrm{M}+\mathrm{H}]^{+}, \mathrm{C}_{24} \mathrm{H}_{25} \mathrm{~N}_{2} \mathrm{O}_{3}{ }^{+}$ requires 389.1860 .

\section{tert-Butyl (4R,5R)-2-oxo-1-phenyl-5-(pyridin-2-yl)imidazolidine-4-carboxylate (3ma)}

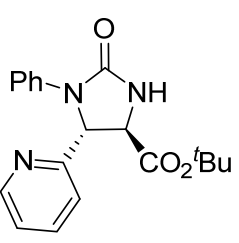

Obtained $84.2 \mathrm{mg}(99 \%)$ from $49.8 \mathrm{mg}(0.25 \mathrm{mmol})$ of $\mathbf{1 m}$. The enantiomeric excess (minor isomer: 7\%, major isomer 95\%) was determined by HPLC (Amylose 1), hexane:iPrOH 80:20, $1 \mathrm{~mL} \cdot \mathrm{min}^{-1}$, cis-3ma (minor diastereomer): minor enantiomer, $\mathrm{t}_{\mathrm{r}}=28.8 \mathrm{~min}$, major enantiomer, $\mathrm{t}_{\mathrm{r}}=25.0$ min, trans-(4R,5S)-3ma (major diastereomer): minor enantiomer, $t_{\mathrm{r}}=16.4 \mathrm{~min}$, major enantiomer, $\mathrm{t}_{\mathrm{r}}=30.4 \mathrm{~min}$. Both diastereomers could not be separated by column chromatography.

trans-(4R,5S)-3ma (major diastereomer): yellow oil; $[\alpha]_{\mathrm{D}}^{25}-50.1$ (c $0.96, \mathrm{CHCl}_{3}$, for the diastereomer mixture, dr: 88:12); ${ }^{1} \mathbf{H}$ NMR $\left(300 \mathrm{MHz}, \mathrm{CDCl}_{3}\right) \delta 8.65(1 \mathrm{H}$, ddd, $J=4.8,1.7,0.9$ $\mathrm{Hz}, \mathrm{Ar}), 7.63(1 \mathrm{H}, \mathrm{td}, J=7.7,1.8 \mathrm{~Hz}, \mathrm{Ar}), 7.50(2 \mathrm{H}, \mathrm{dd}, J=8.7,1.0 \mathrm{~Hz}, \mathrm{Ar}), 7.32-7.18(4 \mathrm{H}, \mathrm{m}$, Ar), $7.01(1 \mathrm{H}, \mathrm{t}, J=7.4 \mathrm{~Hz}, \mathrm{Ar}), 6.17(1 \mathrm{H}, \mathrm{bs}, \mathrm{NH}), 5.60(1 \mathrm{H}, \mathrm{d}, J=3.5 \mathrm{~Hz}, \mathrm{CH}), 4.19(1 \mathrm{H}, \mathrm{dd}, J$ $=3.5,0.7 \mathrm{~Hz}, \mathrm{CH}), 1.52\left(9 \mathrm{H}, \mathrm{s}, \mathrm{CH}_{3}\right) ;{ }^{13} \mathrm{C}$ NMR (75 MHz, $\left.\mathrm{CDCl}_{3}\right) \delta 169.4(\mathrm{C}), 158.44(\mathrm{C})$, $158.37(\mathrm{C}), 150.0(\mathrm{CH}), 138.3(\mathrm{C}), 137.0(\mathrm{CH}), 128.7(\mathrm{CH}), 123.3(\mathrm{CH}), 123.1(\mathrm{CH}), 120.9(\mathrm{CH})$, $119.7(\mathrm{CH}), 83.1(\mathrm{C}), 64.1(\mathrm{CH}), 58.7(\mathrm{CH}), 27.8\left(\mathrm{CH}_{3}\right)$; HRMS (ESI) m/z: $340.1657[\mathrm{M}+\mathrm{H}]^{+}$, $\mathrm{C}_{19} \mathrm{H}_{22} \mathrm{~N}_{3} \mathrm{O}_{3}{ }^{+}$requires 340.1656 . 
cis-3ma (minor diastereomer): ${ }^{1} \mathbf{H}$ NMR (300 $\mathrm{MHz}, \mathrm{CDCl}_{3}$ ), signals taken from the NMR spectrum, of the diastereomer mixture, $\delta 8.60(1 \mathrm{H}, \mathrm{dd}, J=4.8,0.9 \mathrm{~Hz}, \mathrm{Ar}), 7.56(1 \mathrm{H}, \mathrm{dd}, J=6.2$, $1.5 \mathrm{~Hz}, \mathrm{Ar}), 7.44$ (2H, d, J=7.9 Hz, Ar), 7.38-7.18 (4H, m, Ar), 6.98 (1H, t, J 7.4 Hz, Ar), 6.07 $(1 \mathrm{H}, \mathrm{bs}, \mathrm{NH}), 5.75(1 \mathrm{H}, \mathrm{dd}, J=9.9,3.3 \mathrm{~Hz}, \mathrm{CH}), 4.82(1 \mathrm{H}, \mathrm{ddd}, J=9.9,1.6,0.8 \mathrm{~Hz}, \mathrm{CH}), 1.1(9 \mathrm{H}$, $\left.\mathrm{d}, J=1.8 \mathrm{~Hz}, \mathrm{CH}_{3}\right) ;{ }^{13} \mathrm{C}$ NMR $\left(75 \mathrm{MHz}, \mathrm{CDCl}_{3}\right) \delta 167.7(\mathrm{C}), 158.8(\mathrm{C}), 156.3(\mathrm{C}), 149.1(\mathrm{CH})$, $138.1(\mathrm{C}), 136.9(\mathrm{CH}), 128.5(\mathrm{CH}), 125.5(\mathrm{CH}), 122.3(\mathrm{CH}), 120.1(\mathrm{CH}), 82.4(\mathrm{C}), 63.8(\mathrm{CH})$, $56.6(\mathrm{CH}), 27.3\left(\mathrm{CH}_{3}\right)$.

ent-3ma: Obtained $83.1 \mathrm{mg}$ (98\%) from $49.8 \mathrm{mg}(0.25 \mathrm{mmol})$ of 1m, using SQ7 as organocatalyst. The enantiomeric excess (minor isomer: 22\%, major isomer 92\%) was determined by HPLC (Amylose 1), hexane:iPrOH 80:20, $1 \mathrm{~mL} \cdot \mathrm{min}^{-1}$, cis-3ma (minor diastereomer): minor enantiomer, $t_{r}=23.9$ min, major enantiomer, $t_{r}=26.3 \mathrm{~min}$, trans-(4R,5S)-3ma (major diastereomer): minor enantiomer, $\mathrm{t}_{\mathrm{r}}=29.7 \mathrm{~min}$, major enantiomer, $\mathrm{t}_{\mathrm{r}}=15.7 \mathrm{~min} . \quad[\alpha]_{\mathrm{D}}{ }^{25}+45.1(\mathrm{c}$ $0.97, \mathrm{CHCl}_{3}$, for the diastereomer mixture, $\left.\mathrm{dr}: 86: 14\right)$;

tert-Butyl (4R,5S)-5-cyclohexyl-2-oxo-1-phenylimidazolidine-4-carboxylate (3na)

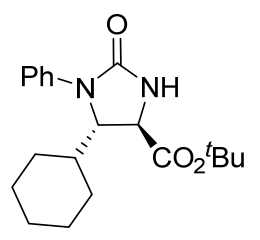

Obtained $58.3 \mathrm{mg}(67 \%)$ from $51.0 \mathrm{mg}(0.25 \mathrm{mmol})$ of $\mathbf{1 n}$. The enantiomeric excess (minor isomer: nd, major isomer 91\%) was determined by HPLC (Amylose 1), hexane:iPrOH 90:10, $1 \mathrm{~mL} \cdot \mathrm{min}^{-1}$, trans-(4R,5S)-3na (major diastereomer): minor enantiomer, $\mathrm{t}_{\mathrm{r}}=13.5 \mathrm{~min}$, major enantiomer, $\mathrm{t}_{\mathrm{r}}=15.9$ $\min$.

trans-(4R,5S)-3na (major diastereomer): white solid, m.p. $180-187{ }^{\circ} \mathrm{C} ;[\alpha]_{\mathrm{D}}{ }^{25}-34.9(\mathrm{c} 0.95$, $\mathrm{CHCl}_{3}, 91 \%$ ee); ${ }^{1} \mathbf{H}$ NMR $\left(300 \mathrm{MHz}, \mathrm{CDCl}_{3}\right) \delta 7.42(2 \mathrm{H}, \mathrm{dd}, J=8.7,1.2 \mathrm{~Hz}, \mathrm{Ar}), 7.38-7.30(2 \mathrm{H}$, m, Ar), $7.10(1 \mathrm{H}, \mathrm{t}, J=7.3 \mathrm{~Hz}, \mathrm{Ar}), 5.59(1 \mathrm{H}, \mathrm{bs}, \mathrm{NH}), 4.39(1 \mathrm{H}, \mathrm{t}, J=3.3 \mathrm{~Hz}, \mathrm{CH}), 3.92(1 \mathrm{H}, \mathrm{d}, J$ $=3.1 \mathrm{~Hz}, \mathrm{CH}), 1.80-1.57(6 \mathrm{H}, \mathrm{m}), 1.47\left(9 \mathrm{H}, \mathrm{s}, \mathrm{CH}_{3}\right), 1.26-0.90(5 \mathrm{H}, \mathrm{m}) ;{ }^{13} \mathrm{C}$ NMR $(75 \mathrm{MHz}$, $\left.\mathrm{CDCl}_{3}\right) \delta 171.0(\mathrm{C}), 158.4(\mathrm{C}), 137.8(\mathrm{C}), 128.9(\mathrm{CH}), 124.1(\mathrm{CH}), 122.0(\mathrm{CH}), 82.7(\mathrm{C}), 63.8$ $(\mathrm{CH}), 53.1(\mathrm{CH}), 38.7(\mathrm{CH}), 28.4\left(\mathrm{CH}_{2}\right), 27.9\left(\mathrm{CH}_{3}\right), 26.3\left(\mathrm{CH}_{2}\right), 26.0\left(\mathrm{CH}_{2}\right), 25.5\left(\mathrm{CH}_{2}\right), 25.4$ $\left(\mathrm{CH}_{2}\right)$; HRMS (ESI) m/z: $345.2161[\mathrm{M}+\mathrm{H}]^{+}, \mathrm{C}_{20} \mathrm{H}_{29} \mathrm{~N}_{2} \mathrm{O}_{3}{ }^{+}$requires 345.2173.

tert-Butyl (4R,5S)-5-cyclopropyl-2-oxo-1-phenylimidazolidine-4-carboxylate (3oa)

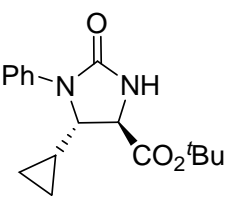

Obtained $72.9 \mathrm{mg}(96 \%)$ from $40.4 \mathrm{mg}(0.25 \mathrm{mmol})$ of $1 \mathbf{1 0}$. The enantiomeric excess (minor isomer: 68\%, major isomer 99\%) was determined by HPLC (Amylose 1), hexane: $\mathrm{PrOH} 80: 20,1 \mathrm{~mL} \cdot \mathrm{min}^{-1}$, cis-3oa (minor diastereomer): minor enantiomer, $\mathrm{t}_{\mathrm{r}}=9.8 \mathrm{~min}$, major enantiomer, $\mathrm{t}_{\mathrm{r}}=20.7 \mathrm{~min}$, trans-(4R,5S)-3oa (major diastereomer): minor enantiomer, $\mathrm{t}_{\mathrm{r}}=11.4 \mathrm{~min}$, major enantiomer, $\mathrm{t}_{\mathrm{r}}=18.3 \mathrm{~min}$. 
trans-(4R,5S)-3oa (major diastereomer): white solid, m.p. $142-145^{\circ} \mathrm{C} ;[\alpha]_{\mathrm{D}}{ }^{25}-64.5(\mathrm{c} 0.79$, $\mathrm{CHCl}_{3}, 99 \%$ ee); ${ }^{1} \mathbf{H}$ NMR $\left(300 \mathrm{MHz}, \mathrm{CDCl}_{3}\right) \delta 7.38(2 \mathrm{H}, \mathrm{dd}, J=8.6,1.4 \mathrm{~Hz}, \mathrm{Ar}), 7.36-7.29(2 \mathrm{H}$, m, Ar), $7.14(1 \mathrm{H}, \mathrm{t}, J=7.0 \mathrm{~Hz}, \mathrm{Ar}), 5.8(1 \mathrm{H}, \mathrm{bs}, \mathrm{NH}), 3.99(1 \mathrm{H}, \mathrm{d}, J=3.5 \mathrm{~Hz}, \mathrm{CH}), 3.75(1 \mathrm{H}, \mathrm{dd}$, $J=8.7,3.5 \mathrm{~Hz}, \mathrm{CH}), 1.46\left(9 \mathrm{H}, \mathrm{s}, \mathrm{CH}_{3}\right), 1.21-1.05(1 \mathrm{H}, \mathrm{m}, \mathrm{CH}), 0.56-0.40\left(2 \mathrm{H}, \mathrm{m}, \mathrm{CH}_{2}\right), 0.35-0.17$ $\left(2 \mathrm{H}, \mathrm{m}, \mathrm{CH}_{2}\right) ;{ }^{13} \mathrm{C}$ NMR (75 MHz, $\left.\mathrm{CDCl}_{3}\right) \delta 170.3(\mathrm{C}), 158.5(\mathrm{C}), 138.1(\mathrm{C}), 128.8(\mathrm{CH}), 125.0$ $(\mathrm{CH}), 124.0(\mathrm{CH}), 82.7(\mathrm{C}), 64.9(\mathrm{CH}), 58.3(\mathrm{CH}), 27.8\left(\mathrm{CH}_{3}\right), 15.6(\mathrm{CH}), 5.47\left(\mathrm{CH}_{2}\right), 0.7\left(\mathrm{CH}_{2}\right)$; HRMS (ESI) m/z: $303.1693[\mathrm{M}+\mathrm{H}]^{+}, \mathrm{C}_{17} \mathrm{H}_{23} \mathrm{~N}_{2} \mathrm{O}_{3}{ }^{+}$requires 303.1703.

cis-3oa (minor diastereomer): white solid, m.p. $177-180{ }^{\circ} \mathrm{C} ;[\alpha]_{\mathrm{D}}{ }^{25}+17.9\left(c 0.81, \mathrm{CHCl}_{3}, 68 \%\right.$ ee); ${ }^{1} \mathbf{H}$ NMR (300 MHz, $\left.\mathrm{CDCl}_{3}\right) \delta$ 7.40-7.30 (4H, m, Ar), 7.19 (1H, ddd, J=8.6, 5.7, $2.5 \mathrm{~Hz}, \mathrm{Ar}$ ), $5.15(1 \mathrm{H}, \mathrm{bs}, \mathrm{NH}), 4.41(1 \mathrm{H}, \mathrm{d}, J=8.6 \mathrm{~Hz}, \mathrm{CH}), 3.75-3.67(1 \mathrm{H}, \mathrm{m}, \mathrm{CH}), 1.52\left(9 \mathrm{H}, \mathrm{s}, \mathrm{CH}_{3}\right), 1.03-$ $0.89(1 \mathrm{H}, \mathrm{m}, \mathrm{CH}), 0.52-0.31\left(2 \mathrm{H}, \mathrm{m}, \mathrm{CH}_{2}\right), 0.23(1 \mathrm{H}, \mathrm{td}, J=9.7,5.0 \mathrm{~Hz}, \mathrm{CH}),-0.01(1 \mathrm{H}, \mathrm{td}, J=$ 9.8, $4.8 \mathrm{~Hz}, \mathrm{CH}) ;{ }^{13} \mathrm{C}$ NMR (75 MHz, $\left.\mathrm{CDCl}_{3}\right) \delta 168.7$ (C), 159.3 (C), 138.0 (C), $128.9(\mathrm{CH})$, $125.7(\mathrm{CH}), 125.6(\mathrm{CH}), 82.6(\mathrm{C}), 65.0(\mathrm{CH}), 57.6(\mathrm{CH}), 28.0\left(\mathrm{CH}_{3}\right), 10.8(\mathrm{CH}), 4.8\left(\mathrm{CH}_{2}\right), 2.5$ $\left(\mathrm{CH}_{2}\right)$; HRMS (ESI) m/z: $303.1693[\mathrm{M}+\mathrm{H}]^{+}, \mathrm{C}_{17} \mathrm{H}_{23} \mathrm{~N}_{2} \mathrm{O}_{3}{ }^{+}$requires 303.1703.

\section{tert-Butyl (4R,5S)-1-(4-chlorophenyl)-2-oxo-5-phenylimidazolidine-4-carboxylate (3pa)}

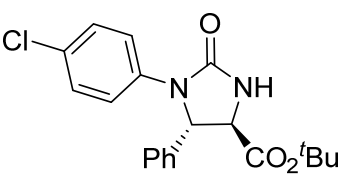

Obtained $77.5 \mathrm{mg}(83 \%)$ from $58.2 \mathrm{mg}(0.25 \mathrm{mmol})$ of 1p. The enantiomeric excess (minor isomer: 6\%, major isomer 84\%) was determined by HPLC (Amylose 1), hexane:iPrOH 80:20, $1 \mathrm{~mL} \cdot \mathrm{min}^{-1}$, cis-

3pa (minor diastereomer): minor enantiomer, $t_{r}=9.4$ min, major enantiomer, $t_{r}=18.8$ min, trans-(4R,5S)-3pa (major diastereomer): minor enantiomer, $\mathrm{t}_{\mathrm{r}}=20.2 \mathrm{~min}$, major enantiomer, $\mathrm{t}_{\mathrm{r}}=$ $31.0 \mathrm{~min}$.

trans-(4R,5S)-3pa (major diastereomer): white solid, m.p. $186-189{ }^{\circ} \mathrm{C}$; $[\alpha]_{\mathrm{D}}{ }^{25}-46.1$ (c 0.90 , $\mathrm{CHCl}_{3}, 84 \%$ ee); ${ }^{1} \mathbf{H}$ NMR $\left(300 \mathrm{MHz}, \mathrm{CDCl}_{3}\right) \delta 7.38(2 \mathrm{H}, \mathrm{d}, J=9.1 \mathrm{~Hz}, \mathrm{Ar}), 7.36-7.27$ (5H, m, Ar), $7.16(2 \mathrm{H}, \mathrm{d}, J=9.1 \mathrm{~Hz}, \mathrm{Ar}), 5.95(1 \mathrm{H}, \mathrm{bs}, \mathrm{NH}), 5.38(1 \mathrm{H}, \mathrm{d}, J=4.3 \mathrm{~Hz}, \mathrm{CH}), 3.98(1 \mathrm{H}, \mathrm{d}, J=$ $4.3 \mathrm{~Hz}, \mathrm{CH}), 1.52$ (9H, s, CH 3$) ;{ }^{13} \mathrm{C}$ NMR (75 MHz, $\left.\mathrm{CDCl}_{3}\right) \delta 169.4$ (C), 158.2 (C), 139.2 (C), $136.9(\mathrm{C}), 129.2(\mathrm{CH}), 128.7(\mathrm{CH}), 128.54(\mathrm{CH}), 128.49(\mathrm{C}), 126.1(\mathrm{CH}), 121.2(\mathrm{CH}), 83.4(\mathrm{C})$, $62.9(\mathrm{CH}), 60.2(\mathrm{CH}), 28.0\left(\mathrm{CH}_{3}\right)$; HRMS (ESI) m/z: $373.1296[\mathrm{M}+\mathrm{H}]^{+}, \mathrm{C}_{20} \mathrm{H}_{22} \mathrm{ClN}_{2} \mathrm{O}_{3}{ }^{+}$requires 373.1313 .

cis-3pa (minor diastereomer): white solid, m.p. 202-206 ${ }^{\circ} \mathrm{C}$; $[\alpha]_{\mathrm{D}}{ }^{25}-8.4\left(\right.$ c $0.66, \mathrm{CHCl}_{3}, 6 \%$ ee); ${ }^{1}$ H NMR (300 MHz, $\left.\mathrm{CDCl}_{3}\right) \delta 7.34(2 \mathrm{H}, \mathrm{d}, J=9.1 \mathrm{~Hz}, \mathrm{Ar}), 7.31-7.27$ (5H, m, Ar), 7.14 (2H, d, $J$ $=9.1 \mathrm{~Hz}, \mathrm{Ar}), 5.46(1 \mathrm{H}, \mathrm{d}, J=9.7 \mathrm{~Hz}, \mathrm{CH}), 5.20(1 \mathrm{H}, \mathrm{bs}, \mathrm{NH}), 4.69(1 \mathrm{H}, \mathrm{d}, J=9.6 \mathrm{~Hz}, \mathrm{CH}), 1.05$ (9H, s, $\left.\mathrm{CH}_{3}\right) ; 167.3(\mathrm{C}), 158.2(\mathrm{C}), 136.8(\mathrm{C}), 135.4(\mathrm{C}), 128.9(\mathrm{C}), 128.7(\mathrm{CH}), 128.7(\mathrm{CH}), 128.0$ 
$(\mathrm{CH}), 121.5(\mathrm{CH}), 82.8(\mathrm{C}), 62.5(\mathrm{CH}), 57.3(\mathrm{CH}), 27.3(\mathrm{CH})$; HRMS (ESI) m/z: 373.1296 $[\mathrm{M}+\mathrm{H}]^{+}, \mathrm{C}_{20} \mathrm{H}_{22} \mathrm{ClN}_{2} \mathrm{O}_{3}{ }^{+}$requires 373.1313 .

tert-Butyl (4R,5S)-1-(4-methoxyphenyl)-2-oxo-5-phenylimidazolidine-4-carboxylate (3qa)

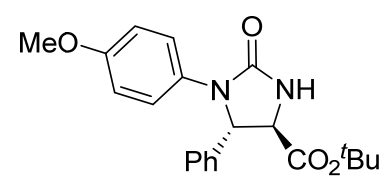

Obtained $22.3 \mathrm{mg}(24 \%)$ from $57.0 \mathrm{mg}(0.25 \mathrm{mmol})$ of 1q. The enantiomeric excess (minor isomer: 2\%, major isomer 91\%) was determined by HPLC (Amylose 1), hexane:iPrOH 80:20, $1 \mathrm{~mL} \cdot \mathrm{min}^{-1}$, cis-3qa (minor diastereomer): minor enantiomer, $\mathrm{t}_{\mathrm{r}}=14.8 \mathrm{~min}$, major enantiomer, $\mathrm{t}_{\mathrm{r}}=31.0 \mathrm{~min}$, trans-(4R,5S)-3qa (major diastereomer): minor enantiomer, $\mathrm{t}_{\mathrm{r}}=33.3 \mathrm{~min}$, major enantiomer, $\mathrm{t}_{\mathrm{r}}=$ $58.0 \mathrm{~min}$.

trans-(4R,5S)-3qa (major diastereomer): white solid, m.p. $177-179{ }^{\circ} \mathrm{C} ;[\alpha]_{\mathrm{D}}{ }^{25}-24.1($ c 0.87 , $\mathrm{CHCl}_{3}, 91 \%$ ee); ${ }^{1} \mathbf{H}$ NMR (300 MHz, $\left.\mathrm{CDCl}_{3}\right) \delta$ 7.39-7.31 (4H, m, Ar), $7.27(2 \mathrm{H}, \mathrm{d}, J=9.1 \mathrm{~Hz}$, Ar), $6.76(2 \mathrm{H}, \mathrm{d}, J=9.1 \mathrm{~Hz}, \mathrm{Ar}), 5.32(1 \mathrm{H}, \mathrm{d}, J=4.5 \mathrm{~Hz}, \mathrm{CH}), 5.27(1 \mathrm{H}, \mathrm{bs}, \mathrm{NH}), 3.98(1 \mathrm{H}, \mathrm{d}, J=$ $4.5 \mathrm{~Hz}, \mathrm{CH}), 3.71\left(3 \mathrm{H}, \mathrm{s}, \mathrm{CH}_{3}\right), 1.52\left(9 \mathrm{H}, \mathrm{s}, \mathrm{CH}_{3}\right) ;{ }^{13} \mathrm{C}$ NMR $\left(75 \mathrm{MHz}, \mathrm{CDCl}_{3}\right) \delta 169.6(\mathrm{C}), 158.6$ (C), $156.2(\mathrm{C}), 139.7(\mathrm{C}), 131.1(\mathrm{C}), 129.1(\mathrm{CH}), 128.4(\mathrm{CH}), 126.4(\mathrm{CH}), 122.9(\mathrm{CH}), 114.1(\mathrm{CH})$, $83.3(\mathrm{C}), 63.9(\mathrm{CH}), 60.3(\mathrm{CH}), 55.3\left(\mathrm{CH}_{3}\right), 28.0\left(\mathrm{CH}_{3}\right)$; HRMS (ESI) m/z: $369.1806[\mathrm{M}+\mathrm{H}]^{+}$, $\mathrm{C}_{21} \mathrm{H}_{25} \mathrm{~N}_{2} \mathrm{O}_{4}{ }^{+}$requires 369.1809 .

cis-3qa(minor diastereomer): white solid, m.p. $171-179{ }^{\circ} \mathrm{C}$; $[\alpha]_{\mathrm{D}}^{25}-2.0\left(\right.$ c $0.37, \mathrm{CHCl}_{3}, 2 \%$ ee); ${ }^{1} \mathbf{H}$ NMR $\left(300 \mathrm{MHz}, \mathrm{CDCl}_{3}\right) \delta$ 7.33-7.25 (5H, m, Ar), $7.23(2 \mathrm{H}, \mathrm{d}, J=9.1 \mathrm{~Hz}, \mathrm{Ar}), 6.74(2 \mathrm{H}, \mathrm{d}, J$ $=9.1 \mathrm{~Hz}, \mathrm{Ar}), 5.42(1 \mathrm{H}, \mathrm{d}, J=9.7 \mathrm{~Hz}, \mathrm{CH}), 5.05(1 \mathrm{H}, \mathrm{bs}, \mathrm{NH}), 4.69(1 \mathrm{H}, \mathrm{d}, J=9.7 \mathrm{~Hz}, \mathrm{CH}), 3.70$ $\left(3 \mathrm{H}, \mathrm{s}, \mathrm{CH}_{3}\right), 1.04\left(9 \mathrm{H}, \mathrm{s}, \mathrm{CH}_{3}\right) ;{ }^{13} \mathrm{C}$ NMR (75 MHz, CDCl 3$) \delta 167.7$ (C), 159.0 (C), 156.2 (C), $136.0(\mathrm{C}), 131.0(\mathrm{C}), 128.6(\mathrm{CH}), 128.5(\mathrm{CH}), 128.1(\mathrm{CH}), 123.3(\mathrm{CH}), 114.0(\mathrm{CH}), 82.4(\mathrm{C}), 63.3$ $(\mathrm{CH}), 57.6(\mathrm{CH}), 55.3\left(\mathrm{CH}_{3}\right), 27.3\left(\mathrm{CH}_{3}\right)$; HRMS (ESI) m/z: $369.1806[\mathrm{M}+\mathrm{H}]^{+}, \mathrm{C}_{21} \mathrm{H}_{25} \mathrm{~N}_{2} \mathrm{O}_{4}{ }^{+}$ requires 369.1809 .

\section{Benzyl (4R,5S)-2-oxo-1,5-diphenylimidazolidine-4-carboxylate (3ab)}

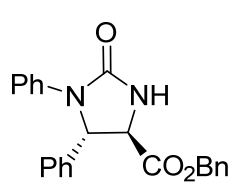

Obtained $60.0 \mathrm{mg}(60 \%)$ from $49.5 \mathrm{mg}(0.25 \mathrm{mmol})$ of 1a and $57.9 \mathrm{mg}(0.33$ mmol) of benzyl isocyanoacetate (2b). The enantiomeric excess (minor isomer:

$23 \%$, major isomer $82 \%$ ) was determined by HPLC (Amylose 1), hexane:iPrOH 80:20, $1 \mathrm{~mL} \cdot \mathrm{min}^{-1}$, cis-3ab (minor diastereomer): minor enantiomer, $\mathrm{t}_{\mathrm{r}}=26.9 \mathrm{~min}$, major enantiomer, $\mathrm{t}_{\mathrm{r}}=14.8 \mathrm{~min}$, trans-(4R,5S)-3ab (major diastereomer): minor enantiomer, $\mathrm{t}_{\mathrm{r}}=29.0$ $\min$, major enantiomer, $\mathrm{t}_{\mathrm{r}}=35.2 \mathrm{~min}$.

trans-(4R,5S)-3ab (major diastereomer): white solid, m.p. $145-152{ }^{\circ} \mathrm{C} ;[\alpha]_{\mathrm{D}}{ }^{25}-69.6(c \quad 0.80$, $\mathrm{CHCl}_{3}, 82 \%$ ee); ${ }^{1} \mathbf{H}$ NMR $\left(300 \mathrm{MHz}, \mathrm{CDCl}_{3}\right) \delta 7.40(2 \mathrm{H}, \mathrm{d}, J=8.8,1.1 \mathrm{~Hz}, \mathrm{Ar}), 7.37$ (5H, s, Ar), 
7.35-7.28 (5H, m, Ar), 7.24-7.18 (2H, m, Ar), 7.00 (1H, t, J = 7.4 Hz, Ar), $5.80(1 \mathrm{H}, \mathrm{bs}, \mathrm{NH}), 5.45$ $(1 \mathrm{H}, \mathrm{d}, J=3.8 \mathrm{~Hz}, \mathrm{CH}), 5.30(1 \mathrm{H}, \mathrm{d}, J=12.1 \mathrm{~Hz}, \mathrm{CH}), 5.23(1 \mathrm{H}, \mathrm{d}, J=12.1 \mathrm{~Hz}, \mathrm{CH}), 4.11(1 \mathrm{H}, \mathrm{d}$, $J=3.8 \mathrm{~Hz}, \mathrm{CH}) ;{ }^{13} \mathrm{C}$ NMR (75 MHz, $\left.\mathrm{CDCl}_{3}\right) \delta 174.5$ (C), 158.3 (C), 139.2 (C), 138.1 (C), 134.8 (C), $129.2(\mathrm{CH}), 128.7(\mathrm{CH}), 128.5(\mathrm{CH}), 128.5(\mathrm{CH}), 128.4(\mathrm{CH}), 126.1(\mathrm{CH}), 123.6(\mathrm{CH})$, $120.1(\mathrm{CH}), 67.8\left(\mathrm{CH}_{2}\right), 63.0(\mathrm{CH}), 59.9(\mathrm{CH})$; HRMS (ESI) m/z: $373.1528[\mathrm{M}+\mathrm{H}]^{+}, \mathrm{C}_{23} \mathrm{H}_{21} \mathrm{~N}_{2} \mathrm{O}_{3}{ }^{+}$ requires 373.1547 .

cis-3ab (minor diastereomer): white solid, m.p. 195-200 ${ }^{\circ} \mathrm{C}$; $[\alpha]_{\mathrm{D}}{ }^{25}+1.9\left(\mathrm{c} 0.61, \mathrm{CHCl}_{3}, 23 \%\right.$ ee); ${ }^{1} \mathbf{H}$ NMR $\left(300 \mathrm{MHz}, \mathrm{CDCl}_{3}\right) \delta 7.38(2 \mathrm{H}, \mathrm{dd}, J=8.7,1.0 \mathrm{~Hz}, \mathrm{Ar}), 7.35-7.26(8 \mathrm{H}, \mathrm{m}, \mathrm{Ar}), 7.25-7.19$ $(2 \mathrm{H}, \mathrm{m}, \mathrm{Ar}), 7.09$ (2H, dd, $J=6.6,3.0 \mathrm{~Hz}, \mathrm{Ar}), 7.00(1 \mathrm{H}, \mathrm{t}, J=7.4 \mathrm{~Hz}, \mathrm{Ar}), 5.59(1 \mathrm{H}, \mathrm{d}, J=9.5$ $\mathrm{Hz}, \mathrm{CH}), 5.54(1 \mathrm{H}, \mathrm{bs}, \mathrm{NH}), 4.83(1 \mathrm{H}, \mathrm{d}, J=12.0 \mathrm{~Hz}, \mathrm{CH}), 4.81(1 \mathrm{H}, \mathrm{d}, J=9.5 \mathrm{~Hz}, \mathrm{CH}), 4.41(1 \mathrm{H}$, d, $J=12.0 \mathrm{~Hz}, \mathrm{CH}) ;{ }^{13} \mathrm{C}$ NMR $\left(75 \mathrm{MHz}, \mathrm{CDCl}_{3}\right) \delta 168.8$ (C), 158.8 (C), 137.9 (C), 135.1 (C), $134.4(\mathrm{C}), 128.8(\mathrm{CH}), 128.6(\mathrm{CH}), 128.54(\mathrm{CH}), 128.49(\mathrm{CH}), 127.5(\mathrm{CH}), 123.8(\mathrm{CH}), 120.7$ $(\mathrm{CH}), 67.4\left(\mathrm{CH}_{2}\right), 62.6(\mathrm{CH}), 57.6(\mathrm{CH})$; HRMS (ESI) m/z: $[\mathrm{M}+\mathrm{H}]^{+}, \mathrm{C}_{23} \mathrm{H}_{21} \mathrm{~N}_{2} \mathrm{O}_{3}{ }^{+}$requires 373.1547 .

\section{Methyl (4R,5S)-2-oxo-1,5-diphenylimidazolidine-4-carboxylate (3ac)}

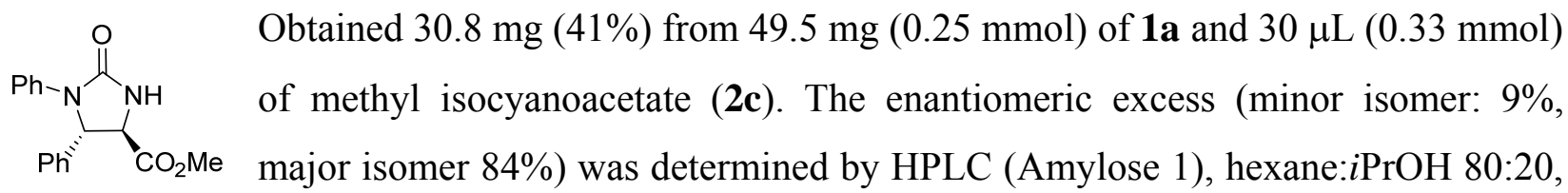

$1 \mathrm{~mL} \cdot \mathrm{min}^{-1}$, cis-3ac (minor diastereomer): minor enantiomer, $\mathrm{t}_{\mathrm{r}}=19.3 \mathrm{~min}$, major enantiomer, $\mathrm{t}_{\mathrm{r}}$ $=12.1 \mathrm{~min}$, trans-(4R,5S)-3ac (major diastereomer): minor enantiomer, $\mathrm{t}_{\mathrm{r}}=33.2 \mathrm{~min}$, major enantiomer, $\mathrm{t}_{\mathrm{r}}=23.6 \mathrm{~min}$.

trans-(4R,5S)-3ac (major diastereomer): white solid, m.p. $84-91^{\circ} \mathrm{C} ;[\alpha]_{\mathrm{D}}^{25}-39.3\left(\right.$ c $1.0, \mathrm{CHCl}_{3}$, 84\% ee); ${ }^{1} \mathbf{H}$ NMR (300 MHz, $\left.\mathrm{CDCl}_{3}\right) \delta 7.43(2 \mathrm{H}, \mathrm{dd}, J=8.8,1.1 \mathrm{~Hz}, \mathrm{Ar}), 7.40-7.27$ (5H, m, Ar), 7.25-7.19 (2H, m, Ar), 6.99 (1H, t, $J=6.8 \mathrm{~Hz}, \mathrm{Ar}), 5.63(1 \mathrm{H}, \mathrm{bs}, \mathrm{NH}), 5.47(1 \mathrm{H}, \mathrm{d}, J=3.5 \mathrm{~Hz}$, $\mathrm{CH}), 4.07(1 \mathrm{H}, \mathrm{d}, J=3.5 \mathrm{~Hz}, \mathrm{CH}), 3.85\left(3 \mathrm{H}, \mathrm{s}, \mathrm{CH}_{3}\right) ;{ }^{13} \mathbf{C ~ N M R}\left(75 \mathrm{MHz}, \mathrm{CDCl}_{3}\right) \delta 171.2(\mathrm{C})$, $158.3(\mathrm{C}), 139.3(\mathrm{C}), 138.1(\mathrm{C}), 129.2(\mathrm{CH}), 128.7(\mathrm{CH}), 128.5(\mathrm{CH}), 126.0(\mathrm{CH}), 123.6(\mathrm{CH})$, $120.0(\mathrm{CH}), 63.0(\mathrm{CH}), 59.7(\mathrm{CH}), 53.1\left(\mathrm{CH}_{3}\right)$; HRMS (ESI) m/z: $297.1222[\mathrm{M}+\mathrm{H}]^{+}, \mathrm{C}_{17} \mathrm{H}_{17} \mathrm{~N}_{2} \mathrm{O}_{3}{ }^{+}$ requires 297.1234 .

cis-3ac (minor diastereomer): white solid, m.p. $178-180{ }^{\circ} \mathrm{C}$; $[\alpha]_{\mathrm{D}}{ }^{25}+5.0\left(\right.$ c $0.34, \mathrm{CHCl}_{3}, 9 \%$ ee); ${ }^{1} \mathbf{H}$ NMR $\left(300 \mathrm{MHz}, \mathrm{CDCl}_{3}\right) \delta 7.37(2 \mathrm{H}, \mathrm{dd}, J=8.7,1.1 \mathrm{~Hz}, \mathrm{Ar}), 7.30-7.26$ (5H, m, Ar), 7.24-7.17 $(2 \mathrm{H}, \mathrm{m}, \mathrm{Ar}), 6.99(1 \mathrm{H}, \mathrm{t}, J=7.4 \mathrm{~Hz}, \mathrm{Ar}), 5.58(1 \mathrm{H}, \mathrm{d}, J=9.5 \mathrm{~Hz}, \mathrm{CH}), 5.31(1 \mathrm{H}, \mathrm{bs}, \mathrm{NH}), 4.77$ $(1 \mathrm{H}, \mathrm{d}, J=4.77 \mathrm{~Hz}, \mathrm{CH}), 3.25\left(3 \mathrm{H}, \mathrm{s}, \mathrm{CH}_{3}\right) ;{ }^{13} \mathrm{C} \mathrm{NMR}\left(75 \mathrm{MHz}, \mathrm{CDCl}_{3}\right) \delta 169.3(\mathrm{C}), 158.8(\mathrm{C})$, 
$137.9(\mathrm{C}), 135.2(\mathrm{C}), 128.8(\mathrm{CH}), 128.7(\mathrm{CH}), 128.6(\mathrm{CH}), 123.8(\mathrm{CH}), 120.6(\mathrm{CH}), 62.6(\mathrm{CH})$, $57.6(\mathrm{CH}), 52.0\left(\mathrm{CH}_{3}\right)$; HRMS (ESI) m/z: $[\mathrm{M}+\mathrm{H}]^{+}, \mathrm{C}_{17} \mathrm{H}_{17} \mathrm{~N}_{2} \mathrm{O}_{3}{ }^{+}$requires 297.1234.

\section{(4R,5S)-2-Oxo-1,5-diphenylimidazolidine-4-carboxylic acid (4)}

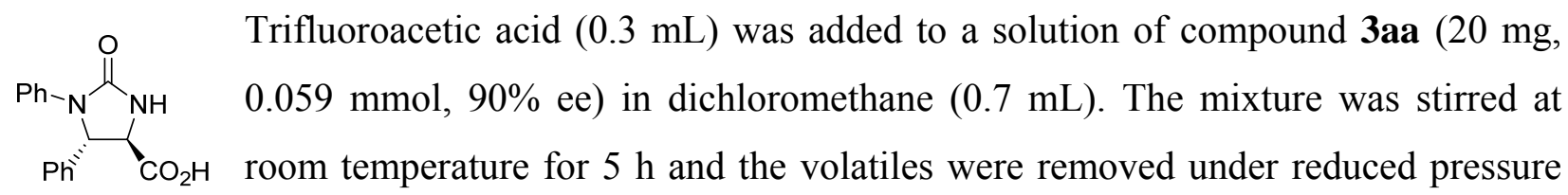
to give $14.1 \mathrm{mg}(89 \%)$ of acid 4. White solid, m.p. $166^{\circ} \mathrm{C}$ (dec.); $[\alpha]_{\mathrm{D}}{ }^{25}-44.5$ (c 1.0, $\mathrm{MeOH}, 90 \%$ ee); ${ }^{1} \mathbf{H}$ NMR (300 MHz, MeOD) $\delta 10.16(1 \mathrm{H}, \mathrm{s}, \mathrm{COOH}), 7.38-7.26(7 \mathrm{H}, \mathrm{m}, \mathrm{Ar}), 7.19$ (2H, t, $J=$ $7.0 \mathrm{~Hz}, \mathrm{Ar}), 6.99(1 \mathrm{H}, \mathrm{t}, J=7.2 \mathrm{~Hz}, \mathrm{Ar}), 5.53(1 \mathrm{H}, \mathrm{bs}, \mathrm{CH}), 4.14(1 \mathrm{H}, \mathrm{bs}, \mathrm{CH}) ;{ }^{13} \mathrm{C}$ NMR (125 $\left.\mathrm{MHz}, \mathrm{CDCl}_{3}\right) 173.7(\mathrm{C}), 160.8(\mathrm{C}), 139.1(\mathrm{C}), 137.3(\mathrm{C}), 129.2(\mathrm{CH}), 128.8(\mathrm{CH}), 128.6(\mathrm{CH})$, $126.2(\mathrm{CH}), 124.4(\mathrm{CH}), 121.1(\mathrm{CH}), 63.5(\mathrm{CH}), 60.4(\mathrm{CH})$; HRMS (ESI) m/z: $283.1076[\mathrm{M}+\mathrm{H}]^{+}$, $\mathrm{C}_{16} \mathrm{H}_{15} \mathrm{~N}_{2} \mathrm{O}_{3}{ }^{+}$requires 283.1077 .

\section{(4R,5S)-4-(Hydroxymethyl)-1,5-diphenylimidazolidin-2-one (5)}

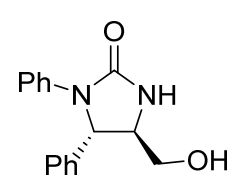

A $2 \mathrm{M}$ solution of $\mathrm{BH}_{3} \cdot \mathrm{SMe}_{2}$ in THF $(75 \mu \mathrm{L}, 0.149 \mathrm{mmol})$ was added to a solution of acid 4 in dry THF (1 mL) under $\mathrm{N}_{2}$ atmosphere. The mixture was stirred at room temperature for $24 \mathrm{~h}$. The reaction was quenched with $\mathrm{MeOH}(3$ $\mathrm{mL}$ ) and the volatiles were removed under reduced pressure, this operation was repeated three additional times. Column chromatography eluting with EtOAc gave $12.1 \mathrm{mg}$ (76\%) of compound 5. The enantiomeric excess was determined by HPLC (Amylose 1) hexane:iPrOH,80:20, $1 \mathrm{~mL} \cdot \min ^{-1}$, minor enantiomer, $\operatorname{tr}=20,4 \mathrm{~min}$, major enantiomer, $\operatorname{tr}=16,5 \mathrm{~min}$. White solid; m.p. $=$ $178-181{ }^{\circ} \mathrm{C} ;[\alpha]_{\mathrm{D}}{ }^{25}-16.9$ (c 0.66, MeOH, 90\% ee); ${ }^{1} \mathbf{H}$ RMN (300 MHz, DMSO) $\delta 7.45$ (2H, d, $J$ $=8.0 \mathrm{~Hz}, \mathrm{Ar}), 7.37-7.12(7 \mathrm{H}, \mathrm{m}, \mathrm{Ar}), 6.89(1 \mathrm{H}, \mathrm{t}, J=7.3 \mathrm{~Hz}, \mathrm{Ar}), 5.19(1 \mathrm{H}, \mathrm{d}, J=3.8 \mathrm{~Hz}, \mathrm{CH})$, $3.99(2 \mathrm{H}, \mathrm{bs}), 3.50(2 \mathrm{H}, \mathrm{d}, J=5.2 \mathrm{~Hz}, \mathrm{CH}), 3.36-3.23(1 \mathrm{H}, \mathrm{m}, \mathrm{CH}) ;{ }^{13} \mathrm{C}$ NMR $(75 \mathrm{MHz}, \mathrm{DMSO})$ $\delta 158.2(\mathrm{C}), 141.4(\mathrm{C}), 139.6(\mathrm{C}), 128.9(\mathrm{CH}), 128.4(\mathrm{CH}), 127.6(\mathrm{CH}), 126.0(\mathrm{CH}), 121.9(\mathrm{CH})$, $118.9(\mathrm{CH}), 63.1\left(\mathrm{CH}_{2}\right), 61.0(\mathrm{CH}), 59.8(\mathrm{CH})$; HRMS (ESI) m/z: 269.1287 [M+H] $]^{+}, \mathrm{C}_{16} \mathrm{H}_{17} \mathrm{~N}_{2} \mathrm{O}_{2}{ }^{+}$ requires 269.1285 .

\section{tert-butyl (4R,5S)-2-oxo-5-phenylimidazolidine-4-carboxylate (6)}

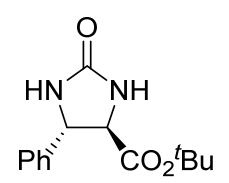

A solution of CAN (101.3 $\mathrm{mg}, 0.185 \mathrm{mmol})$ in water $(0.5 \mathrm{~mL})$ was added to a solution of compound trans-3qa $(22.7 \mathrm{mg}, 0.062 \mathrm{mmol}, 91 \%$ ee) in $\mathrm{MeCN}$ (1.5

$\mathrm{mL}$ ) at $0{ }^{\circ} \mathrm{C}$. The reaction was warmed to room temperature and stirred until consumption of starting material (TLC). Then, the mixture was diluted with EtOAc $(10 \mathrm{~mL})$ and washed with aqueous saturated $\mathrm{NaHCO}_{3}$ and brine, and dried over $\mathrm{Na}_{2} \mathrm{SO}_{4}$. After removing the 
solvent under reduced pressure, column chromatography eluting with hexane:EtOAc (4:6 to 3:7) yielded $12.2 \mathrm{mg}(75 \%)$ of compound 6 . The enantiomeric excess $(88 \%)$ was determined by HPLC (Amylose 1), hexane:iPrOH 80:20, $1 \mathrm{~mL} \cdot \mathrm{min}^{-1} / \mathrm{min}$, minor enantiomer, $\mathrm{t}_{\mathrm{r}}=8.0 \mathrm{~min}$, major enantiomer, $\mathrm{t}_{\mathrm{r}}=12.8 \mathrm{~min}$. White solid; m.p. $175-178{ }^{\circ} \mathrm{C} ;[\alpha]_{\mathrm{D}}{ }^{25}-39.6\left(c 0.81, \mathrm{CHCl}_{3}, 88 \%\right.$ ee $) ;{ }^{1} \mathbf{H}$ NMR (300 MHz, $\left.\mathrm{CDCl}_{3}\right) \delta$ 7.44-7.29 (5H, m, Ar), $5.54(2 \mathrm{H}, \mathrm{bs}, 2 \times \mathrm{NH}), 4.93(1 \mathrm{H}, \mathrm{d}, J=5.1 \mathrm{~Hz}$, $\mathrm{CH}), 3.99(1 \mathrm{H}, \mathrm{d}, J=5.1 \mathrm{~Hz}, \mathrm{CH}), 1.50\left(9 \mathrm{H}, \mathrm{s}, \mathrm{CH}_{3}\right) ;{ }^{13} \mathbf{C ~ N M R}\left(75 \mathrm{MHz}, \mathrm{CDCl}_{3}\right) \delta 169.7(\mathrm{C})$, $162.0(\mathrm{C}), 141.2(\mathrm{C}), 128.9(\mathrm{CH}), 128.4(\mathrm{CH}), 126.1(\mathrm{CH}), 83.0(\mathrm{C}), 62.9(\mathrm{CH}), 59.1(\mathrm{CH}), 28.0$ $\left(\mathrm{CH}_{3}\right)$; HRMS (ESI) m/z: $263.1378[\mathrm{M}+\mathrm{H}]^{+}, \mathrm{C}_{14} \mathrm{H}_{19} \mathrm{~N}_{2} \mathrm{O}_{3}{ }^{+}$requires 263.1390.

\section{(4R,5S)-2-Oxo-5-phenylimidazolidine-4-carboxylic acid (7)}

O Trifluoroacetic acid $(0.3 \mathrm{~mL})$ was added to a solution of compound 4 (11 $\mathrm{mg}$, $0.042 \mathrm{mmol}, 89 \%$ ee) in $\mathrm{DCM}(0.6 \mathrm{~mL})$. The mixture was stirred at room

$\mathrm{Ph} \quad \mathrm{CO}_{2} \mathrm{H}$ temperature for $7 \mathrm{~h}$ and the volatiles were removed under reduced pressure to give $7.5 \mathrm{mg}(87 \%)$ of acid 5. ${ }^{1} \mathbf{H}$ NMR (300 MHz, MeOD) $\delta$ 7.42-7.29 (5H, m, Ar), $4.88(1 \mathrm{H}, \mathrm{d}, J=4.6$ $\mathrm{Hz}, \mathrm{CH}), 4.11(1 \mathrm{H}, \mathrm{d}, J=4.6 \mathrm{~Hz}, \mathrm{CH}) ;{ }^{13} \mathrm{C}$ NMR $(75 \mathrm{MHz}, \mathrm{MeOD}) \delta 173.3(\mathrm{C}), 165.0$ (C), 143.1 (C), $129.9 \mathrm{CH}), 129.4(\mathrm{CH}), 126.7(\mathrm{CH}), 63.8(\mathrm{CH}), 60.5(\mathrm{CH})$.

\section{Methyl (4R,5S)-2-oxo-5-phenylimidazolidine-4-carboxylate (8)}

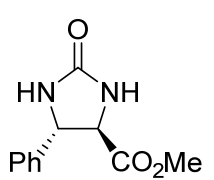

A solution of the crude product $5(7.5 \mathrm{mg}, 0.036 \mathrm{mmol})$ and a drop of concentrated sulfuric acid in $\mathrm{MeOH}(2 \mathrm{~mL})$ was heated at reflux temperature for 6 hours. The mixture was concentrated under reduced pressure, diluted in EtOAc $(30 \mathrm{~mL})$, washed with aqueous saturated $\mathrm{NaHCO}_{3}(5 \mathrm{~mL})$, brine $(5 \mathrm{~mL})$ and dried over $\mathrm{MgSO}_{4}$. After filtering and removing the solvent under reduced pressure $7.1 \mathrm{mg}(89 \%)$ of methyl ester 8 were obtained. The enantiomeric excess (89\%) was determined by HPLC (Amylose 1), hexane:iPrOH $80: 20,1 \mathrm{~mL} \cdot \mathrm{min}^{-1}$, minor enantiomer, $\mathrm{t}_{\mathrm{r}}=8.7 \mathrm{~min}$, major enantiomer, $\mathrm{t}_{\mathrm{r}}=15.4 \mathrm{~min}$. White solid; m.p. $199-206{ }^{\circ} \mathrm{C}$ (dec.); $[\alpha]_{\mathrm{D}}^{25}-85.5$ (c 0.46, MeOH, 89\% ee), Lit. ${ }^{6}[\alpha]_{\mathrm{D}}{ }^{25}-101.7$ (c 1.0, MeOH); ${ }^{1}$ H NMR (300 MHz, MeOD) $\delta$ 7.42-7.29 (5H, m, Ar), 4.88 (1H, d, J=4.6 Hz, CH), 4.11 (1H, d, $J$ $=4.6 \mathrm{~Hz}, \mathrm{CH}) ;{ }^{13} \mathrm{C}$ NMR (75 MHz, MeOD) $\delta 173.3(\mathrm{C}), 165.0(\mathrm{C}), 143.1(\mathrm{C}), 129.9(\mathrm{CH}), 129.4$ $(\mathrm{CH}), 127.0(\mathrm{CH}), 63.8(\mathrm{CH}), 60.5(\mathrm{CH}), 53.1\left(\mathrm{CH}_{3}\right)$; HRMS (ESI) m/z: $263.1378[\mathrm{M}+\mathrm{H}]^{+}$, $\mathrm{C}_{14} \mathrm{H}_{19} \mathrm{~N}_{2} \mathrm{O}_{3}{ }^{+}$requires 263.1390 .

\section{Synthesis of compound 3aa at $1 \mathrm{mmol}$ scale}

Nitrone 1a (197.2 mg, $1 \mathrm{mmol})$ was added to a solution of squaramide SQ4 (58,0 mg, $0.1 \mathrm{mmol})$ and silver oxide $(12.0 \mathrm{mg}, 0.5 \mathrm{mmol})$ in MTBE $(24 \mathrm{~mL})$. tert-Butyl isocyanoacetate 2a $(189 \mu \mathrm{L}$, $1.3 \mathrm{mmol}$ ) was added, the flask was closed with a stopper and the mixture was stirred at room 
temperature for 4 days. After this time, the reaction mixture was filtered through a pad of silica gel $(2 \mathrm{~cm} \times 7 \mathrm{~cm})$ and eluted with $150 \mathrm{~mL}$ of 3:7 hexane:EtOAc to remove the silver salt and concentrated under reduced pressure. The mixture was chromatographed eluting with toluene:ether (9:1 to $8: 2)$ to give $202.4 \mathrm{mg}(60 \%)$ of trans-(4R,5S)-3aa (88\% ee) and $65,7(19 \%)$ of cis-3aa (2\% ee).

\section{References}

1. Jin, C.; Zhang,M.; Wu, L.; Guan, Y.; Pan, Y.; Jiang, J.; Lin, C.; Wang, L. Chem. Commun. 2013, 49, 2025-2027.

2. Yang, W.; Du, D.-M. Org. Biomol. Chem. 2012, 10, 6876-6884.

3. Cassani, C; Martin-Rapun, R.; Arceo, E.; Bravo, F.; Melchiorre, P. Nat. Protoc. 2013, 8, 325344.

4. Tian, Z.; Xu, J.; Liu, B.; Tan, Q.; Xu, B. Org. Lett. 2018, 20, 2603-2606.

5. The synthesis of racemic compounds $\mathbf{3}$ was attempted by using different achiral squaramides without success. Finally we could obtain near racemic compounds by using an equimolar mixture of squaramides derived from quinine and quinidine as organocatalyst.

6. S.-H. Lee, J. Yoon, S.-H. Chunga, Y.-S. Lee. Tetrahedron 2001, 57, 2139-2145. 


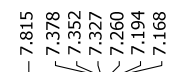

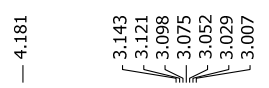

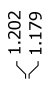

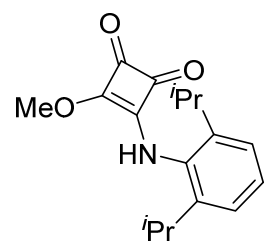

${ }^{1} \mathrm{H} \mathrm{NMR,} \mathrm{CDCl}_{3}, 300 \mathrm{MHz}$

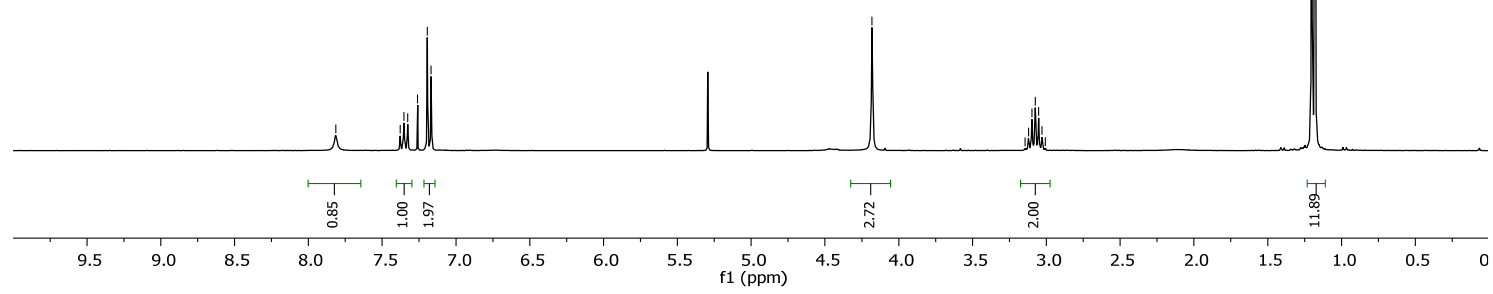

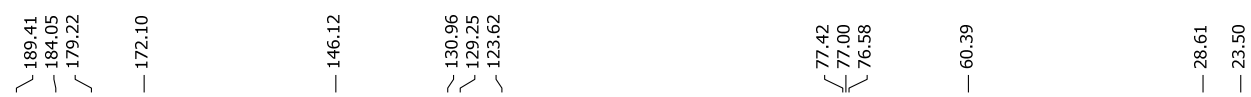

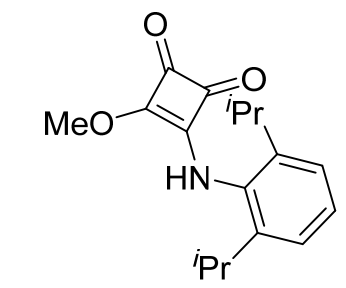

${ }^{13} \mathrm{C} \mathrm{NMR}, \mathrm{CDCl}_{3}, 75 \mathrm{MHz}$

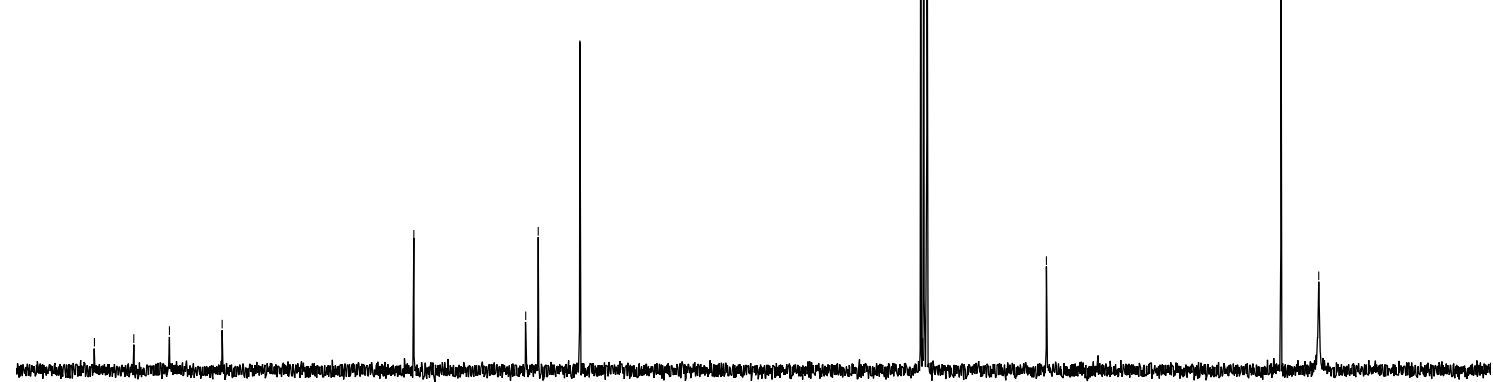

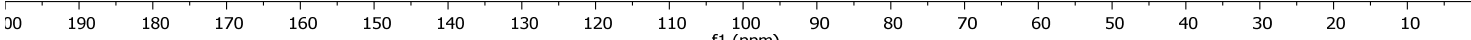



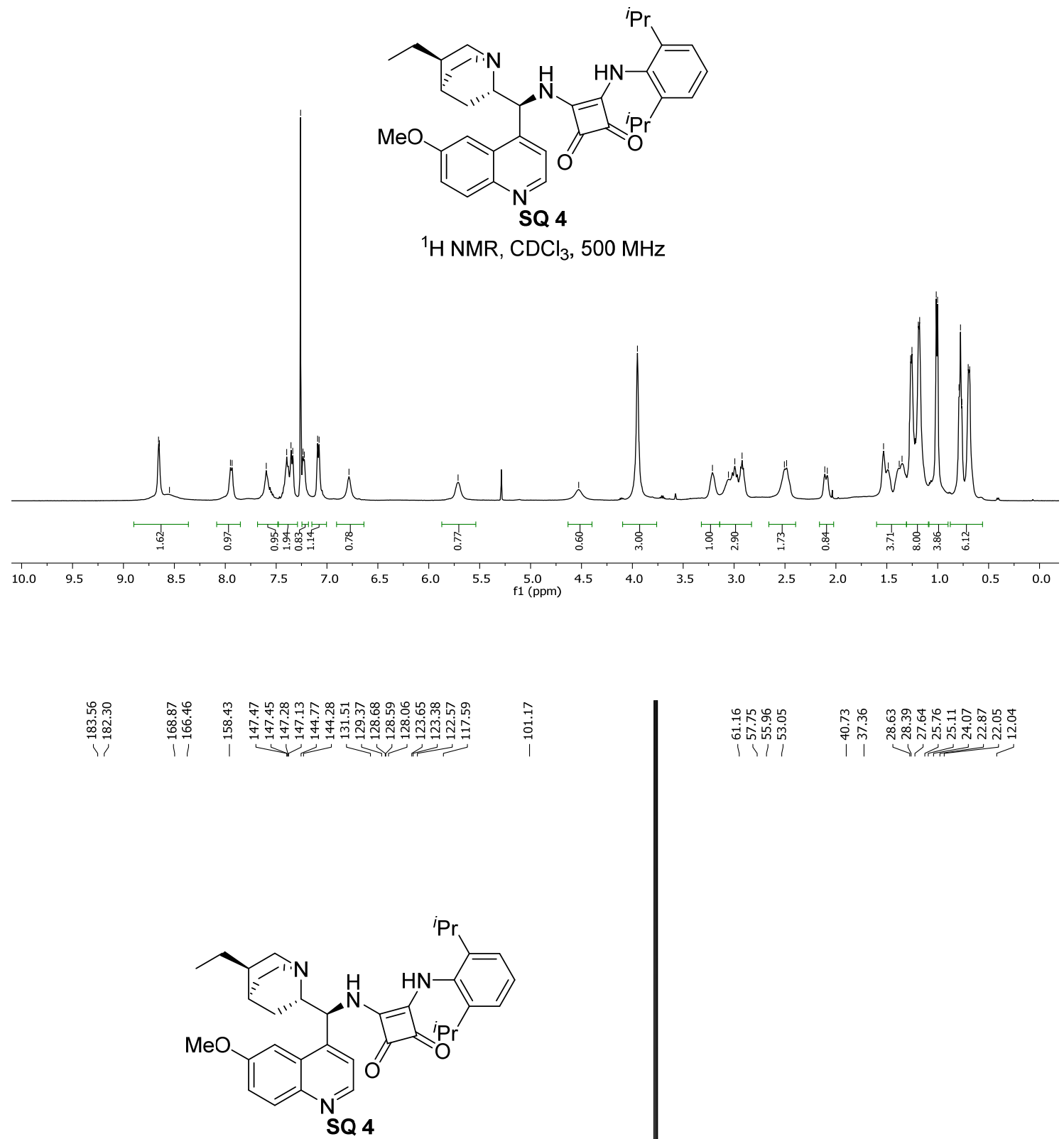

${ }^{13} \mathrm{C} \mathrm{NMR}, \mathrm{CDCl}_{3}, 125 \mathrm{MHz}$
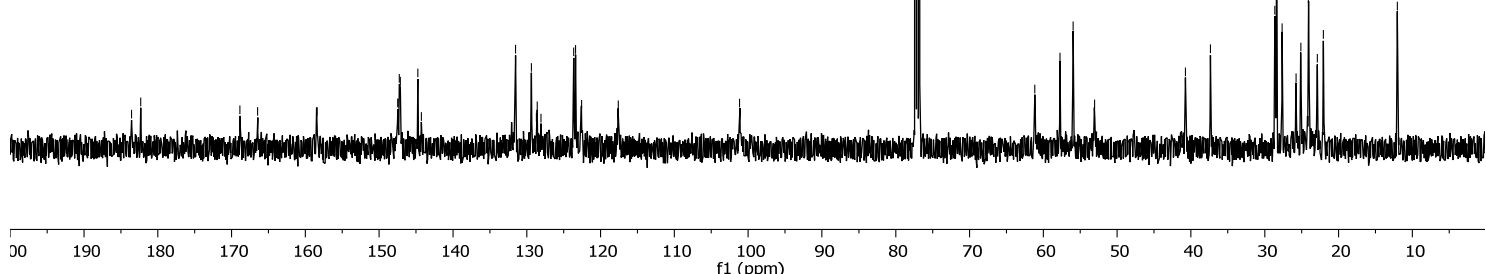
<smiles>CCCCC(=O)C1NC(=O)N(c2ccccc2)C1c1ccccc1</smiles>

trans-(4R,5S)-3aa

${ }^{1} \mathrm{H} \mathrm{NMR}, \mathrm{CDCl}_{3}, 300 \mathrm{MHz}$
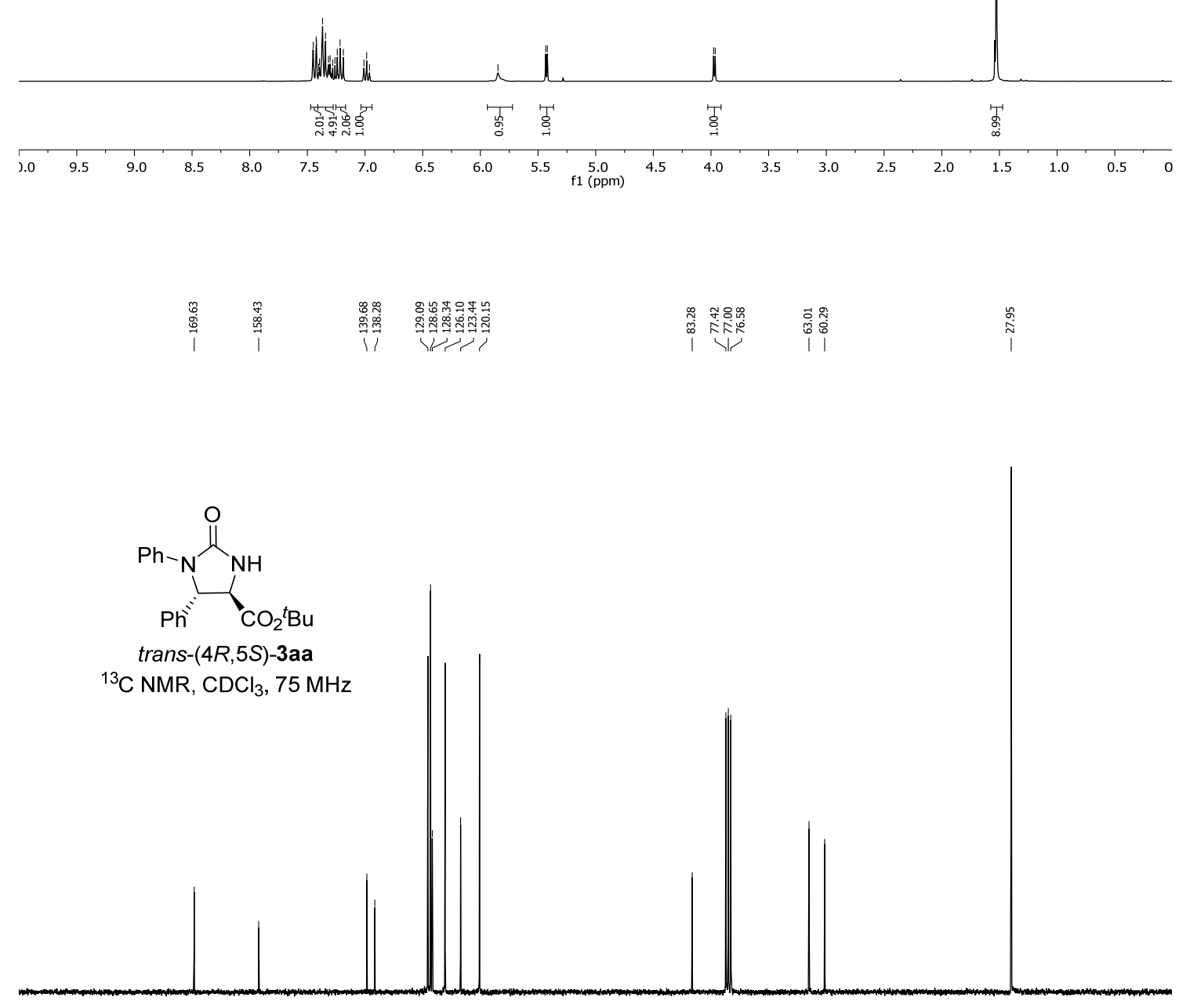

$\begin{array}{llllllllll}190 & 180 & 170 & 160 & 150 & 140 & 130 & 120 & 110 & \begin{array}{l}100 \\ \mathrm{f} 1(\mathrm{ppm})\end{array}\end{array}$

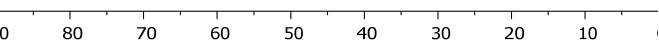




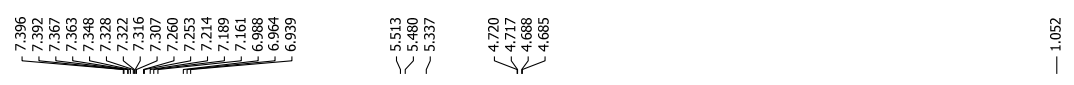

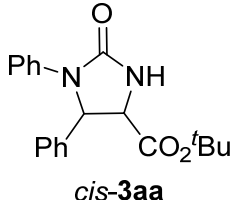

${ }^{1} \mathrm{H} \mathrm{NMR}, \mathrm{CDCl}_{3}, 300 \mathrm{MHz}$

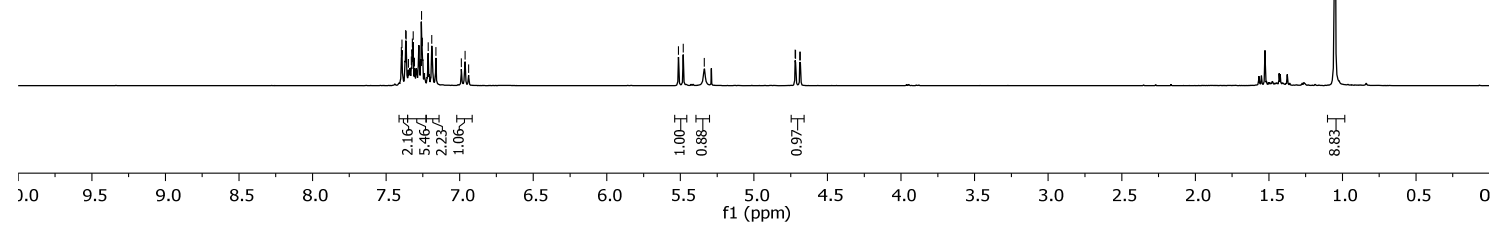

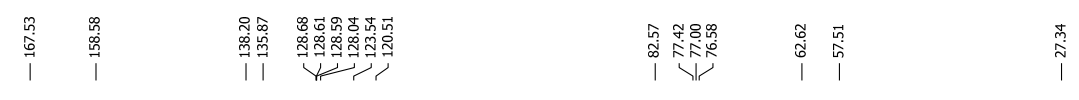

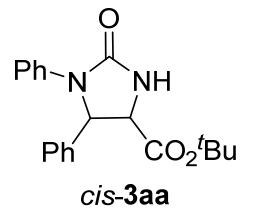

${ }^{13} \mathrm{C} \mathrm{NMR}, \mathrm{CDCl}_{3}, 75 \mathrm{MHz}$

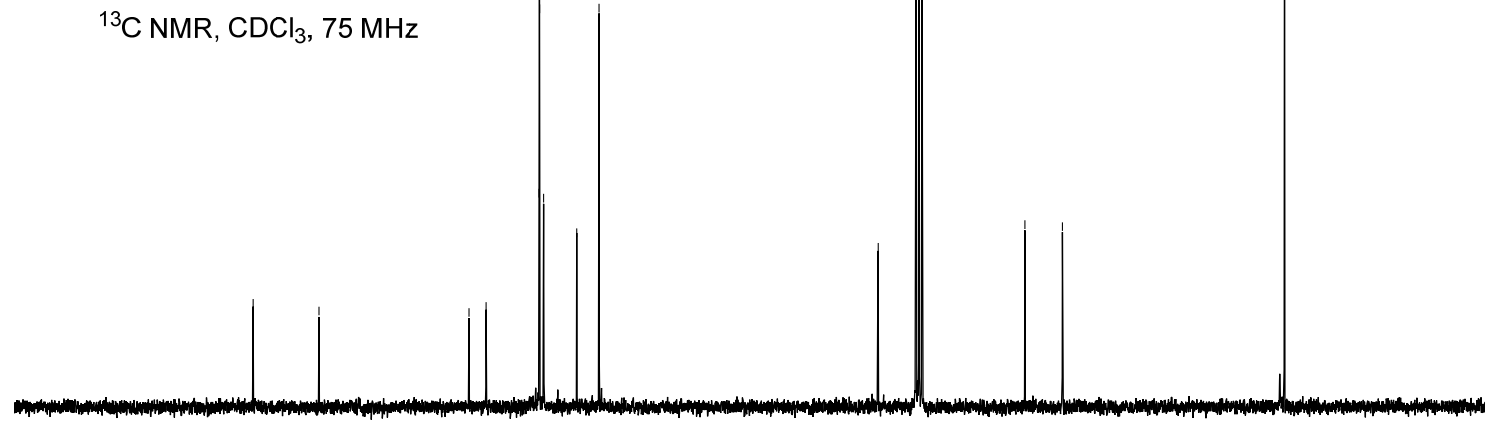

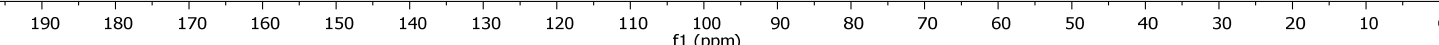




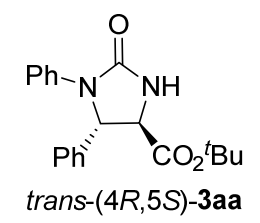

\section{Racemic}

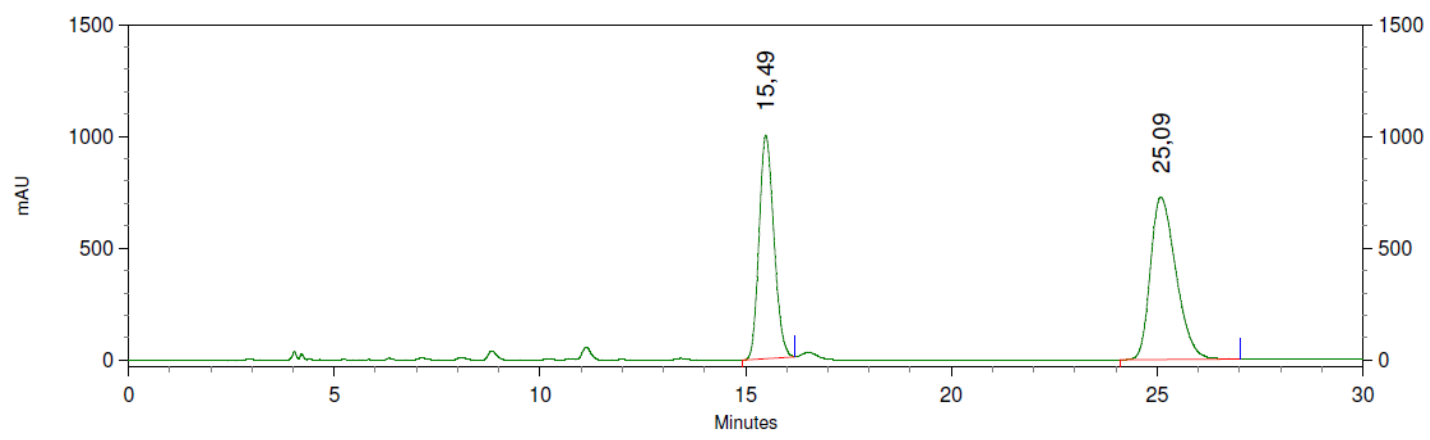

6: 242 nm, 4 nm Results Retention Time

$\begin{array}{lll}15,49 & 100092529 & 44,431 \\ 25,09 & 125185615 & 55,569\end{array}$

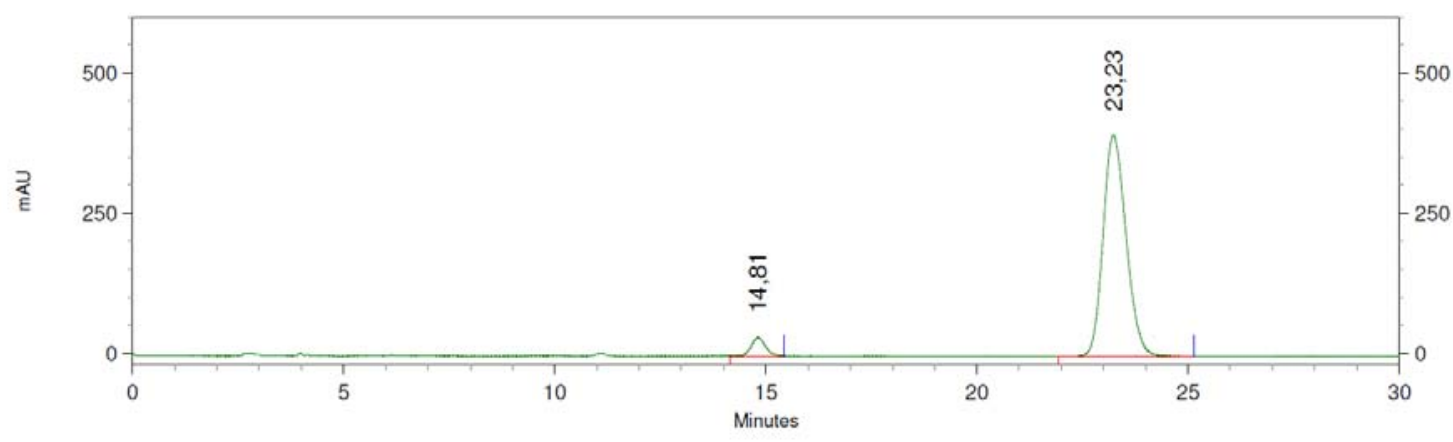

2: $242 \mathrm{~nm}, 4 \mathrm{~nm}$ Results Retention Time Area Area Percent 14,81 3080274 4,915
95,085 

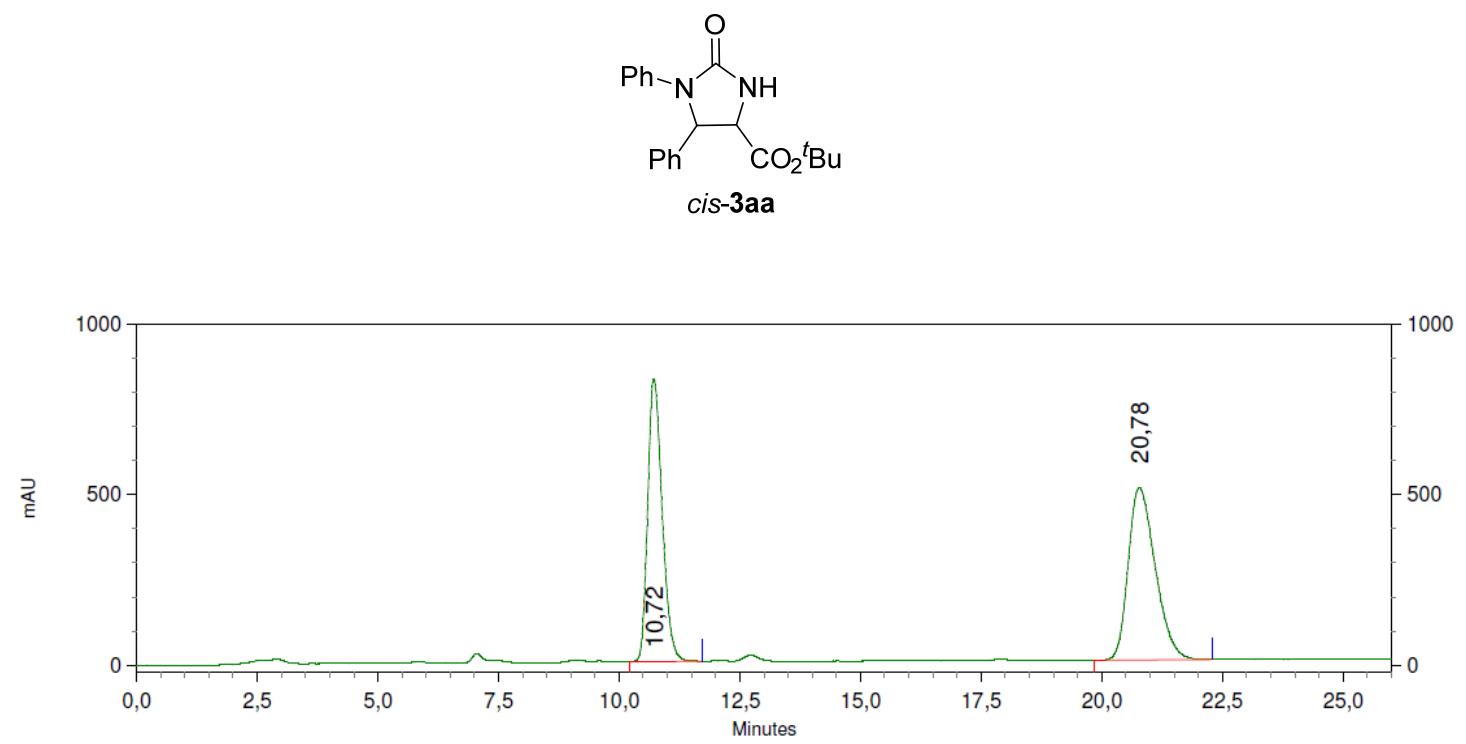

2: $242 \mathrm{~nm}, 4 \mathrm{~nm}$ Results

Retention Time

Area

Area Percent

10,72
20,78 69031861

46,755

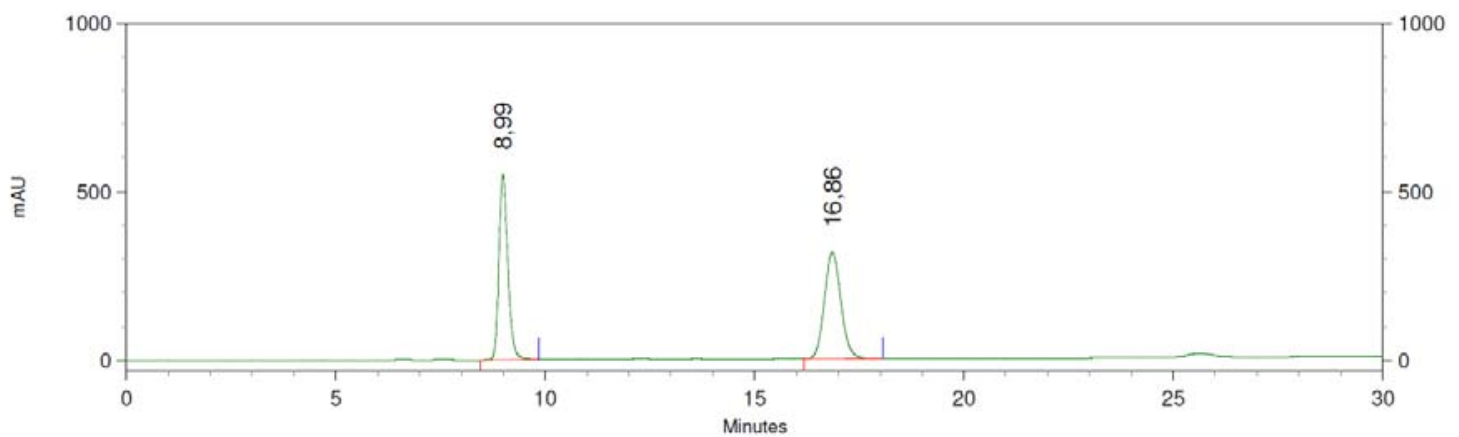

2: $242 \mathrm{~nm}, 4 \mathrm{~nm}$ Results Retention Time Area Area Percent

$\begin{array}{rrr}8,99 & 32522518 & 48,500 \\ 16,86 & 34534847 & 51,500\end{array}$




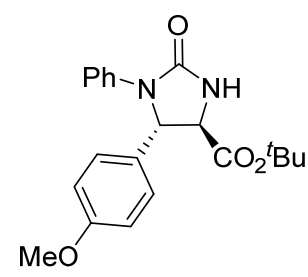

trans-(4R,5S)-3ba

${ }^{1} \mathrm{H} \mathrm{NMR}, \mathrm{CDCl}_{3}, 300 \mathrm{MHz}$

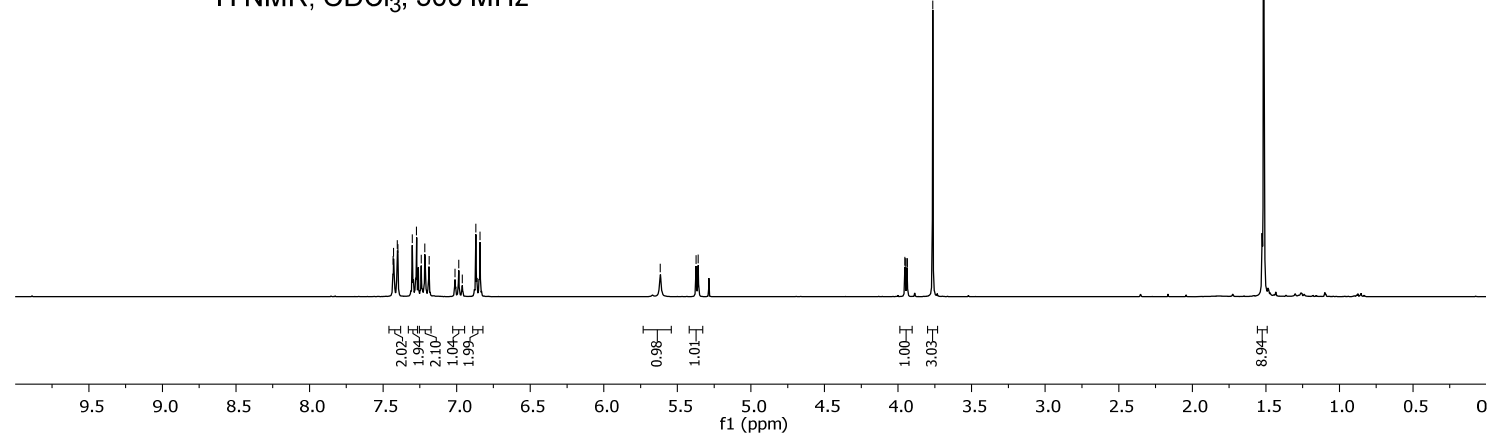

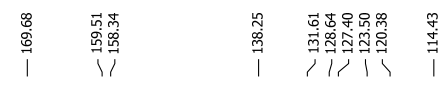

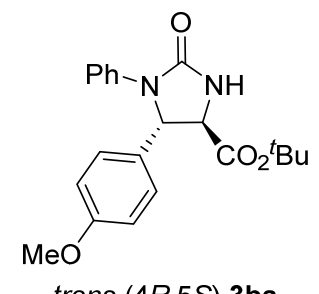

trans-(4R,5S)-3ba

${ }^{13} \mathrm{C} \mathrm{NMR}, \mathrm{CDCl}_{3}, 75 \mathrm{MHz}$

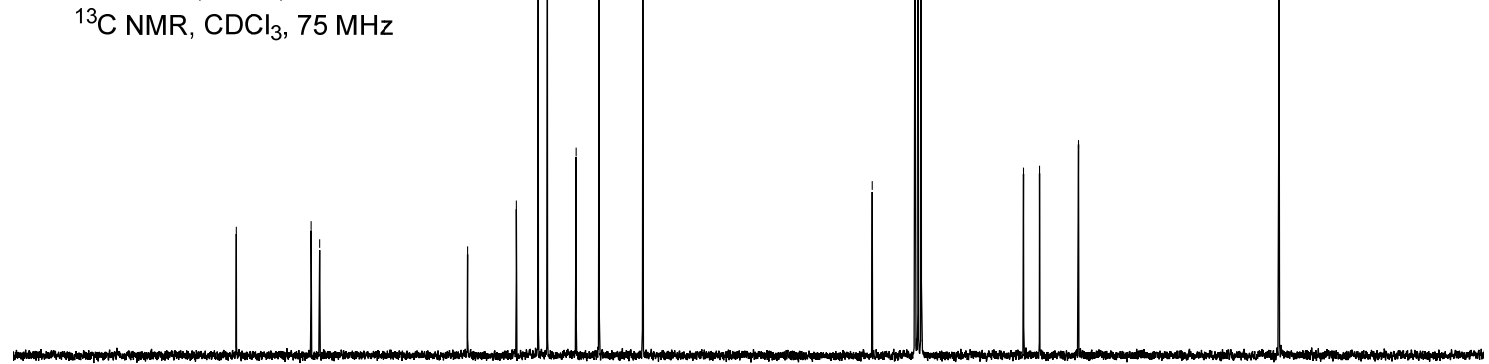

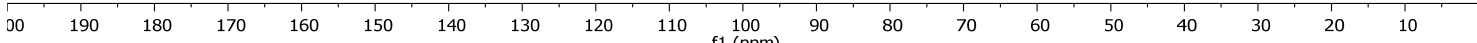




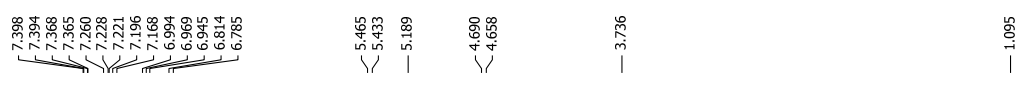

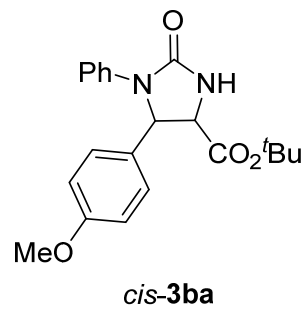

${ }^{1} \mathrm{H} \mathrm{NMR}, \mathrm{CDCl}_{3}, 300 \mathrm{MHz}$
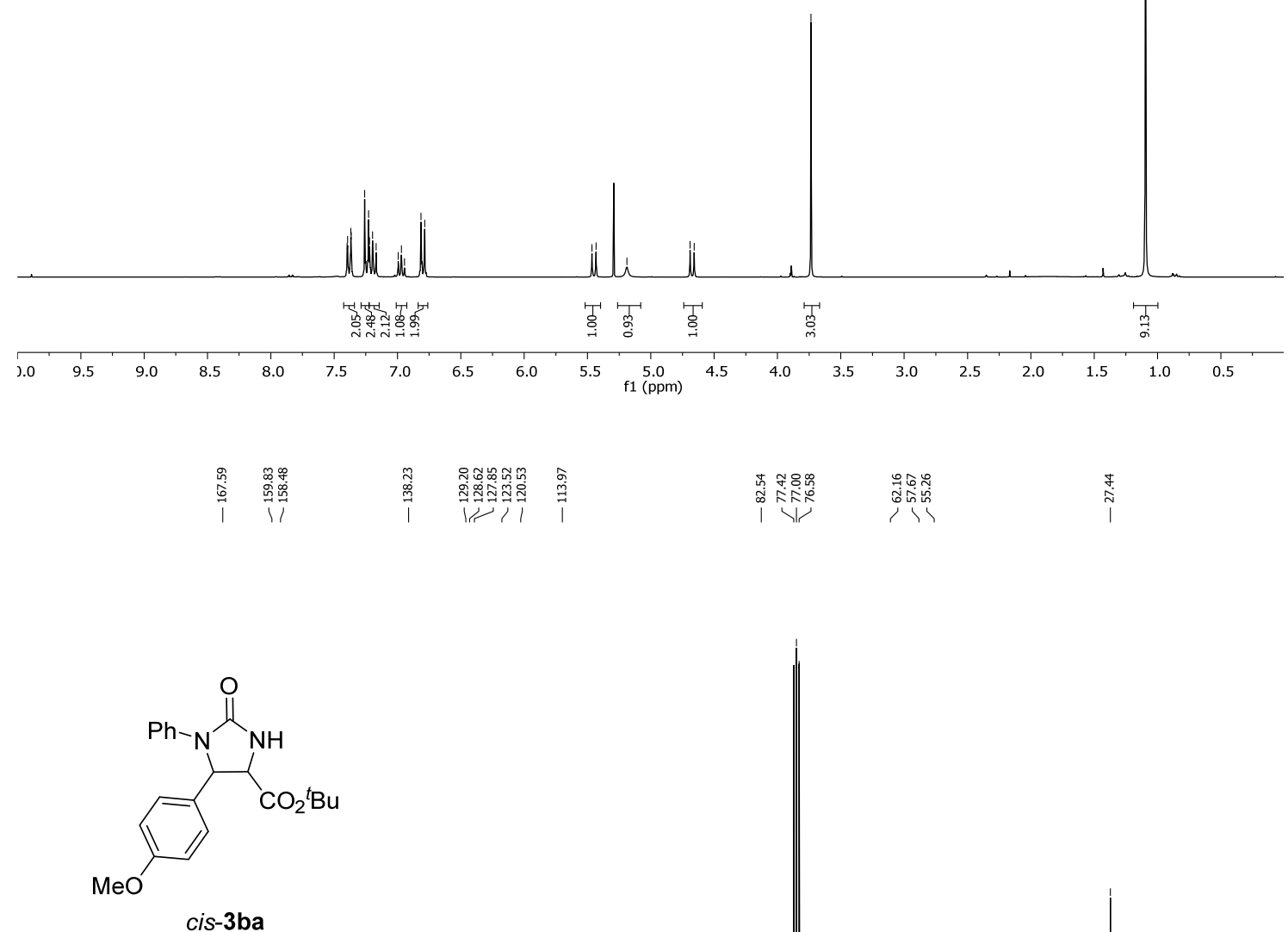

${ }^{13} \mathrm{C} \mathrm{NMR}, \mathrm{CDCl}_{3}, 75 \mathrm{MHz}$

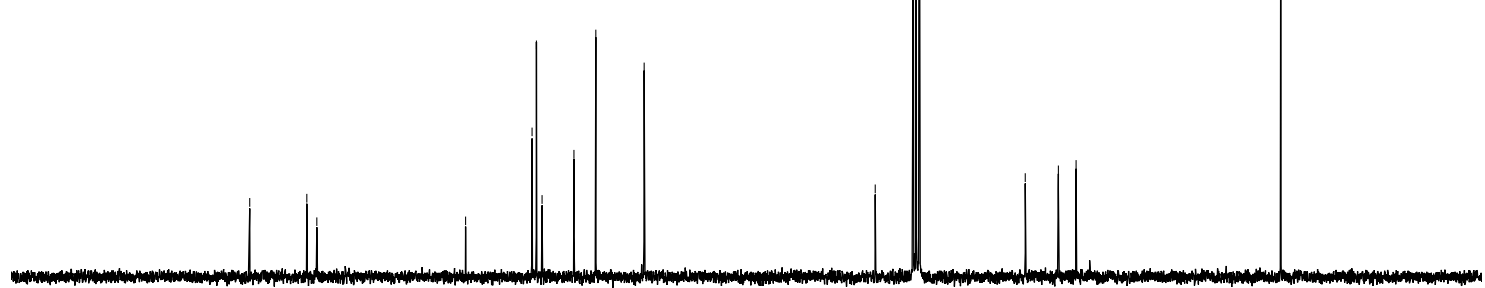

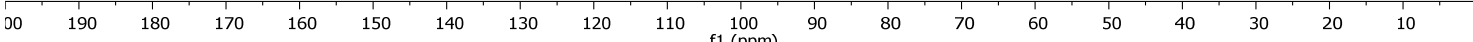




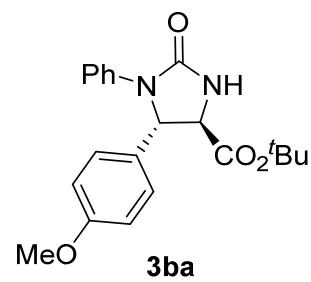

Racemic product (diastereomer mixture)

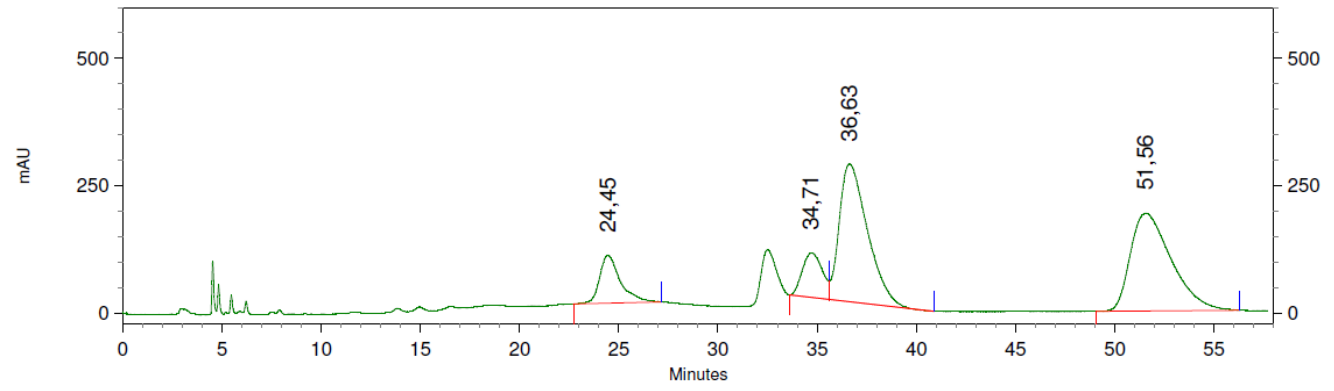

8: $229 \mathrm{~nm}, 4 \mathrm{~nm}$ Results

\begin{tabular}{rrr} 
Retention Time & Area & Area Percent \\
\hline 24,45 & 27385565 & 10,191 \\
34,71 & 24659508 & 9,176 \\
36,63 & 106613724 & 39,673 \\
51,56 & 110070392 & 40,960
\end{tabular}

trans-(4R,5S)-3ba

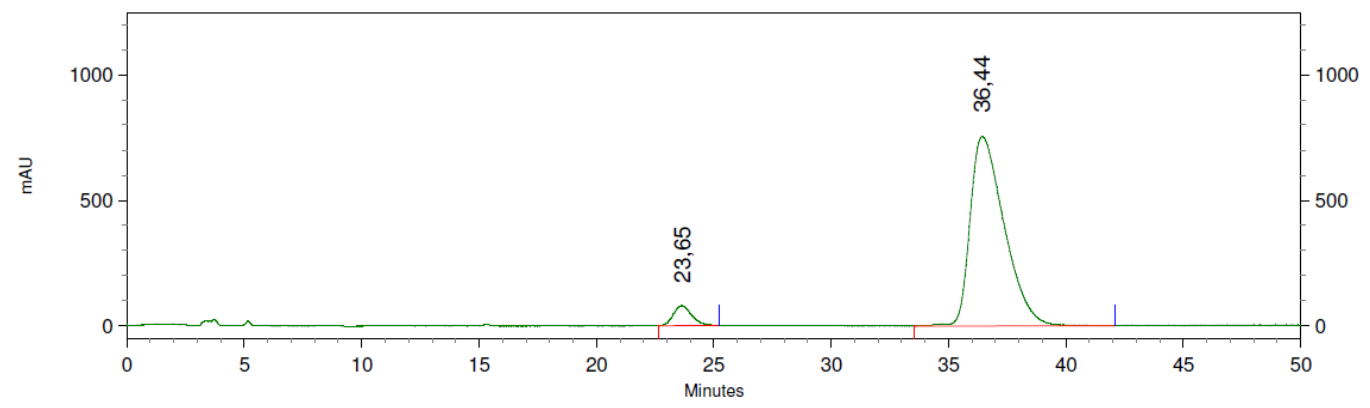

2: $232 \mathrm{~nm}, 4 \mathrm{~nm}$ Results Retention Time 23,65
36,44 Area Percent

\begin{tabular}{rrr} 
Retention Time & Area & Area Percent \\
\hline 23,65 & 17165279 & 5,326 \\
36,44 & 305124181 & 94,674
\end{tabular}

cis-3ba

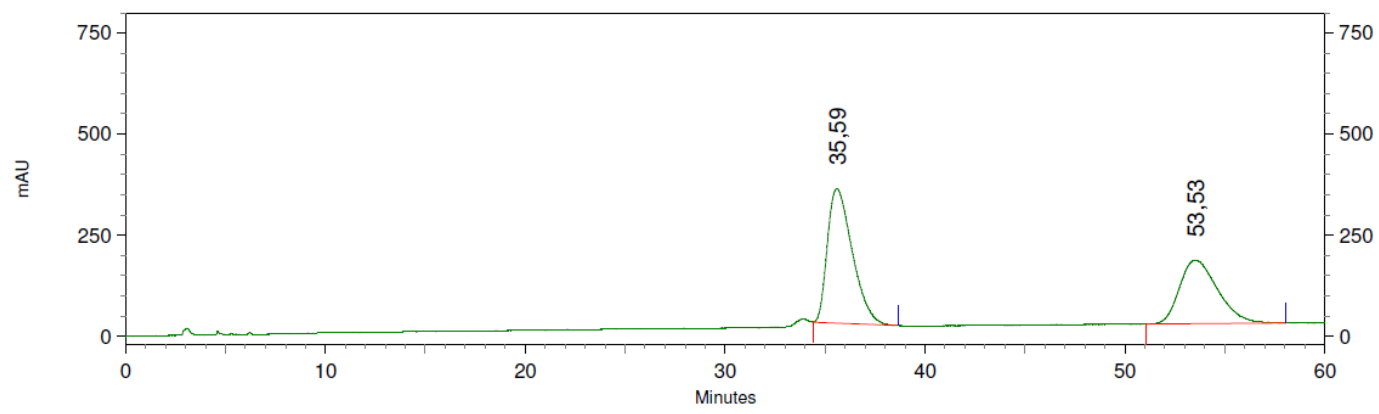

2: $232 \mathrm{~nm}, 4 \mathrm{~nm}$ Results Retention Time 


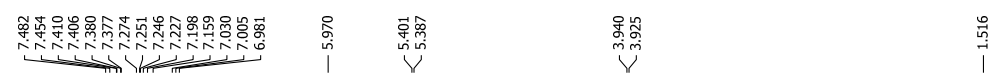

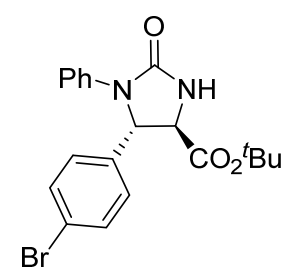

trans-(4R,5S)-3ca

${ }^{1} \mathrm{H} \mathrm{NMR}, \mathrm{CDCl}_{3}, 300 \mathrm{MHz}$

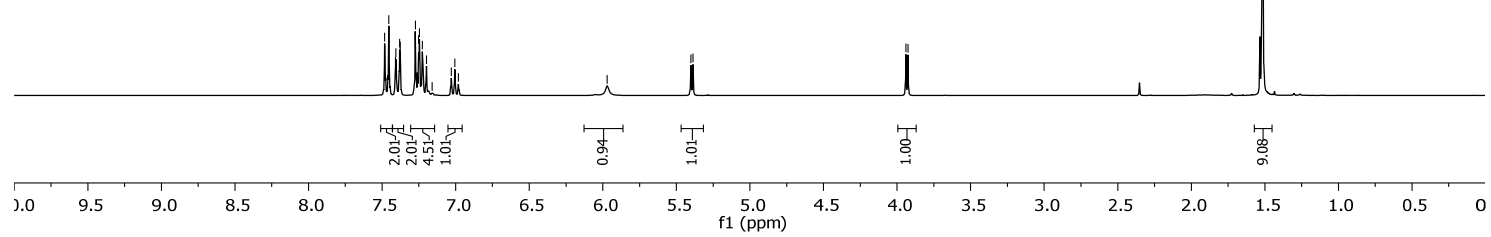

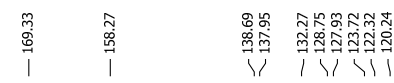

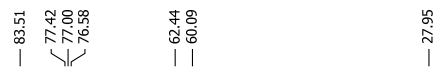

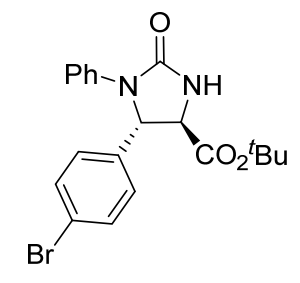

trans-(4R,5S)-3ca

${ }^{13} \mathrm{C} \mathrm{NMR}, \mathrm{CDCl}_{3}, 75 \mathrm{MHz}$

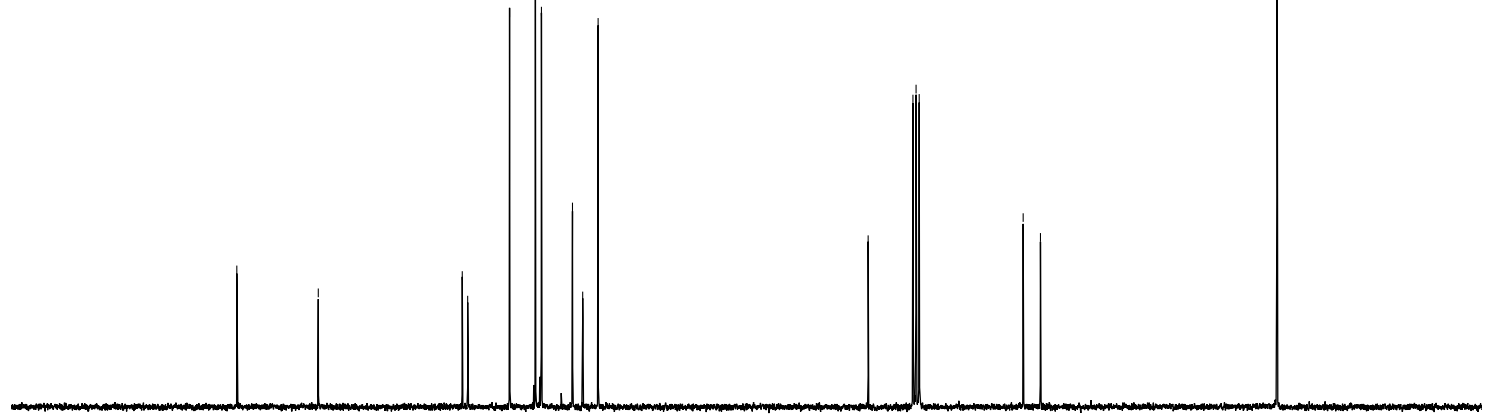

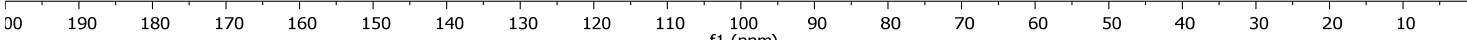




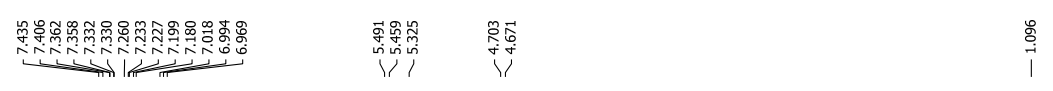

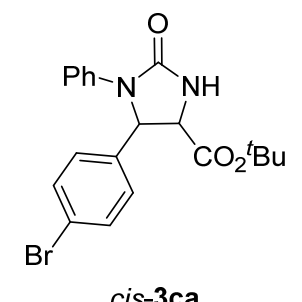

${ }^{1} \mathrm{H} \mathrm{NMR}, \mathrm{CDCl}_{3}, 300 \mathrm{MHz}$

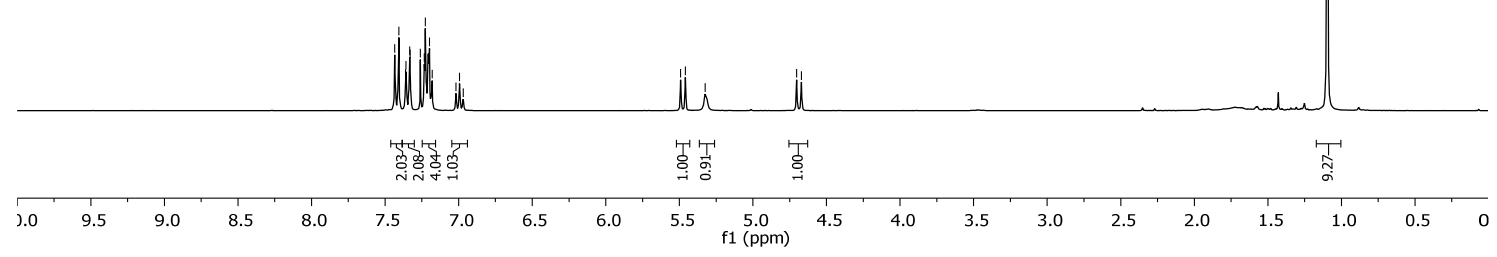

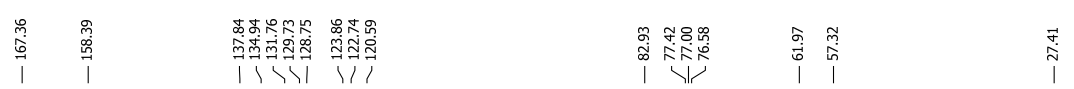

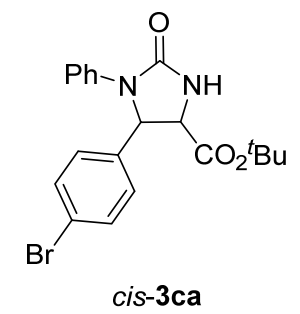

${ }^{13} \mathrm{C} \mathrm{NMR} \mathrm{CDCl}_{3}, 75 \mathrm{MHz}$

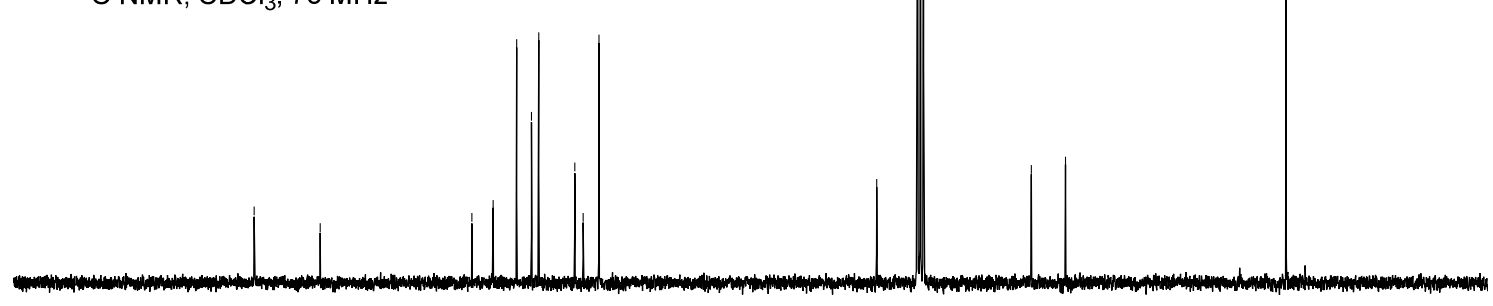

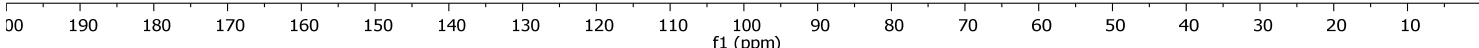




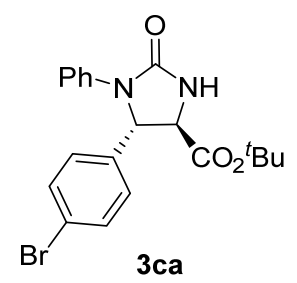

Racemic product (diastereomer mixture)

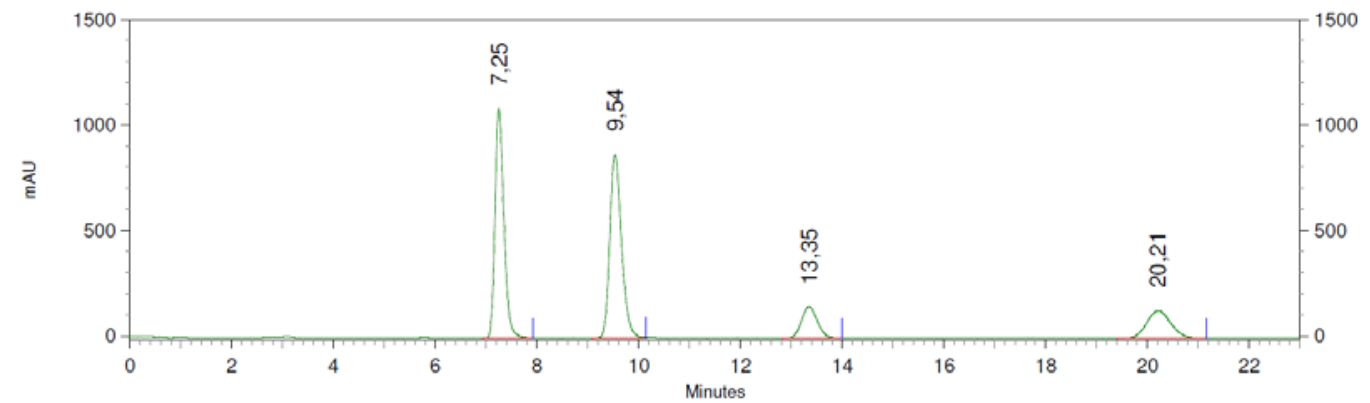

8: $229 \mathrm{~nm}, 4 \mathrm{~nm}$ Results

\begin{tabular}{rrr} 
Retention Time & Area & Area Percent \\
\hline 7,25 & 52830466 & 37,942 \\
9,54 & 55762594 & 40,048 \\
13,35 & 13180970 & 9,466 \\
20,21 & 17467041 & 12,544
\end{tabular}

trans-(4R,5S)-3ca

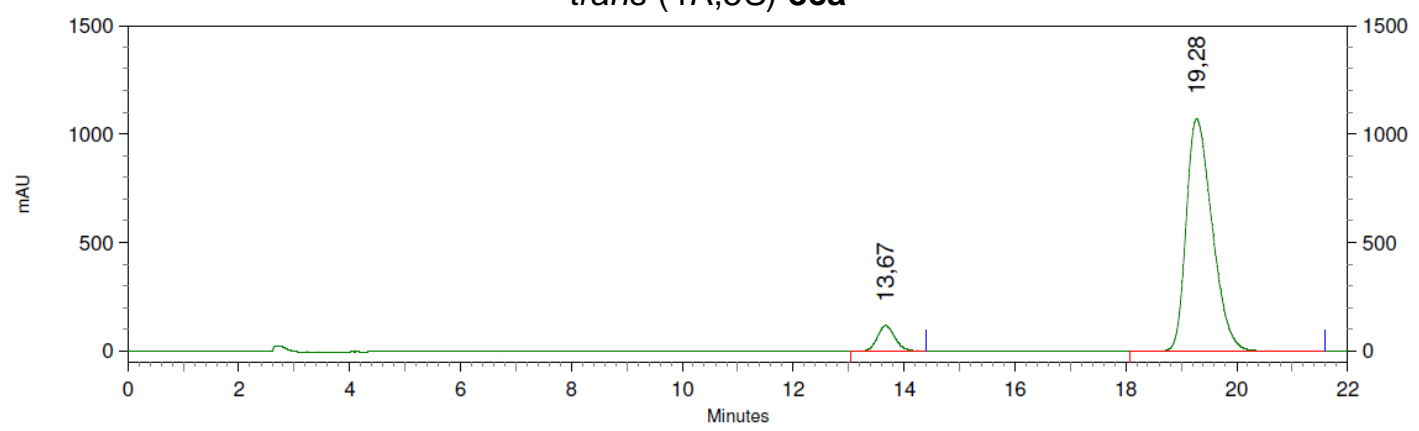

2: $229 \mathrm{~nm}, 4 \mathrm{~nm}$ Results Retention Time

$\begin{array}{rrr}13,67 & \text { Area } & 6,907 \\ 19,28 & 10320379 & 93,093\end{array}$

cis-3ca

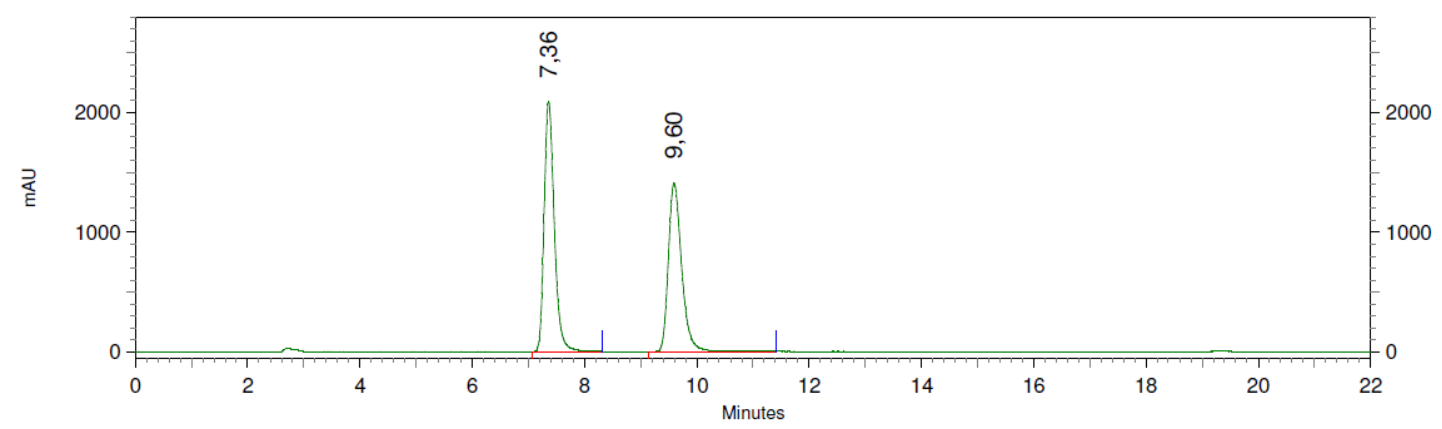

2: $229 \mathrm{~nm}, 4 \mathrm{~nm}$ Results Retention Time

$\begin{array}{rrr}7,36 & 104864558 & 53,027 \\ 9,60 & 92894168 & 46,973\end{array}$




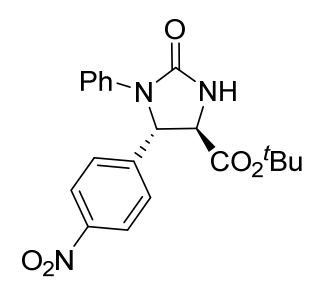

trans-(4R,5S)-3da

${ }^{1} \mathrm{H} \mathrm{NMR}, \mathrm{CDCl}_{3}, 300 \mathrm{MHz}$
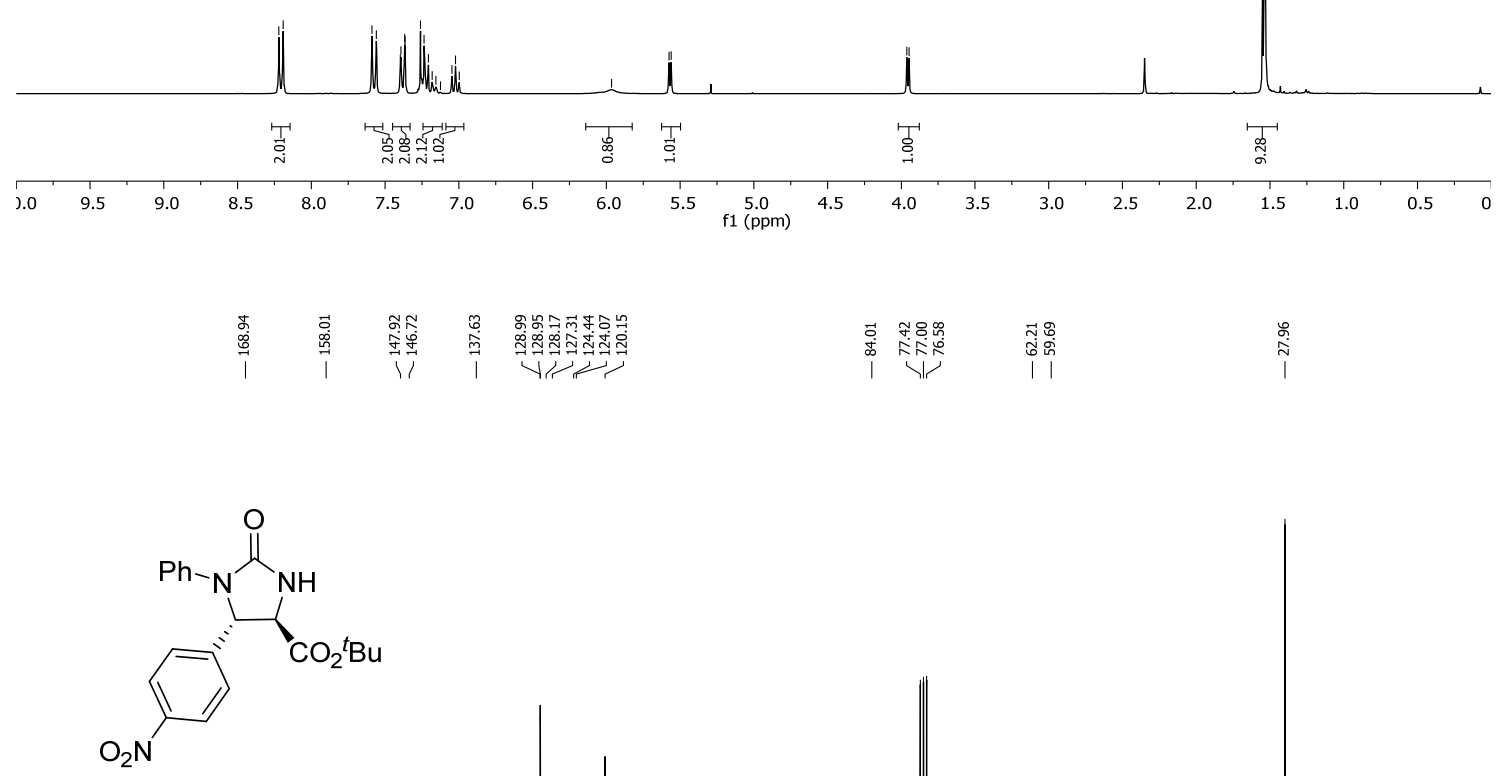

trans-(4R,5S)-3da

${ }^{13} \mathrm{C} \mathrm{NMR}, \mathrm{CDCl}_{3}, 75 \mathrm{MHz}$

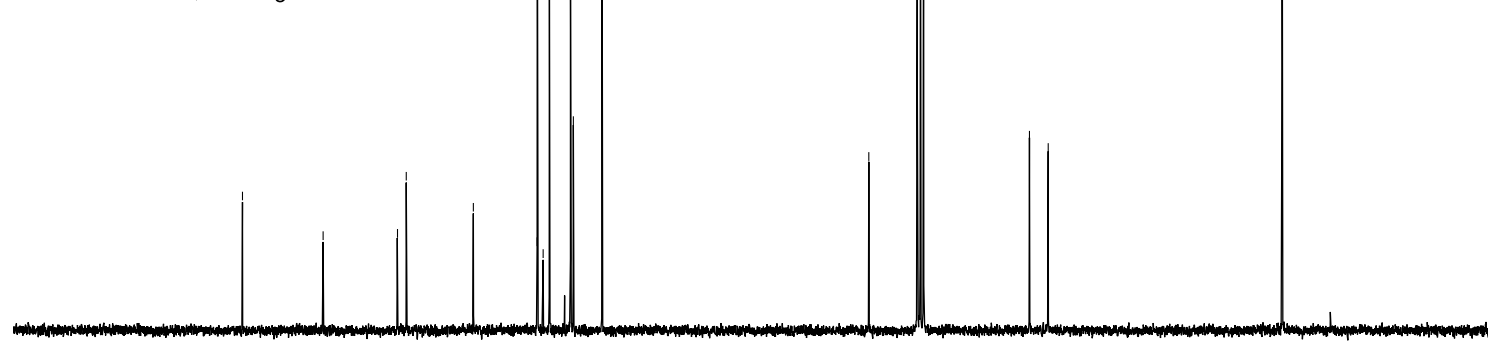

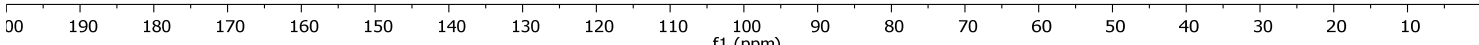




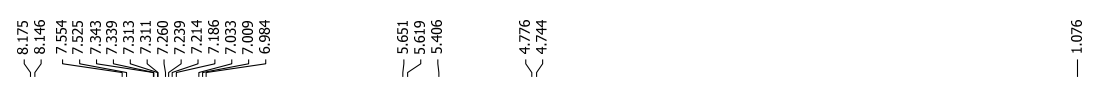

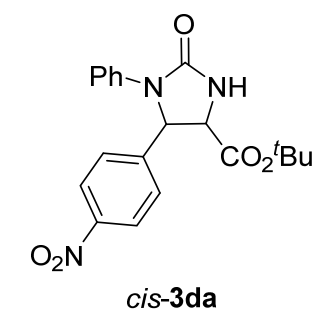

${ }^{1} \mathrm{H} \mathrm{NMR}, \mathrm{CDCl}_{3}, 300 \mathrm{MHz}$
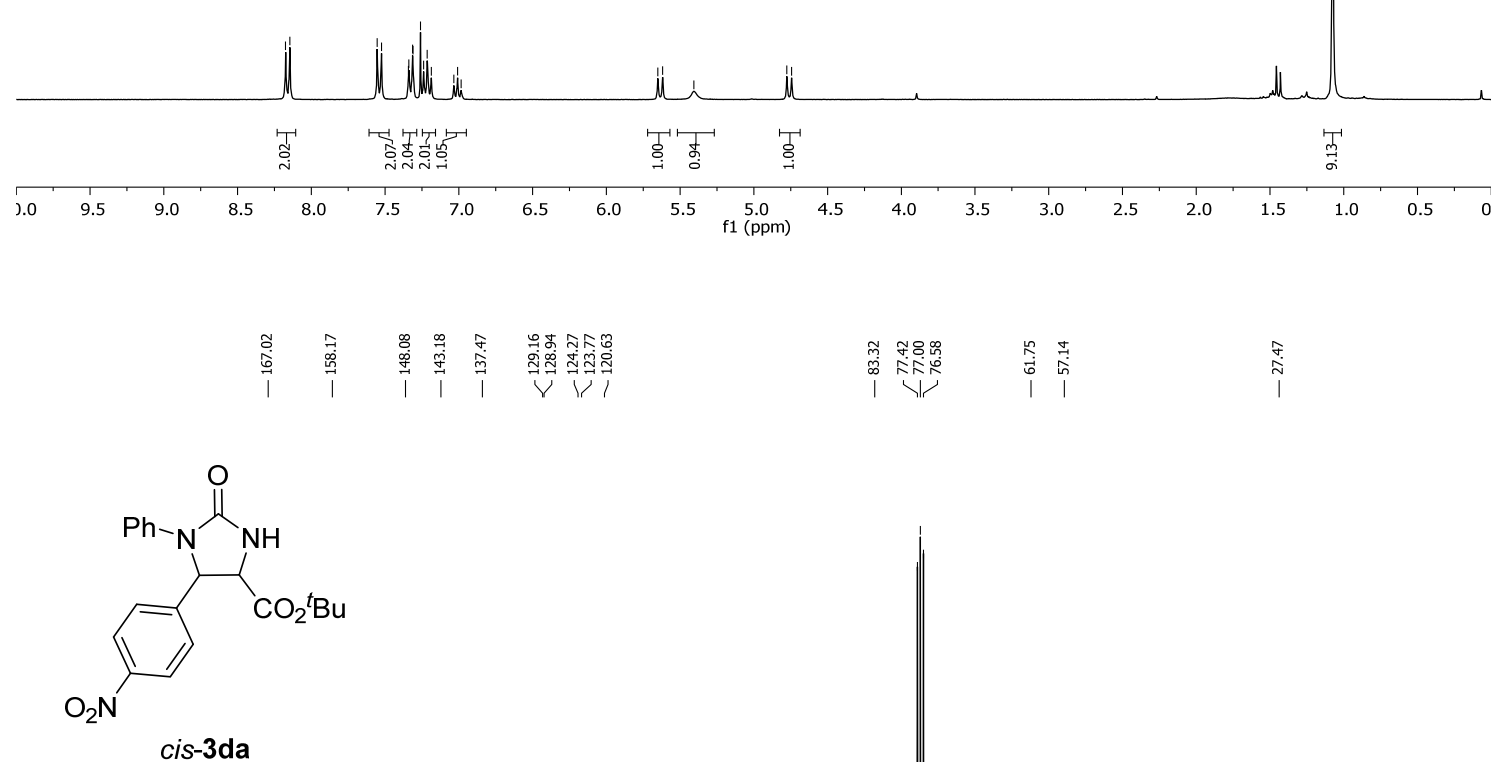

${ }^{13} \mathrm{C} \mathrm{NMR}, \mathrm{CDCl}_{3}, 75 \mathrm{MHz}$

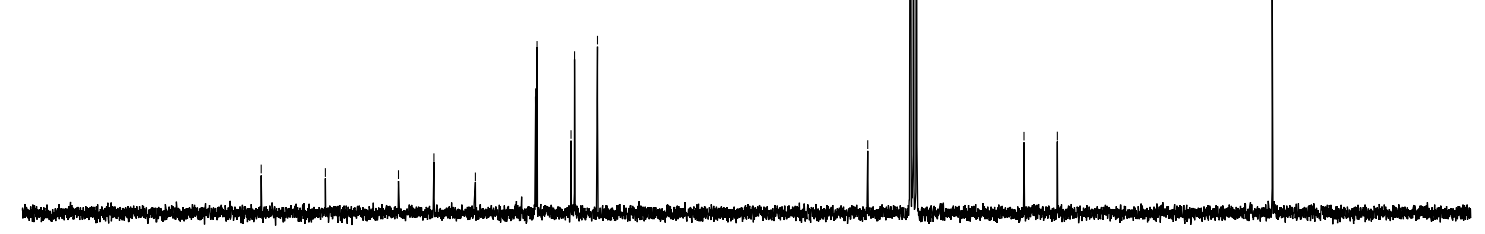

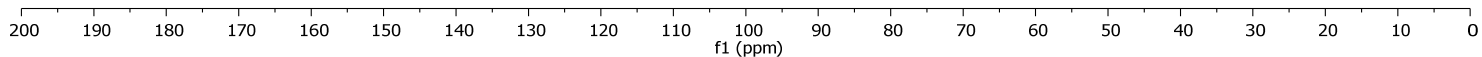



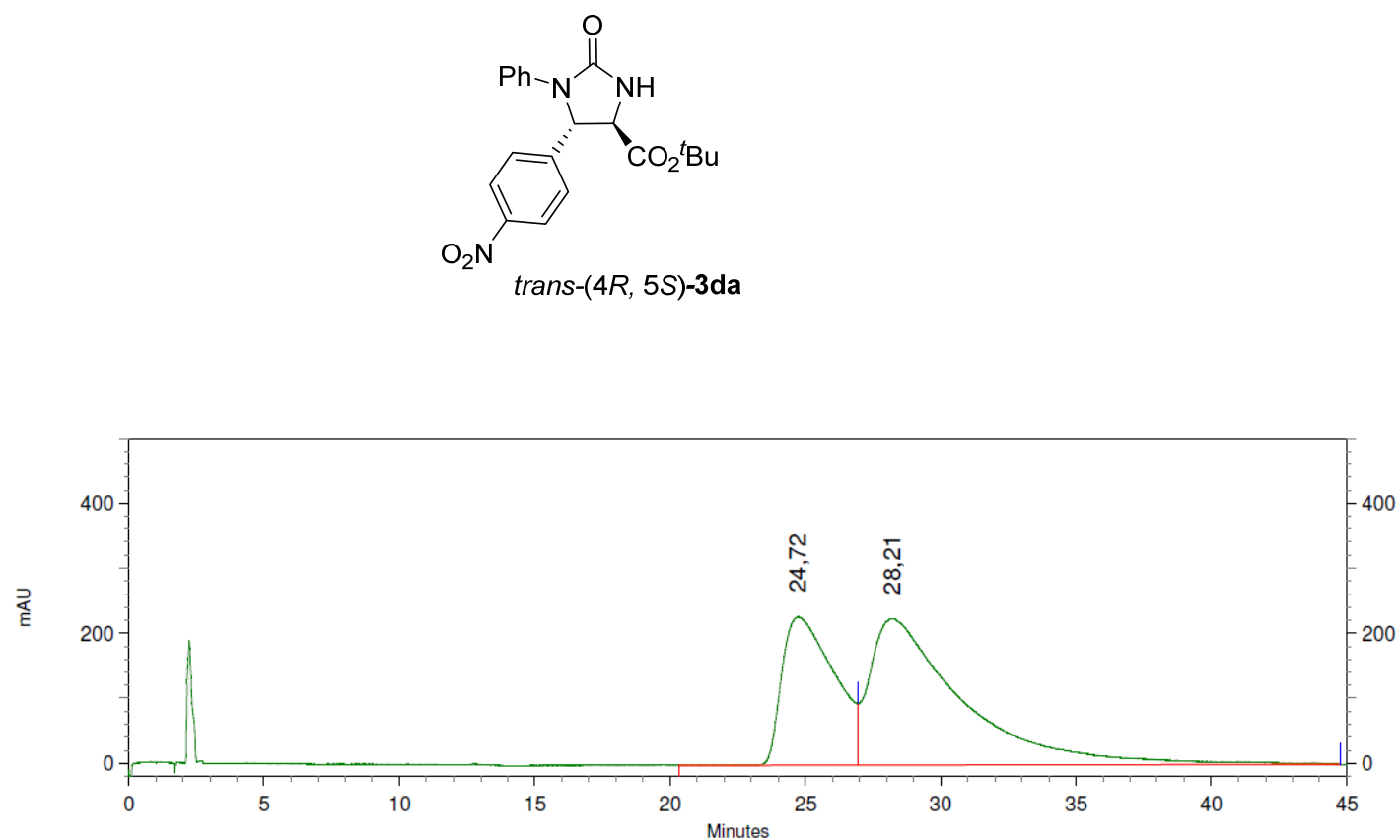

1: $242 \mathrm{~nm}, 4 \mathrm{~nm}$ Results Retention Time Area Area Percent 24,72 122620419 36,444

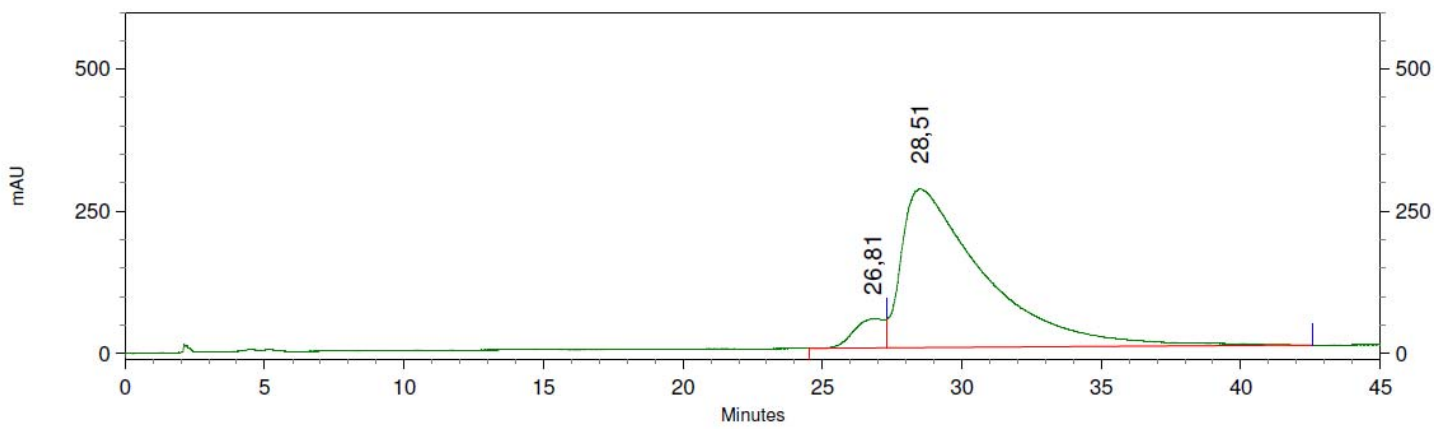

1: $242 \mathrm{~nm}, 4 \mathrm{~nm}$ Results Retention Time

Area Area Percent

$\begin{array}{rrr}26,81 & 15468797 & 6,302 \\ 28,51 & 229999260 & 93,698\end{array}$



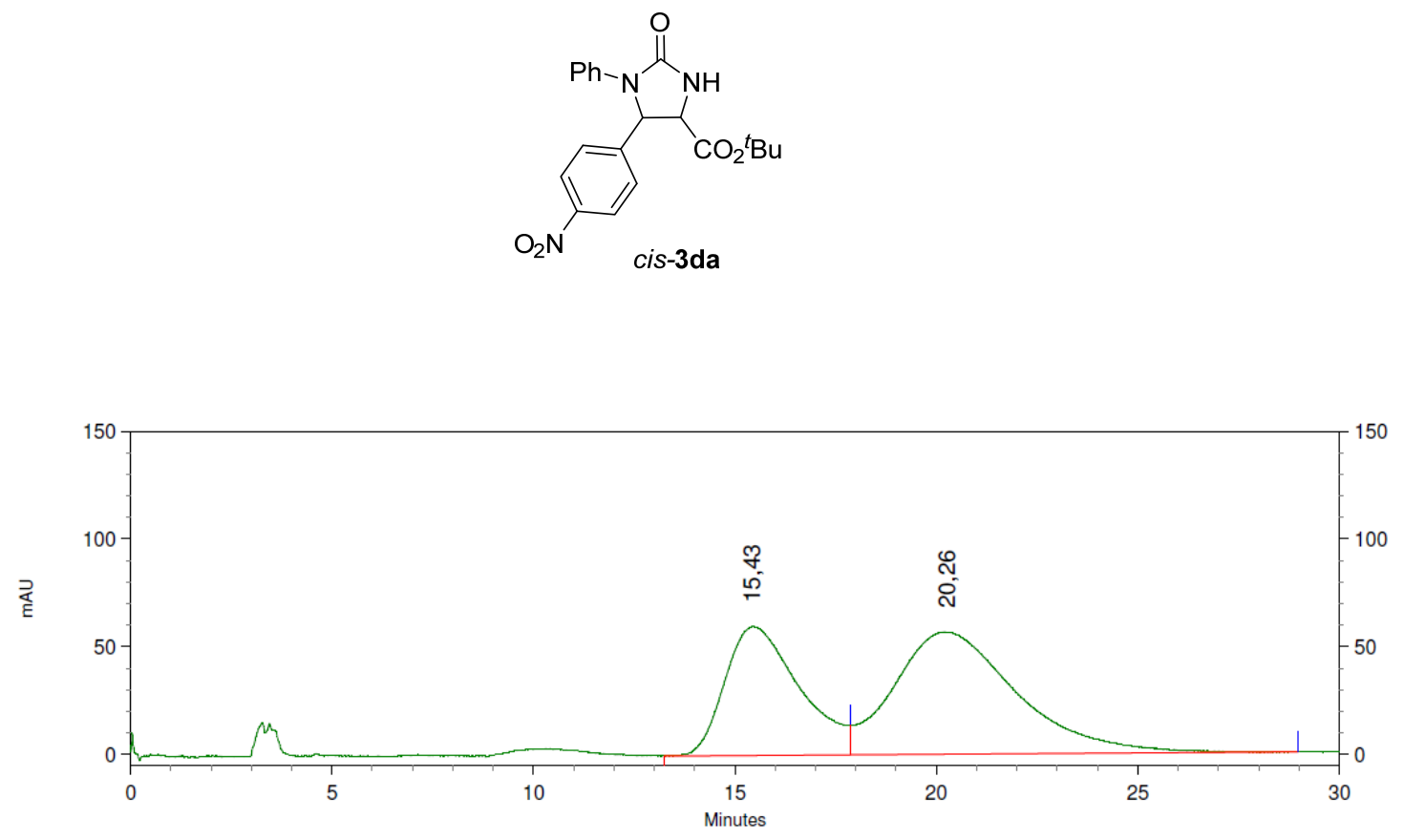

6: 242 nm, 4 nm Results Retention Time

15,43 Area 30686553 Area Percent 20,26 47807668 39,094
60,906

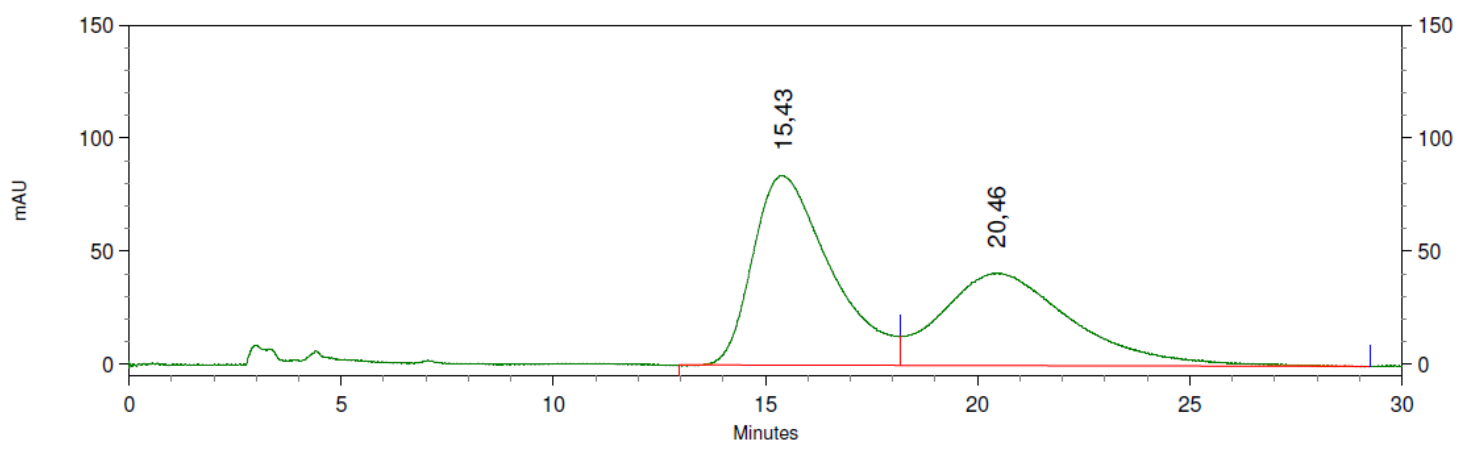

6: 242 nm, 4 nm Results Retention Time Area Area Percent 15,43 42776248 55,149 20,46 34788613

44,851 


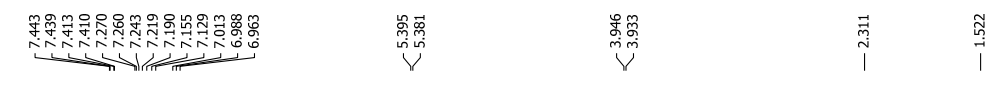

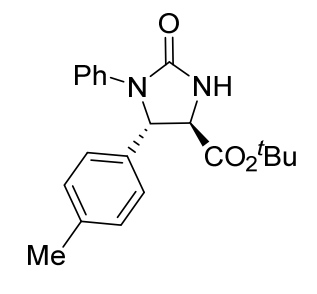

trans-(4R,5S)-3ea

${ }^{1} \mathrm{H} \mathrm{NMR}, \mathrm{CDCl}_{3}, 300 \mathrm{MHz}$

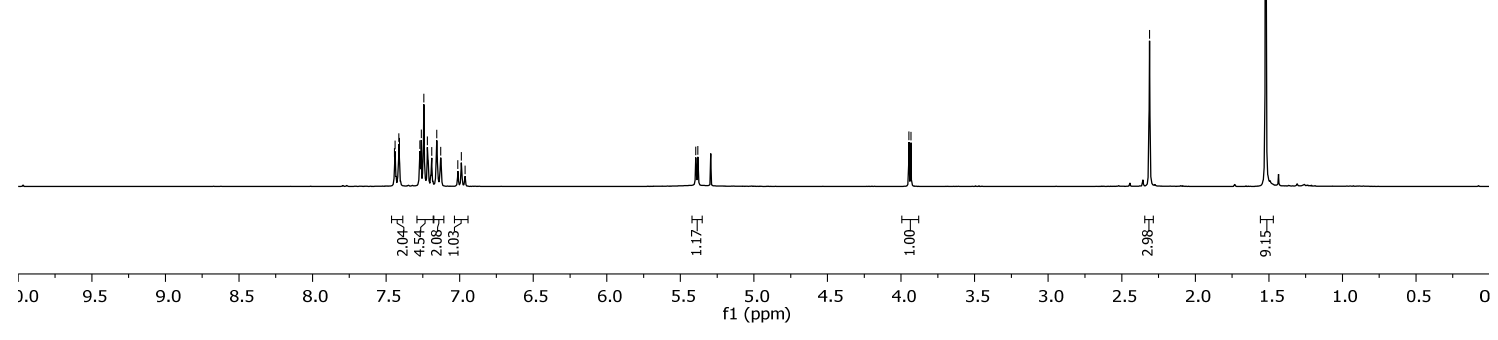

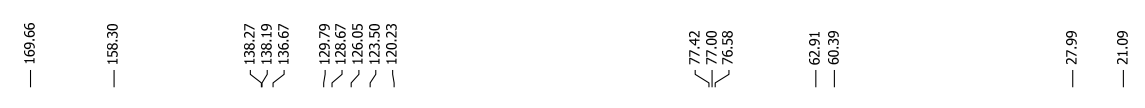

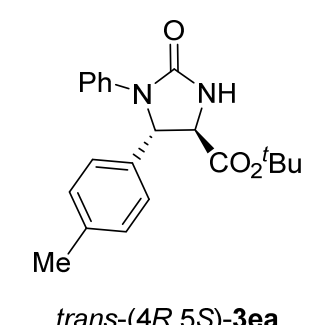

${ }^{13} \mathrm{C} \mathrm{NMR}, \mathrm{CDCl}_{3}, 75 \mathrm{MHz}$

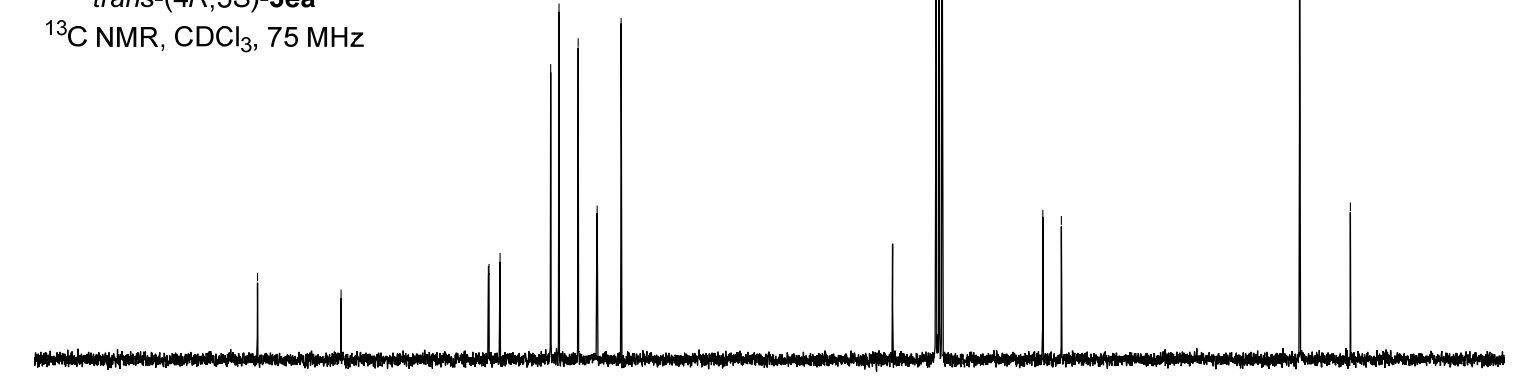

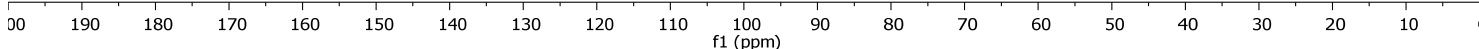




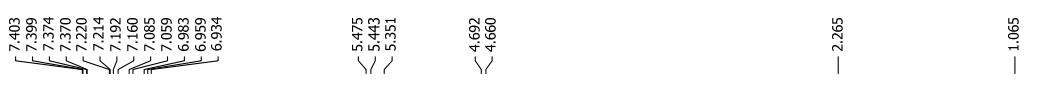

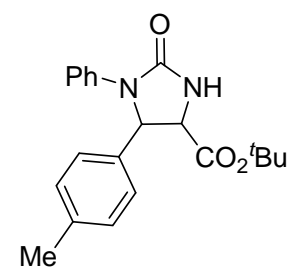

cis-3ea

${ }^{1} \mathrm{H} \mathrm{NMR}, \mathrm{CDCl}_{3}, 300 \mathrm{MHz}$

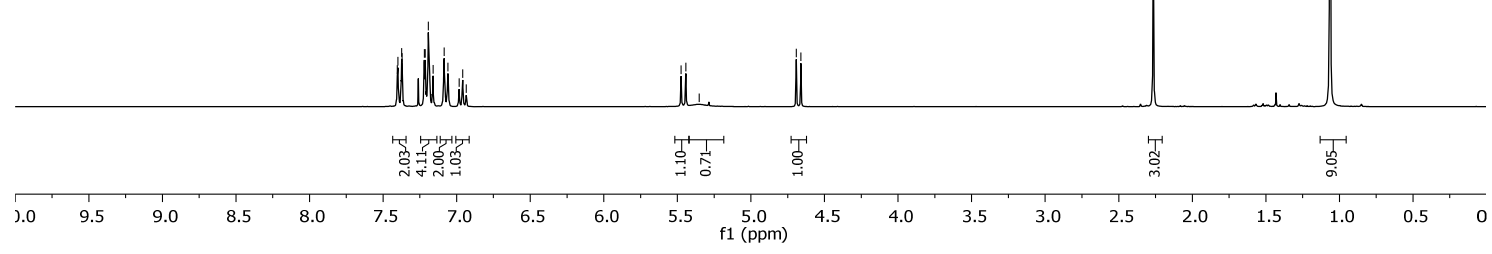

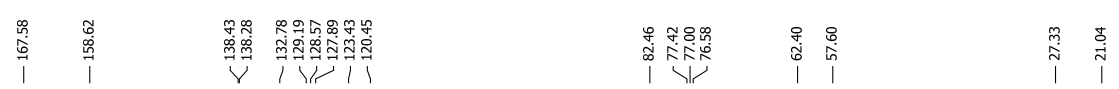

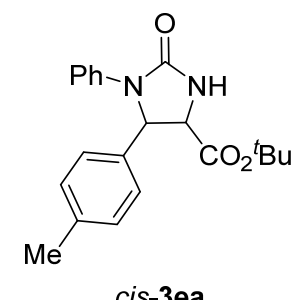

${ }^{13} \mathrm{C} \mathrm{NMR}, \mathrm{CDCl}_{3}, 75 \mathrm{MHz}$

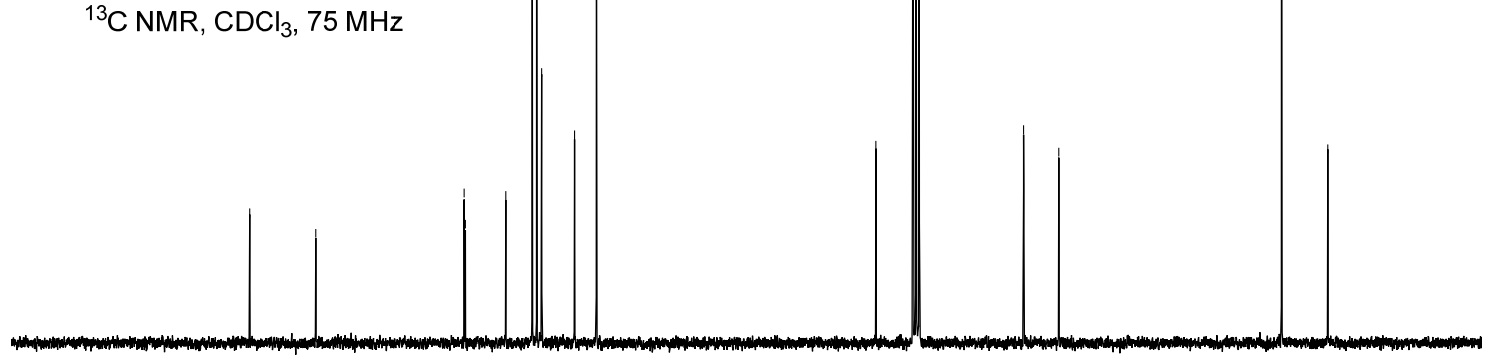

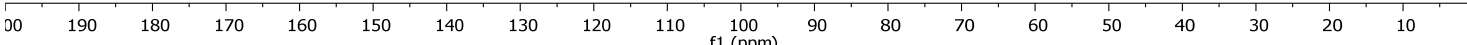




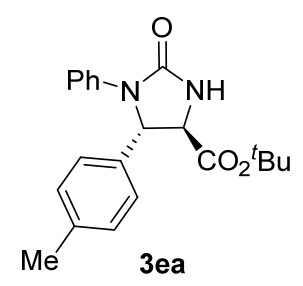

Racemic product (diastereomer mixture)

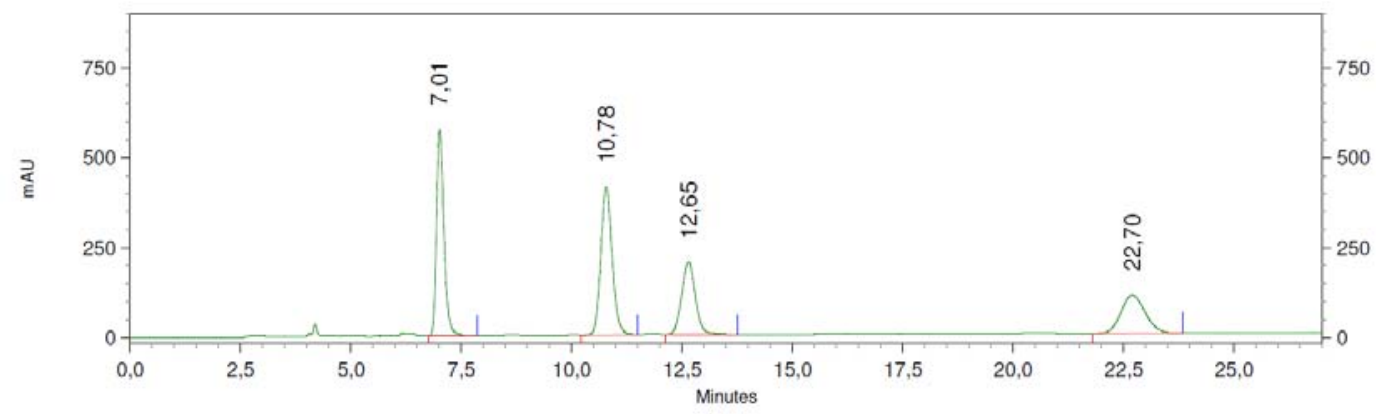

2: $241 \mathrm{~nm}, 4 \mathrm{~nm}$ Results

\begin{tabular}{rrr} 
Retention Time & Area & Area Percent \\
\hline 7,01 & 26725257 & 29,770 \\
10,78 & 29350454 & 32,695 \\
12,65 & 17537558 & 19,536 \\
22,70 & 16158279 & 17,999
\end{tabular}

trans-(4R,5S)-3ea

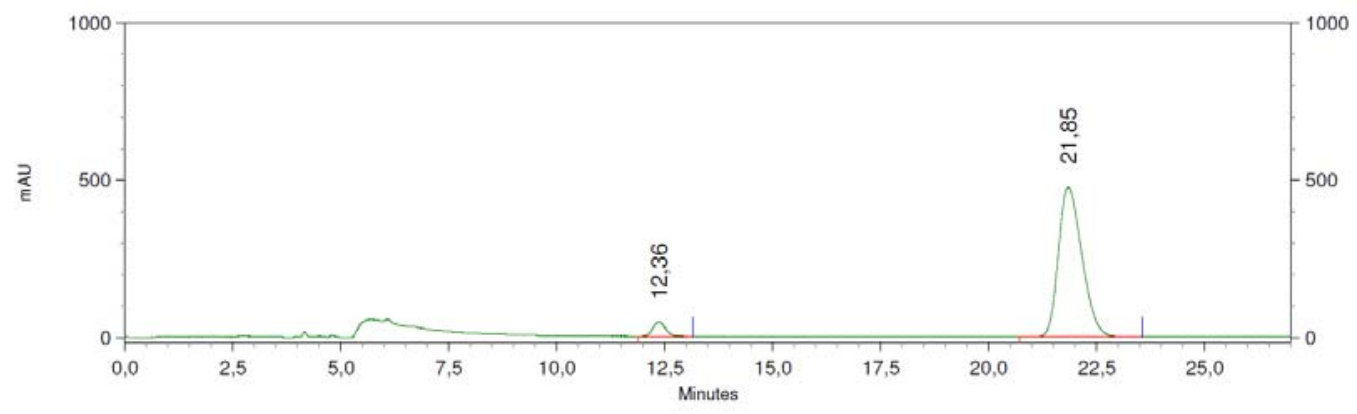

2: $241 \mathrm{~nm}, 4 \mathrm{~nm}$ Results

\begin{tabular}{|c|c|c|}
\hline Retention Time & Area & Area Percent \\
\hline $\begin{array}{l}12,36 \\
21,85\end{array}$ & $\begin{array}{r}3909678 \\
72327678\end{array}$ & $\begin{array}{r}5,128 \\
94,872\end{array}$ \\
\hline
\end{tabular}

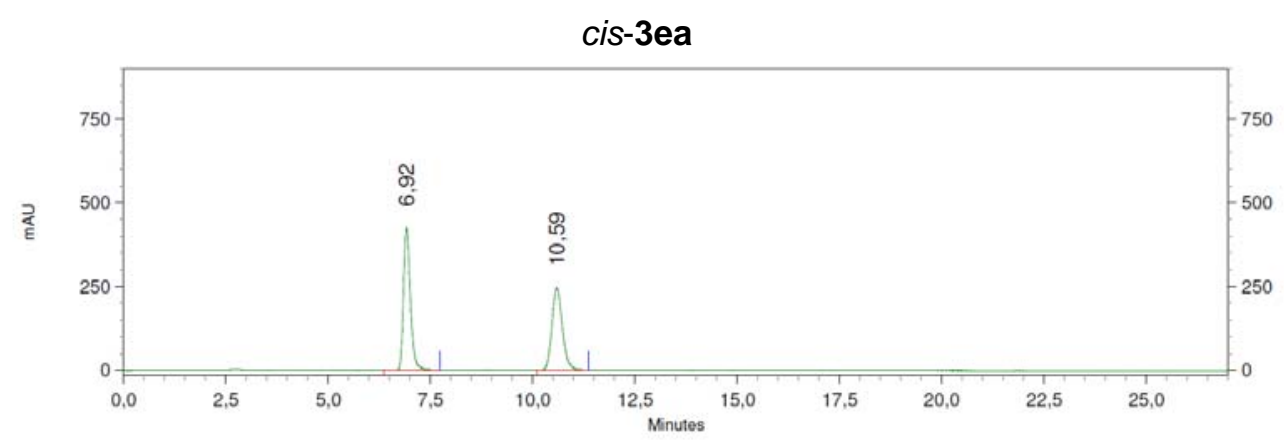

2: $241 \mathrm{~nm}, 4 \mathrm{~nm}$ Results Retention Time

\begin{tabular}{rrr} 
Area & Area Percent \\
\hline 6,92 & 20013869 & 53,473 \\
10,59 & 17413966 & 46,527
\end{tabular}




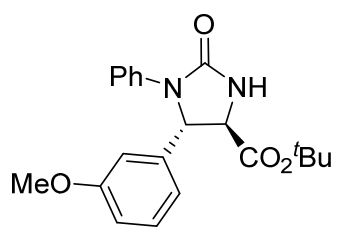

trans-(4R,5S)-3fa

${ }^{1} \mathrm{H} \mathrm{NMR}, \mathrm{CDCl}_{3}, 300 \mathrm{MHz}$

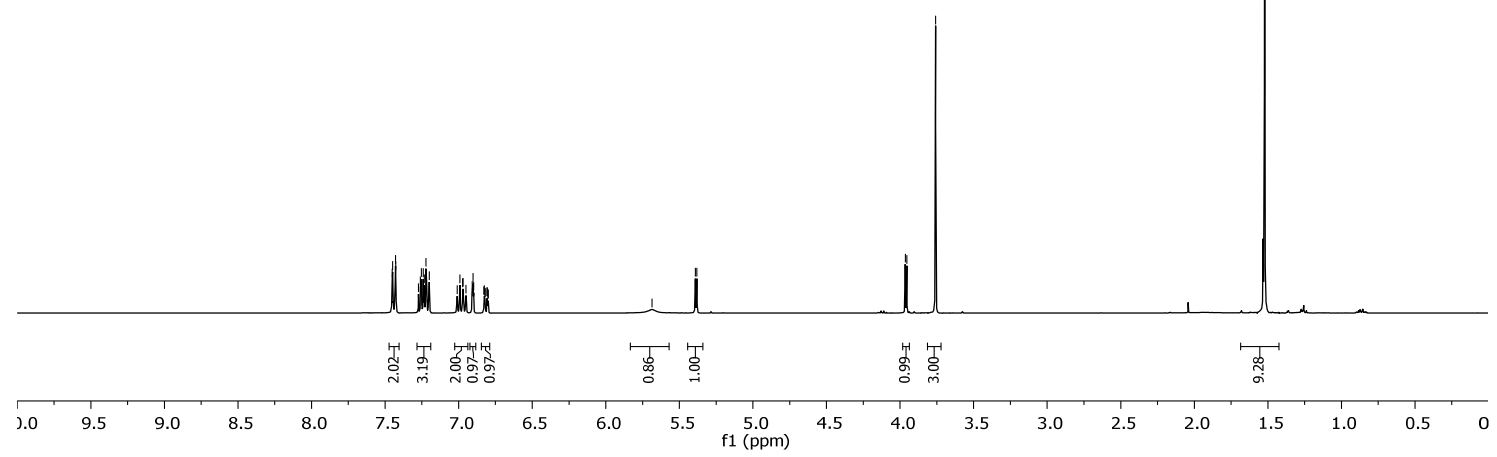

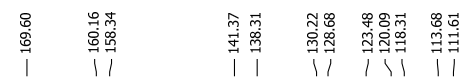

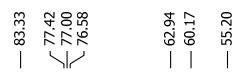<smiles>CCOC(=O)C1NC(=O)N(c2ccccc2)[C@H]1c1cccc(OC)c1</smiles>

trans-(4R,5S)-3fa

${ }^{13} \mathrm{C} \mathrm{NMR}, \mathrm{CDCl}_{3}, 75 \mathrm{MHz}$
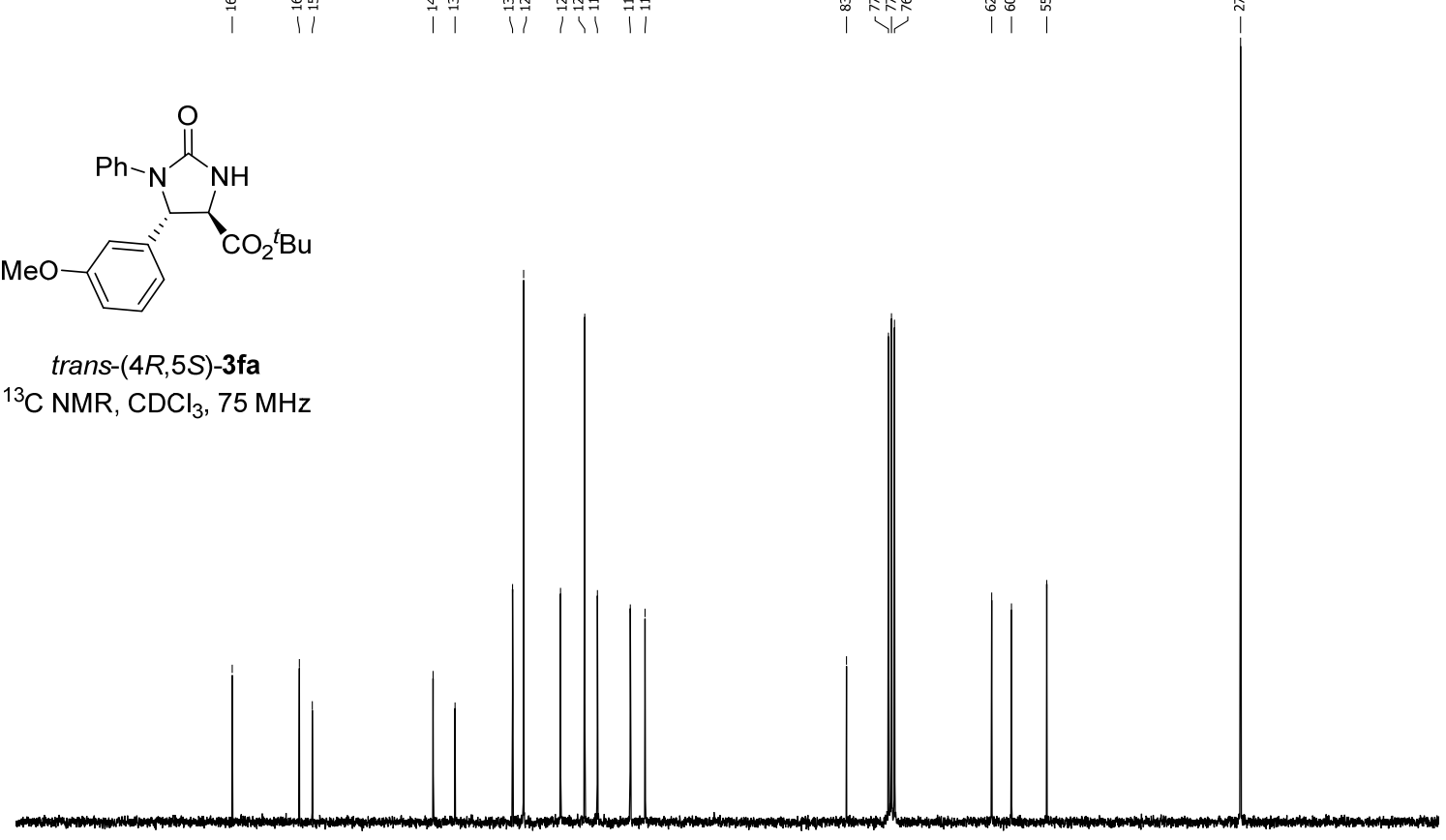

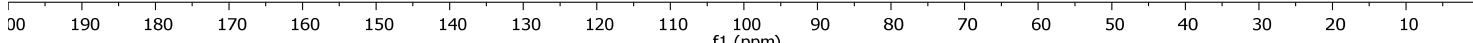




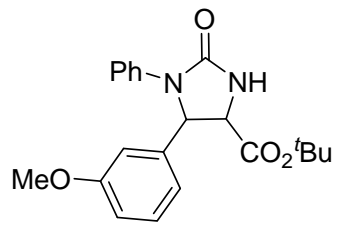

cis-3fa

${ }^{1} \mathrm{H} \mathrm{NMR}, \mathrm{CDCl}_{3}, 300 \mathrm{MHz}$

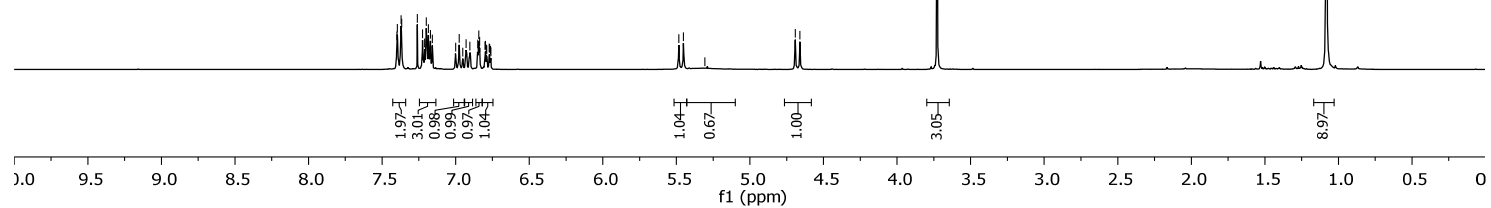

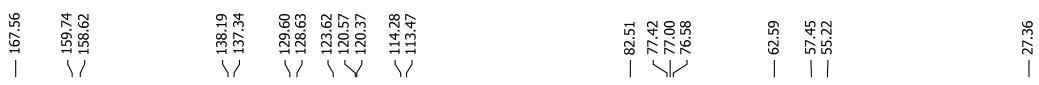

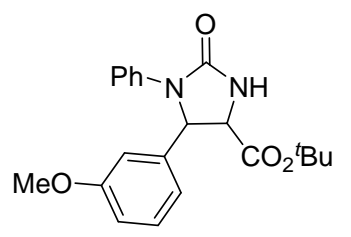

cis-3fa

${ }^{13} \mathrm{C} \mathrm{NMR}, \mathrm{CDCl}_{3}, 75 \mathrm{MHz}$

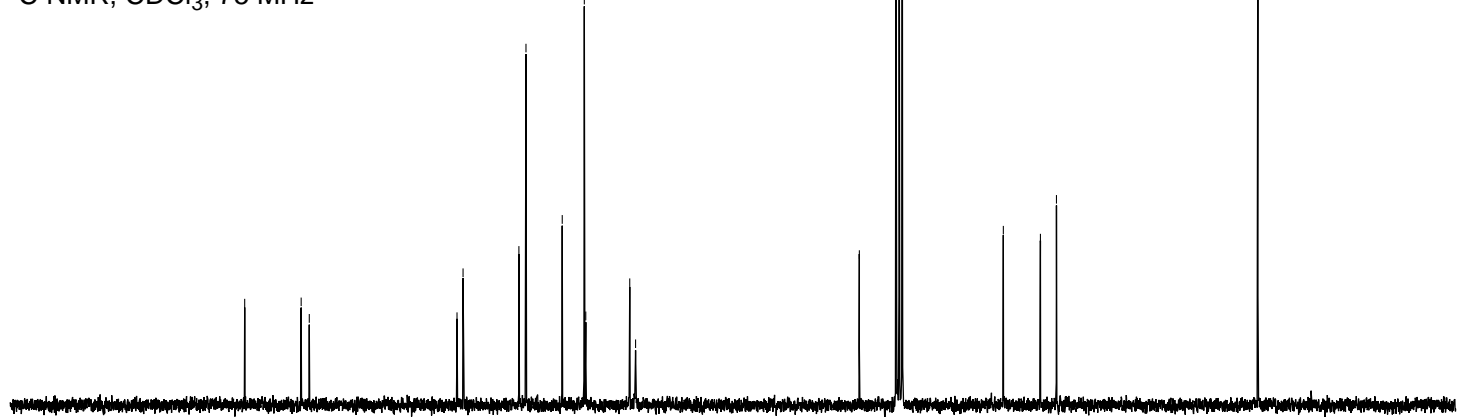

$\begin{array}{lllllllllllllllllllll}200 & 190 & 180 & 170 & 160 & 150 & 140 & 130 & 120 & 110 & 100 & 10 & 10 & 70 & 60 & 50 & 40 & 30 & 20 & 10 & 0\end{array}$ 


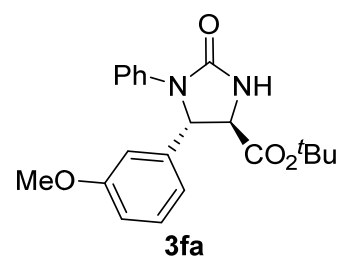

Racemic product (diastereomer mixture)

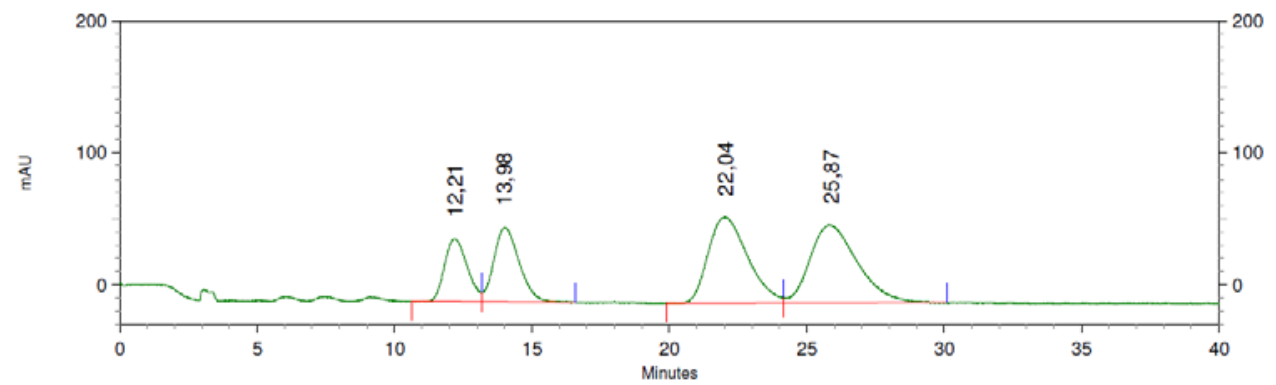

14: $242 \mathrm{~nm}, 4 \mathrm{~nm}$ Results

\begin{tabular}{rrr} 
Retention Time & Area & Area Percent \\
\hline 12,21 & 10978665 & 13,801 \\
13,98 & 14564908 & 18,309 \\
22,04 & 25902138 & 32,561 \\
25,87 & 28104928 & 35,330
\end{tabular}

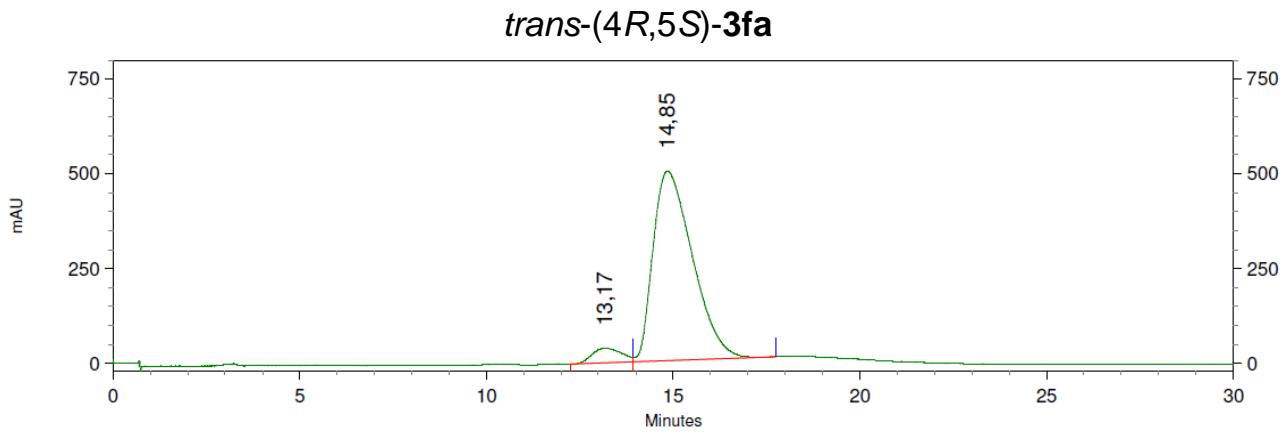

14: $242 \mathrm{~nm}, 4 \mathrm{~nm}$

Results

\begin{tabular}{rrrr} 
Retention Time & Area & Area Percent \\
\hline 13,17 & 8402917 & 5,421 \\
14,85 & 146605488 & 94,579
\end{tabular}

cis-3fa

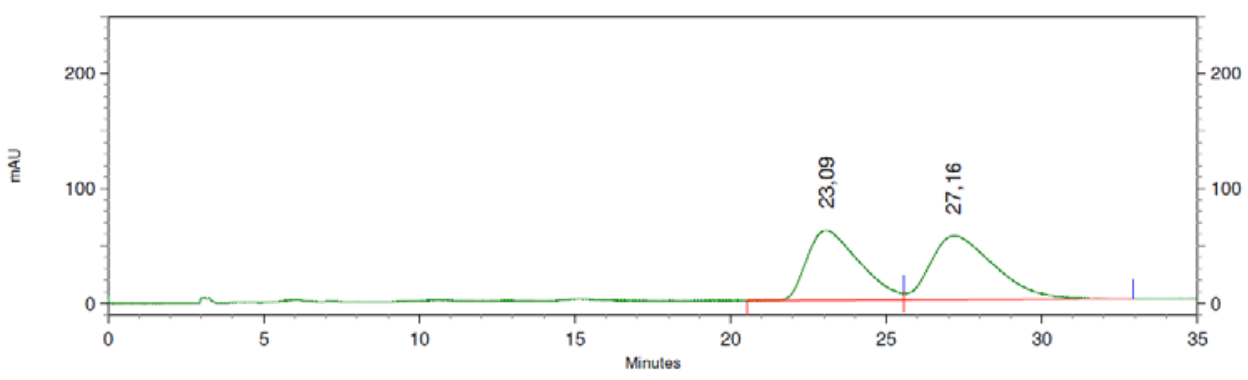

14: $242 \mathrm{~nm}, 4 \mathrm{~nm}$ Results 


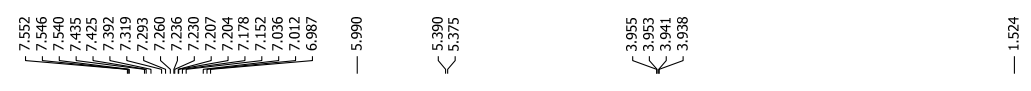

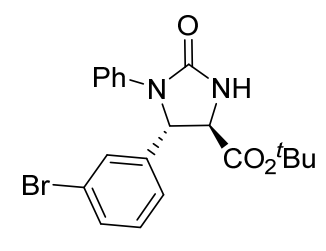

trans-(4R,5S)-3ga

${ }^{1} \mathrm{H} \mathrm{NMR}, \mathrm{CDCl}_{3}, 300 \mathrm{MHz}$

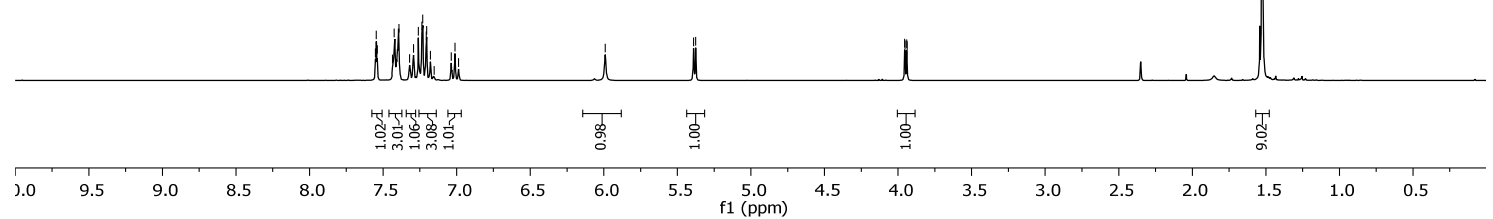

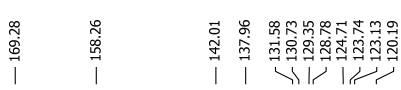

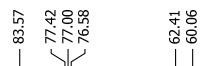

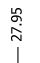<smiles>CCCOC(=O)C1NC(=O)N(c2ccccc2)[C@H]1c1cccc(Br)c1</smiles>

trans-(4R,5S)-3ga

${ }^{13} \mathrm{C} \mathrm{NMR}, \mathrm{CDCl}_{3}, 75 \mathrm{MHz}$

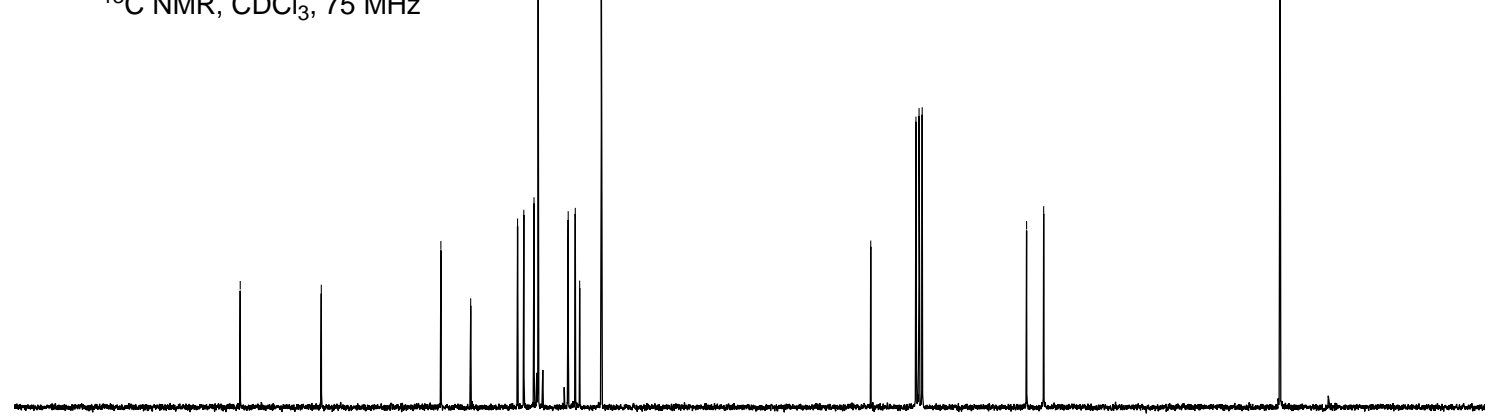

$\begin{array}{lllllllllll}\text { DO } & 190 & 180 & 170 & 160 & 150 & 140 & 130 & 120 & 110 & \begin{array}{l}100 \\ \mathrm{f} 1(\mathrm{ppm})\end{array}\end{array}$ 

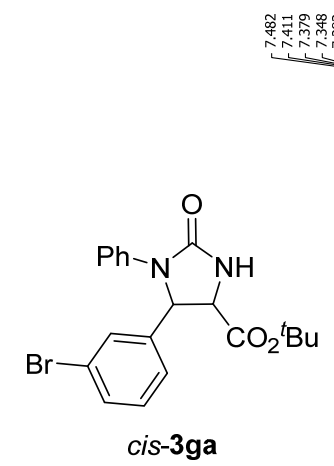

${ }^{1} \mathrm{H} \mathrm{NMR}, \mathrm{CDCl}_{3}, 300 \mathrm{MHz}$

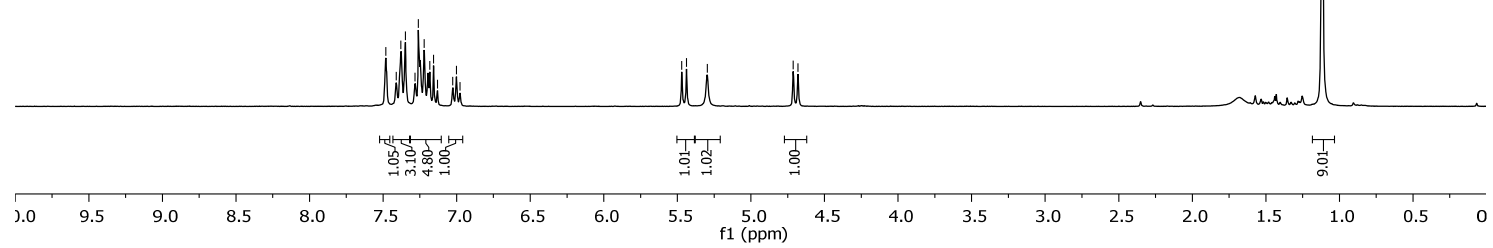

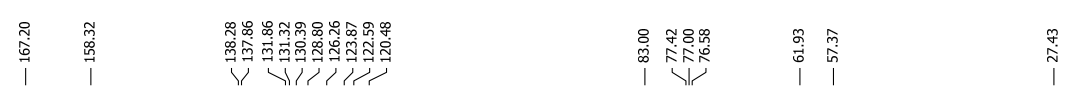

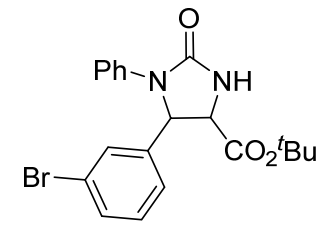

cis-3ga

${ }^{13} \mathrm{C} \mathrm{NMR,} \mathrm{CDCl}_{3}, 75 \mathrm{MHz}$

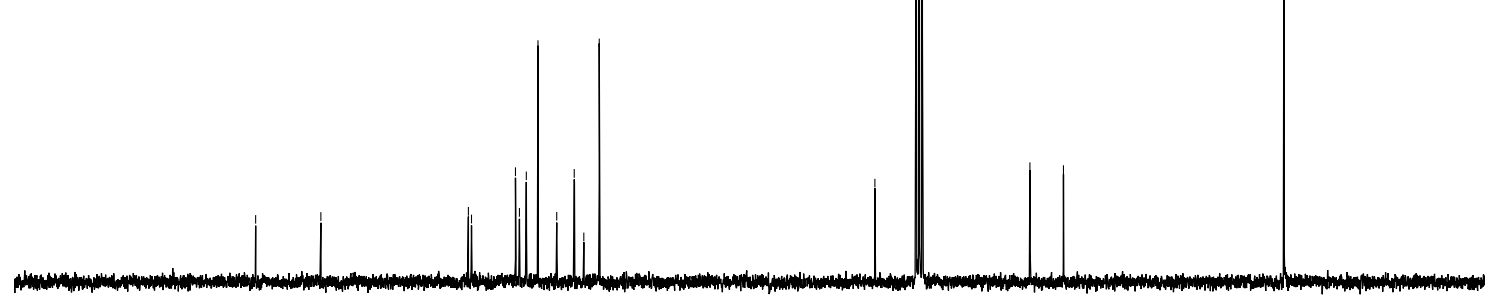

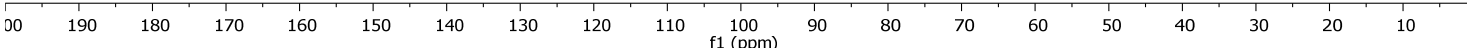




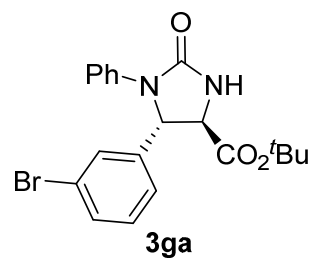

Racemic product (diastereomer mixture)

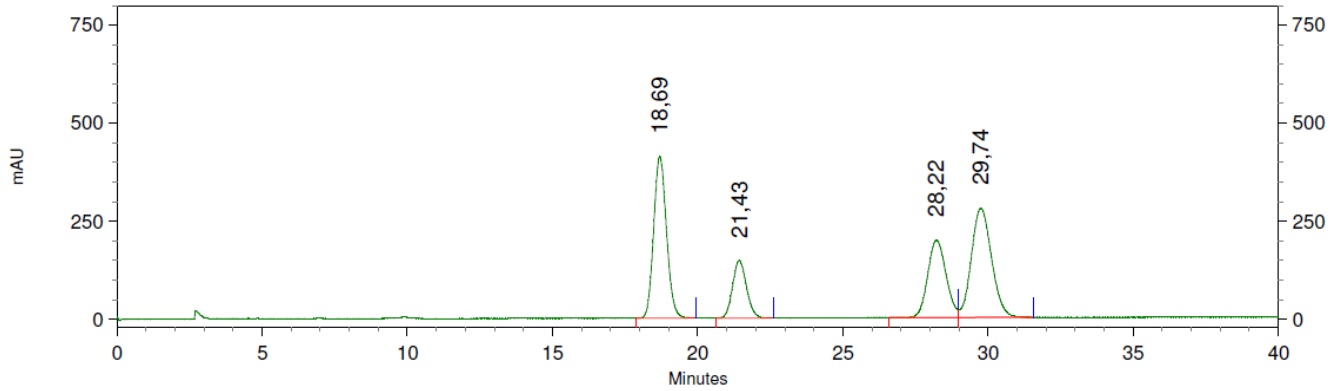

18: $239 \mathrm{~nm}, 4 \mathrm{~nm}$ Results

Retention Time

$\begin{array}{lll}18,69 & 50788647 & 31,999 \\ 21,43 & 19754975 & 12,447 \\ 28,22 & 34489360 & 21,730 \\ 29,74 & 53684185 & 33,824\end{array}$

trans-(4R,5S)-3ga

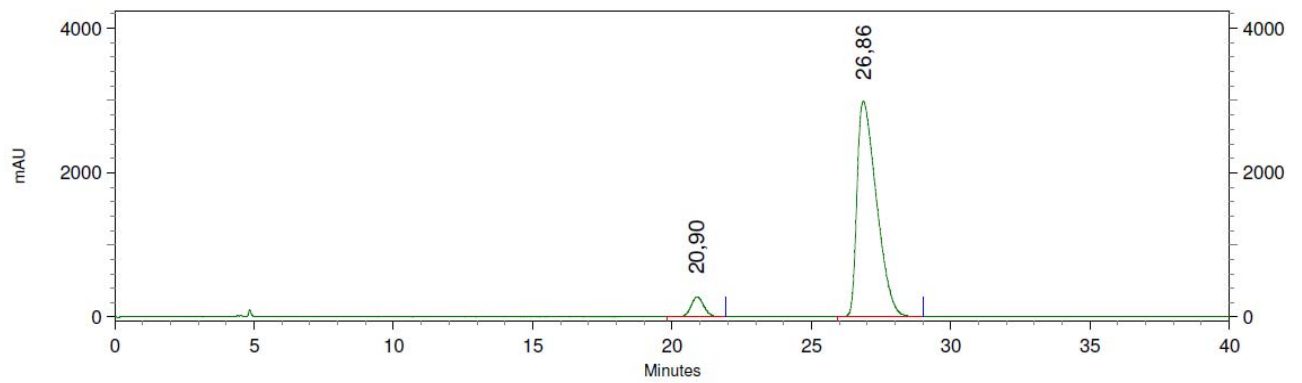

18: $239 \mathrm{~nm}, 4 \mathrm{~nm}$

Results

Retention Time

$\begin{array}{rrr}20,90 & 35171329 & 5,741 \\ 26,86 & 577491925 & 94,259\end{array}$

cis-3ga

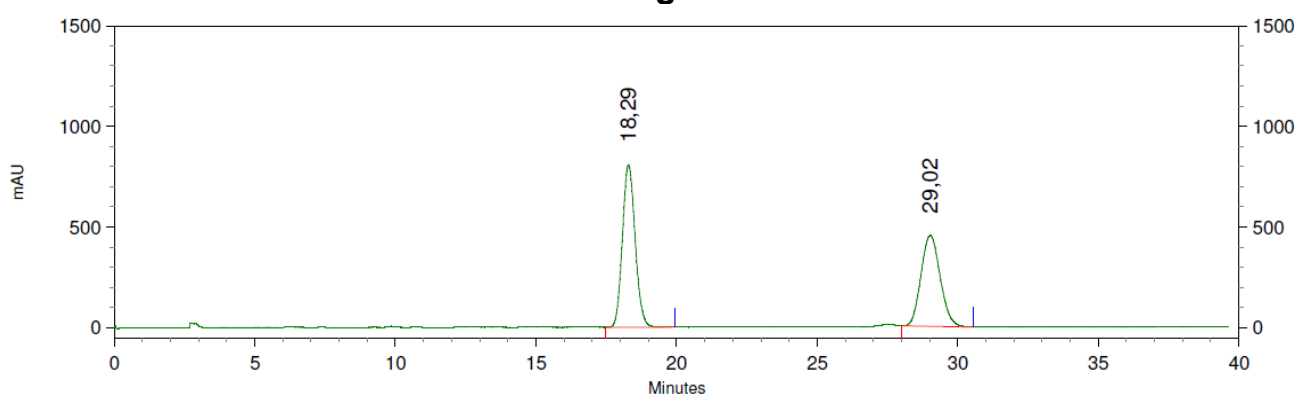

2: $239 \mathrm{~nm}, 4 \mathrm{~nm}$ Results Retention Time

\begin{tabular}{rrr} 
Time & Area & Area \\
\hline 18,29 & 102118473 & 54,447 \\
29,02 & 85437534 & 45,553
\end{tabular}


<smiles>CCCCOC(=O)C1NC(=O)N(c2ccccc2)[C@H]1c1cccc([N+](=O)[O-])c1</smiles>

trans-(4R,5S)-3ha

${ }^{1} \mathrm{H} \mathrm{NMR}, \mathrm{CDCl}_{3}, 300 \mathrm{MHz}$

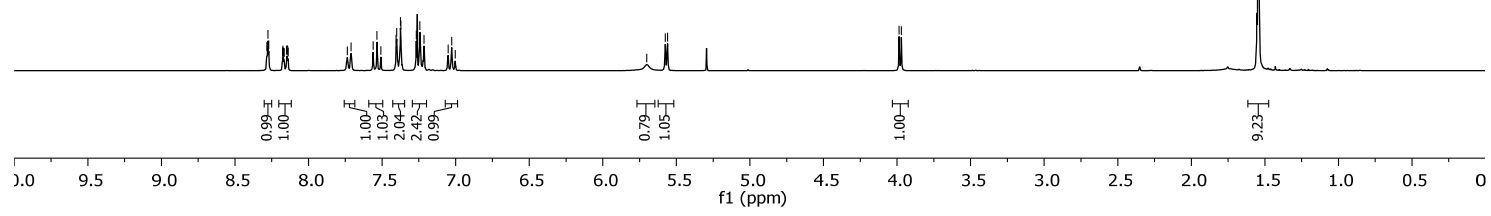<smiles>CCCCOC(=O)C1NC(=O)N(c2ccccc2)[C@H]1c1cccc([N+](=O)[O-])c1</smiles>

trans-(4R,5S)-3ha

${ }^{13} \mathrm{C} \mathrm{NMR}, \mathrm{CDCl}_{3}, 75 \mathrm{MHz}$

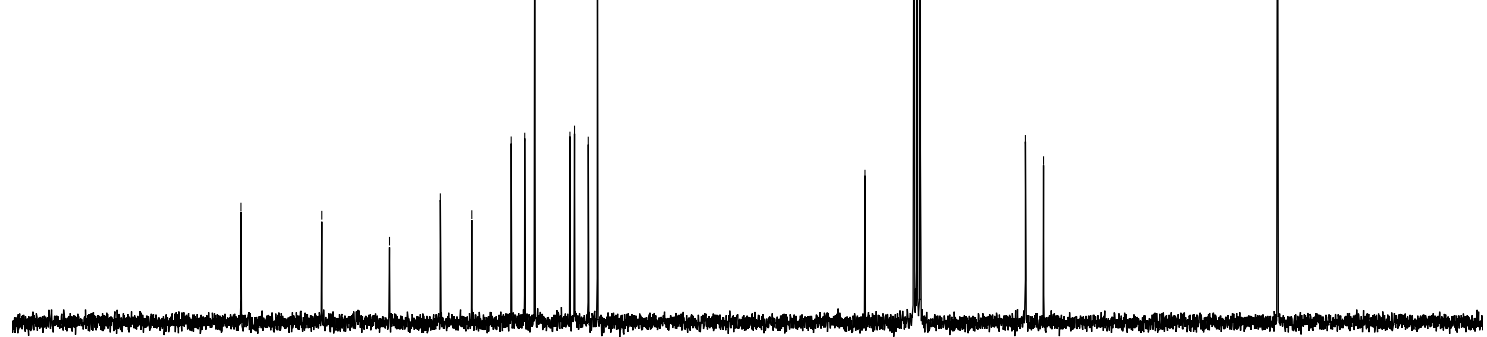
f1 $(100)$ 


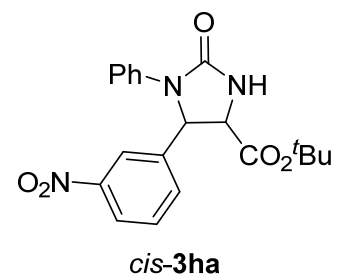

${ }^{1} \mathrm{H} \mathrm{NMR}, \mathrm{CDCl}_{3}, 300 \mathrm{MHz}$

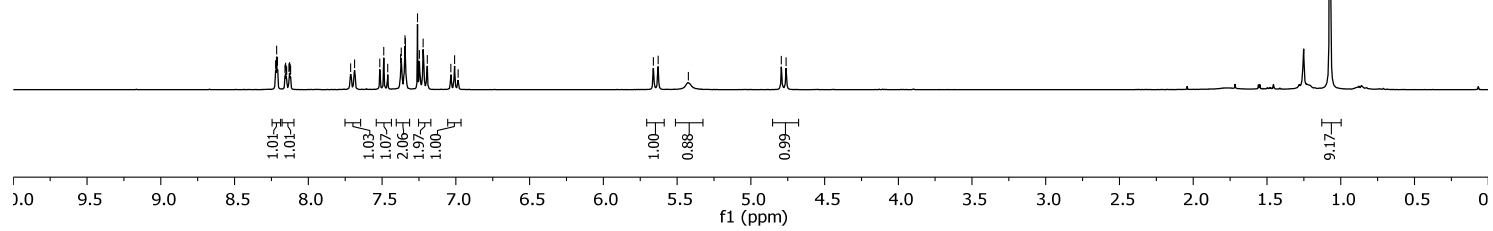

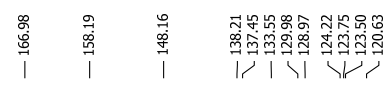

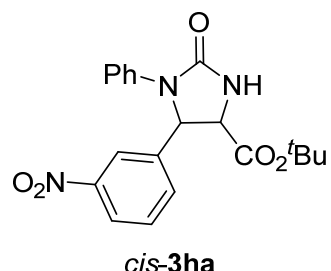

${ }^{13} \mathrm{C} \mathrm{NMR}, \mathrm{CDCl}_{3}, 75 \mathrm{MHz}$

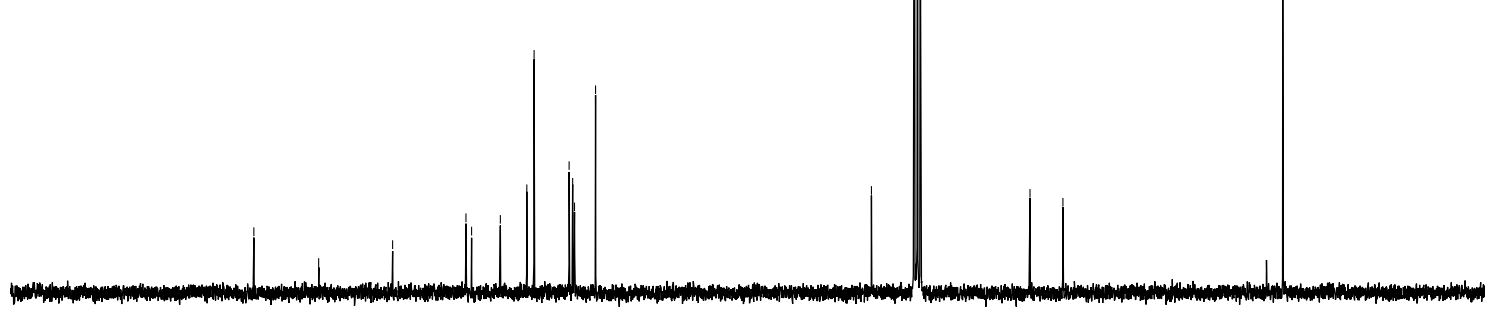

\begin{tabular}{lllllllllllllllllllll}
\hline 0 & 190 & 180 & 170 & 160 & 150 & 140 & 130 & 120 & 110 & $\underset{f 1}{100}(\mathrm{ppm})$ & 90 & 80 & 70 & 60 & 50 & 40 & 30 & 20 & 10
\end{tabular} 


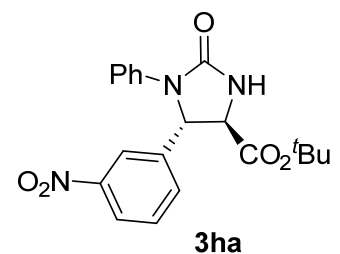

Racemic product (diastereomer mixture)

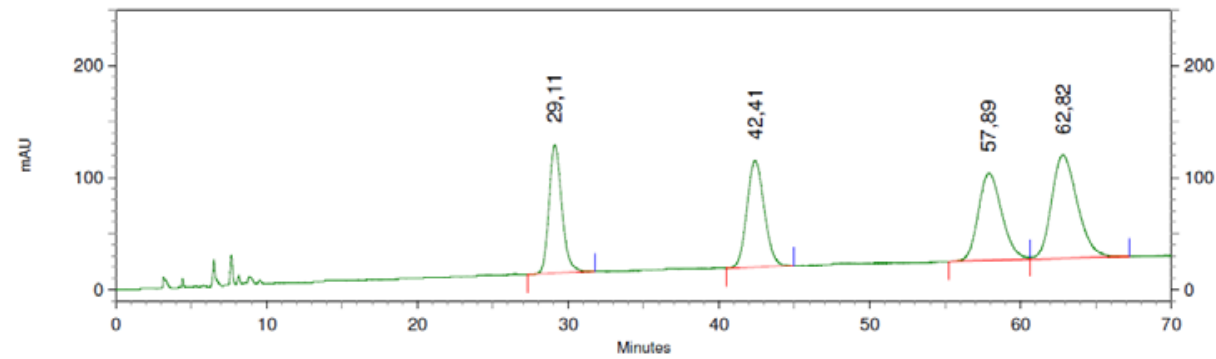

2: $240 \mathrm{~nm}, 4 \mathrm{~nm}$ Results

\begin{tabular}{rrr} 
Retention Time & Area & Area Percent \\
\hline 29,11 & 27150254 & 20,076 \\
42,41 & 30383898 & 22,467 \\
57,89 & 33736216 & 24,946 \\
62,82 & 43966198 & 32,511
\end{tabular}

trans-(4R,5S)-3ha

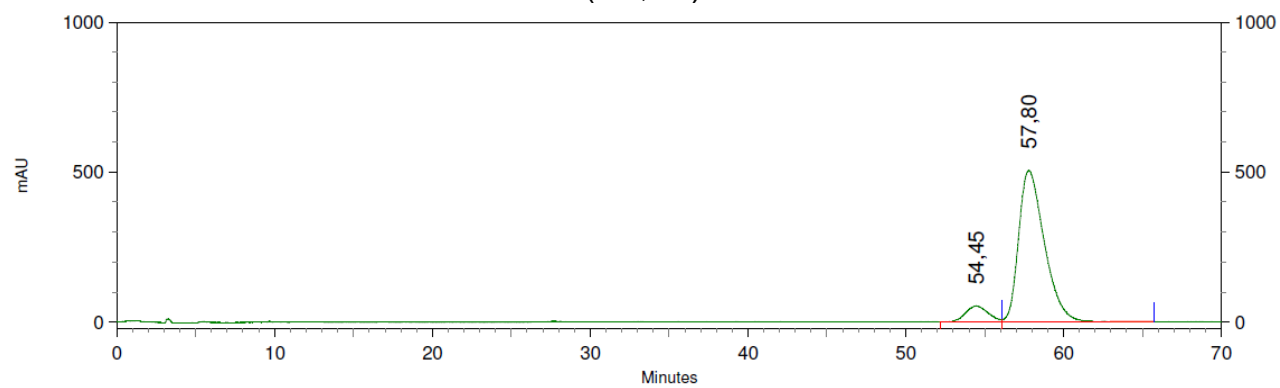

2: $240 \mathrm{~nm}, 4 \mathrm{~nm}$ Results

Retention Time

$\begin{array}{rrr}\text { Time } & \text { Area } & \text { Area } \\ 54,45 & 20845053 & 8,375 \\ 57,80 & 228057477 & 91,625\end{array}$

cis-3ha

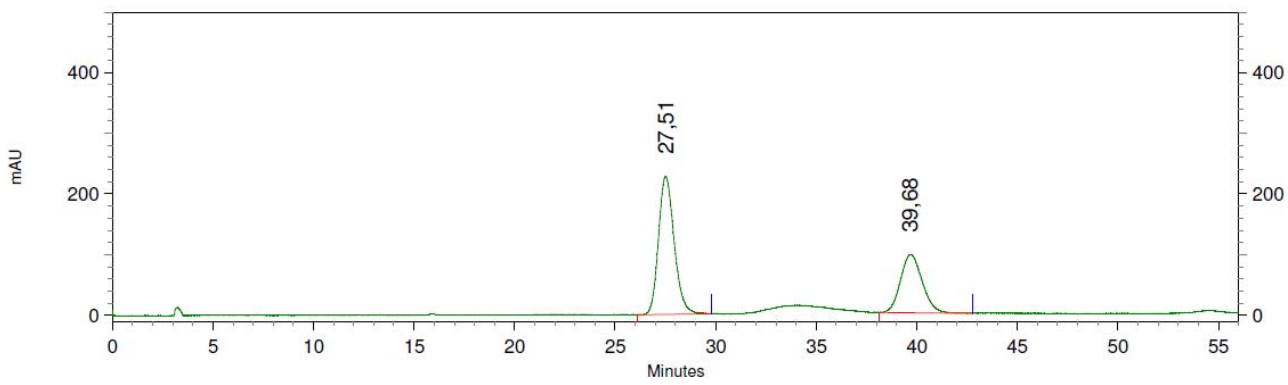

14: $244 \mathrm{~nm}, 4 \mathrm{~nm}$ Results

Retention Time 


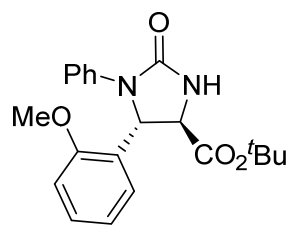

trans-(4R,5S)-3ia

${ }^{1} \mathrm{H} \mathrm{NMR}, \mathrm{CDCl}_{3}, 300 \mathrm{MHz}$

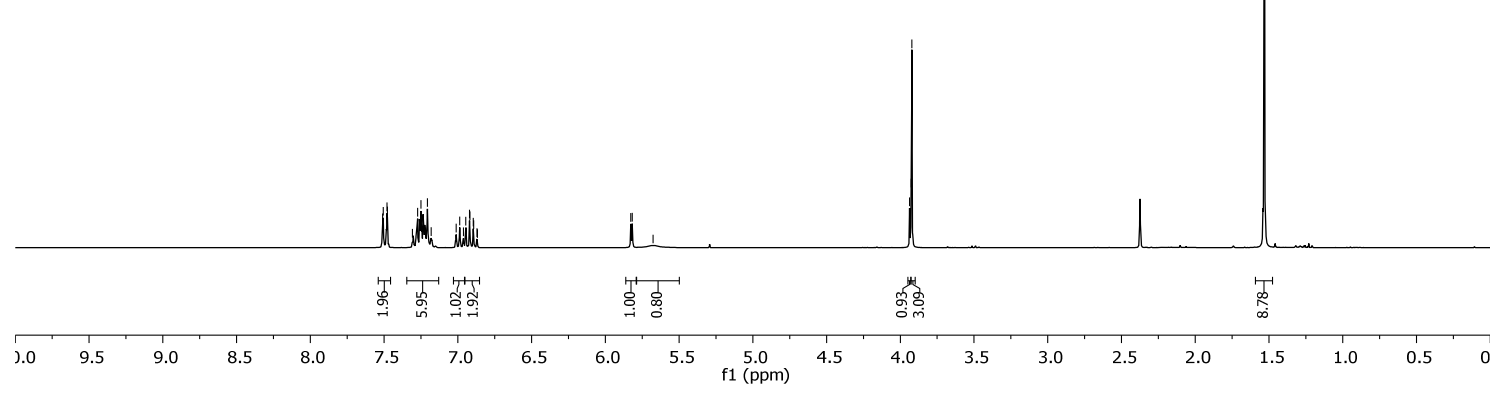

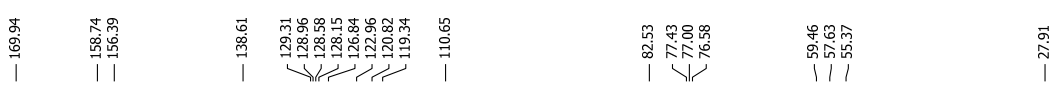<smiles>COC(=O)C1NC(=O)N(c2ccccc2)[C@H]1c1ccccc1OC</smiles>

trans-(4R,5S)-3ia

${ }^{13} \mathrm{C} \mathrm{NMR}, \mathrm{CDCl}_{3}, 75 \mathrm{MHz}$

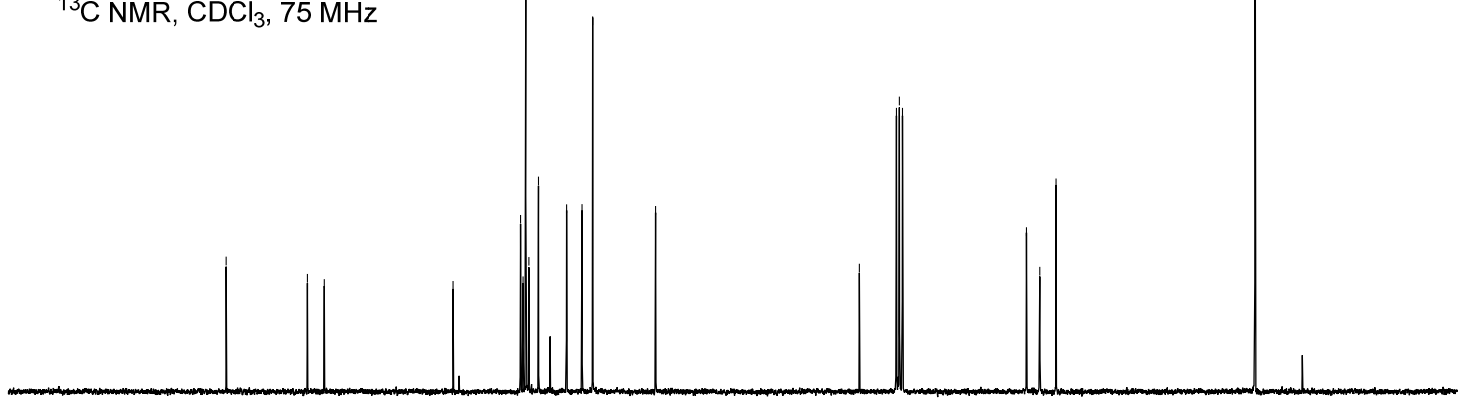

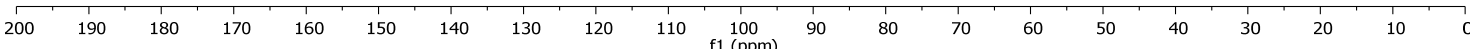




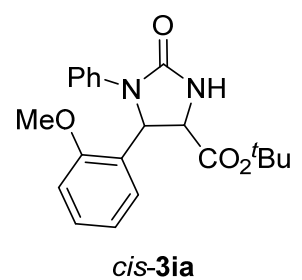

${ }^{1} \mathrm{H} \mathrm{NMR}, \mathrm{CDCl}_{3}, 300 \mathrm{MHz}$
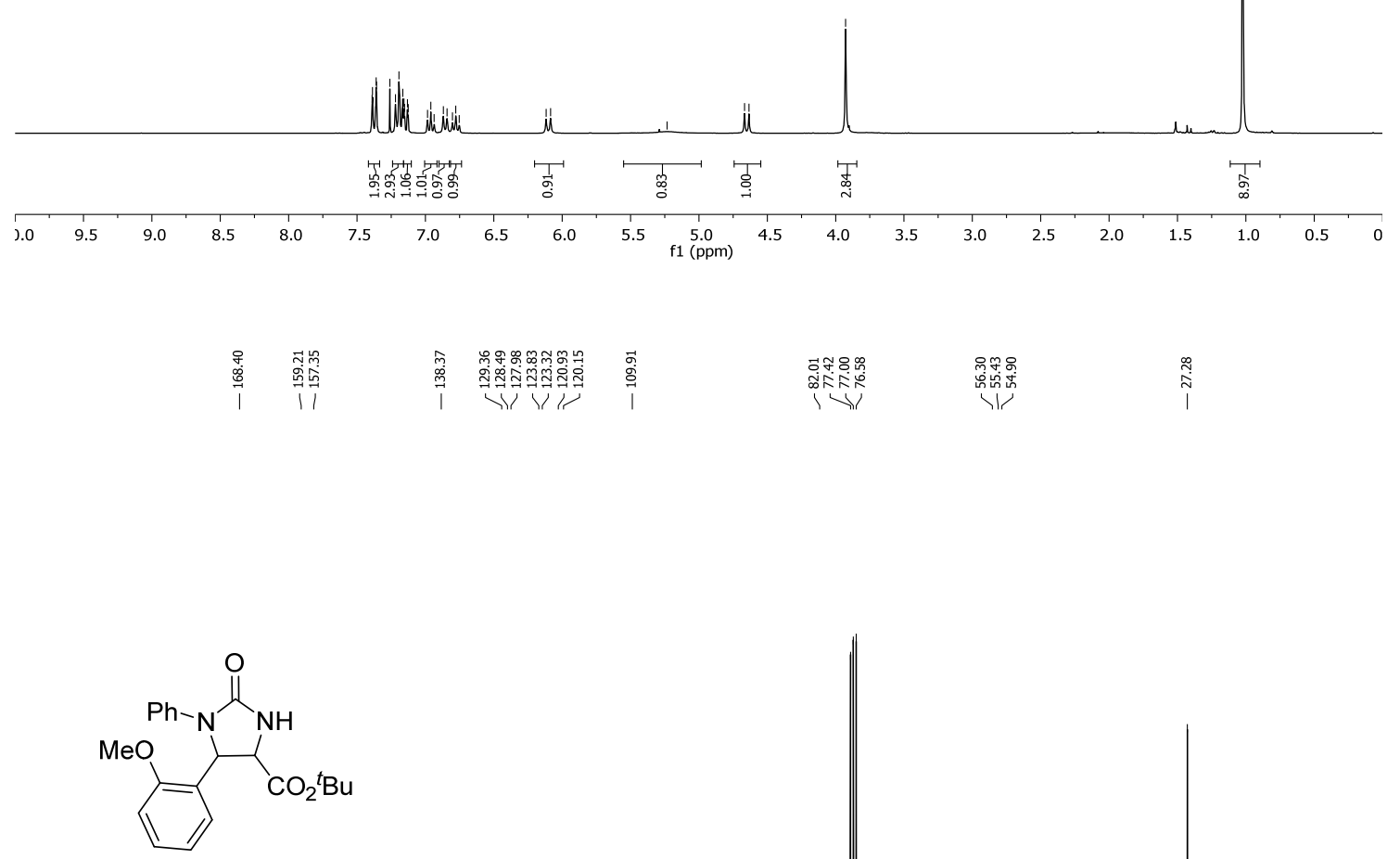

cis-3ia

${ }^{13} \mathrm{C}$ NMR, $\mathrm{CDCl}_{3}, 75 \mathrm{MHz}$

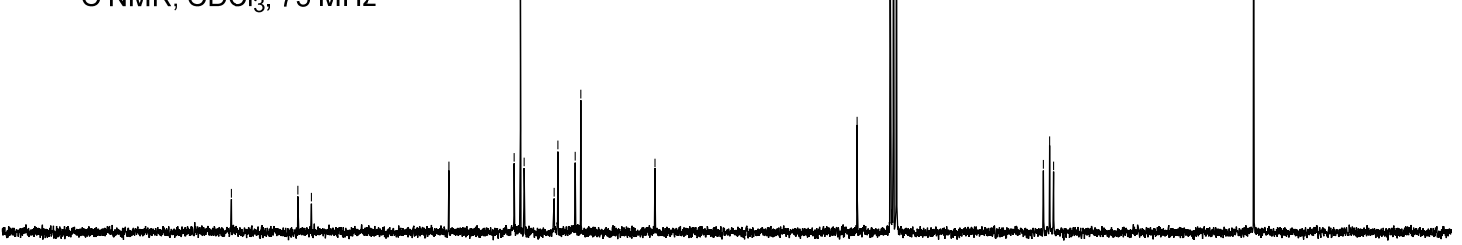

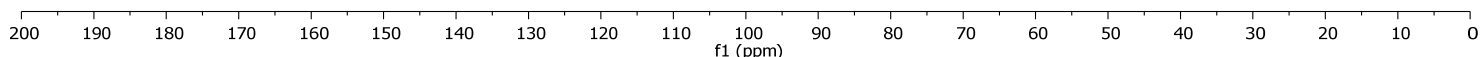




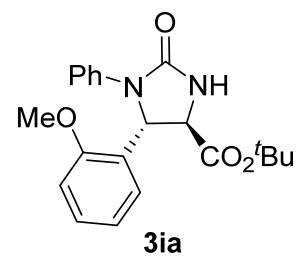

Racemic product (diastereomer mixture)

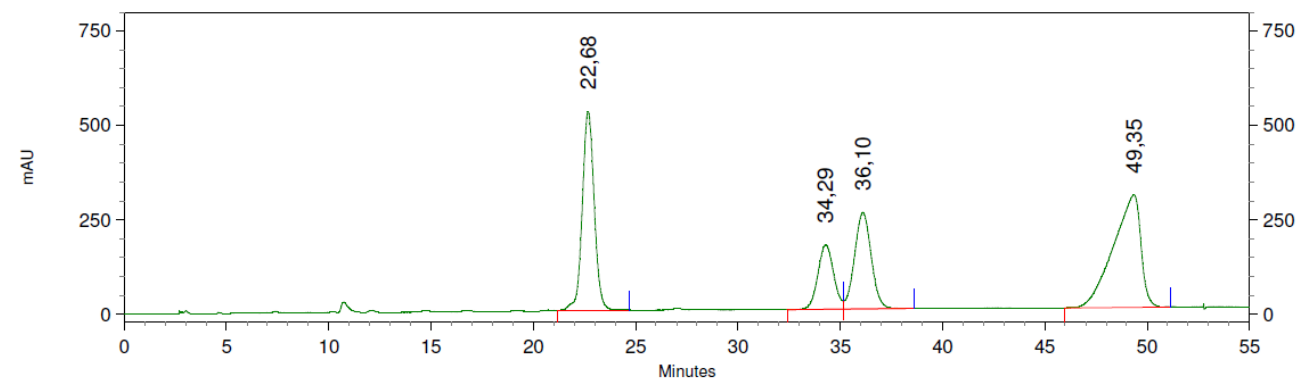

15: $244 \mathrm{~nm}, 4 \mathrm{~nm}$ Results

\begin{tabular}{rrr} 
Retention Time & Area & Area Percent \\
\hline 22,68 & 85554349 & 29,317 \\
34,29 & 36389653 & 12,470 \\
36,10 & 58100336 & 19,909 \\
49,35 & 111784653 & 38,305
\end{tabular}

trans-(4R,5S)-3ia

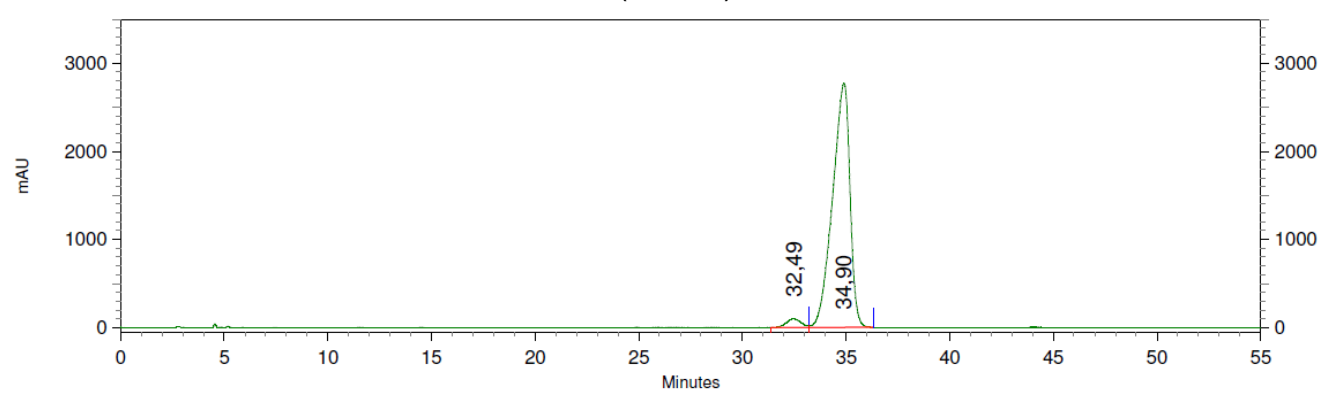

15: $244 \mathrm{~nm}, 4 \mathrm{~nm}$ Results

\begin{tabular}{rrr} 
Retention Time & Area & Area Percent \\
\hline 32,49 & 19295347 & 2,903 \\
34,90 & 645424396 & 97,097
\end{tabular}

cis-3ia

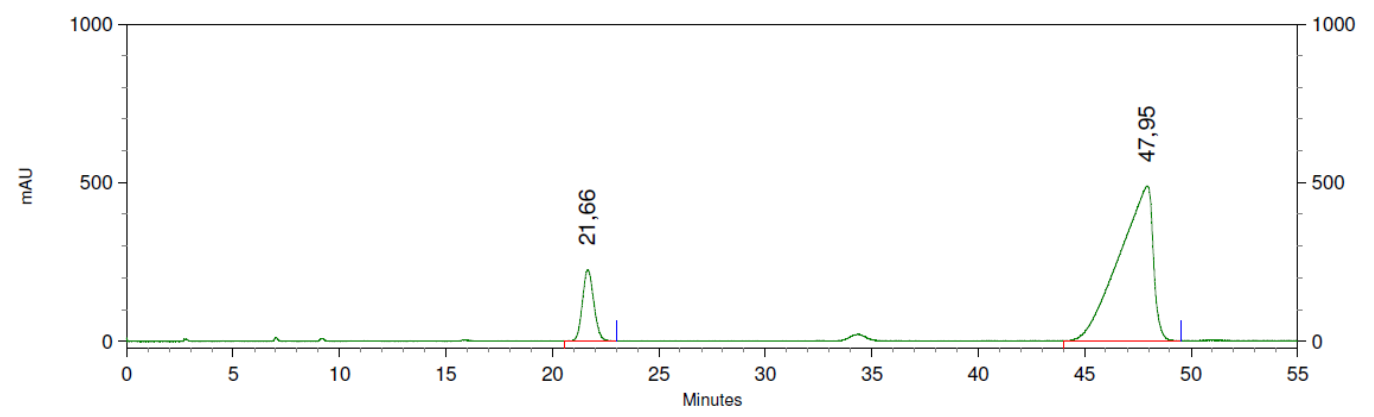

15: $244 \mathrm{~nm}, 4 \mathrm{~nm}$

Results

Retention Time

Area

Area Percent

21,66

33577469

216494976

86,573 


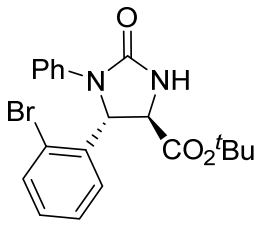

trans-(4R,5S)-3ja

${ }^{1} \mathrm{H} \mathrm{NMR}, \mathrm{CDCl}_{3}, 300 \mathrm{MHz}$

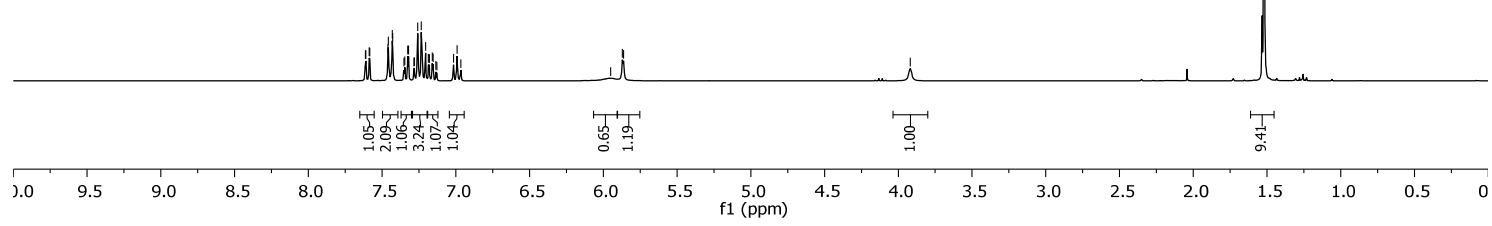

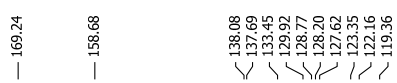

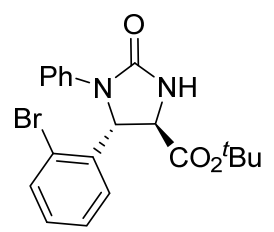

trans-(4R,5S)-3ja

${ }^{13} \mathrm{C} \mathrm{NMR}, \mathrm{CDCl}_{3}, 75 \mathrm{MHz}$

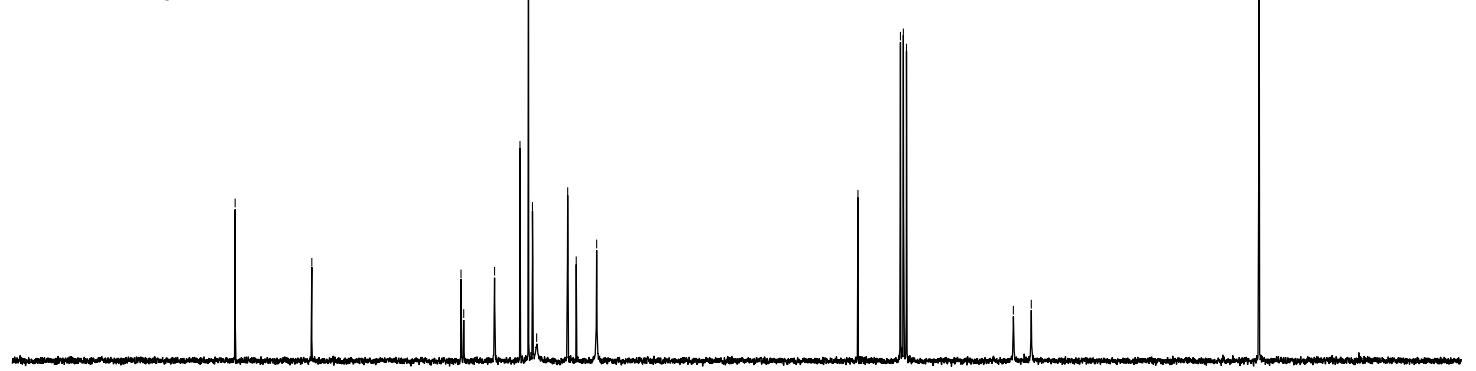

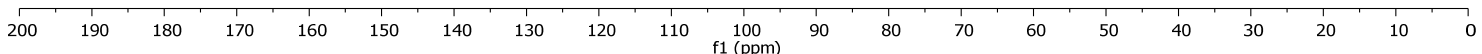




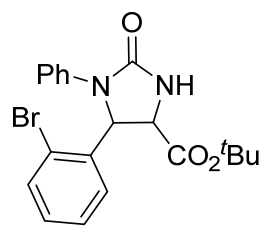

cis-3ja

${ }^{1} \mathrm{H} \mathrm{NMR}, \mathrm{CDCl}_{3}, 300 \mathrm{MHz}$
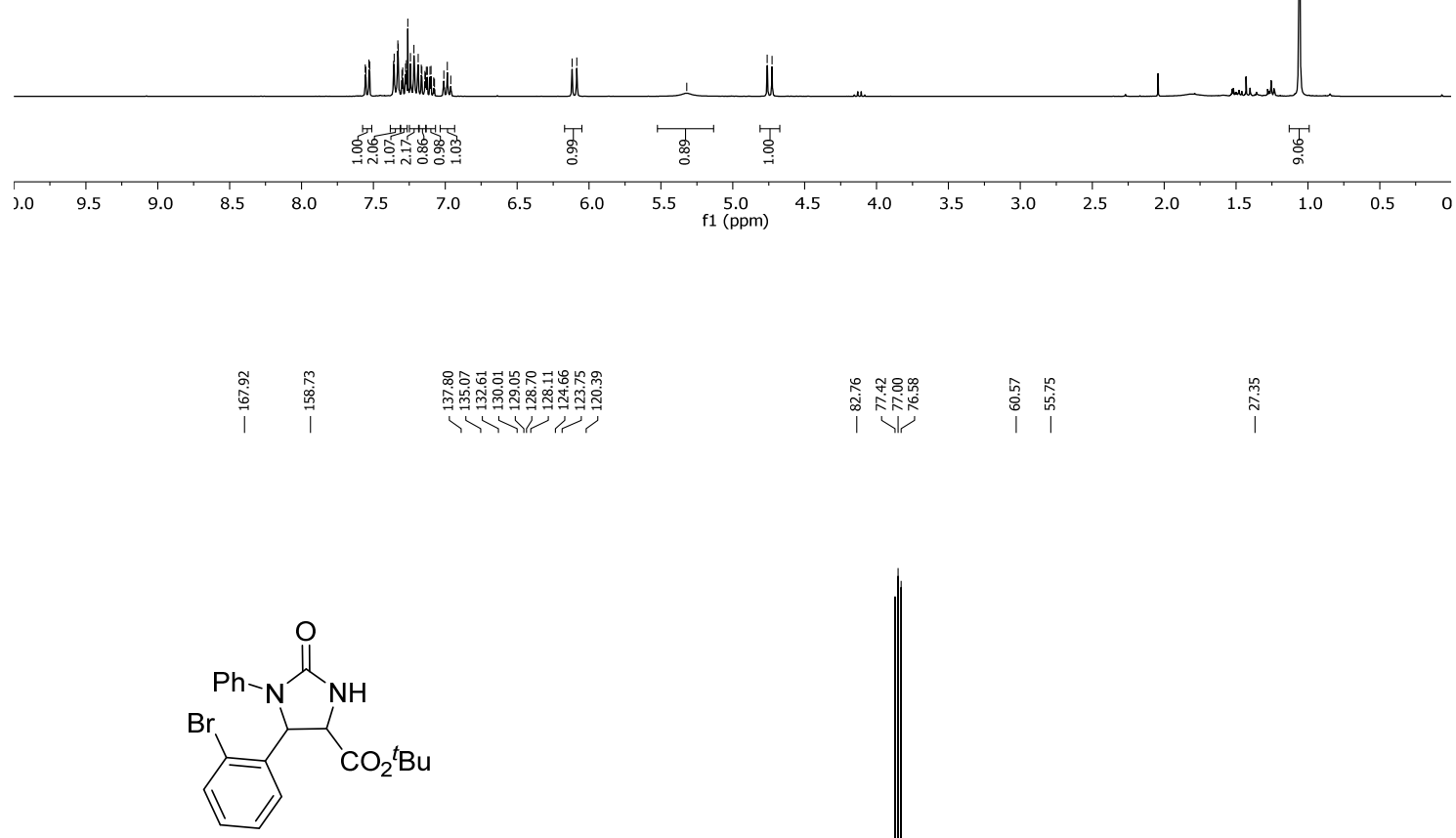

cis-3ja

${ }^{13} \mathrm{C} \mathrm{NMR}, \mathrm{CDCl}_{3}, 75 \mathrm{MHz}$

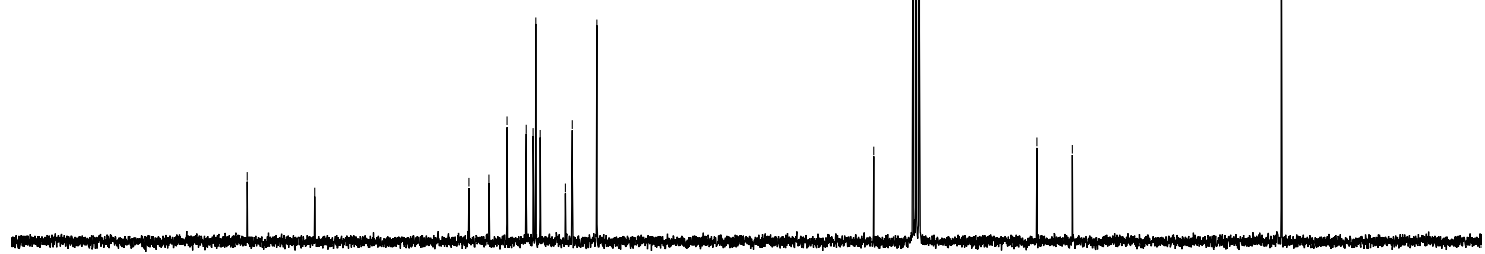

$\begin{array}{lllllllllll}00 & 190 & 180 & 170 & 160 & 150 & 140 & 130 & 120 & 110 & \begin{array}{c}100 \\ \mathrm{f} 1(\mathrm{ppm})\end{array}\end{array}$ 


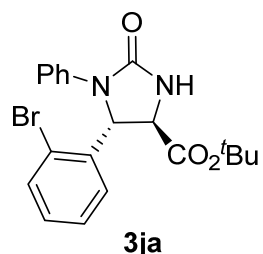

Racemic product (diastereomer mixture)

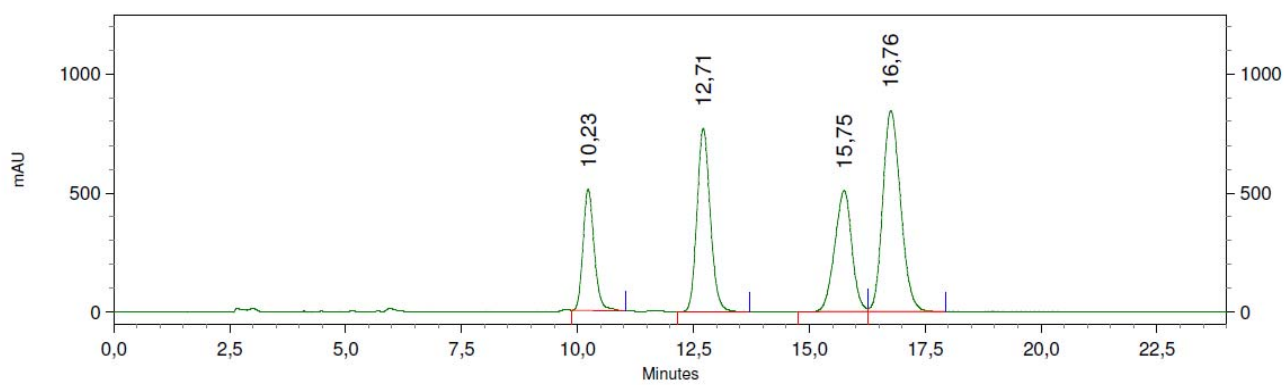

7: $244 \mathrm{~nm}, 4 \mathrm{~nm}$ Results

\begin{tabular}{rrr} 
Retention Time & Area & Area Percent \\
\hline 10,23 & 34514574 & 14,448 \\
12,71 & 61448358 & 25,722 \\
15,75 & 52421894 & 21,944 \\
16,76 & 90508330 & 37,887
\end{tabular}

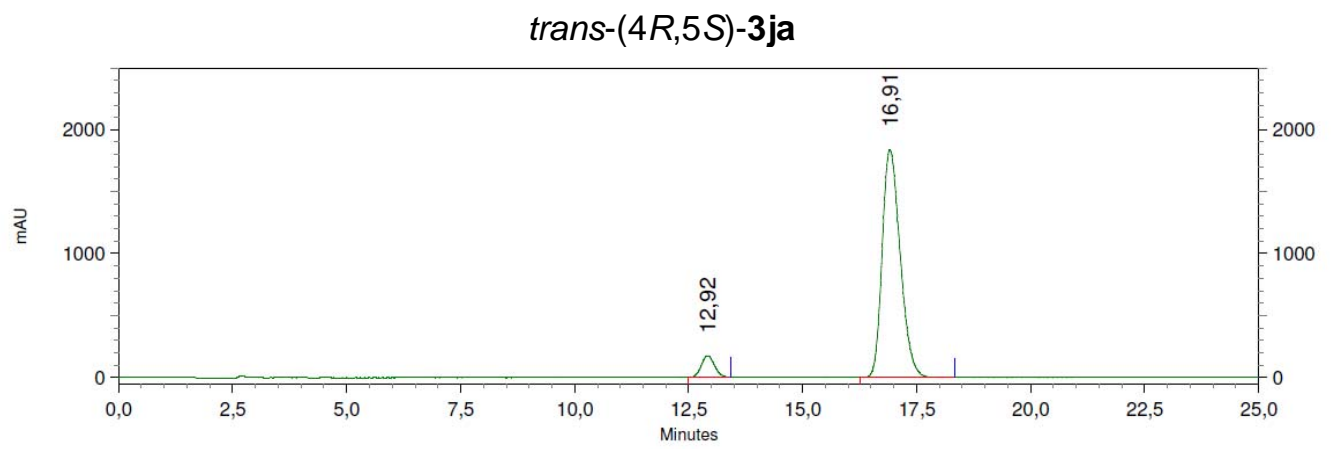

2: $239 \mathrm{~nm}, 4 \mathrm{~nm}$ Results Retention Time

$\begin{array}{rrr}\text { Time } & \text { Area } & \text { Area } \\ 12,92 & 13849605 & 6,409 \\ 16,91 & 202258211 & 93,591\end{array}$

cis-3ja

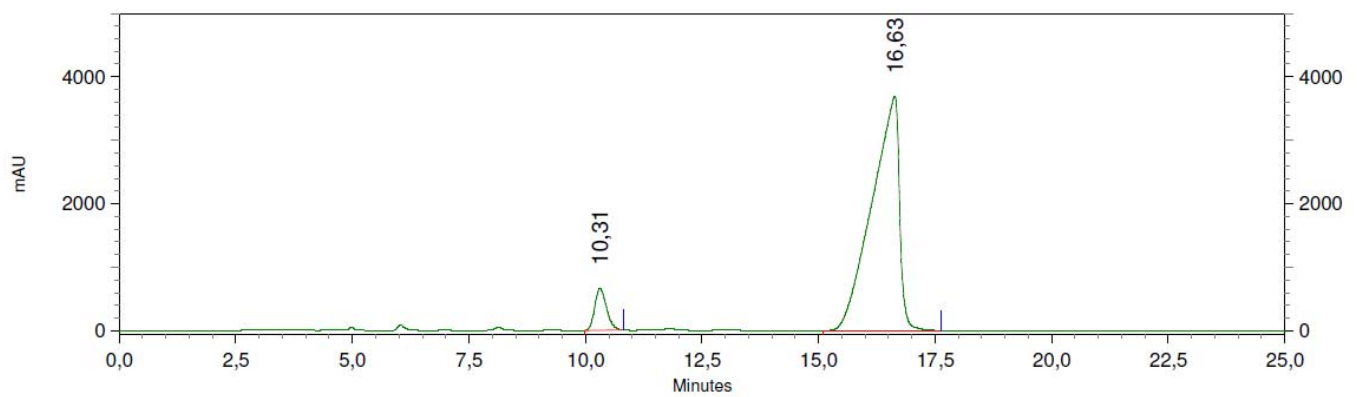

2: $239 \mathrm{~nm}, 4 \mathrm{~nm}$ Results Retention Time 10,31
16,63 Area Area Percent

\begin{tabular}{rrr} 
Retention Time & Area & Area Percent \\
\hline 10,31 & 46050844 & 7,060 \\
16,63 & 606232292 & 92,940
\end{tabular}




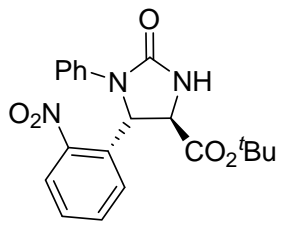

3ka (diastereomer mixture)

${ }^{1} \mathrm{H}$ NMR, $\mathrm{CDCl}_{3}, 300 \mathrm{MHz}$
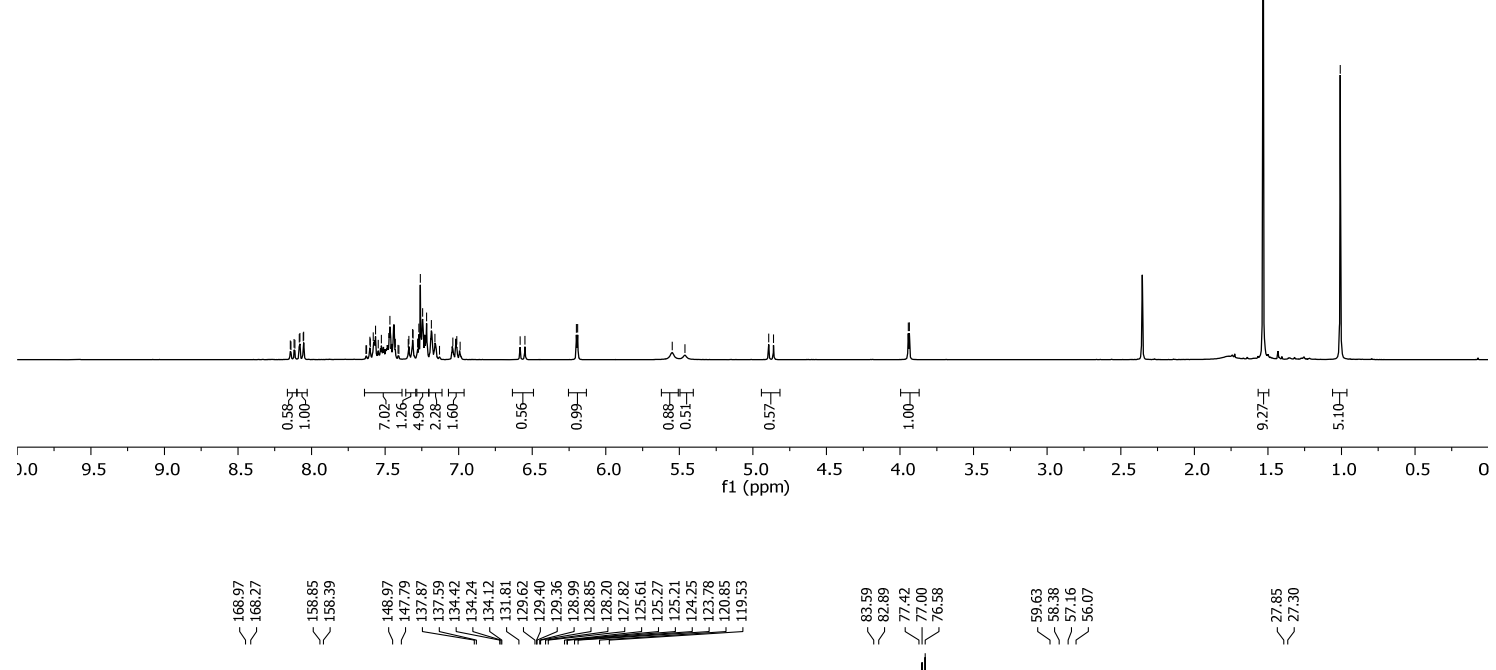

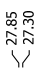

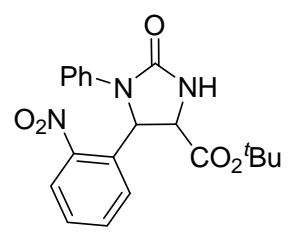

3ka (diastereomer mixture)

${ }^{13} \mathrm{C} \mathrm{NMR}, \mathrm{CDCl}_{3}, 75 \mathrm{MHz}$

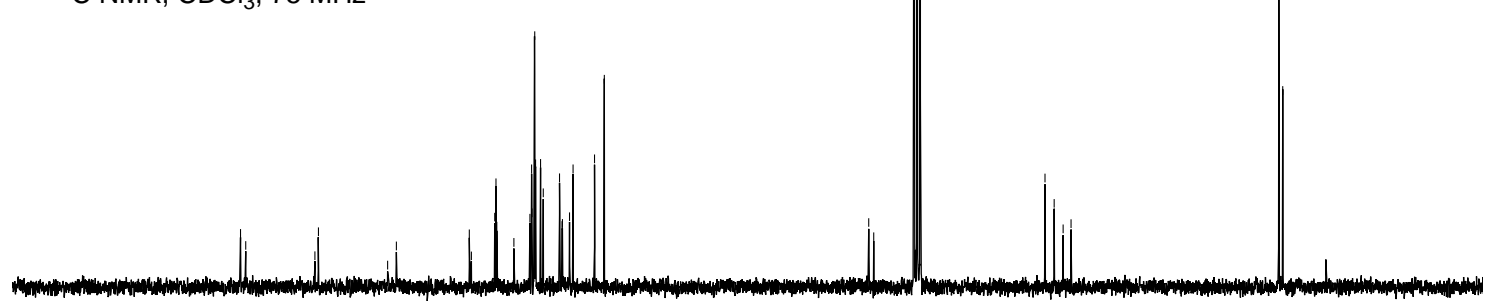

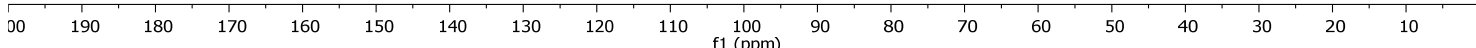




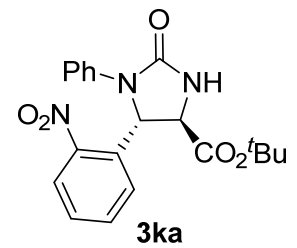

Racemic product (diastereomer mixture)

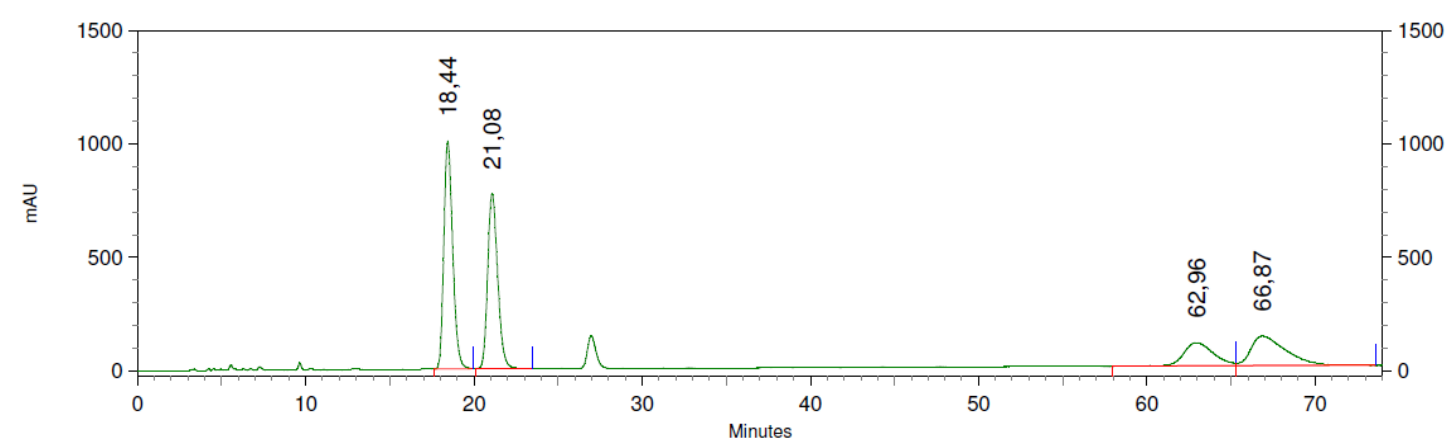

2: 242 nm, 4 nm Results Retention Time

$\begin{array}{lrr}18,44 & 145813850 & 36,548 \\ 21,08 & 127209706 & 31,884 \\ 62,96 & 50854034 & 12,746 \\ 66,87 & 75092783 & 18,822\end{array}$

\section{Enantioselective reaction:}

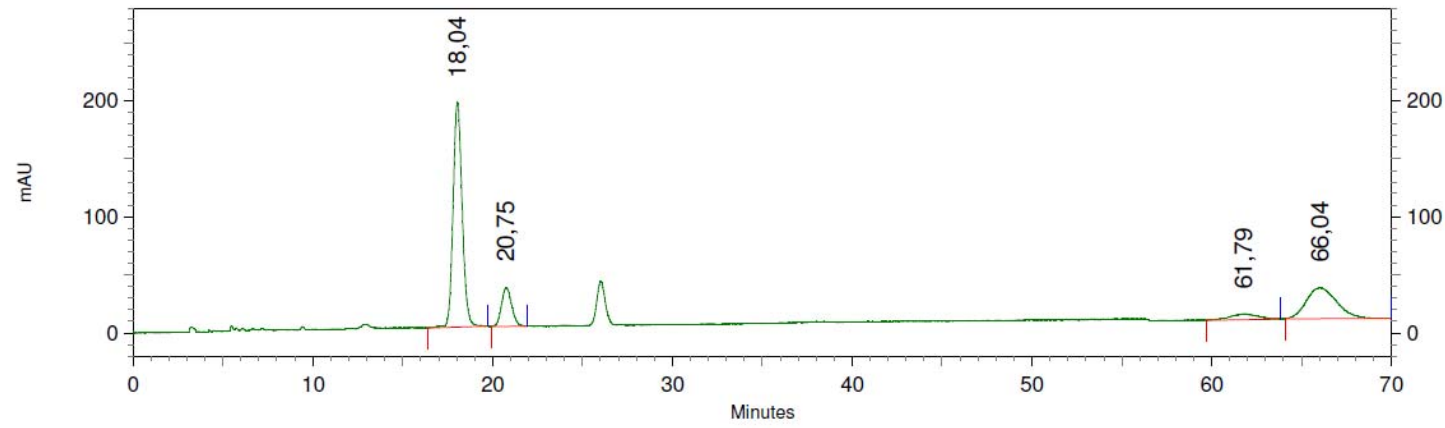

2: 242 nm, 4 nm Results

Retention Time

Area

Area Percent

$\begin{array}{rrr}18,04 & 27157710 & 57,424 \\ 20,75 & 5285579 & 11,176 \\ 61,79 & 2227061 & 4,709 \\ 66,04 & 12622886 & 26,691\end{array}$




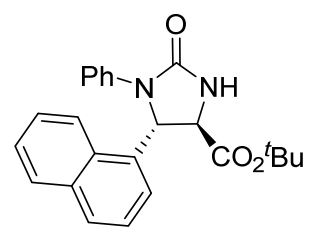

trans-(4R,5S)-3la

${ }^{1} \mathrm{H} \mathrm{NMR}, \mathrm{CDCl}_{3}, 300 \mathrm{MHz}$

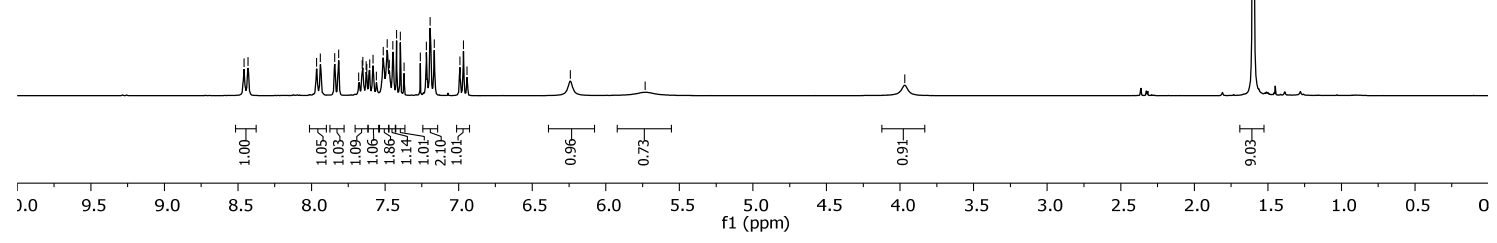

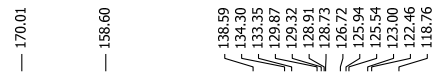

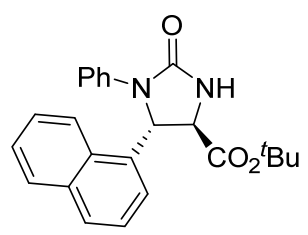

trans-(4R,5S)-3la

${ }^{13} \mathrm{C} \mathrm{NMR}, \mathrm{CDCl}_{3}, 75 \mathrm{MHz}$

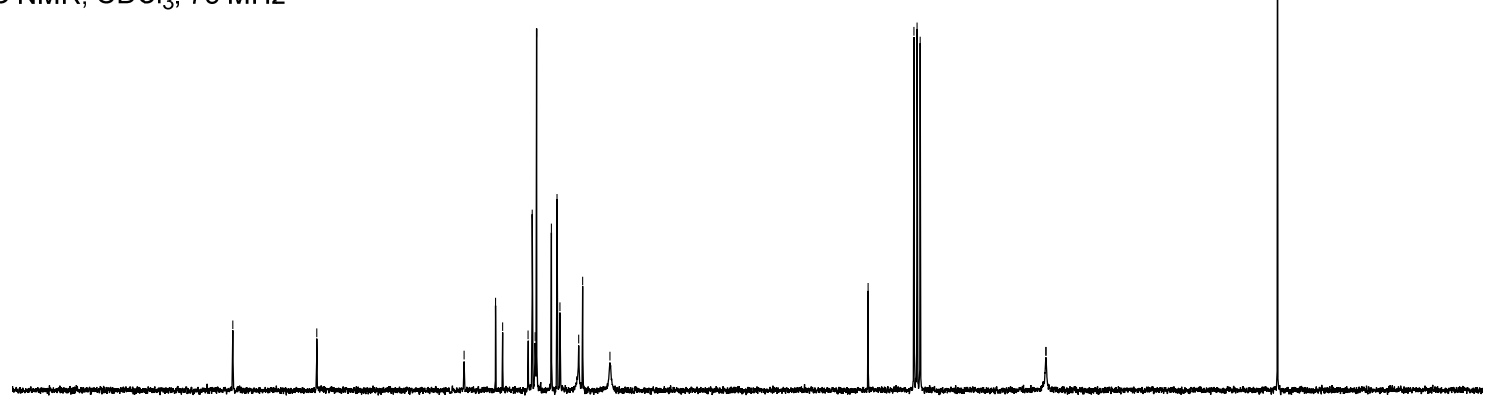

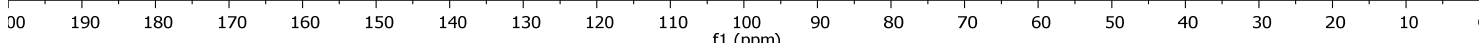




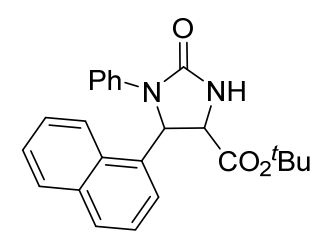

cis-3la

${ }^{1} \mathrm{H} \mathrm{NMR}, \mathrm{CDCl}_{3}, 300 \mathrm{MHz}$

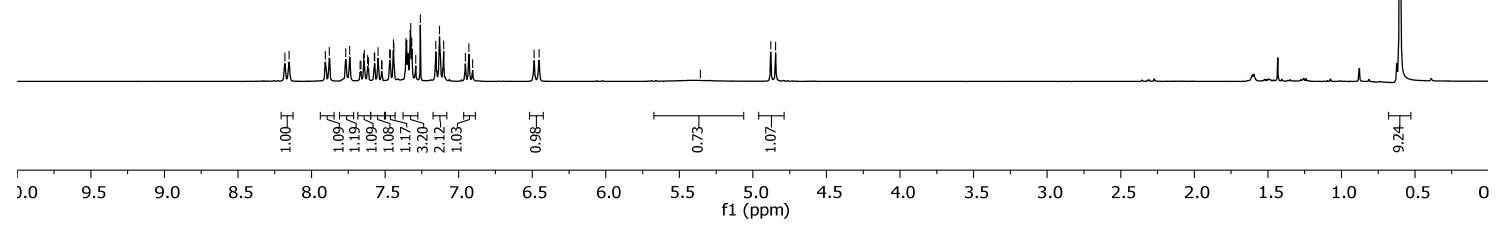

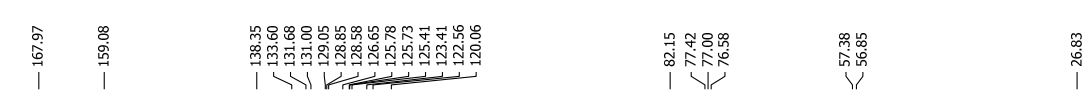

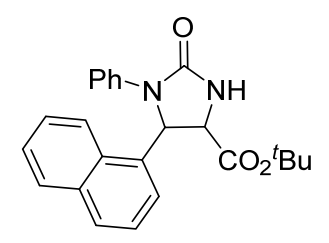

cis-3la

${ }^{13} \mathrm{C} \mathrm{NMR}, \mathrm{CDCl}_{3}, 75 \mathrm{MHz}$

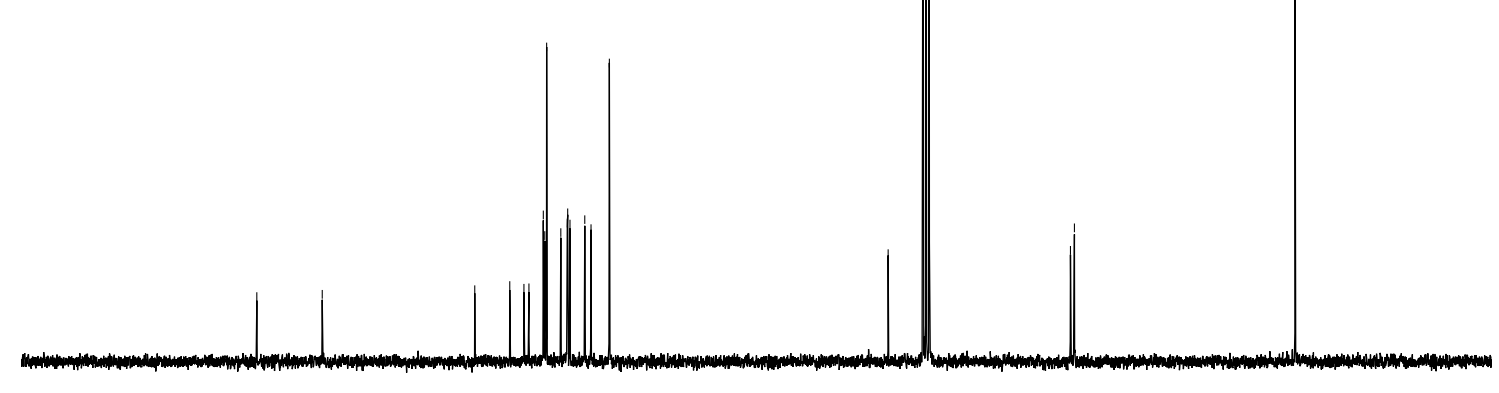

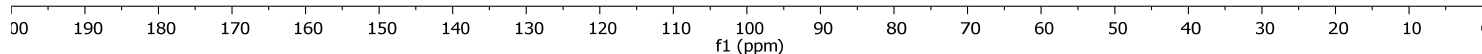




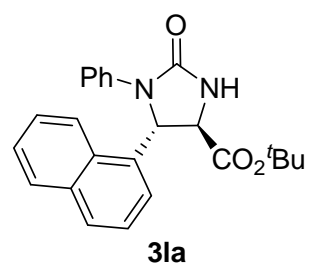

Racemic product (diastereomer mixture)

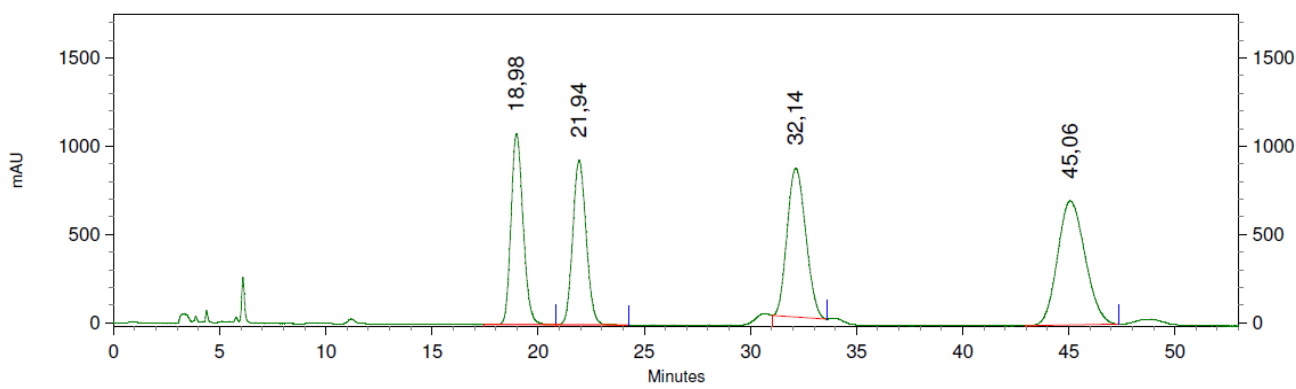

3: $227 \mathrm{~nm}, 4 \mathrm{~nm}$ Results Retention Time

$\begin{array}{rrr}18,98 & \text { Area } & \text { Area } \\ 21,94 & 171010705 & 21,532 \\ 32,14 & 163550050 & 20,593 \\ 45,06 & 205987620 & 25,937 \\ & 253650585 & 31,938\end{array}$

trans-(4R,5S)-3la

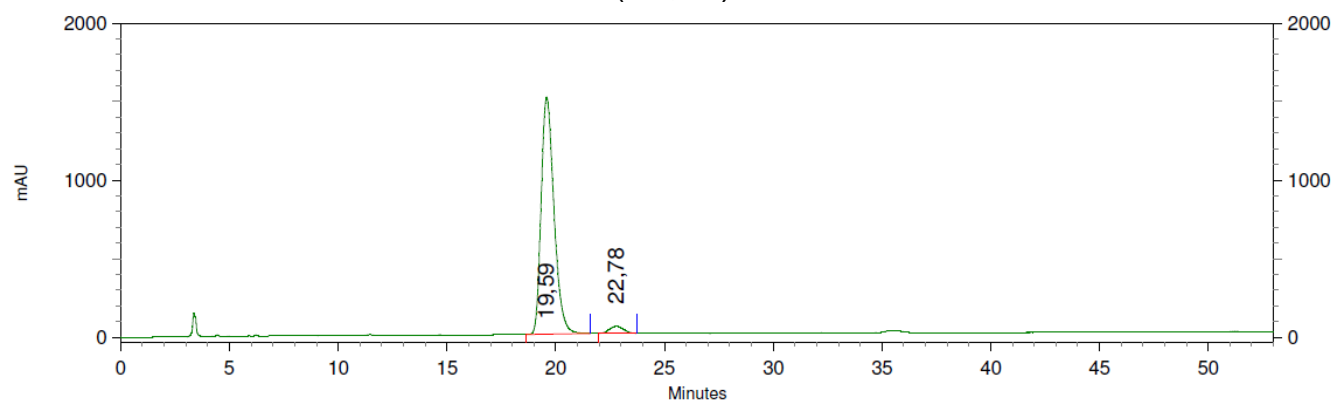

3: $261 \mathrm{~nm}, 4 \mathrm{~nm}$ Results Retention Time

$\begin{array}{rrr}19,59 & \text { Area } & \text { Area } \\ 22,78 & 247452240 & 96,849 \\ & 8050651 & 3,151\end{array}$

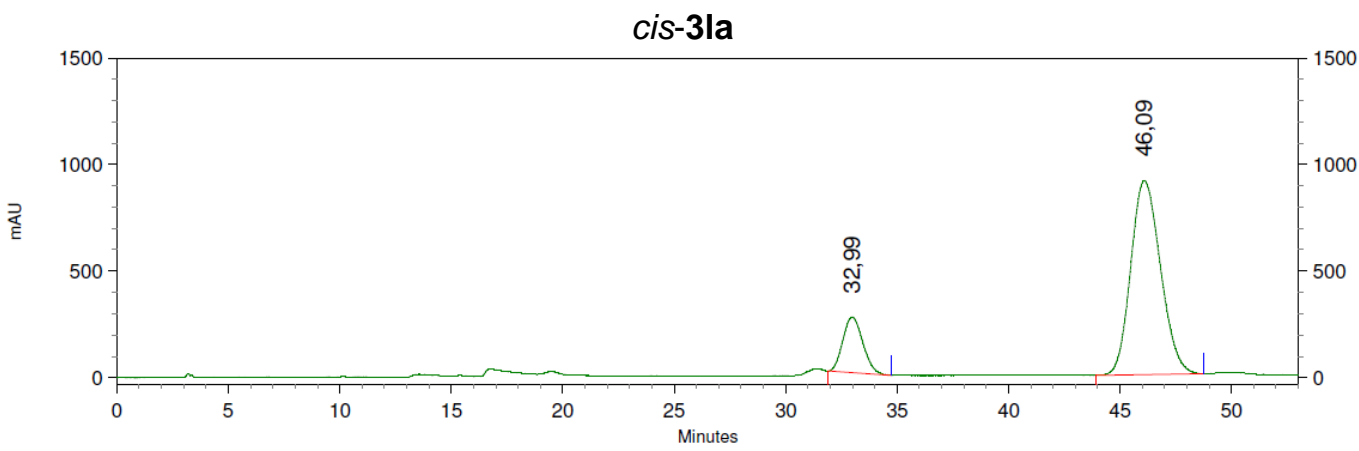

3: $227 \mathrm{~nm}, 4 \mathrm{~nm}$ Results Retention Time

$\begin{array}{rrr}32,99 & 64917046 & 16,124 \\ 46,09 & 337693017 & 83,876\end{array}$




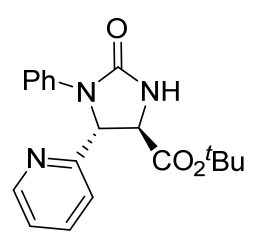

trans-(4R,5S)-3ma

${ }^{1} \mathrm{H} \mathrm{NMR}, \mathrm{CDCl}_{3}, 300 \mathrm{MHz}$

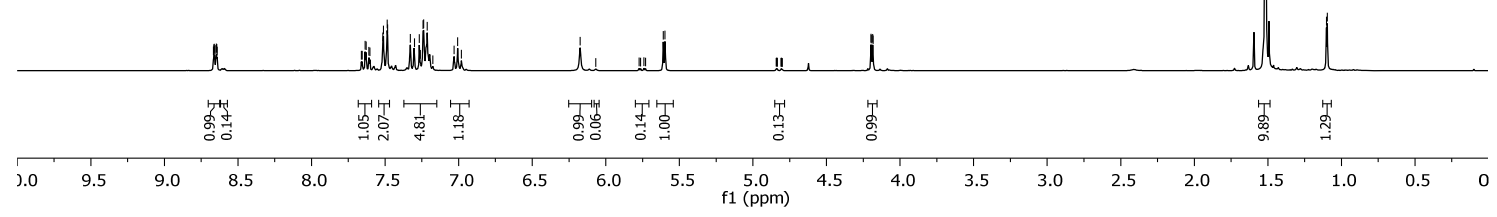

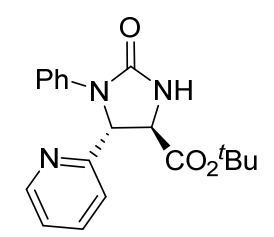

trans-(4R,5S)-3ma

${ }^{13} \mathrm{CNMR} \mathrm{CDCl}_{3}, 75 \mathrm{MHz}$

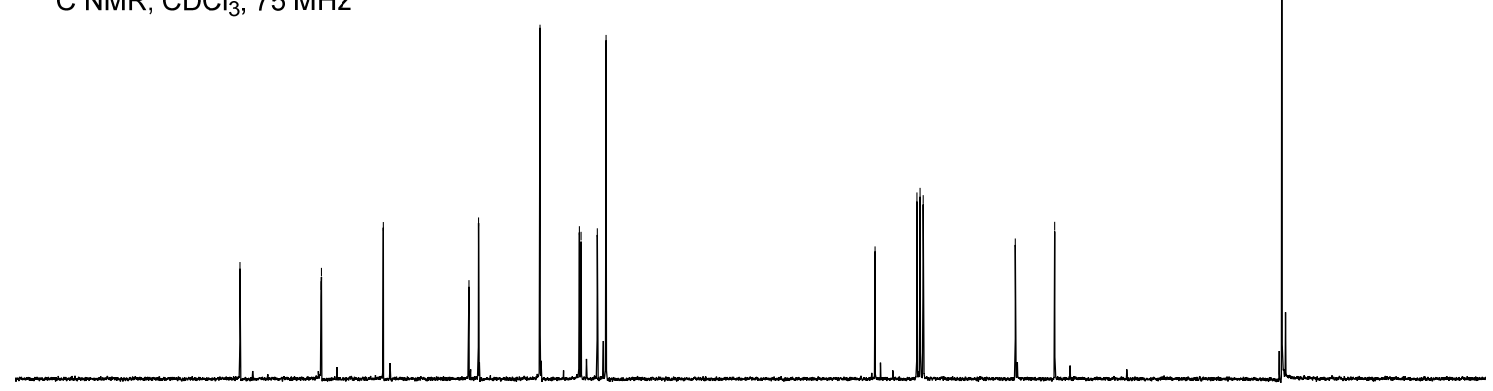

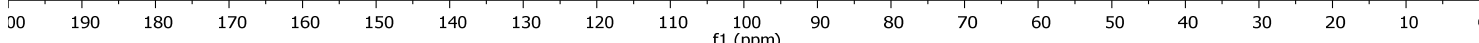




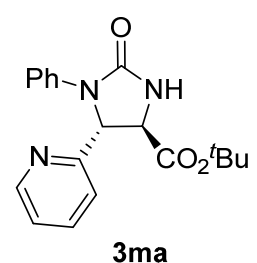

Racemic product (diastereomer mixture)

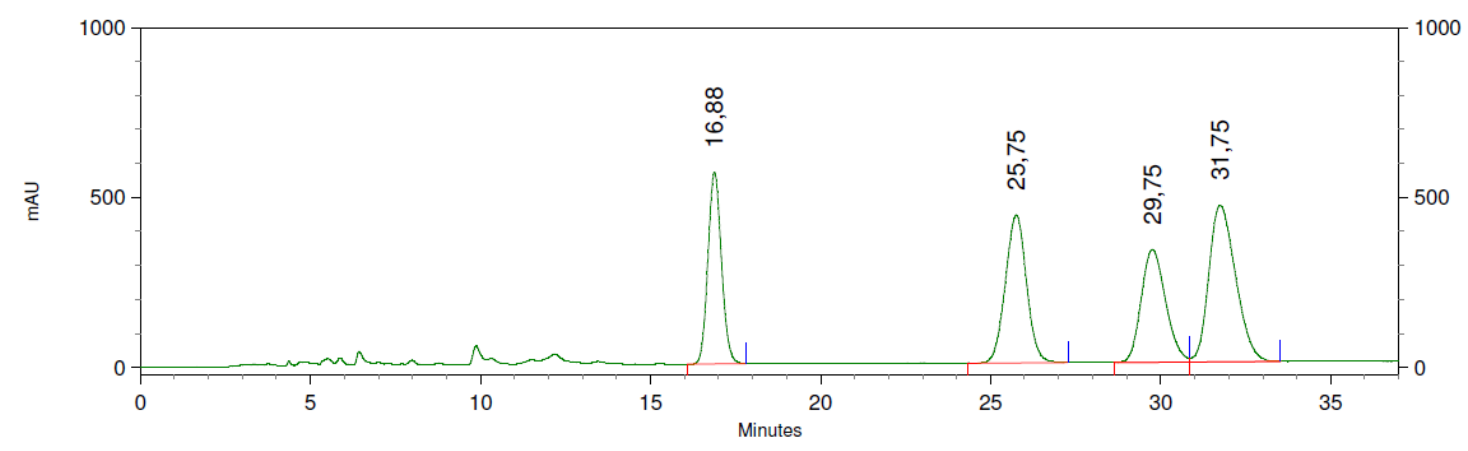

3: $245 \mathrm{~nm}, 4 \mathrm{~nm}$ Results

\begin{tabular}{rrr} 
Retention Time & Area & Area Percent \\
\hline 16,88 & 62469118 & 20,506 \\
25,75 & 75291145 & 24,715 \\
29,75 & 66083824 & 21,693 \\
31,75 & 100791256 & 33,086
\end{tabular}

\section{Enantioselective reaction:}

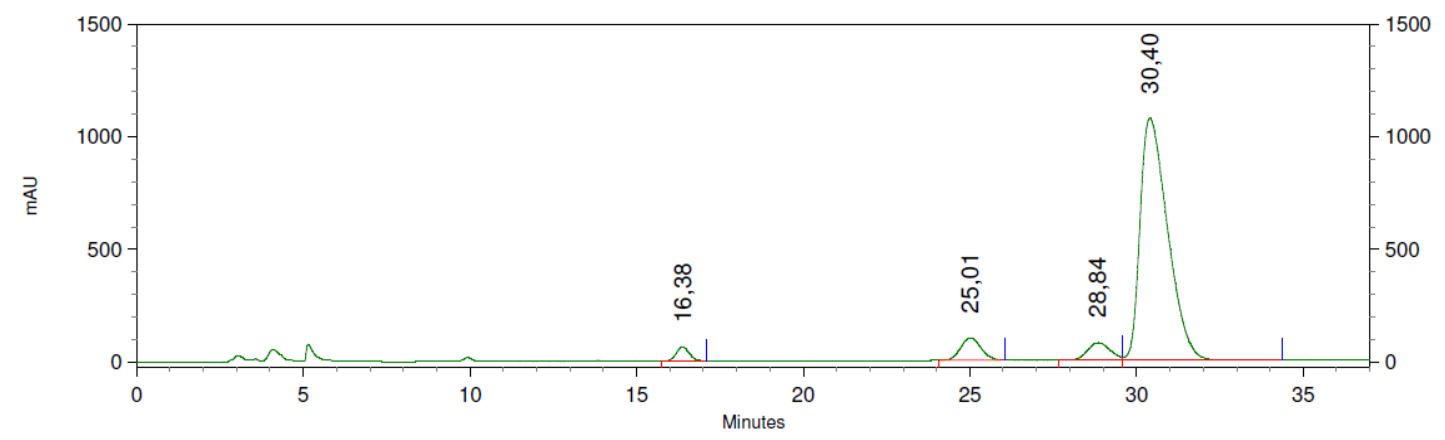

3: $245 \mathrm{~nm}, 4 \mathrm{~nm}$ Results

Retention Time

\begin{tabular}{rrr} 
Time & Area & Area \\
\hline 16,38 & 6823651 & 2,407 \\
25,01 & 16775750 & 5,918 \\
28,84 & 14461615 & 5,102 \\
30,40 & 245404432 & 86,573
\end{tabular}


<smiles>CCOC(=O)C1NC(=O)N(c2ccccc2)C1c1ccccn1</smiles>

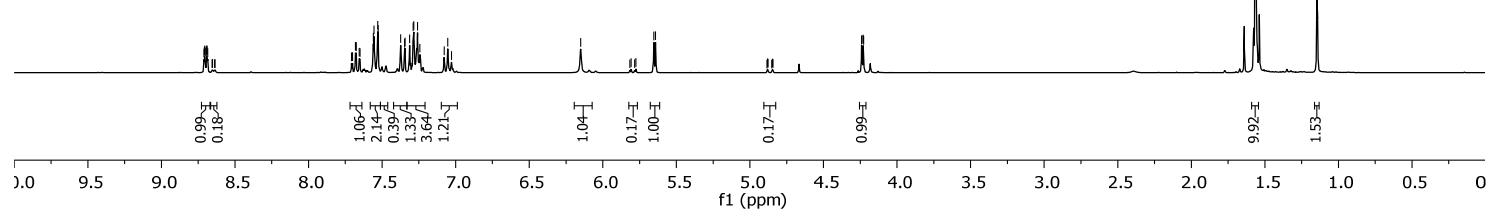<smiles>O=C(OCc1ccccc1)C1NC(=O)N(c2ccccc2)C1c1ccccn1</smiles>

\section{Enantioselective reaction}

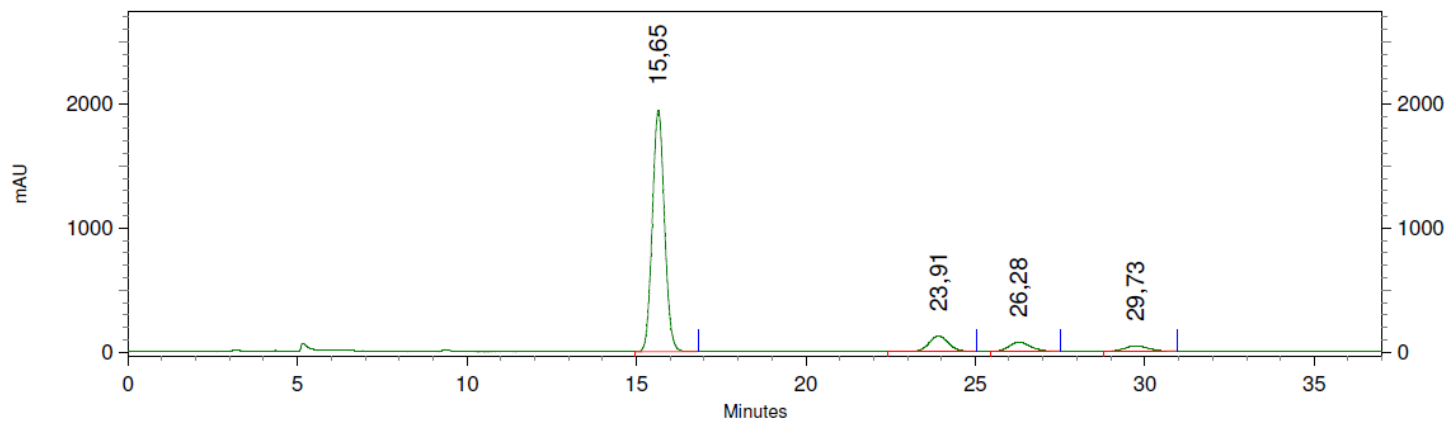

29,73

12644304
8481903

5,391 


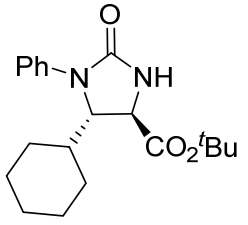

trans-(4R,5S)-3na

${ }^{1} \mathrm{H} \mathrm{NMR}, \mathrm{CDCl}_{3}, 300 \mathrm{MHz}$

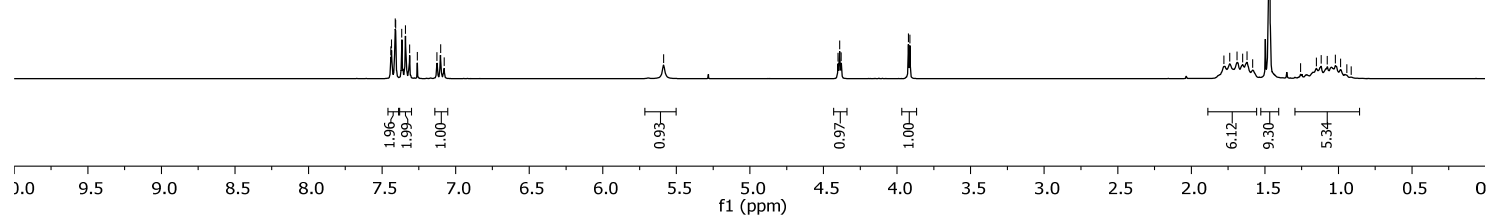

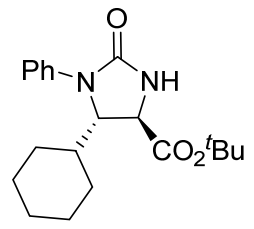

trans-(4R,5S)-3na

${ }^{13} \mathrm{C} \mathrm{NMR}, \mathrm{CDCl}_{3}, 75 \mathrm{MHz}$

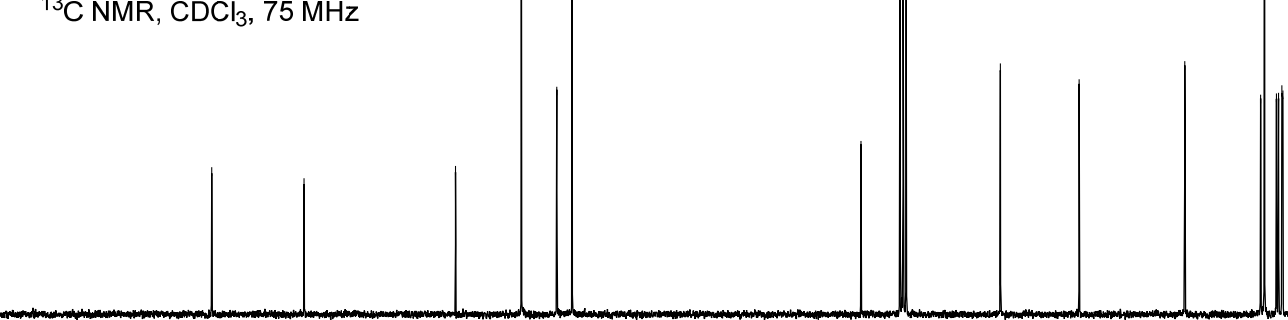

$00 \quad 190$
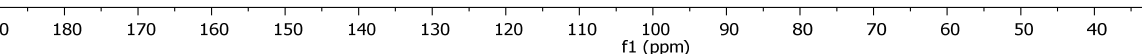


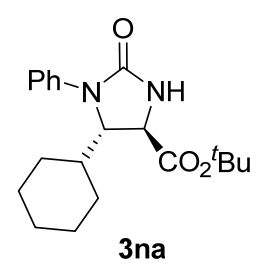

Racemic product (diastereomer mixture)

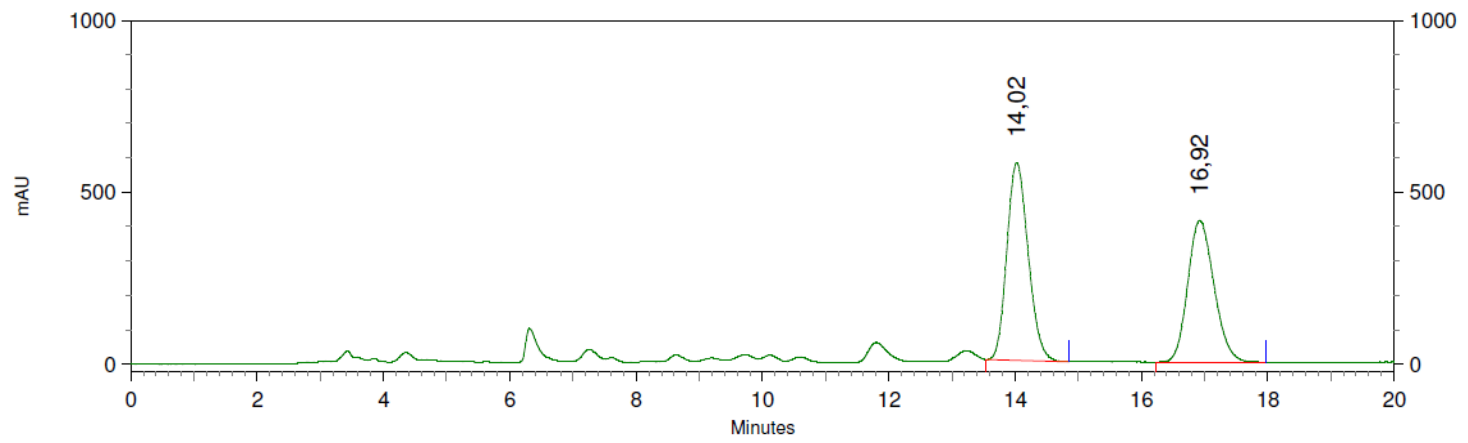

4: $247 \mathrm{~nm}, 4 \mathrm{~nm}$ Results Retention Time

14,02 Area Area Percent

16,92 53843057

53,120

\section{Enantioselective reaction:}

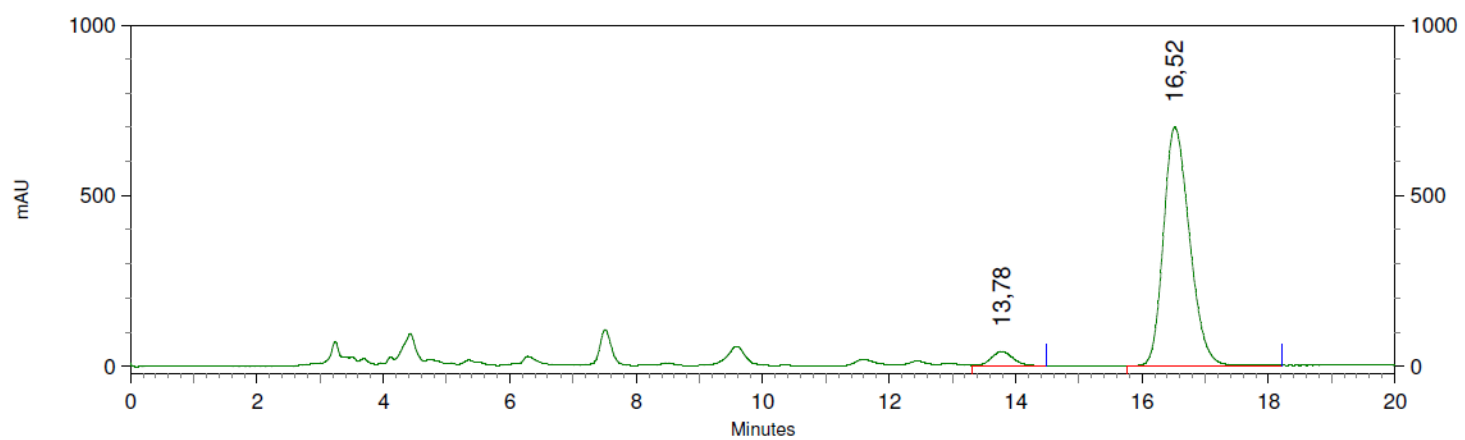

4: 247 nm, 4 nm Results Retention Time

13,78
16,52

Area

Area Percent 


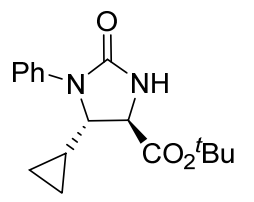

trans-(4R,5S)-3oa

${ }^{1} \mathrm{H} \mathrm{NMR}, \mathrm{CDCl}_{3}, 300 \mathrm{MHz}$
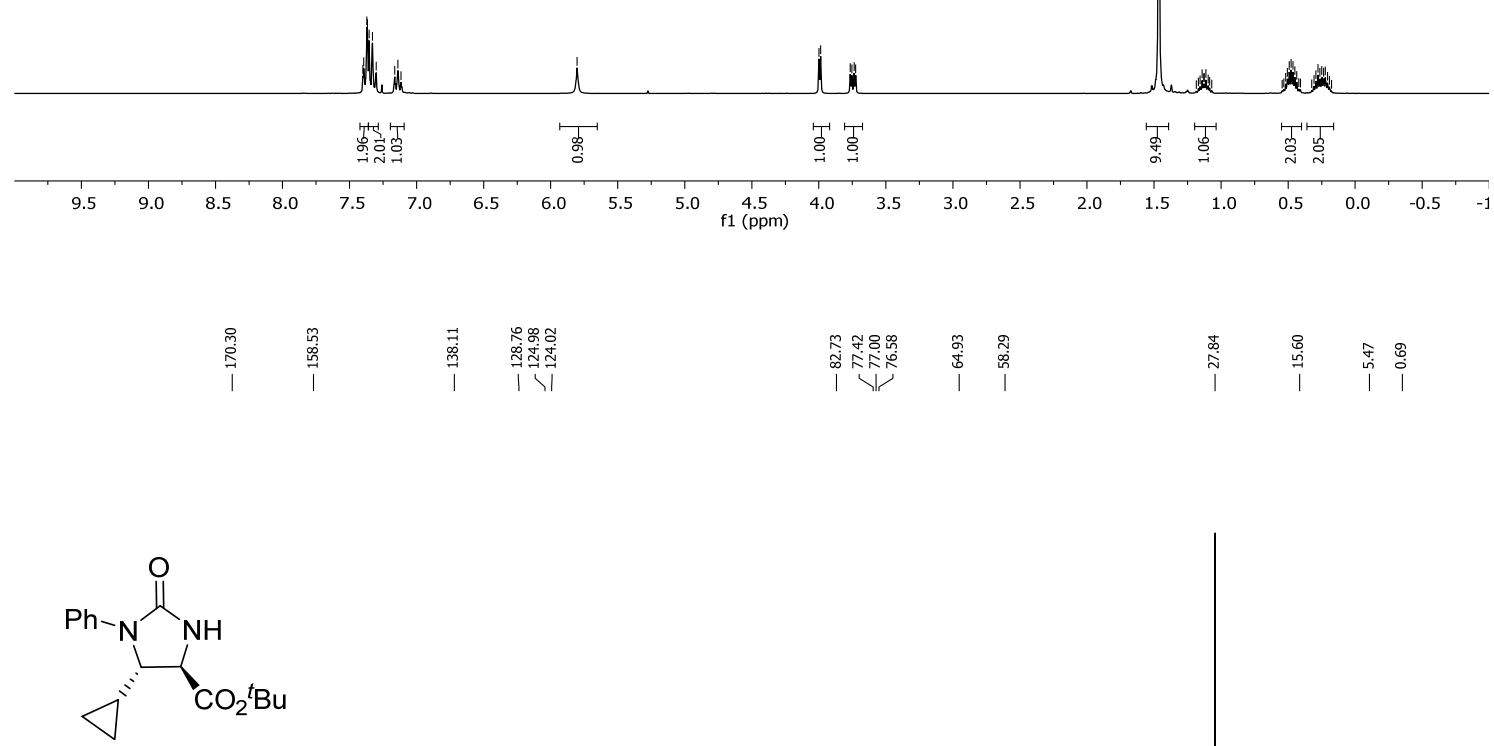

trans-(4R,5S)-3oa ${ }^{13} \mathrm{C} \mathrm{NMR}, \mathrm{CDCl}_{3}, 75 \mathrm{MHz}$

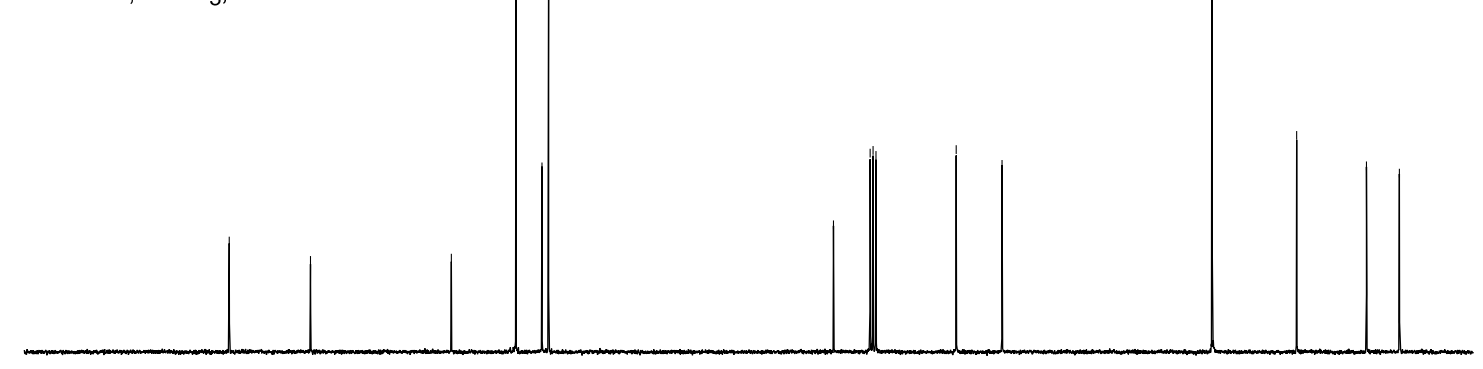

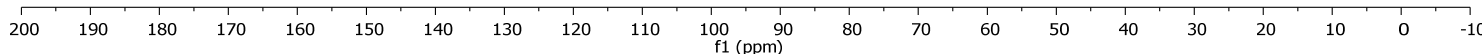


<smiles>CC(C)(C)OC(=O)C1NC(=O)N(c2ccccc2)C1C1CC1</smiles>

cis-3oa

${ }^{1} \mathrm{H} \mathrm{NMR}, \mathrm{CDCl}_{3}, 300 \mathrm{MHz}$

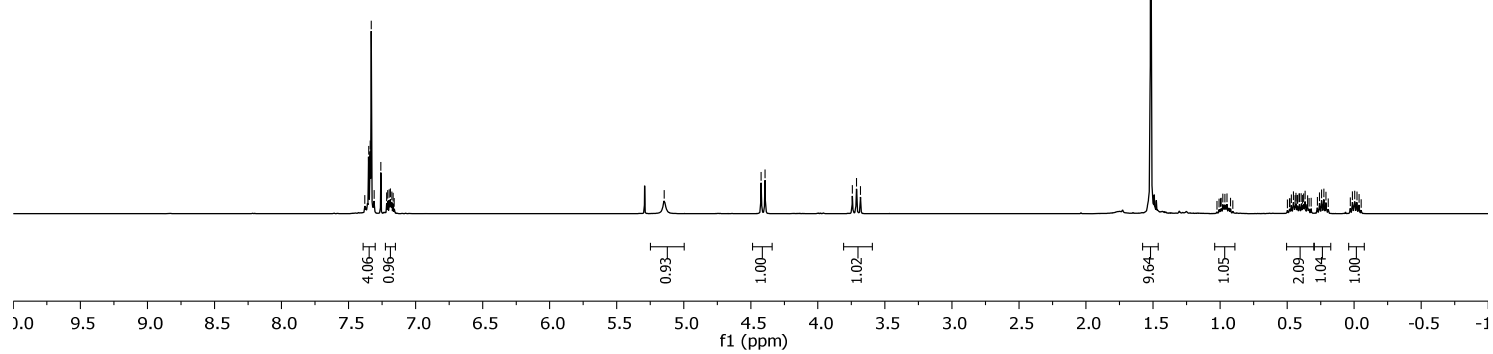

\begin{tabular}{|c|c|c|c|c|c|c|c|}
\hline 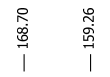 & 寽 & 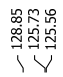 & 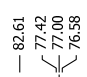 & 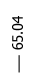 & $\stackrel{\substack{\stackrel{h}{h} \\
\text { hn }}}{\mid}$ & $\begin{array}{l}\text { o } \\
\stackrel{\infty}{\infty} \\
\text { । }\end{array}$ & $\begin{array}{l}\text { D. } \\
\stackrel{0}{0} \\
\overrightarrow{0}\end{array}$ \\
\hline
\end{tabular}<smiles>CCCCC(C)(C)OC(=O)C1NC(=O)N(c2ccccc2)C1C1CC1</smiles>

${ }^{13} \mathrm{C} \mathrm{NMR}, \mathrm{CDCl}_{3}, 75 \mathrm{MHz}$

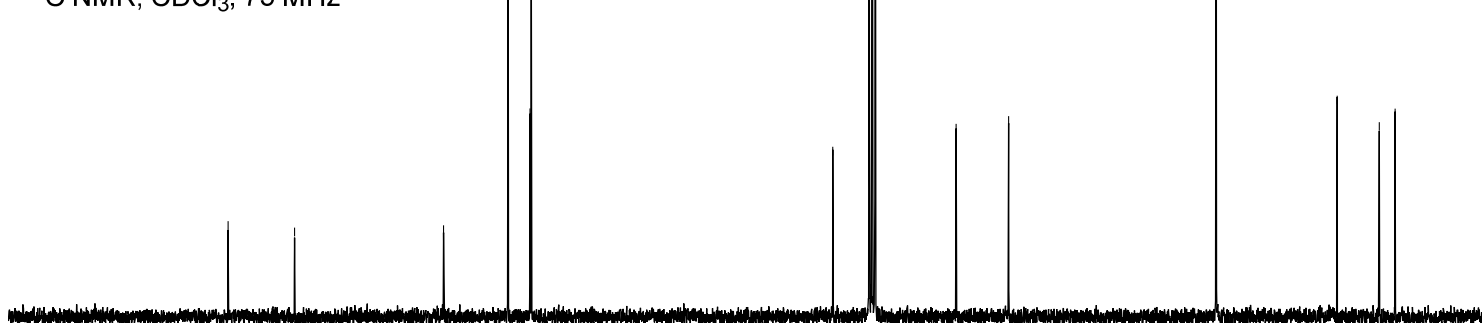

$\begin{array}{lllllllllll}00 & 190 & 180 & 170 & 160 & 150 & 140 & 130 & 120 & 110 & 100 \\ \mathrm{f} 1(\mathrm{ppm}) & 90\end{array}$ 

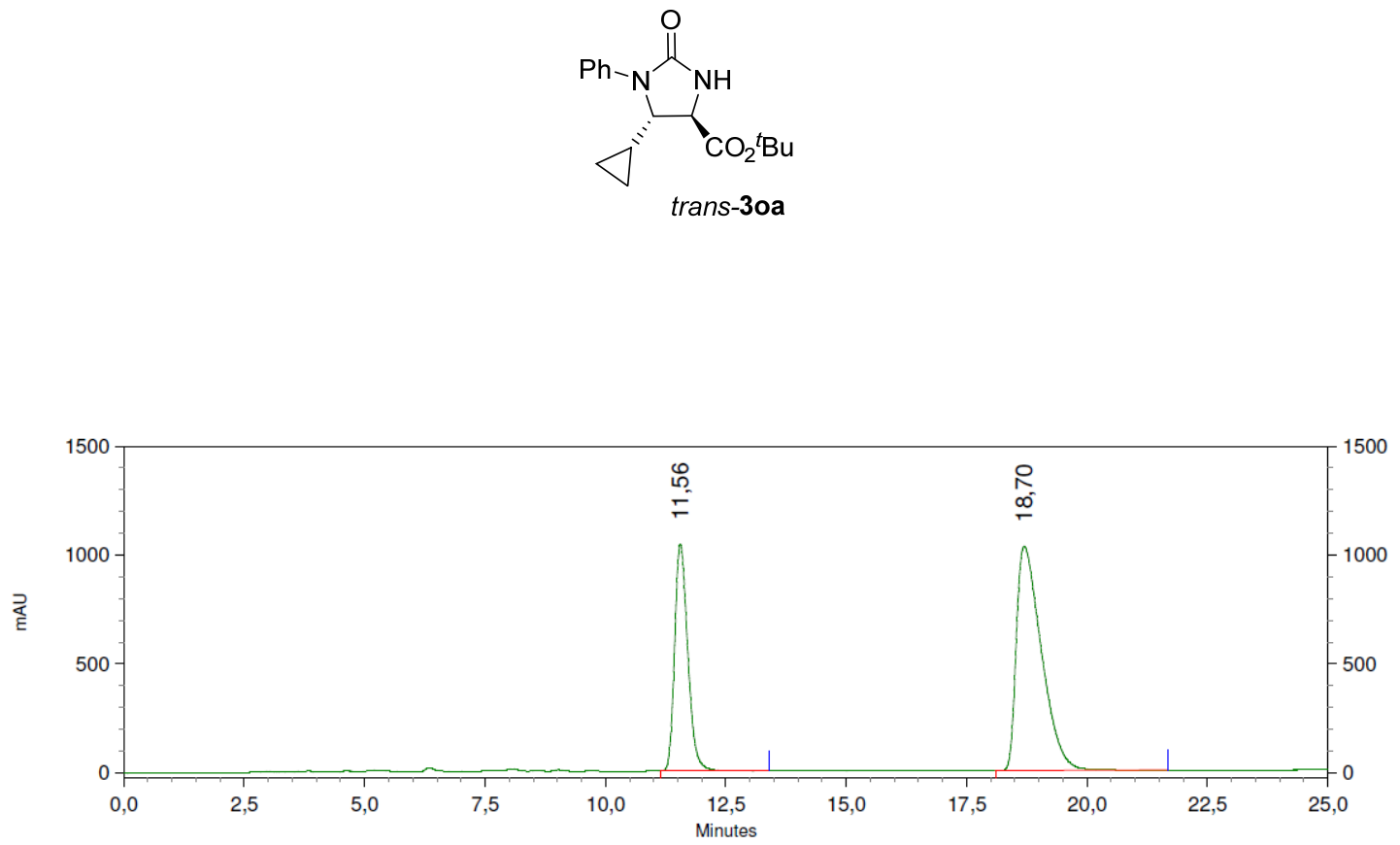

3: $244 \mathrm{~nm}, 4 \mathrm{~nm}$ Results Retention Time

Area Percent

$\begin{array}{rrr}11,56 & 78956991 & 34,650 \\ 18,70 & 148909923 & 65,350\end{array}$

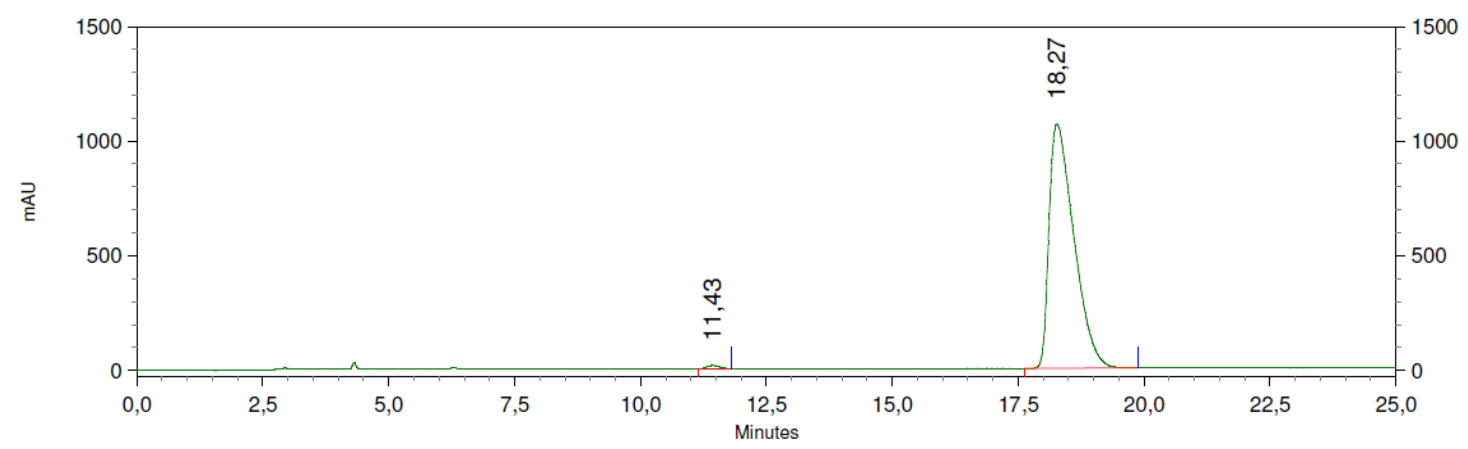

3: $244 \mathrm{~nm}, 4 \mathrm{~nm}$ Results Retention Time

11,43 Area Area Percent 18,27 1039532 0,708
99,292 

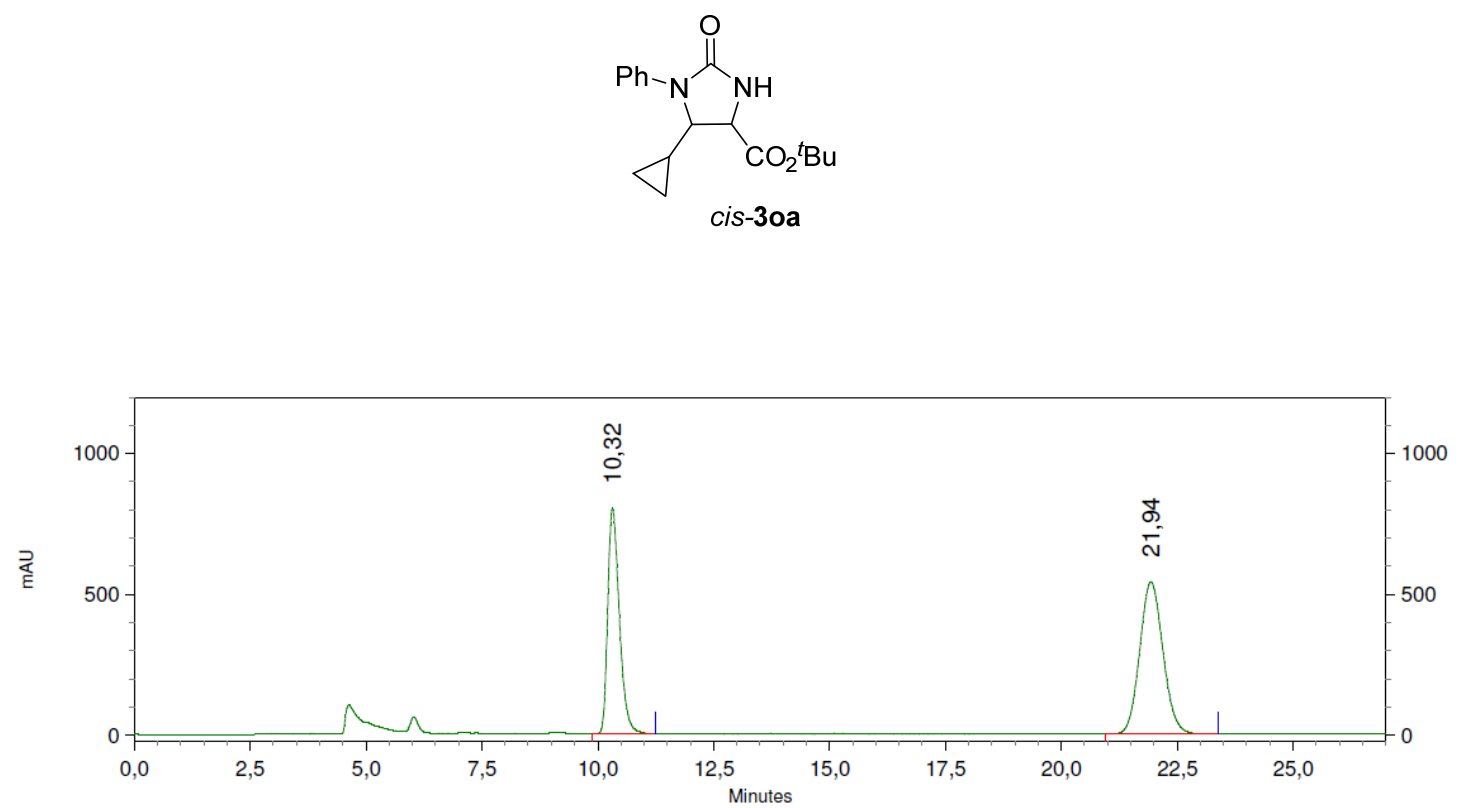

3: $244 \mathrm{~nm}, 4 \mathrm{~nm}$ Results Retention Time

$\begin{array}{lrr}10,32 & 55743154 & 42,558 \\ 21,94 & 75239095 & 57,442\end{array}$

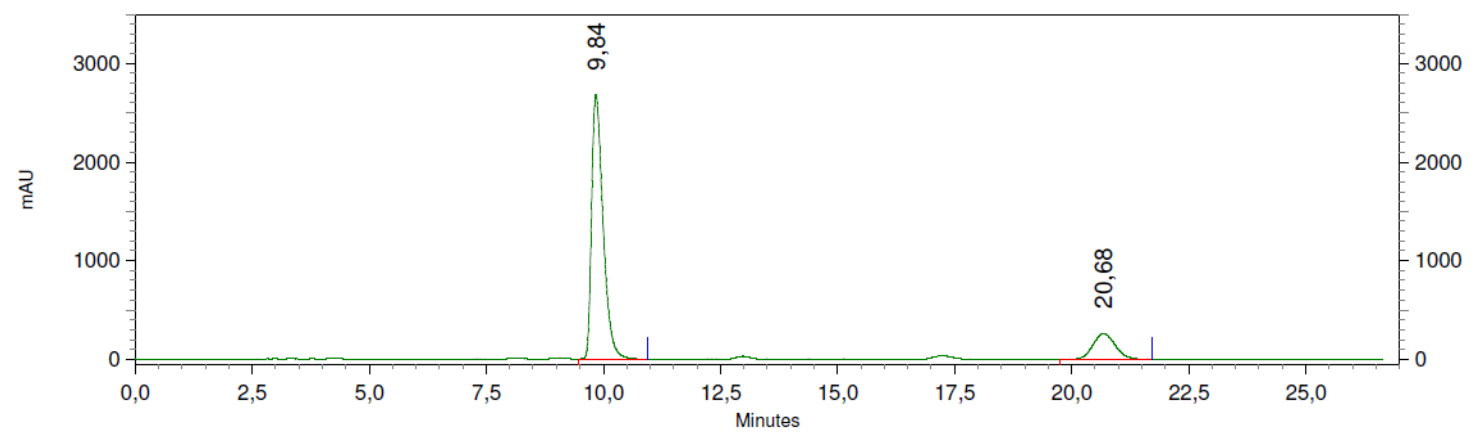

3: $244 \mathrm{~nm}, 4 \mathrm{~nm}$ Results Retention Time Area Area Percent

$\begin{array}{rrr}9,84 & 182186433 & 84,191 \\ 20,68 & 34211081 & 15,809\end{array}$




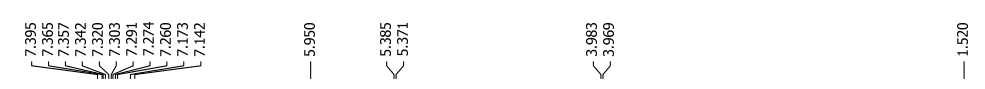

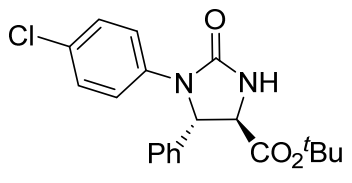

trans-(4R,5S)-3pa

${ }^{1} \mathrm{H} \mathrm{NMR}, \mathrm{CDCl}_{3}, 300 \mathrm{MHz}$

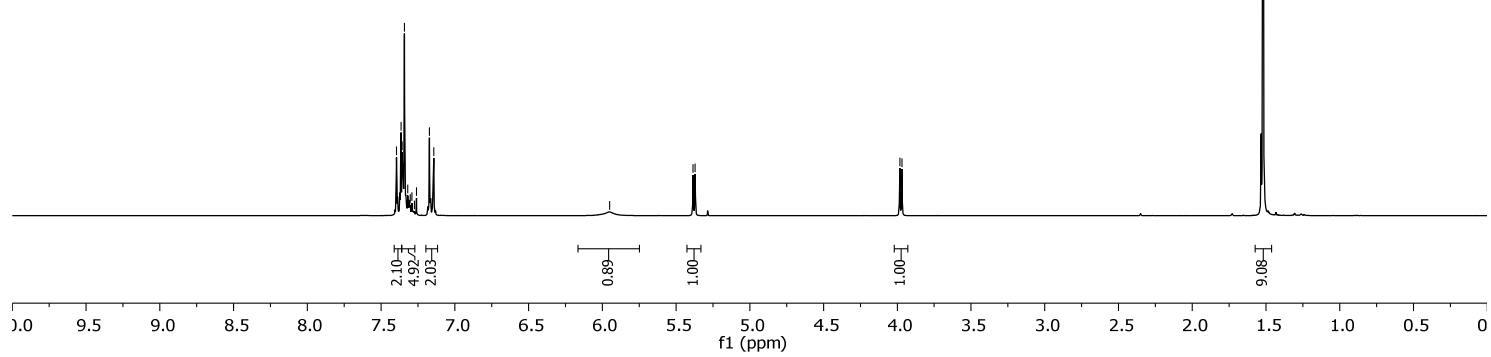

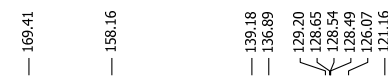

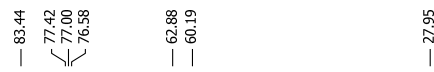

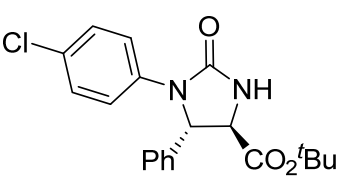

trans-(4R,5S)-3pa

${ }^{13} \mathrm{C} \mathrm{NMR}, \mathrm{CDCl}_{3}, 75 \mathrm{MHz}$

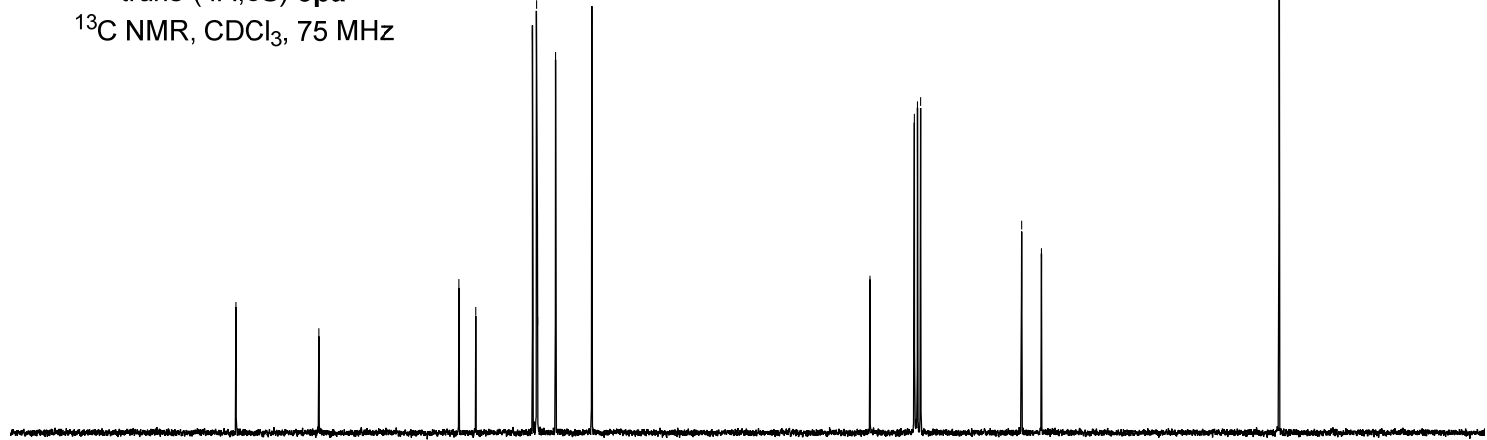

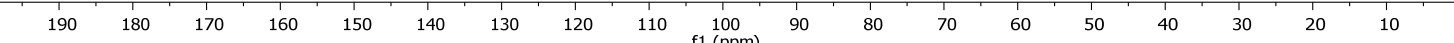




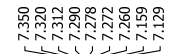

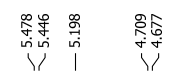

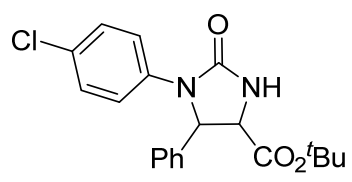

cis-3pa

${ }^{1} \mathrm{H} \mathrm{NMR}, \mathrm{CDCl}_{3}, 300 \mathrm{MHz}$

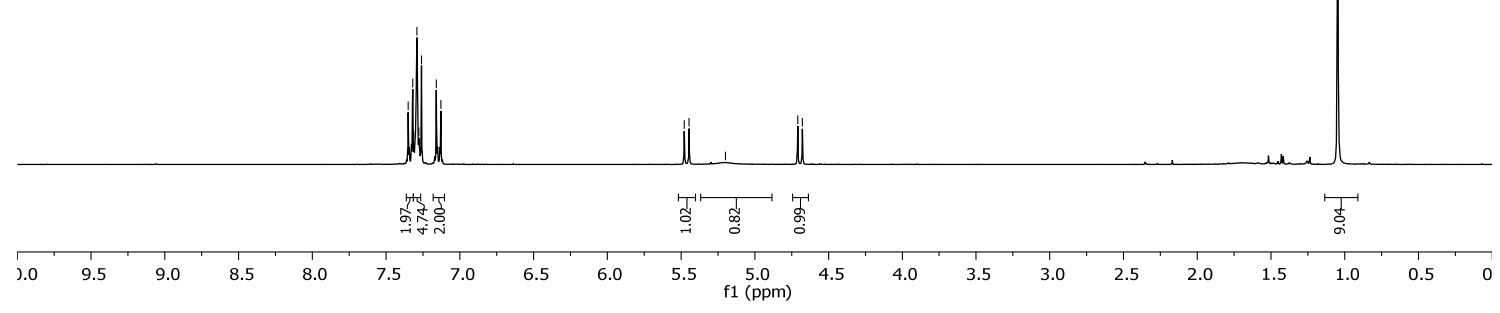

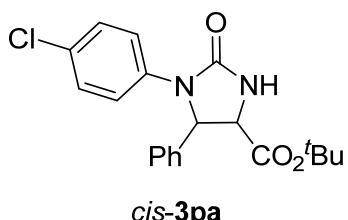

${ }^{13} \mathrm{C} \mathrm{NMR}, \mathrm{CDCl}_{3}, 75 \mathrm{MHz}$

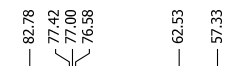

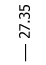

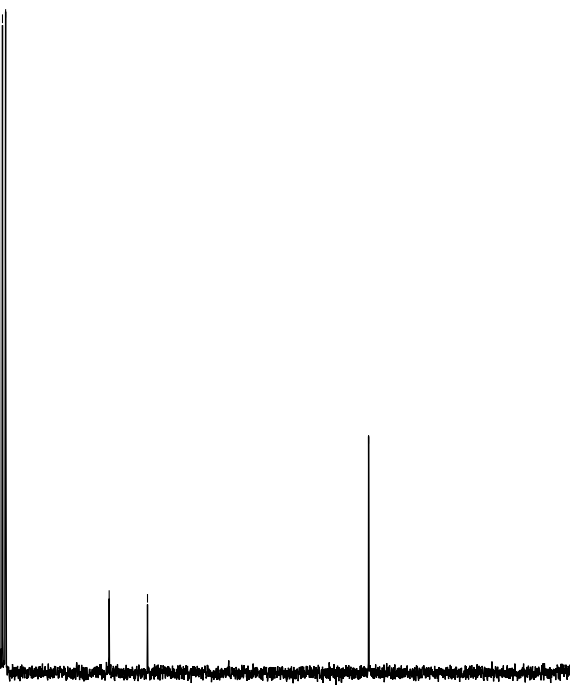

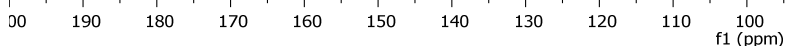




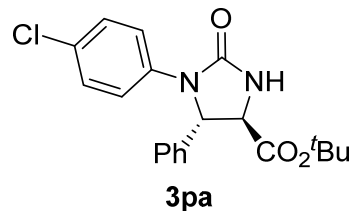

Racemic product (diastereomer mixture)

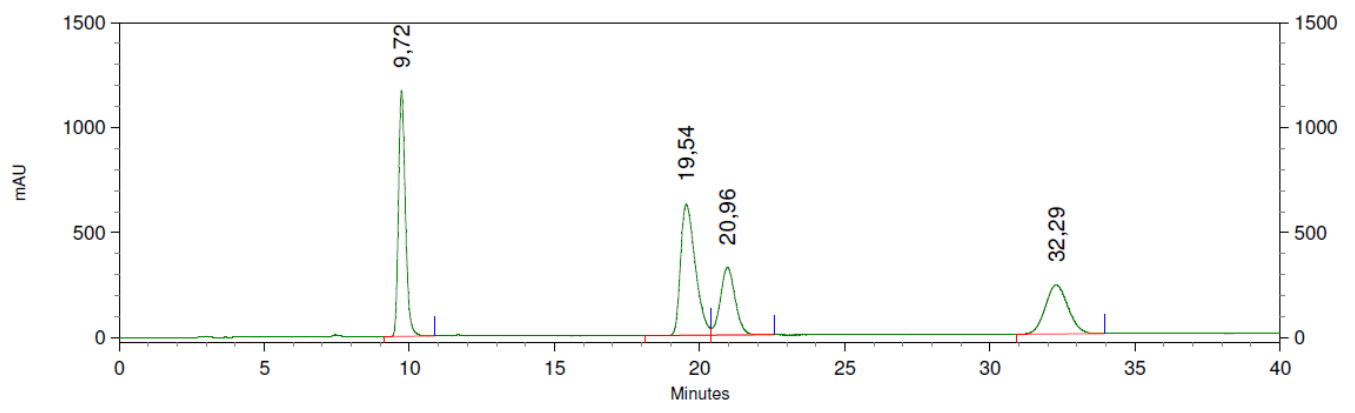

2: $250 \mathrm{~nm}, 4 \mathrm{~nm}$ Results Retention Time

$\begin{array}{rrr}9,72 & 77467030 & 29,577 \\ 19,54 & 88172253 & 33,665 \\ 20,96 & 45519168 & 17,379 \\ 32,29 & 50755563 & 19,379\end{array}$

trans-(4R,5S)-3pa

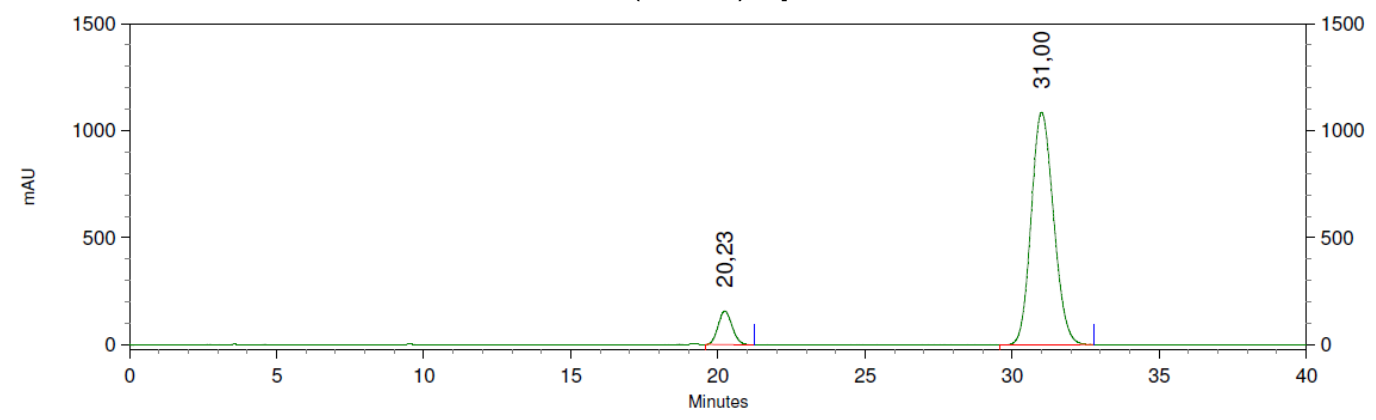

2: $250 \mathrm{~nm}, 4 \mathrm{~nm}$ Results Retention Time

\begin{tabular}{rrr} 
Time & Area & Area Percent \\
\hline 20,23 & 20663863 & 8,245 \\
31,00 & 229944034 & 91,755
\end{tabular}

cis-3pa

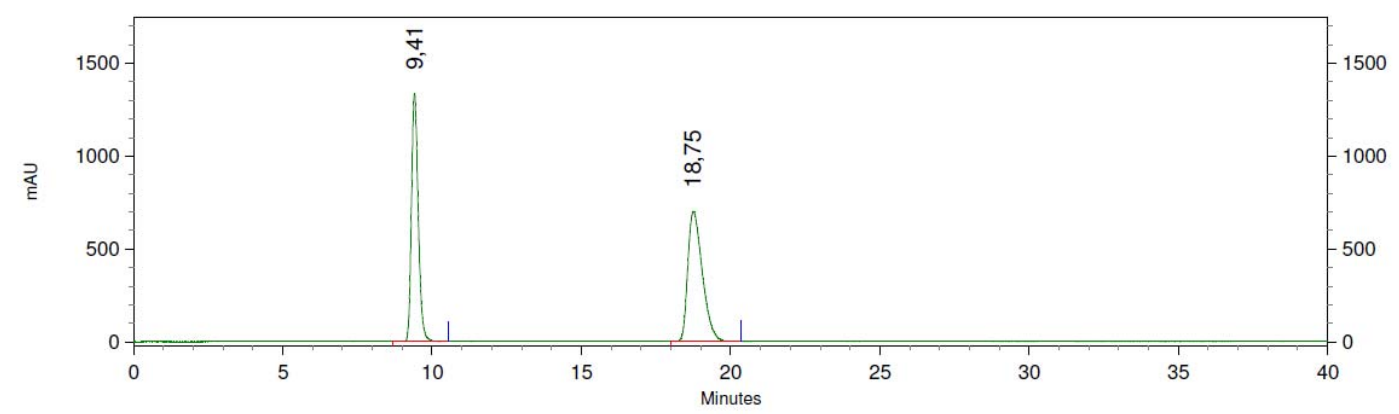

2: $250 \mathrm{~nm}, 4 \mathrm{~nm}$ Results

Retention Time

9,41
18,75 


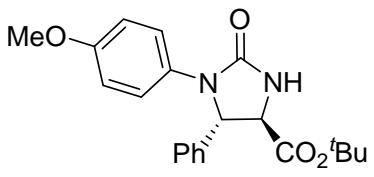

trans-(4R,5S)-3qa

${ }^{1} \mathrm{H} \mathrm{NMR}, \mathrm{CDCl}_{3}, 300 \mathrm{MHz}$
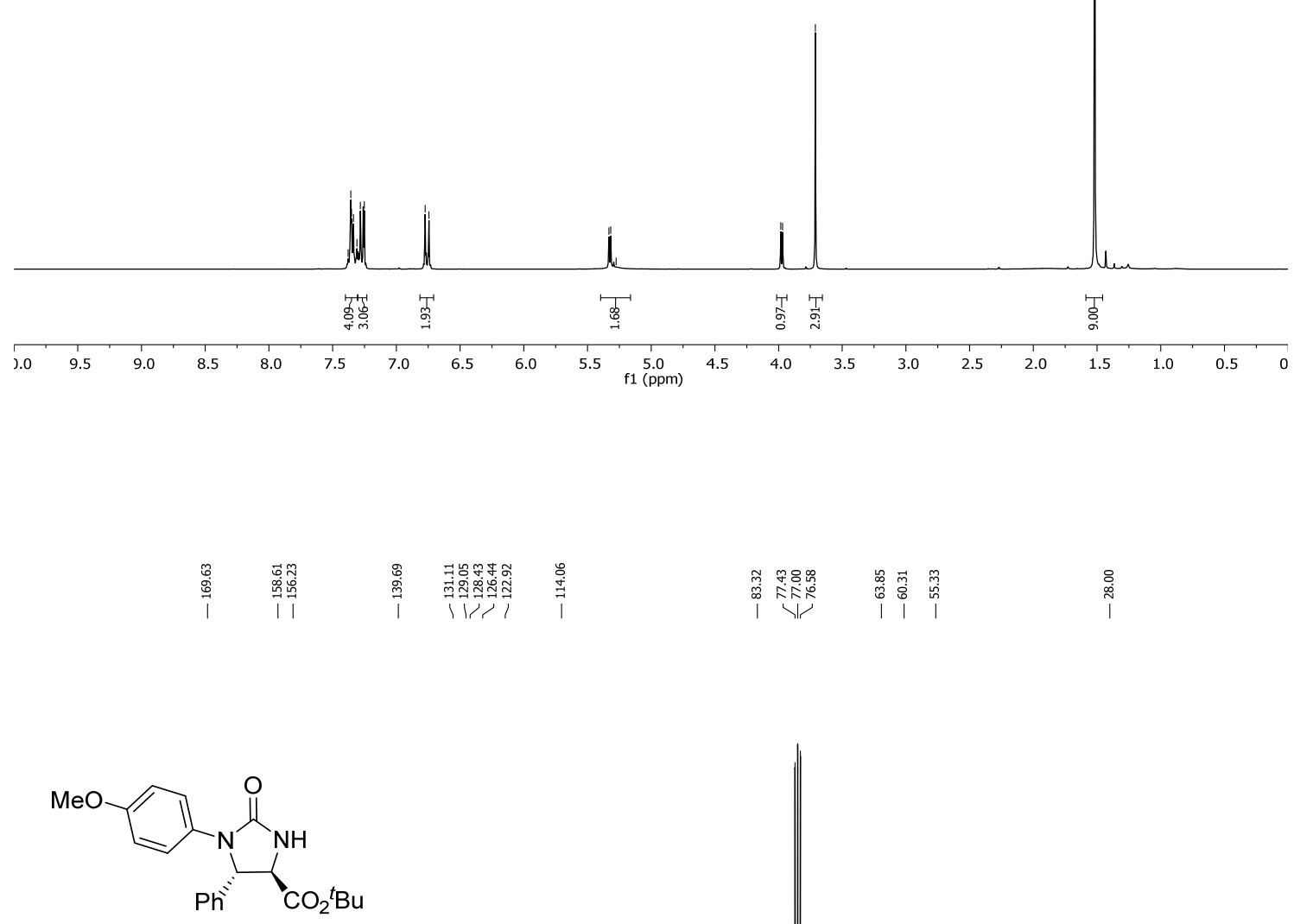

trans-(4R,5S)-3qa

${ }^{13} \mathrm{C} \mathrm{NMR}, \mathrm{CDCl}_{3}, 75 \mathrm{MHz}$

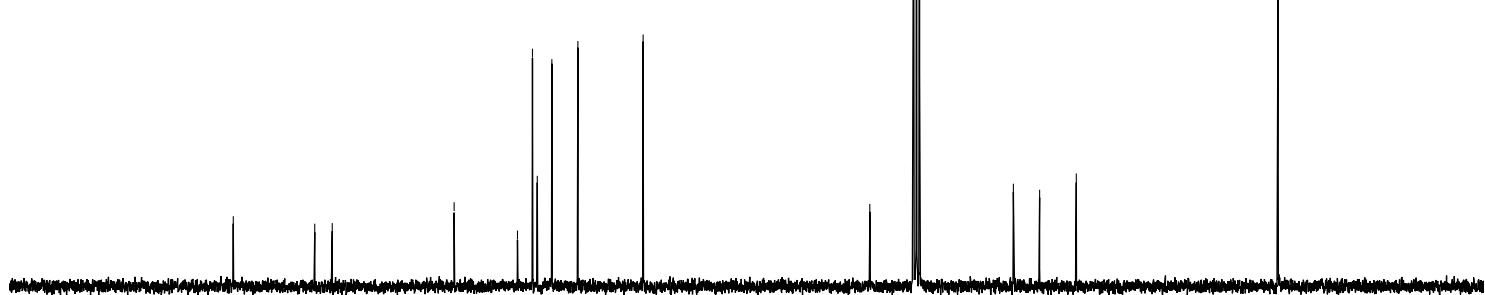

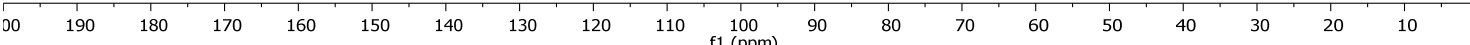




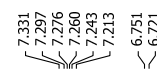

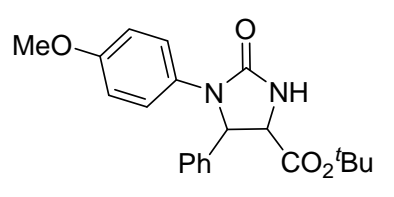

cis-3qa

${ }^{1} \mathrm{H} \mathrm{NMR}, \mathrm{CDCl}_{3}, 300 \mathrm{MHz}$

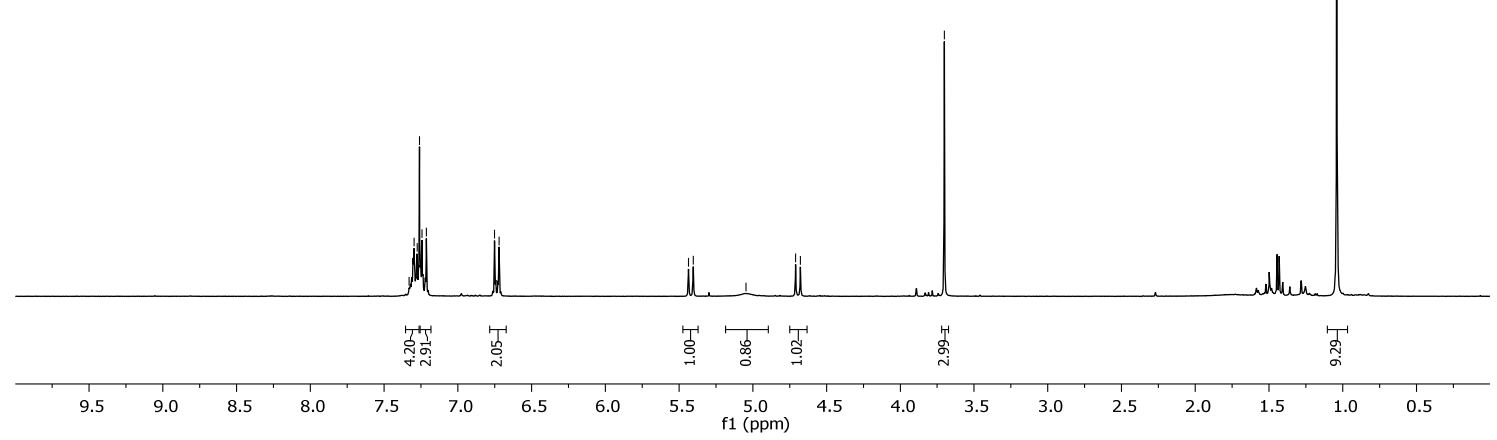

0
0
0

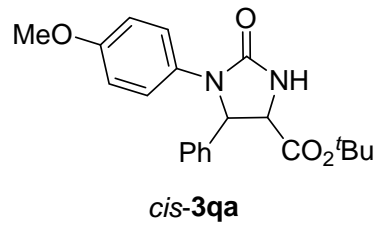

${ }^{13} \mathrm{C} \mathrm{NMR}, \mathrm{CDCl}_{3}, 75 \mathrm{MHz}$

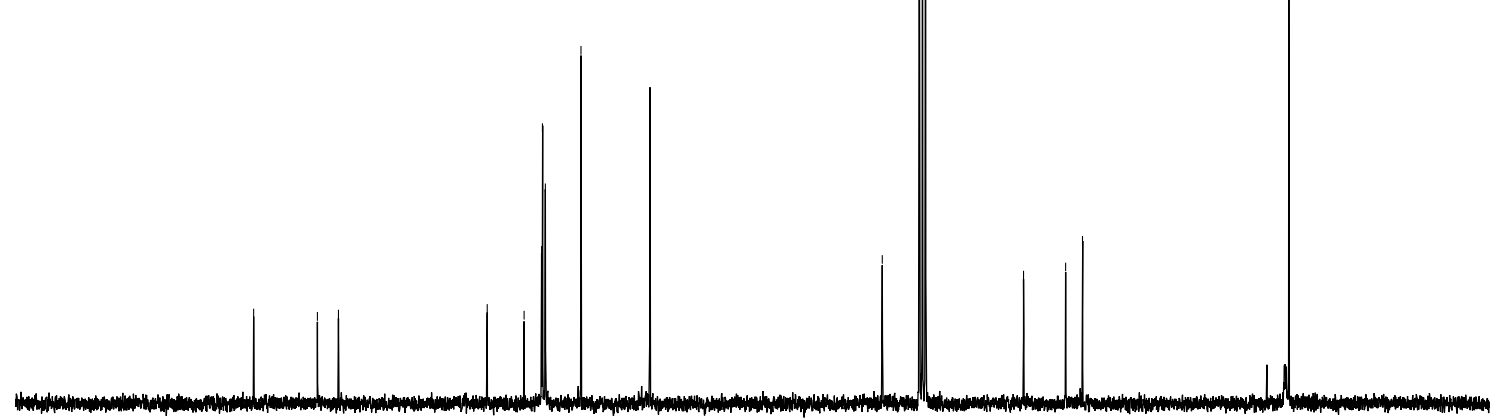

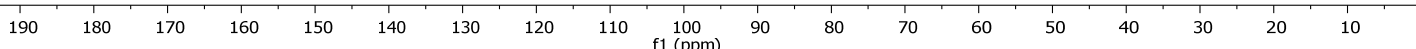




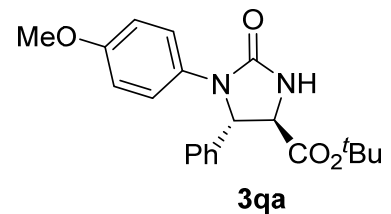

Racemic product (diastereomer mixture)

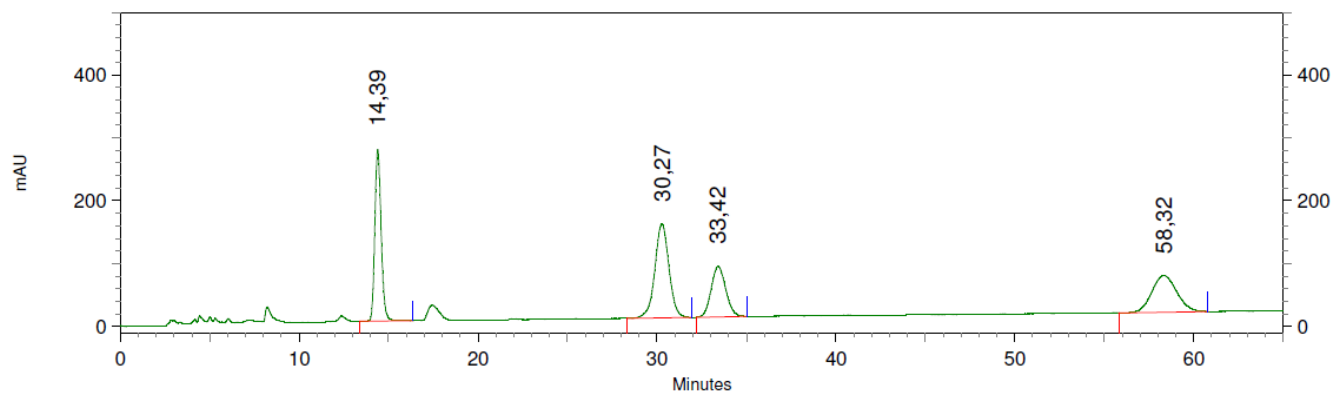

3: $244 \mathrm{~nm}, 4 \mathrm{~nm}$ Results

\begin{tabular}{rrr} 
Retention Time & Area & Area Percent \\
\hline 14,39 & 26804009 & 26,725 \\
30,27 & 32711839 & 32,616 \\
33,42 & 18185168 & 18,132 \\
58,32 & 22594033 & 22,528
\end{tabular}

trans-(4R,5S)-3qa

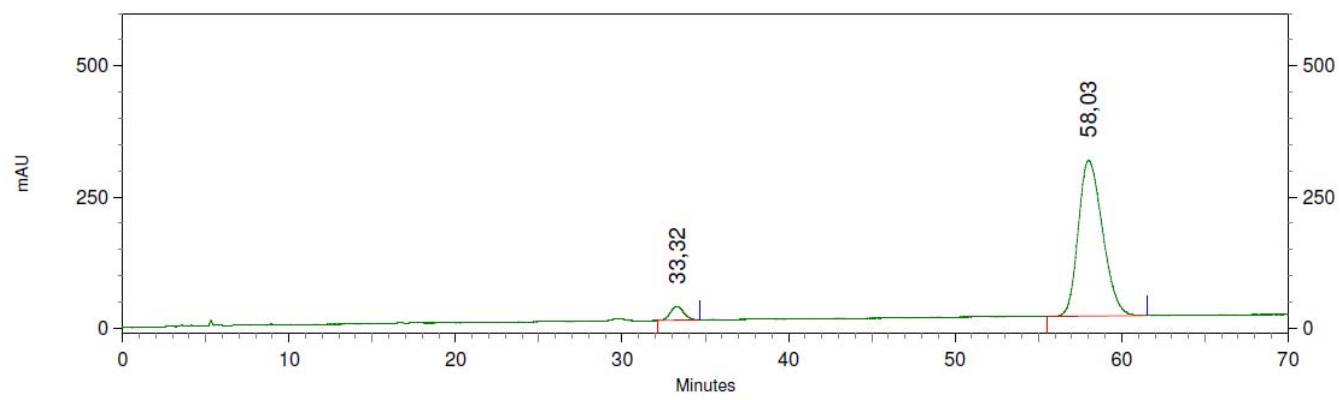

2: 242 nm, 4 nm Results Retention Time

$\begin{array}{rrr}\text { 33, 32 } & \text { Area } & 4,642 \\ 58,03 & 120212242 & 95,358\end{array}$

cis-3qa

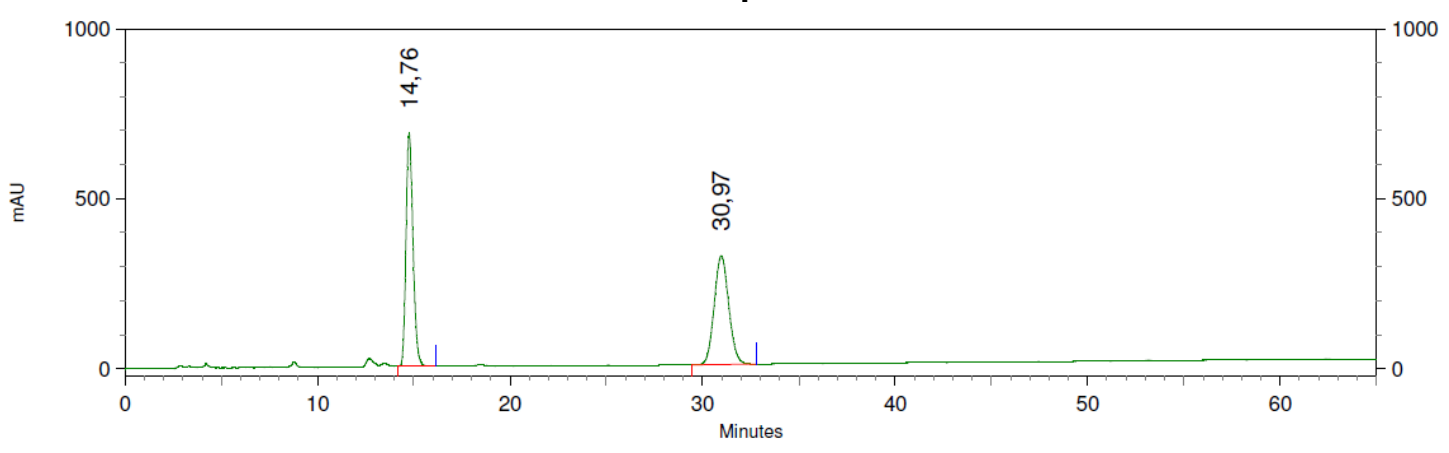

3: 244 nm, 4 nm Results Retention Time

14,76

Area

Area Percent

30,97 

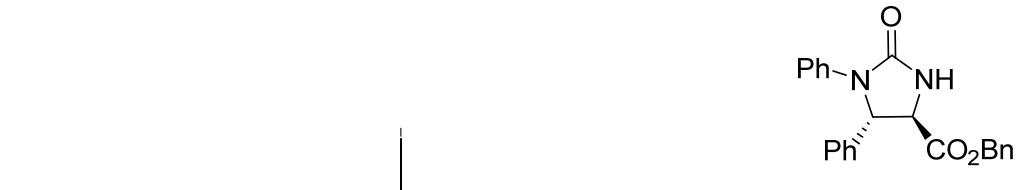

trans-(4R,5S)-3ab

${ }^{1} \mathrm{H} \mathrm{NMR}, \mathrm{CDCl}_{3}, 300 \mathrm{MHz}$

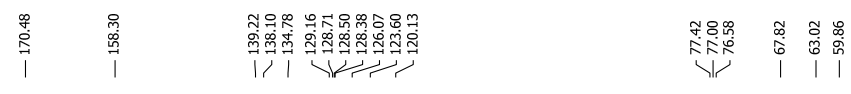

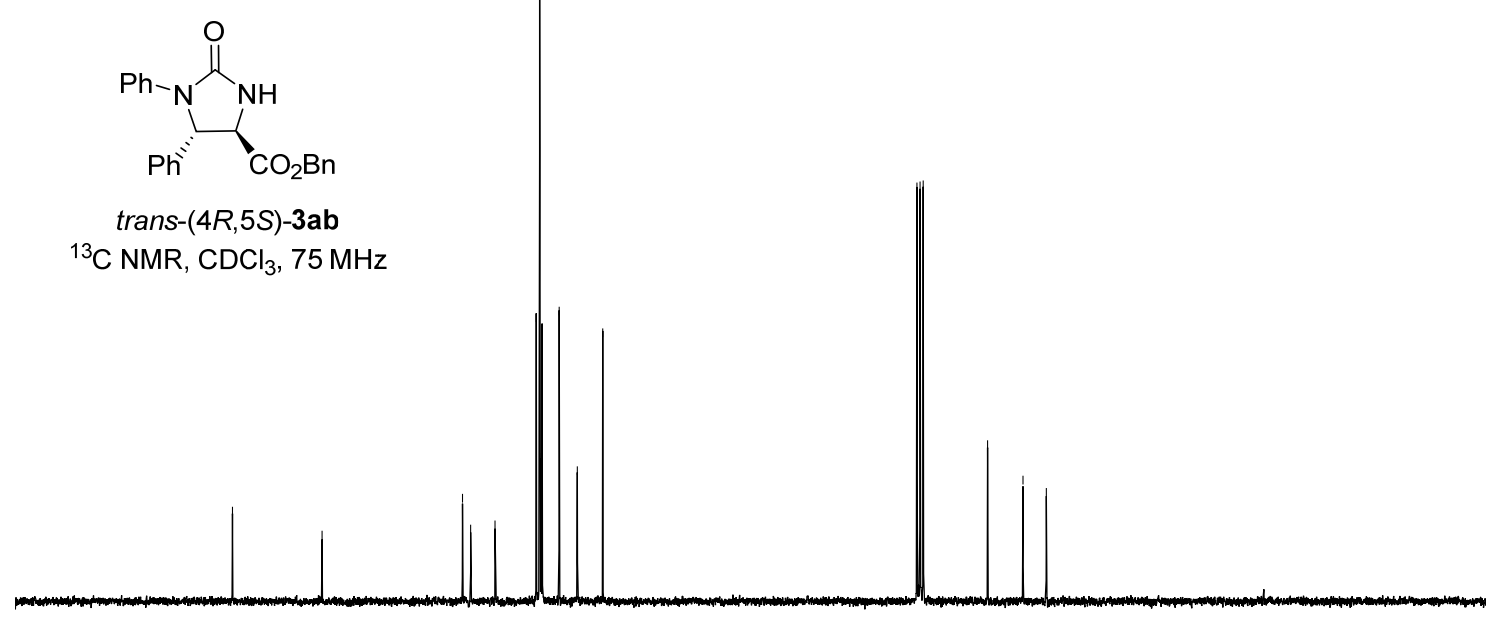

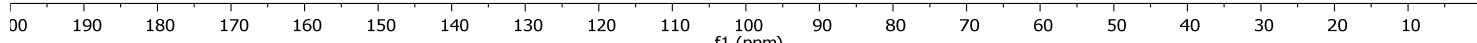




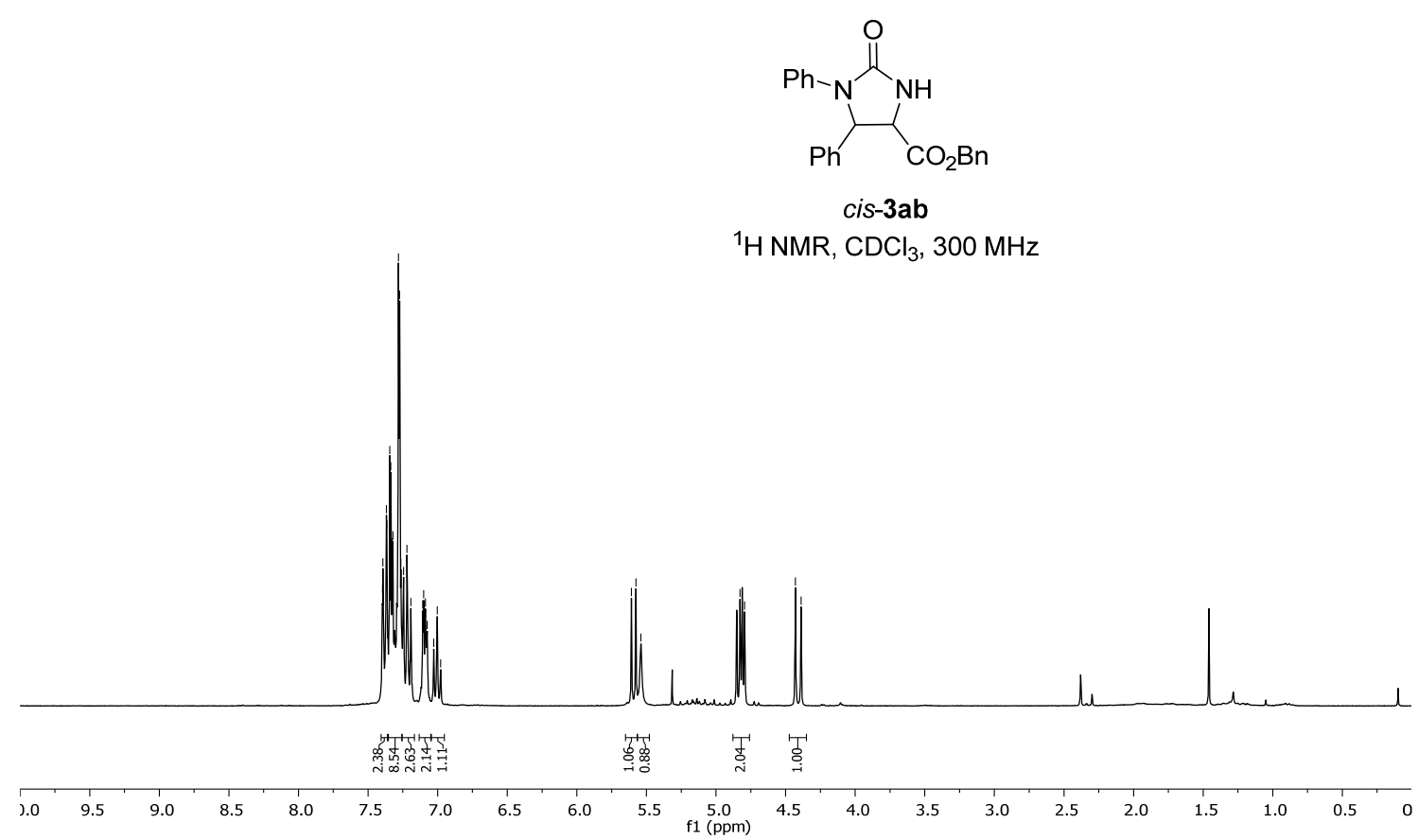

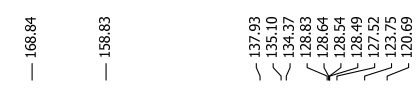

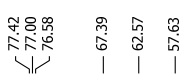

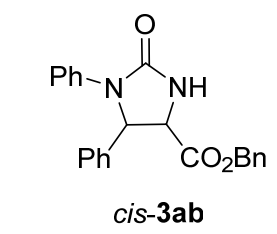

${ }^{13} \mathrm{C} \mathrm{NMR}, \mathrm{CDCl}_{3}, 75 \mathrm{MHz}$

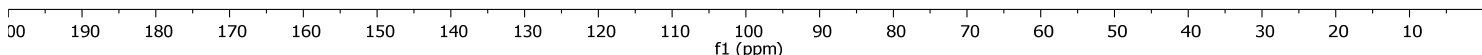




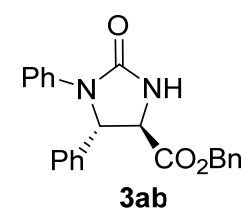

Racemic product (diastereomer mixture)

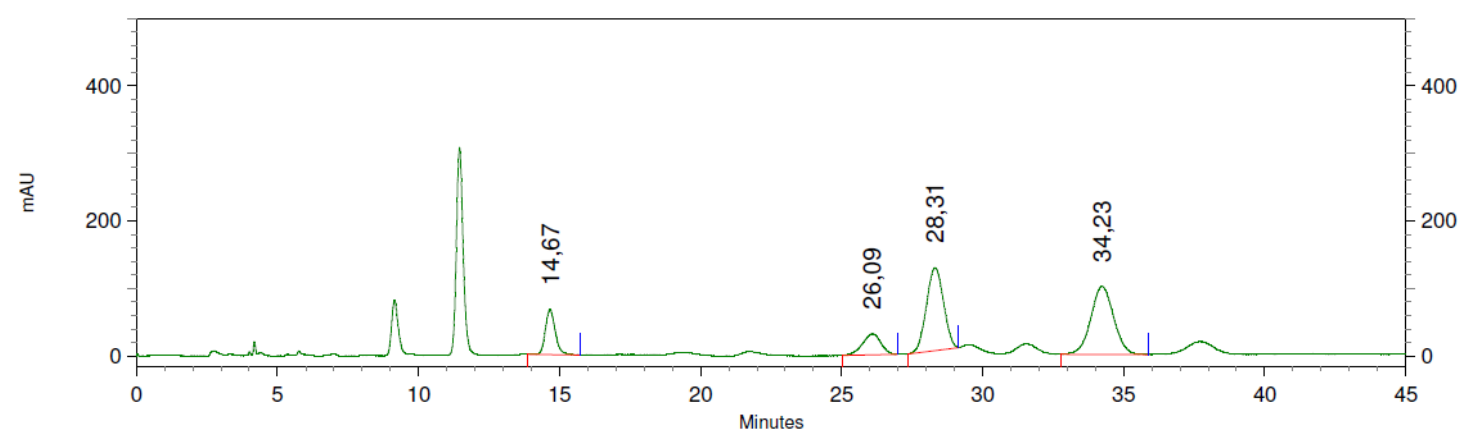

2: $241 \mathrm{~nm}, 4$ nm Results

\begin{tabular}{rrr} 
Retention Time & Area & Area Percent \\
\hline 14,67 & 6570126 & 11,846 \\
26,09 & 5624142 & 10,141 \\
28,31 & 20639900 & 37,215 \\
34,23 & 22627008 & 40,798
\end{tabular}

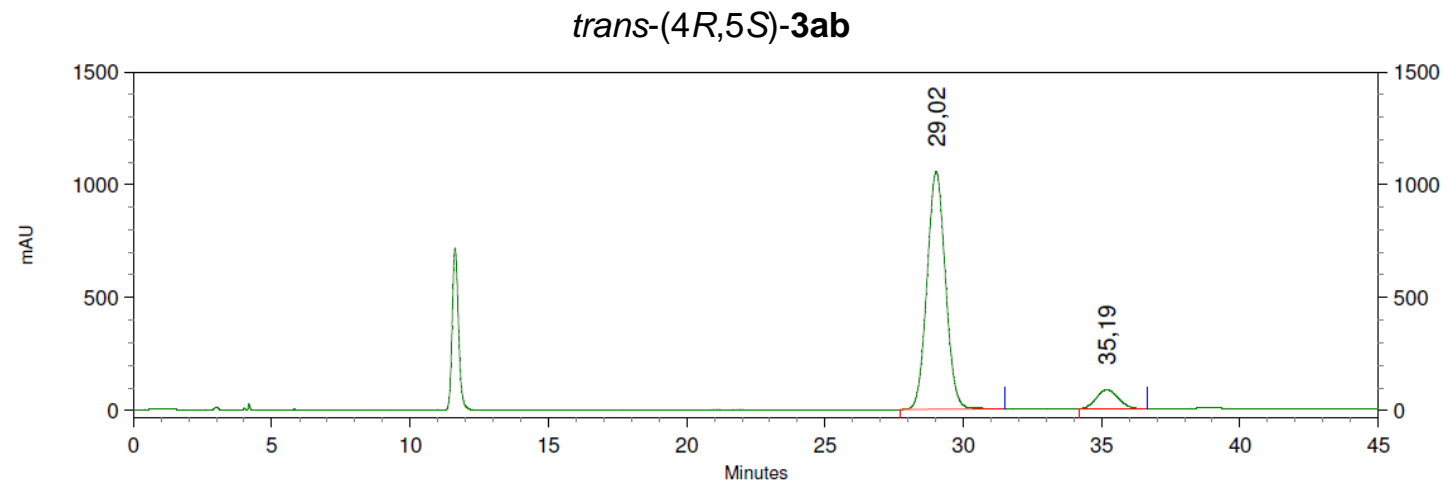

2: $241 \mathrm{~nm}, 4 \mathrm{~nm}$ Results Retention Time

$\begin{array}{rrr}29,02 & 194966667 & 91,172 \\ 35,19 & 18879001 & 8,828\end{array}$

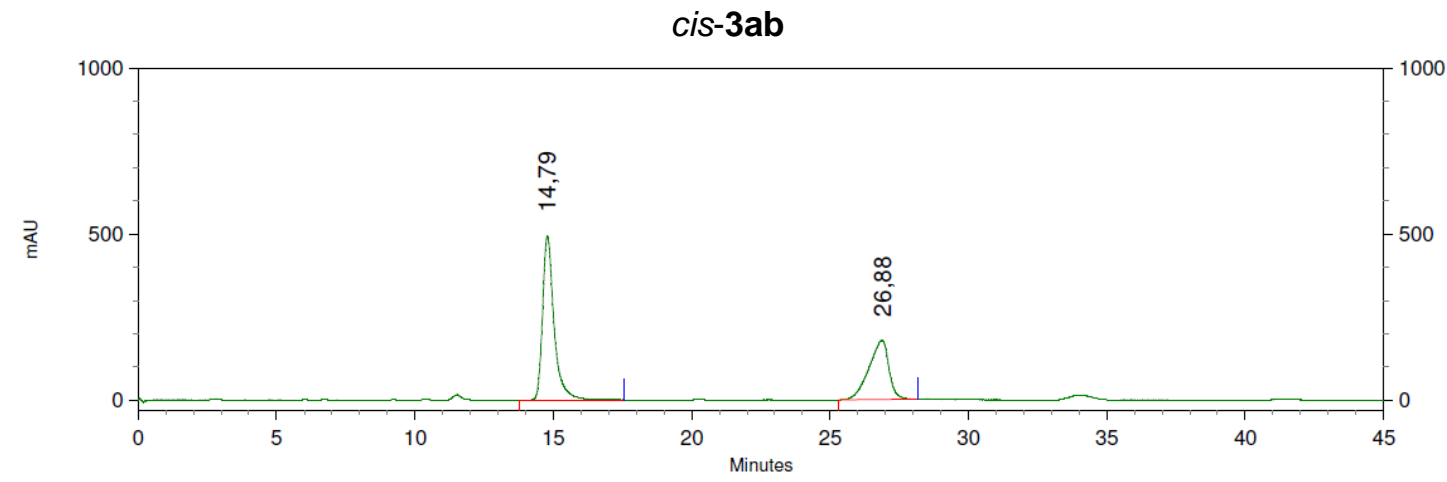

2: $241 \mathrm{~nm}, 4 \mathrm{~nm}$ Results Retention Time 14,79
26,88 


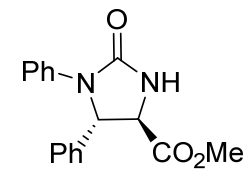

trans-(4R,5S)-3ac

${ }^{1} \mathrm{H} \mathrm{NMR}, \mathrm{CDCl}_{3}, 300 \mathrm{MHz}$

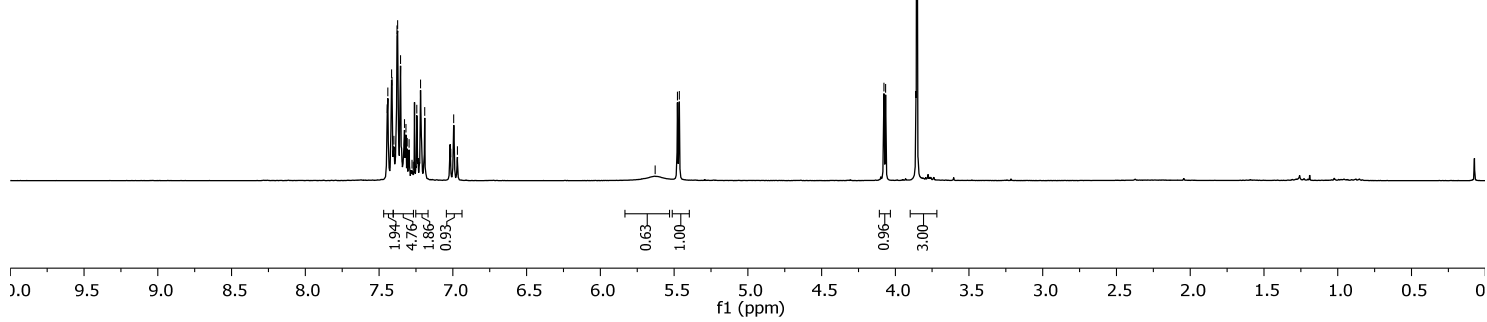

年

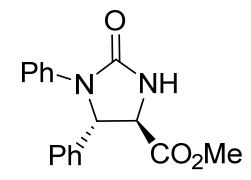

trans-(4R,5S)-3ac

${ }^{13} \mathrm{C} \mathrm{NMR}, \mathrm{CDCl}_{3}, 75 \mathrm{MHz}$

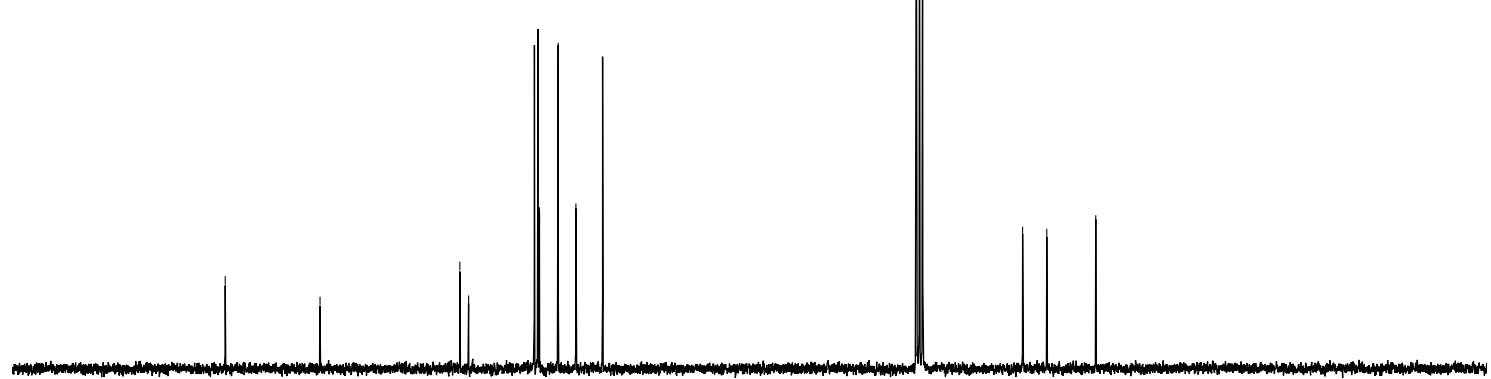

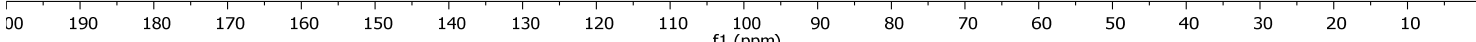




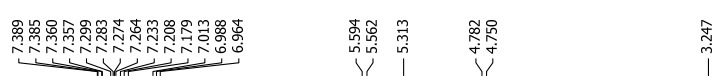

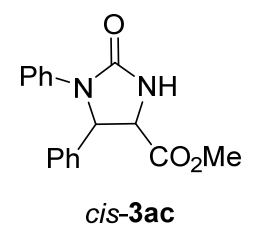

${ }^{1} \mathrm{H} \mathrm{NMR}, \mathrm{CDCl}_{3}, 300 \mathrm{MHz}$

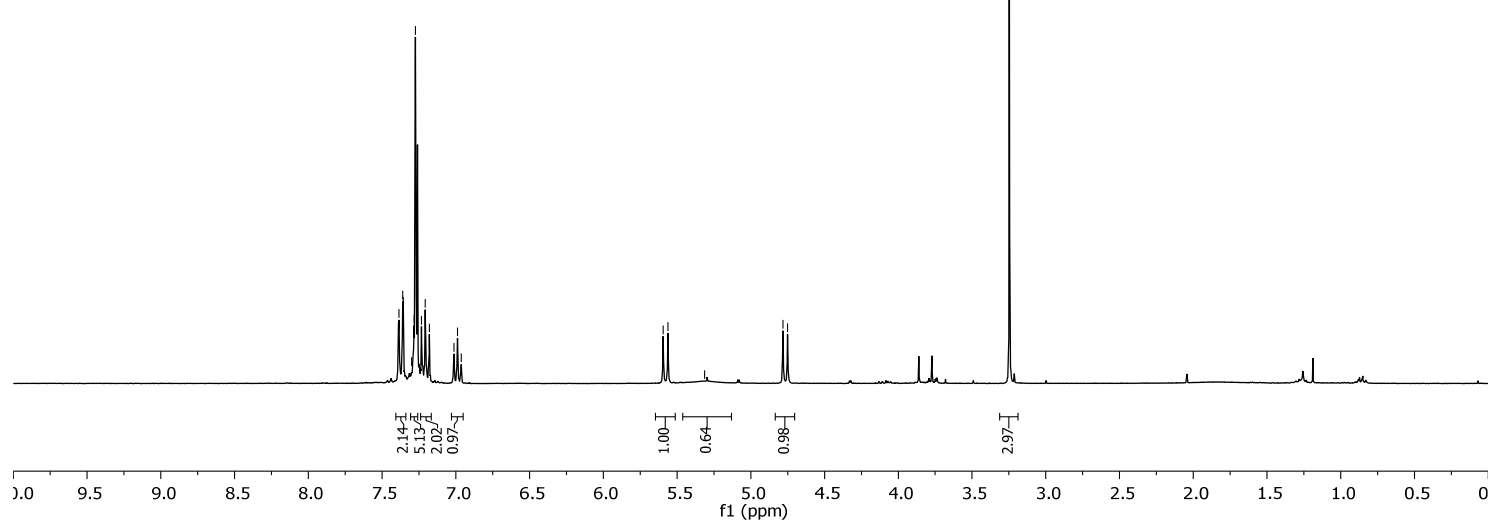

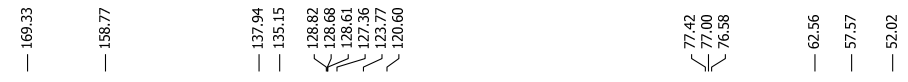

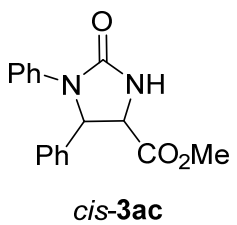

${ }^{13} \mathrm{C} \mathrm{NMR}, \mathrm{CDCl}_{3}, 75 \mathrm{MHz}$

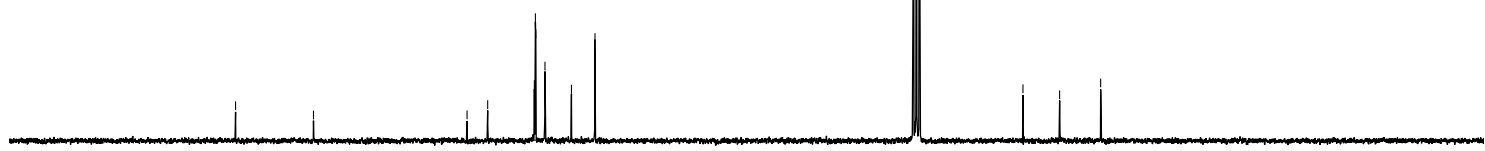

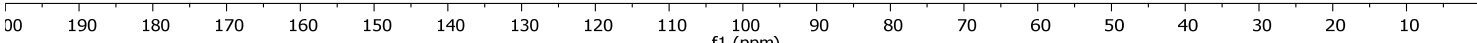




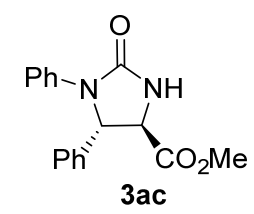

Racemic product (diastereomer mixture)

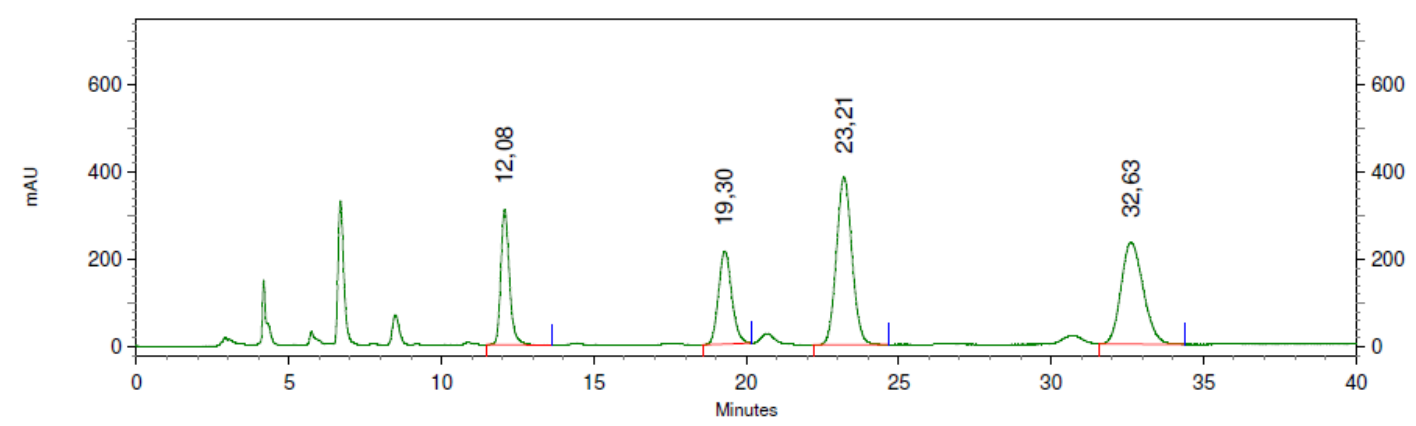

6: $242 \mathrm{~nm}, 4 \mathrm{~nm}$ Results Retention Time

12,08

19,30

23,21

32,63

24313007
25742369
54392768
47171612

Area Percent

16,036
16,978

35,874

31,112

trans-(4R,5S)-3ac

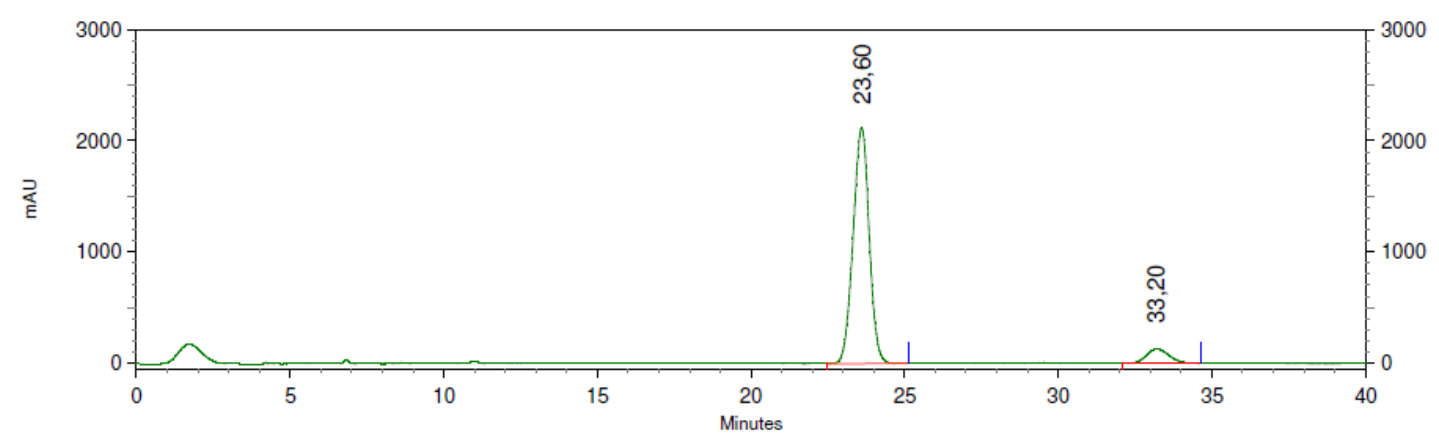

6: 242 nm, 4 nm Results Retention Time

$\begin{array}{rrr} & \text { Area } & \text { Area } \\ 23,60 & 309238470 & 92,054 \\ 33,20 & 26694331 & 7,946\end{array}$

cis-3s

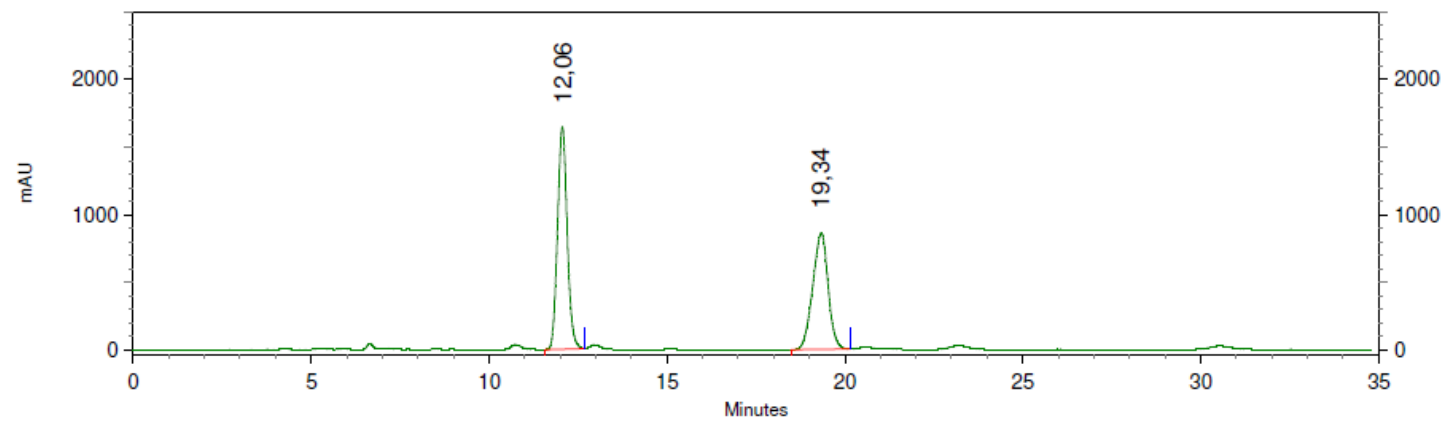

6: $242 \mathrm{~nm}, 4 \mathrm{~nm}$ Results

Retention Time

12,06
19,34

Area

Area Percent

123813417

54,431 


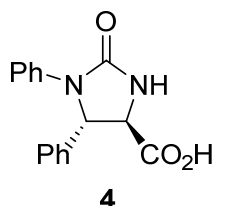

${ }^{1} \mathrm{H} \mathrm{NMR}, \mathrm{CDCl}_{3}, 300 \mathrm{MHz}$
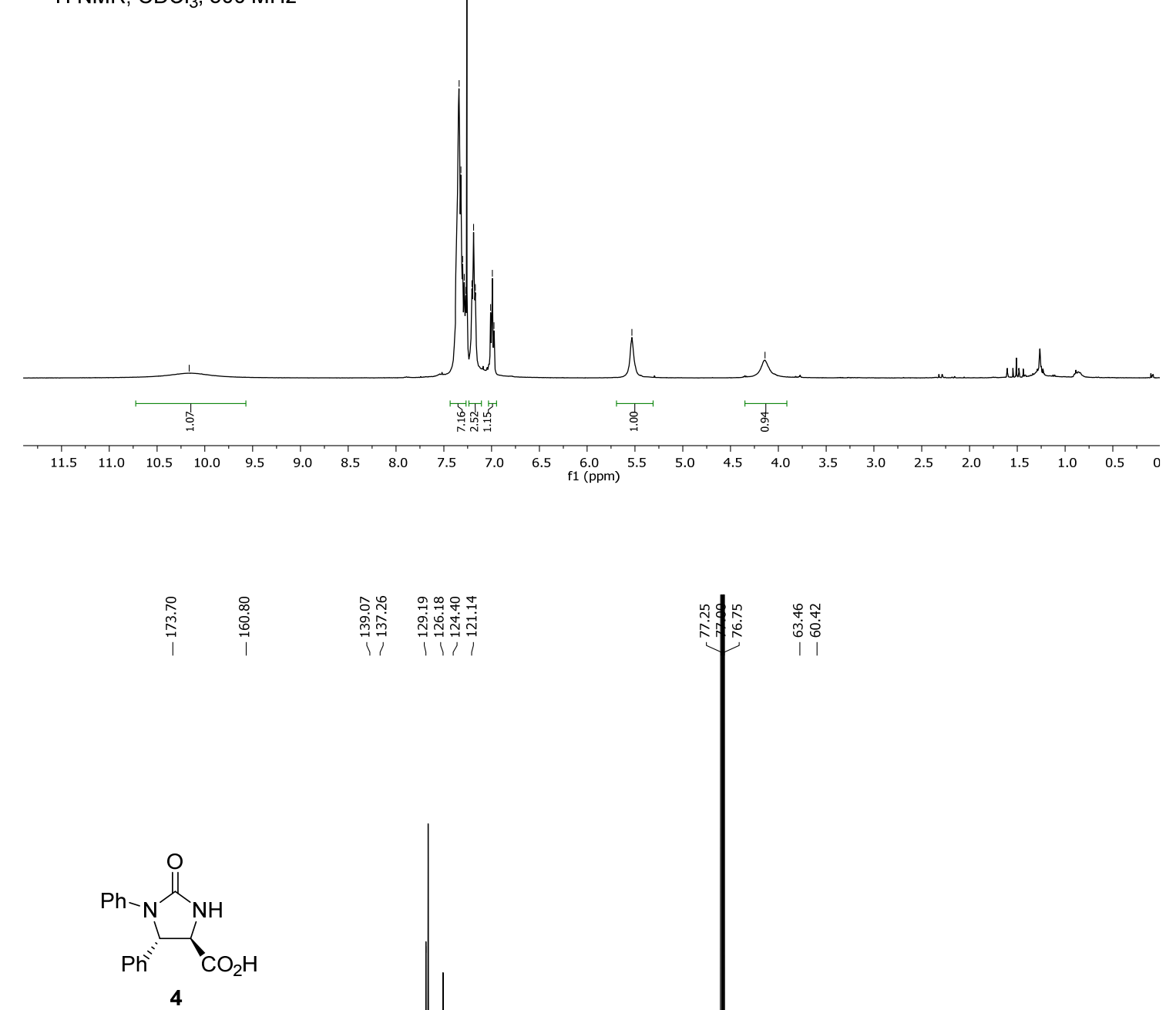

${ }^{13} \mathrm{C} \mathrm{NMR}, \mathrm{CDCl}_{3}, 75 \mathrm{MHz}$

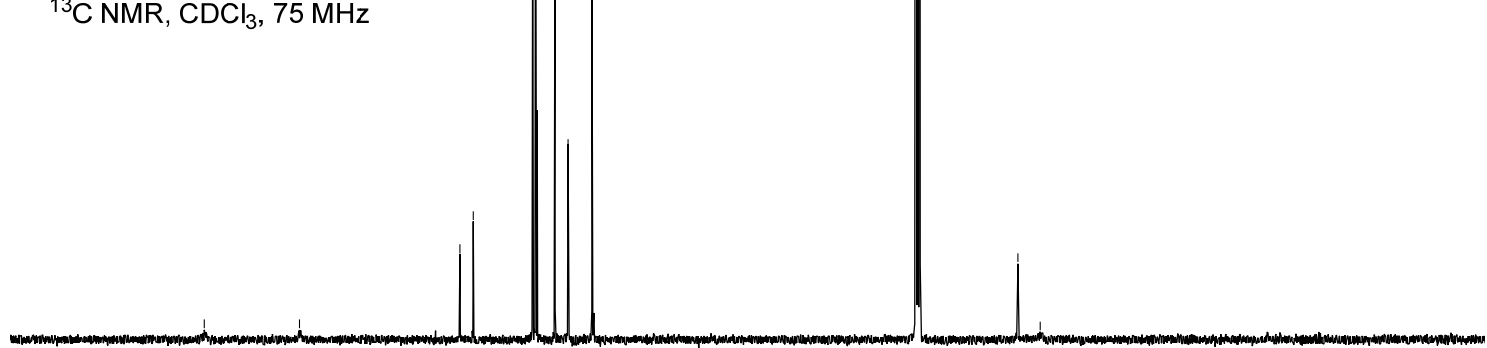

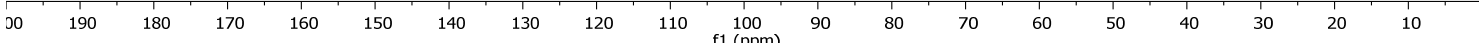




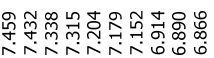

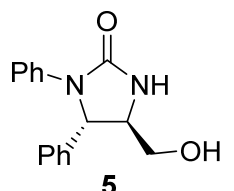

${ }^{1} \mathrm{H}$ NMR, DMSO-d6, $300 \mathrm{MHz}$

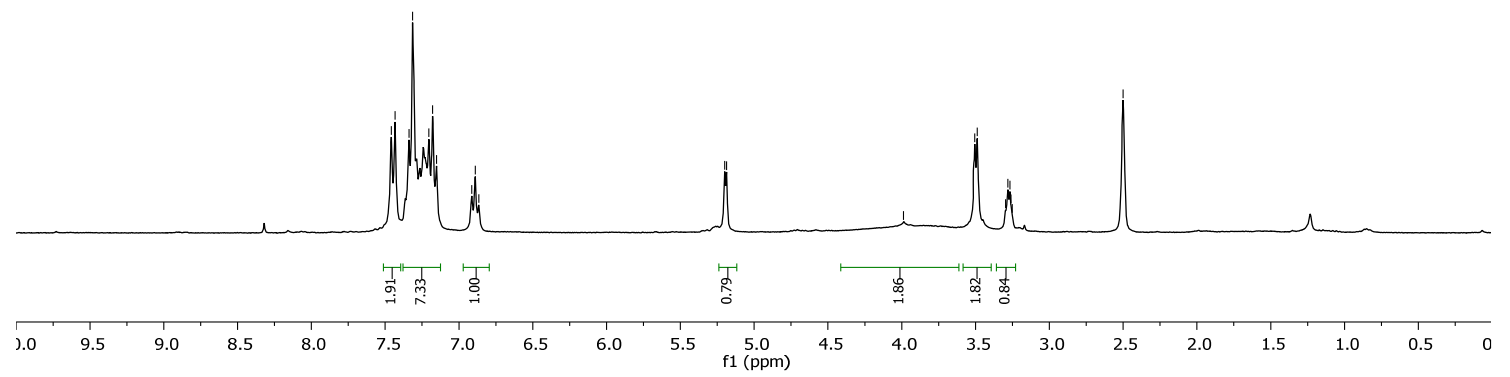

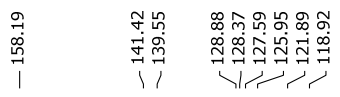

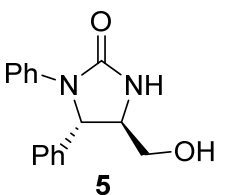

${ }^{13} \mathrm{C}$ NMR, DMSO-d6, $75 \mathrm{MHz}$

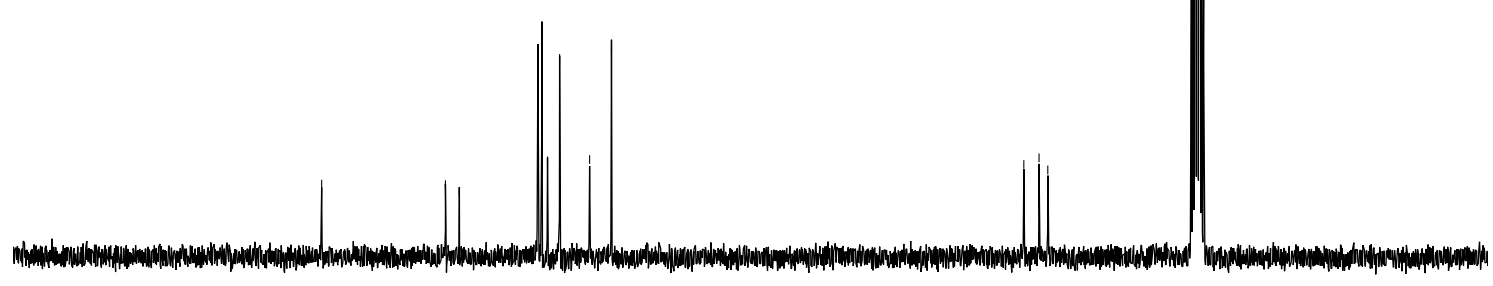

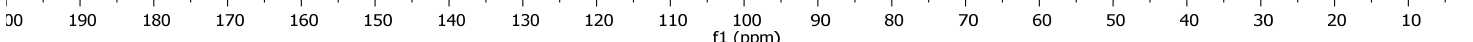



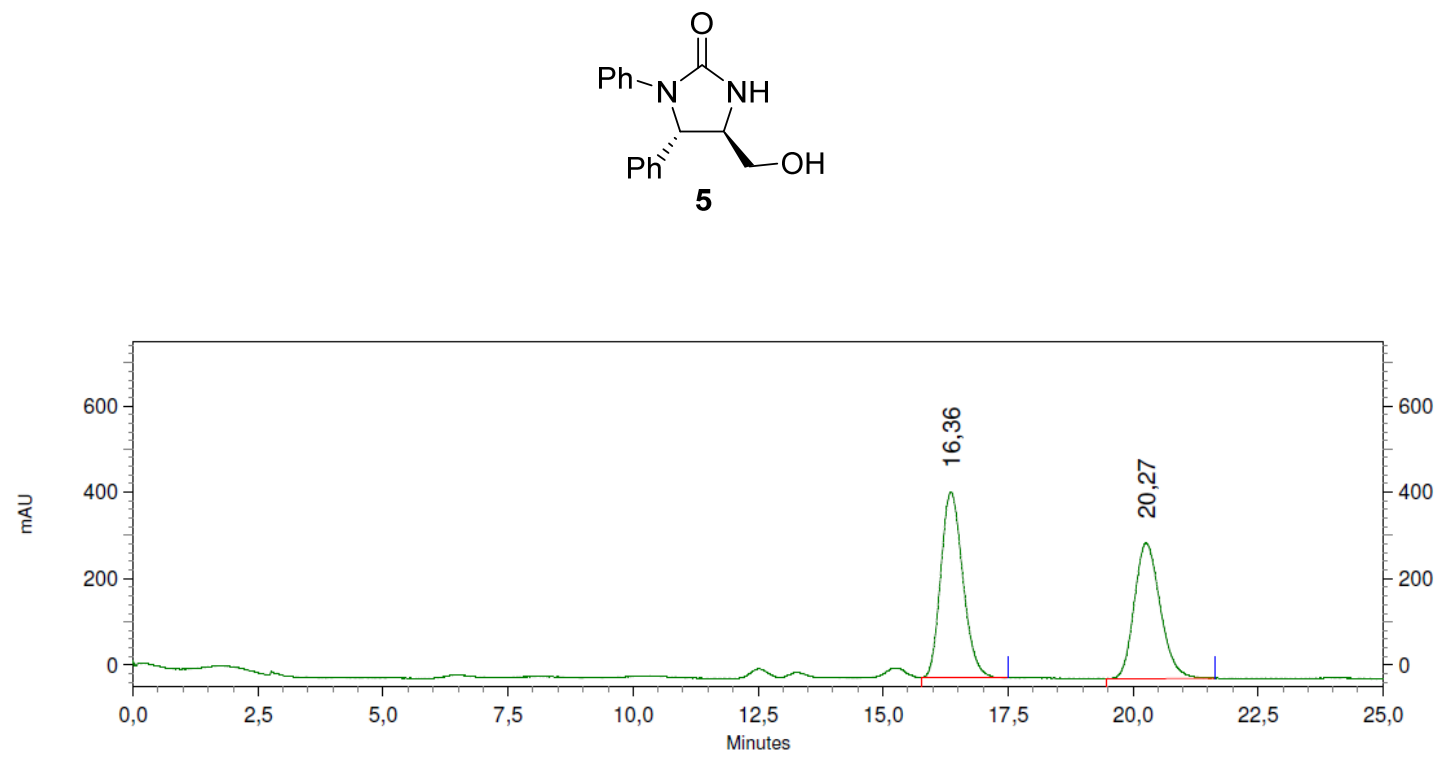

14: $244 \mathrm{~nm}, 4 \mathrm{~nm}$ Results

\begin{tabular}{rrr} 
Retention Time & Area & Area Percent \\
\hline 16,36 & 53085023 & 53,837 \\
20,27 & 45519124 & 46,163
\end{tabular}

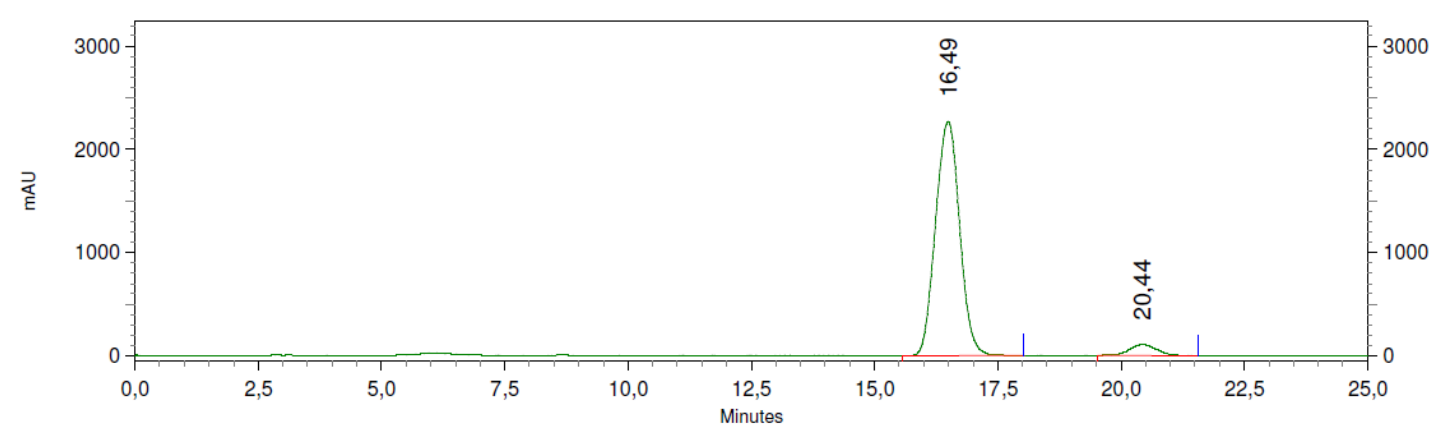

14: $244 \mathrm{~nm}, 4 \mathrm{~nm}$

Results

Retention Time

Area

Area Percent

$\begin{array}{rrr}16,49 & 308774878 & 94,891 \\ 20,44 & 16623283 & 5,109\end{array}$




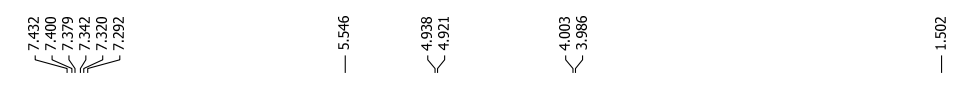

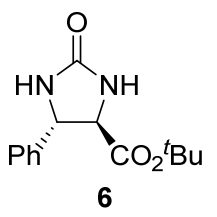

${ }^{1} \mathrm{H} \mathrm{NMR}, \mathrm{CDCl}_{3}, 300 \mathrm{MHz}$

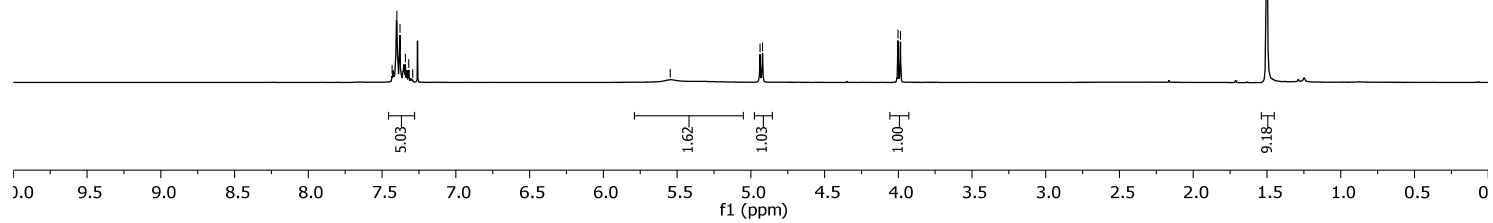

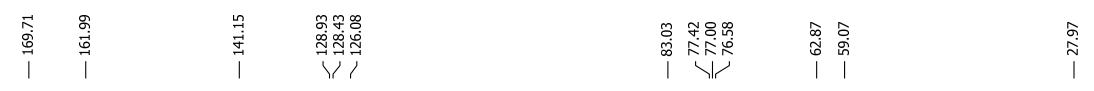

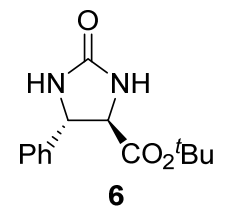

${ }^{13} \mathrm{C} \mathrm{NMR,} \mathrm{CDCl}_{3}, 75 \mathrm{MHz}$
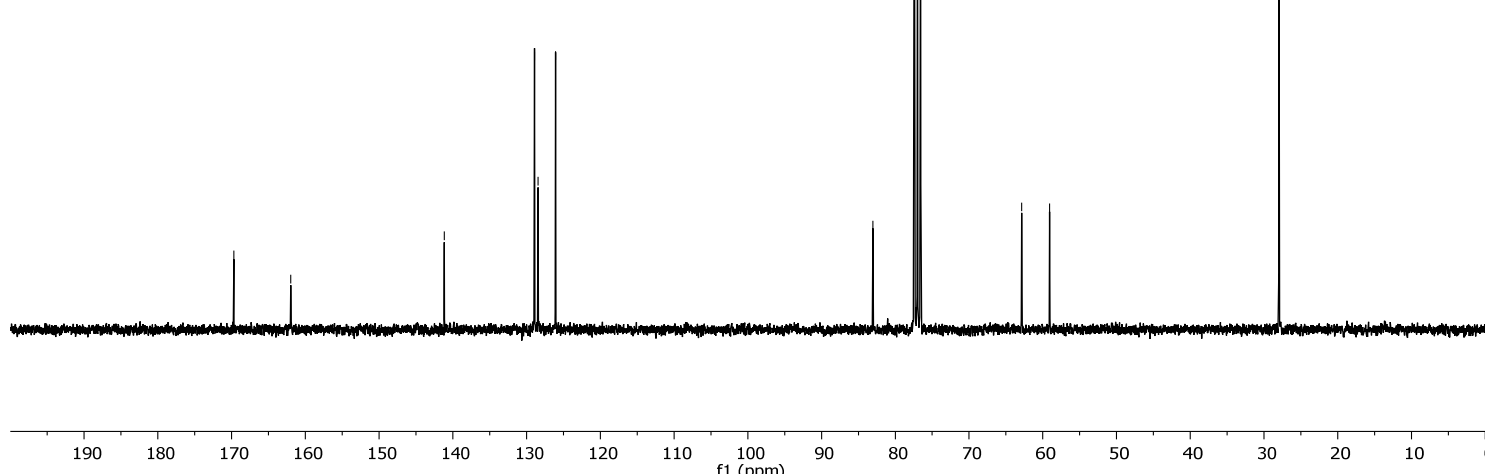

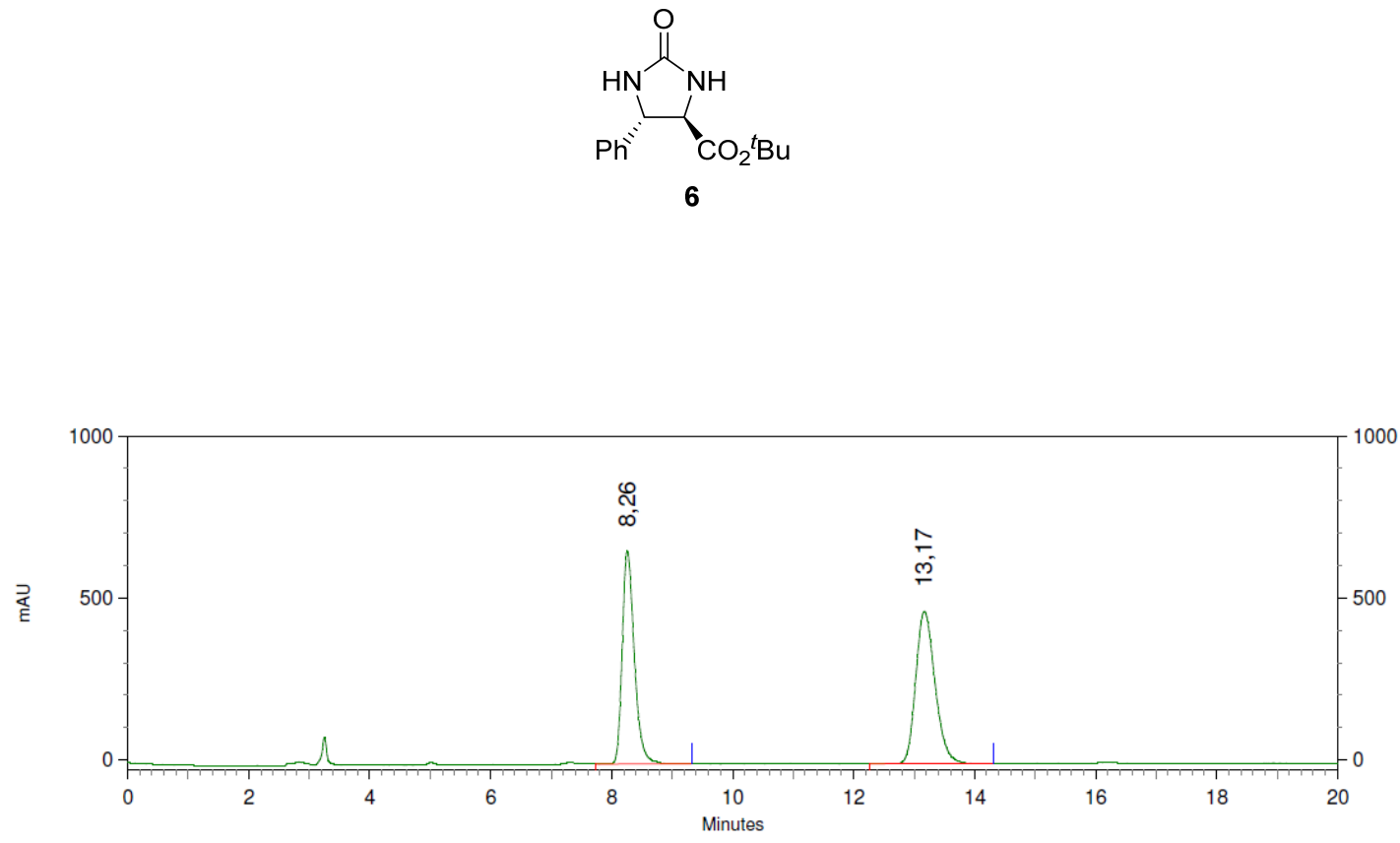

6: $217 \mathrm{~nm}, 4 \mathrm{~nm}$ Results Retention Time

\begin{tabular}{|c|c|c|}
\hline $\begin{array}{r}8,26 \\
13,17\end{array}$ & $\begin{array}{l}36449617 \\
41620989\end{array}$ & $\begin{array}{l}46,688 \\
53,312\end{array}$ \\
\hline
\end{tabular}

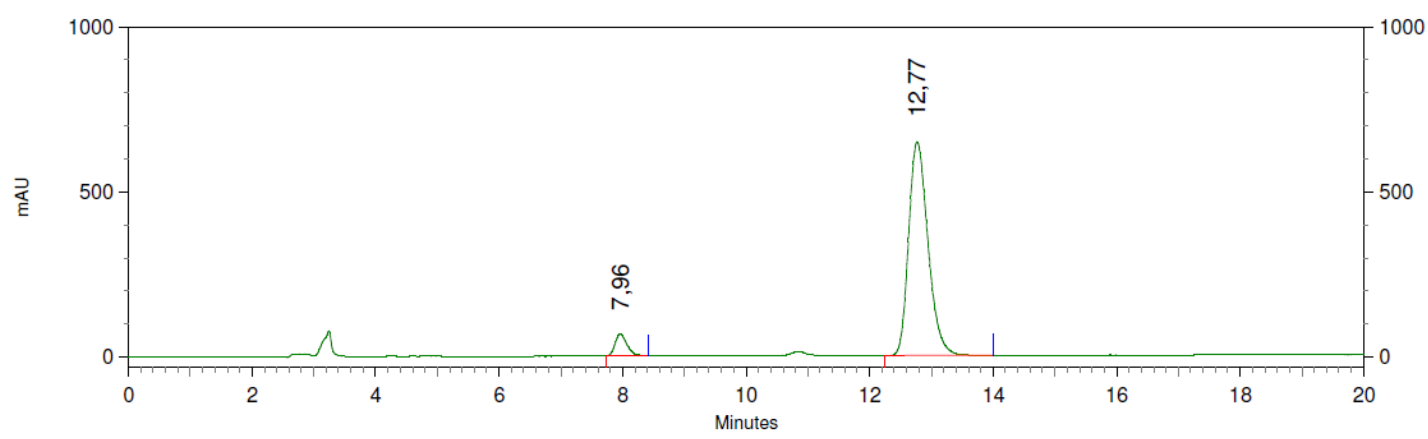

6: 217 nm, 4 nm Results Retention Time Area Area Percent

$\begin{array}{rrr}7,96 & 3491306 & 5,840 \\ 12,77 & 56292313 & 94,160\end{array}$




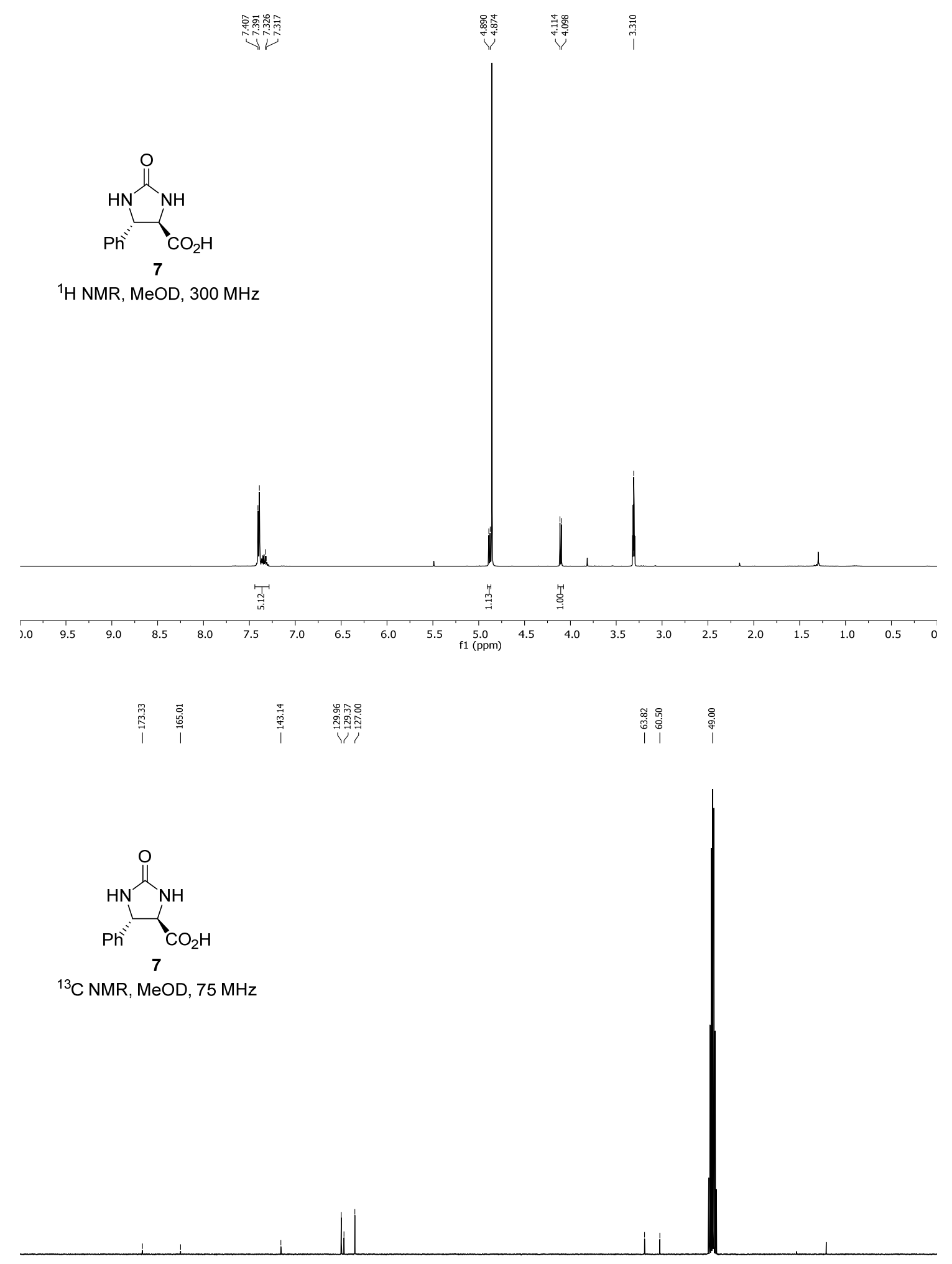

\begin{tabular}{llllllllllllllllllll}
\hline 00 & 190 & 180 & 170 & 160 & 150 & 140 & 130 & 120 & 110 & $\begin{array}{c}100 \\
\mathrm{f} 1(\mathrm{ppm})\end{array}$ & 90 & 80 & 70 & 60 & 50 & 40 & 30 & 20 & 10 \\
\hline
\end{tabular} 


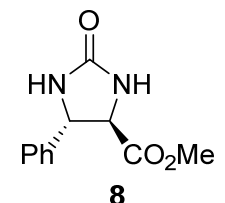

${ }^{1} \mathrm{H}$ NMR, MeOD, $300 \mathrm{MHz}$

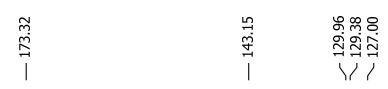

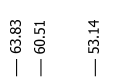

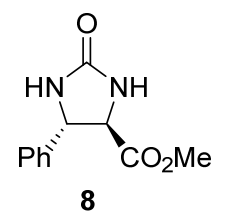

${ }^{13} \mathrm{C}$ NMR, MeOD, $75 \mathrm{MHz}$

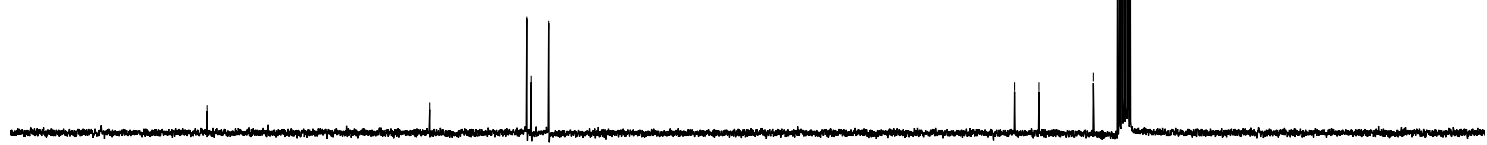

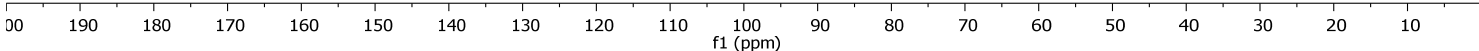



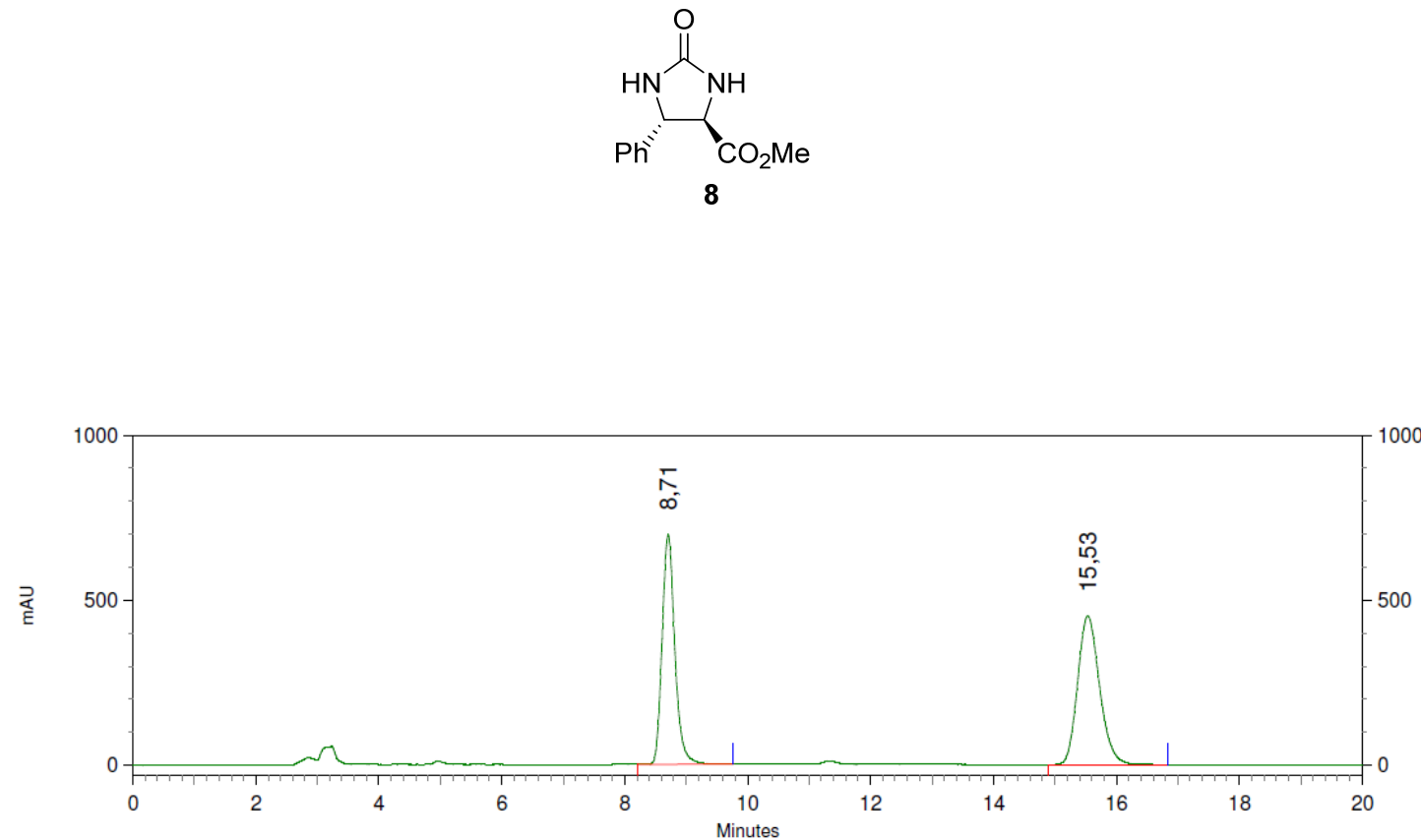

6: $217 \mathrm{~nm}, 4 \mathrm{~nm}$ Results Retention Time

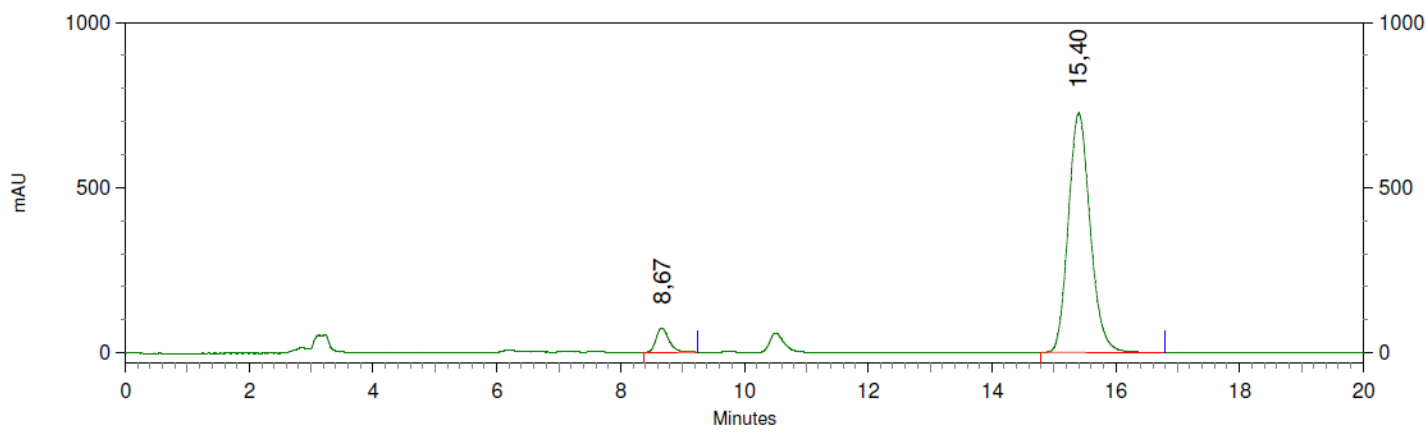

6: $217 \mathrm{~nm}, 4 \mathrm{~nm}$ Results

Retention Time

Area

Area Percent

8,67
15,40 